Supporting Information

for the

Article Entitled

\title{
Impact of Ligands and Metals on the Formation of Metallacyclic Intermediates and a Non-traditional Mechanism for Group VI Alkyne Metathesis Catalysts
}

\author{
Authored by \\ Richard R. Thompson, Madeline E. Rotella, Xin Zhou, Frank R. Fronczek, Osvaldo Gutierrez, \\ Semin Lee \\ Department of Chemistry \\ Louisiana State University, Baton Rouge, Louisiana 70803 \\ Department of Chemistry and Biochemistry \\ University of Maryland, College Park, Maryland 20742
}

\section{TABLE OF CONTENTS}

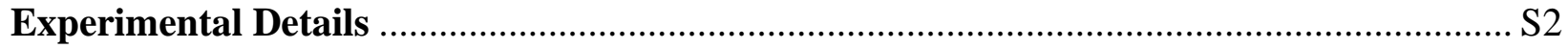

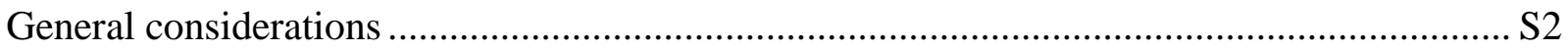

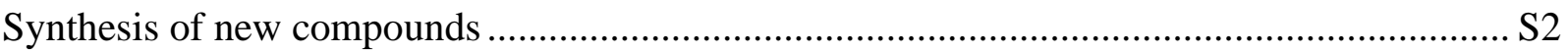

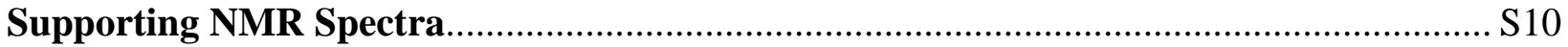

Rate of Alkyne Metathesis using Mo(VI) and W(VI)-based SiP Catalysts ........................ S39

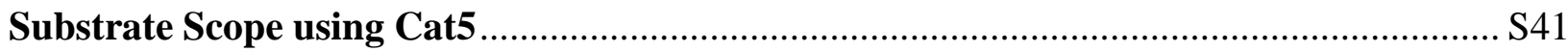

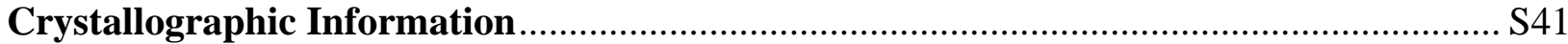

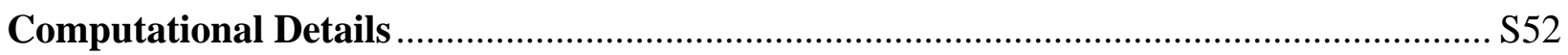

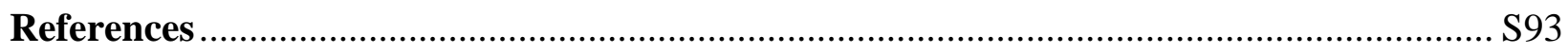




\section{$\underline{\text { Experimental Details }}$}

\section{General considerations}

All manipulations were performed under an inert atmosphere of Ar using standard Schlenkline or glovebox techniques. Anhydrous n-pentane, toluene, diethyl ether and tetrahydrofuran were purchased and dried by passage through two columns of activated alumina and a Q-5 column. Benzene- $d_{6}$ was degassed by three cycles of freeze-pump thaw and then stored over 4 A molecular sieves. Celite and molecular sieves were dried under reduced pressure at $150{ }^{\circ} \mathrm{C}$ for three days. Cooling for the reactions was performed in the internal freezer $\left(-37^{\circ} \mathrm{C}\right)$ of the glovebox used. $\mathrm{Mo}(\mathrm{CO})_{6}, \quad \mathrm{~W}(\mathrm{CO})_{6}, \quad$ 2-bromoacetophenone, oxayl bromide, 1,2-dimethoxyethane, tetramethylammonium bromide, 4-bromotoluene, 2-bromomesitylene, 3-hexyne, 5-decyne, $\mathrm{NaO}{ }^{t} \mathrm{Bu}, \mathrm{Ph}_{2} \mathrm{SiCl}_{2}$ and $\mathrm{Et}_{2} \mathrm{SiCl}_{2}$ were all purchased from Acros Organics and used as received. $\mathrm{C}_{6} \mathrm{H}_{3}\left(\mathrm{C}_{6} \mathrm{H}_{4} \mathrm{Br}\right)_{3},{ }^{1}$ alkynyl substrates, ${ }^{2} \mathrm{Mo}(\equiv \mathrm{CMes}) \mathrm{Br}_{3}(\mathrm{DME}),{ }^{3} \mathrm{~W}(\equiv \mathrm{CMes}) \mathrm{Br}_{3}(\mathrm{DME}),{ }^{3}$ Pre1, ${ }^{4}$ SiPPh5 $^{5}$, Cat1 ${ }^{5}$, and Cat6 $^{5}$ were synthesized via previously reported methods. ${ }^{1} \mathrm{H}$ and ${ }^{13} \mathrm{C}\left\{{ }^{1} \mathrm{H}\right\}$ NMR spectra were recorded on either Varian $400 \mathrm{MHz}$ or $500 \mathrm{MHz}$ NMR spectrometers with benzene- $d_{6}$ referenced at 7.16 and 128.06 ppm, respectively. Low-temperature NMR spectra were collected with dichloromethane- $d_{2}$ referenced at 5.32 and 53.84 , respectively.

\section{Synthesis of new compounds}

\section{$\mathrm{C}_{6} \mathrm{H}_{3}\left(\mathrm{C}_{6} \mathrm{H}_{4} \mathrm{SiEt}_{2} \mathrm{OH}\right)_{3}\left(\mathrm{SiP}^{\mathrm{Et}}\right)$}

To a solution/suspension of $\mathrm{C}_{6} \mathrm{H}_{3}\left(\mathrm{C}_{6} \mathrm{H}_{4} \mathrm{Br}\right)_{3}(1.00 \mathrm{~g}, 1.84$ $\mathrm{mmol})$ in $150 \mathrm{~mL}$ ether was dropwise added $t$-BuLi $(1.8 \mathrm{M}$, $7.2 \mathrm{~mL})$ at $-78{ }^{\circ} \mathrm{C}$. The solution immediately formed precipitates while adding (1-2eq). After further addition (57eq) the precipitates disappeared and formed a transparent yellow-brown solution which gets darker with time. The solution was stirred at $-78{ }^{\circ} \mathrm{C}$ for $4.5 \mathrm{~h}$. $\mathrm{Et}_{2} \mathrm{SiCl}_{2}(0.9 \mathrm{ml}, 6.07$

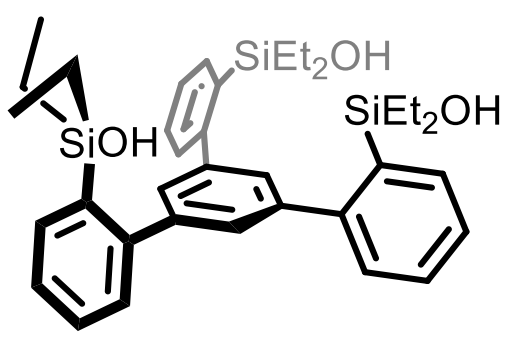
$\mathrm{mmol})$ was added dropwise to the reaction mixture resulting in a red-purple solution. The cooling bath was removed and the reaction mixture was allowed to warm to $\mathrm{rt}$ and stirred overnight. Large amount of salt precipitates form at $\mathrm{rt}$ and the red/brown solution turns to light yellow. The reaction mixture was cooled using an ice bath. The reaction was quenched with water $(\sim 30 \mathrm{~mL})$ and the ice bath was removed. The reaction was stirred at rt for 30 min. The mixture was extracted with ether $(\times 3)$ and washed with brine. The organic phase was collected and dried with $\mathrm{Mg}_{2} \mathrm{SO}_{4}$, then filtered. The solvent was removed under reduced pressure to give a yellow oil. The oil gradually crystallizes over time and samples suitable for X-ray diffraction crystallography were grown by a concentrated pentane solution at $-30{ }^{\circ} \mathrm{C}$. Yield $=$ $0.565 \mathrm{~g}(50 \%) .{ }^{1} \mathrm{H} \mathrm{NMR}\left(400 \mathrm{MHz}, \mathrm{CDCl}_{3}, 25^{\circ} \mathrm{C}\right): 0.76\left(12 \mathrm{H}, \mathrm{q},{ }^{3} \mathrm{~J}_{\mathrm{HH}}=7.6 \mathrm{~Hz}, \mathrm{SiCH}_{2} \mathrm{CH}_{3}\right), 0.87$ $\left(18 \mathrm{H}, \mathrm{t},{ }^{3} \mathrm{~J}_{\mathrm{HH}}=7.6 \mathrm{~Hz}, \mathrm{SiCH}_{2} \mathrm{CH}_{3}\right), 3.60(3 \mathrm{H}, \mathrm{br} \mathrm{s}, \mathrm{SiOH}), 7.23\left(3 \mathrm{H}, \mathrm{s}, \mathrm{C}_{6} H_{3}\right), 7.32\left(3 \mathrm{H}, \mathrm{t},{ }^{3} \mathrm{~J}_{\mathrm{HH}}=\right.$ 7.6 Hz, Ar- H), 7.34-7.41 (6H, m, Ar- H), $7.48\left(3 \mathrm{H}, \mathrm{d},{ }^{3} \mathrm{~J}_{\mathrm{HH}}=7.2 \mathrm{~Hz}, \mathrm{Ar}-H\right) .{ }^{13} \mathrm{C}\left\{{ }^{1} \mathrm{H}\right\} \mathrm{NMR}(125.77$ $\left.\mathrm{MHz}, \mathrm{C}_{6} \mathrm{D}_{6}, 25^{\circ} \mathrm{C}\right): 6.91\left(\mathrm{SiCH}_{2} \mathrm{CH}_{3}\right), 8.28\left(\mathrm{SiCH}_{2} \mathrm{CH}_{3}\right), 126.43(\mathrm{Ar}), 127.72(\mathrm{Ar}), 128.95(\mathrm{Ar})$, $129.94(A r), 134.68(A r), 136.00(A r), 144.20(A r), 149.08(A r)$. HRMS-ESI (m/z): calcd. for $\mathrm{C}_{36} \mathrm{H}_{49} \mathrm{O}_{3} \mathrm{Si}_{3}{ }^{+},[\mathrm{M}+\mathrm{H}]^{+}, 613.2984$, found, 613.3002. 


\section{$\left({ }^{\mathrm{B}} \mathrm{BuO}\right)_{3} \mathrm{~W} \equiv$ CMes $($ Pre2)}

$\mathrm{W}(\equiv \mathrm{CMes}) \mathrm{Br}_{3}(\mathrm{DME})(495 \mathrm{mg} / 0.75 \mathrm{mmol})$ was dissolved into $10 \mathrm{ml}$ of THF and $\mathrm{NaO}^{\mathrm{t}} \mathrm{Bu}(229 \mathrm{mg} / 2.38 \mathrm{mmol})$ was dissolved in $5 \mathrm{ml}$ of THF and both solutions were chilled to $-37^{\circ} \mathrm{C}$. The colorless solution of $\mathrm{NaO}^{t} \mathrm{Bu}$ was dropwise added to the stirring, green solution of $\mathrm{W}(\equiv \mathrm{CMes}) \mathrm{Br}_{3}(\mathrm{DME})$, resulting in the solution becoming yellow-brown and the precipitation of $\mathrm{KBr}$ by-product. The reaction stirred at roomtemperature for $0.5 \mathrm{~h}$ at which point solvent was removed under reduced pressure, the product extracted into $10 \mathrm{ml}$ of pentane and the $\mathrm{KBr}$ impurity removed by filtering through 3" celite plug on a medium porosity fritted funnel. Removal of all volatile resulted in material pure enough for subsequent chemistry, storage of a concentrated pentane

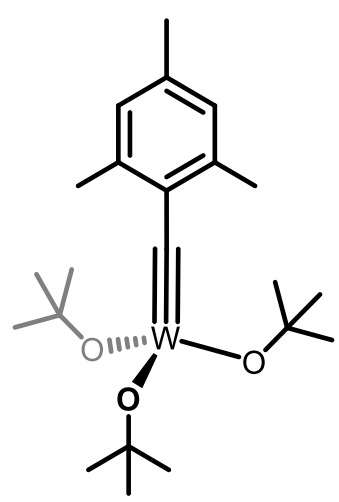
solution at $-37{ }^{\circ} \mathrm{C}$ resulted in single crystals suitable for X-ray diffraction studies. Yield (of crystalline material $)=204 \mathrm{mg}(51.0 \%) .{ }^{1} \mathrm{H} \mathrm{NMR}\left(500 \mathrm{MHz}, \mathrm{C}_{6} \mathrm{D}_{6}, 25{ }^{\circ} \mathrm{C}\right): 1.43\left(27 \mathrm{H}, \mathrm{s}, \mathrm{C}\left(\mathrm{CH}_{3}\right)_{3}\right)$, $2.27\left(3 \mathrm{H}, \mathrm{s}, p-\mathrm{CH}_{3}\right), 2.93\left(6 \mathrm{H}, \mathrm{s}, o-\mathrm{CH}_{3}\right), 6.87\left(2 \mathrm{H}, \mathrm{s}, o-\mathrm{C}_{6} \mathrm{H}_{2} \mathrm{Me}_{3}\right) .{ }^{13} \mathrm{C}\left\{{ }^{1} \mathrm{H}\right\} \mathrm{NMR}(125.77 \mathrm{MHz}$, $\left.\mathrm{C}_{6} \mathrm{D}_{6}, 25^{\circ} \mathrm{C}\right): 271.33(\mathrm{~W} \equiv C), 143.2(A r), 140.6(A r), 135.1(A r), 127.6(A r), 79.6\left(C\left(\mathrm{CH}_{3}\right)_{3}\right), 32.2$ $\left(\mathrm{C}\left(\mathrm{CH}_{3}\right)_{3}\right), 21.7\left(o-\mathrm{CH}_{3}\right), 20.9\left(p-\mathrm{CH}_{3}\right)$.

\section{$\left[\mathrm{C}_{6} \mathrm{H}_{3}\left(\mathrm{C}_{6} \mathrm{H}_{4} \mathrm{SiPh}_{2} \mathrm{O}\right)_{3}\right] \mathrm{Mo} \equiv \mathrm{CMes}(\mathrm{Cat} 2)$}

$\mathrm{Mo}(\equiv \mathrm{CMes}) \mathrm{Br}_{3}(\mathrm{DME})(1.00 \mathrm{~g} / 1.80 \mathrm{mmol})$ was dissolved into $10 \mathrm{ml}$ of $\mathrm{THF}$ and $\mathrm{NaO}^{\mathrm{t}} \mathrm{Bu}(0.518 \mathrm{~g} / 5.39 \mathrm{mmol})$ was dissolved in $10 \mathrm{ml}$ of THF and both solutions were chilled to $-37^{\circ} \mathrm{C}$. The colorless solution of $\mathrm{NaO}^{\mathrm{t}} \mathrm{Bu}$ was dropwise added to the stirring, green solution of $\mathrm{Mo}(\equiv \mathrm{CMes}) \mathrm{Br}_{3}(\mathrm{DME})$, resulting in the solution becoming yellowbrown and the precipitation of $\mathrm{KBr}$ by-product. The reaction stirred at room-temperature for $0.5 \mathrm{~h}$ at which point solvent was removed under reduced pressure, the product extracted into $10 \mathrm{ml}$ of pentane and the $\mathrm{KBr}$ impurity removed by filtering through 3" celite plug on a medium

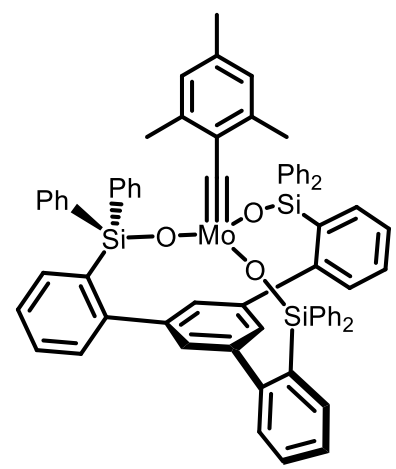
porosity fritted funnel. The volatiles were removed under reduced pressure and the resulting offwhite solids were redissolved into $10 \mathrm{ml}$ toluene and $\mathbf{S i P} \mathbf{P}^{\mathbf{P h}}(1.62 \mathrm{~g} / 1.80 \mathrm{mmol})$ was added directly to the solution, resulting in a color change from yellow to orange. After stirring for $10 \mathrm{~min}$. the volatiles were removed under reduced pressure. Single crystals (orange plates) suitable for X-ray diffraction studies were grown from slow-evaporation of dichloromethane orange, pure powder was obtained by chilling a concentrated toluene solution to $-37{ }^{\circ} \mathrm{C}$. Yield $=1.64 \mathrm{~g}(81.0 \%) .{ }^{1} \mathrm{H}$ NMR (500 MHz, $\left.\mathrm{C}_{6} \mathrm{D}_{6}, 25^{\circ} \mathrm{C}\right): 2.14\left(6 \mathrm{H}, \mathrm{s}, o-\mathrm{CH}_{3}\right), 2.16\left(3 \mathrm{H}, \mathrm{s}, p-\mathrm{CH}_{3}\right), 6.48\left(2 \mathrm{H}, \mathrm{s}, \mathrm{C}_{6} \mathrm{H}_{2} \mathrm{Me}_{3}\right)$, $6.90\left(3 \mathrm{H}, \mathrm{d},{ }^{3} \mathrm{~J}_{\mathrm{HH}}=10.0 \mathrm{~Hz}, \mathrm{Ar}-H\right), 7.03-7.10(24 \mathrm{H}, \mathrm{m}$, Overlap of several Ar- $H), 7.28(3 \mathrm{H}, \mathrm{s}$, $\left.\mathrm{C}_{6} \mathrm{H}_{3} \mathrm{Ar}_{3}\right), 7.76\left(3 \mathrm{H}, \mathrm{d},{ }^{3} \mathrm{~J}_{\mathrm{HH}}=10.0 \mathrm{~Hz}, \mathrm{Ar}-H\right), 7.83\left(12 \mathrm{H}, \mathrm{d},{ }^{3} \mathrm{~J}_{\mathrm{HH}}=10.0 \mathrm{~Hz}, o-\mathrm{SiPh}_{2}\right) .{ }^{13} \mathrm{C}\left\{{ }^{1} \mathrm{H}\right\}$ NMR (125.77 MHz, $\left.\mathrm{C}_{6} \mathrm{D}_{6}, 25^{\circ} \mathrm{C}\right): 288.42(\mathrm{~W} \equiv C), 149.50(A r), 144.31(A r), 141.77(A r), 137.59$ (Ar), $137.28(A r), 136.01(A r), 135.12(A r), 130.47$ (Ar), $130.24(A r), 130.00(A r), 129.13(A r)$, 128.59 (Ar), 128.48 (Ar), 128.35 (Ar), 126.35 (Ar), $125.93(A r), 20.54\left(p-C \mathrm{H}_{3}\right), 20.13\left(o-C \mathrm{H}_{3}\right)$. 


\section{$\left[\mathrm{C}_{6} \mathrm{H}_{3}\left(\mathrm{C}_{6} \mathrm{H}_{4} \mathrm{SiPh}_{2} \mathrm{O}\right)_{3}\right] \mathrm{W} \equiv \mathrm{CMes}(\mathrm{Cat} 3)$}

$\mathrm{W}$ ( $\equiv \mathrm{CMes}) \mathrm{Br}_{3}$ (DME) $(1.00 \mathrm{~g} / 1.52 \mathrm{mmol})$ was dissolved into $10 \mathrm{ml}$ of THF and $\mathrm{NaO}^{\mathrm{t}} \mathrm{Bu}(0.437 \mathrm{mg} / 4.54 \mathrm{mmol})$ was dissolved in $10 \mathrm{ml}$ of THF and both solutions were chilled to $-37^{\circ} \mathrm{C}$. The colorless solution of $\mathrm{NaO}^{t} \mathrm{Bu}$ was dropwise added to the stirring, green solution of $\mathrm{W}(\equiv \mathrm{CMes}) \mathrm{Br}_{3}(\mathrm{DME})$, resulting in the solution becoming yellow-brown and the precipitation of $\mathrm{KBr}$ by-product. The reaction stirred at room-temperature for $0.5 \mathrm{~h}$ at which point solvent was removed under reduced pressure, the product extracted into $10 \mathrm{ml}$ of pentane and the $\mathrm{KBr}$ impurity removed by filtering through 3" celite plug on a medium porosity fritted funnel. The volatiles were removed under reduced pressure and the resulting offwhite solids were redissolved into $10 \mathrm{ml}$ toluene and $\mathbf{S i P}^{\mathbf{P h}}(1.37$

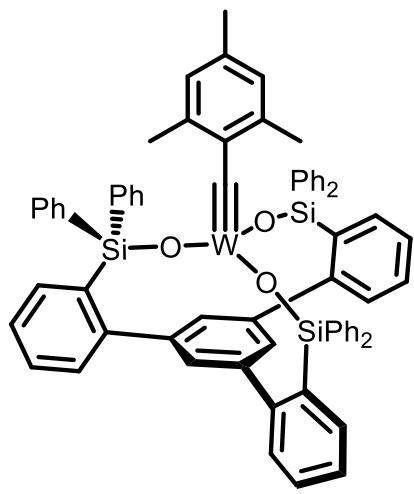
$\mathrm{g} / 1.52 \mathrm{mmol}$ ) was added directly to the solution, resulting in a color change from yellow to orange. After stirring for $10 \mathrm{~min}$. the volatiles were removed under reduced pressure. Single crystals (orange plates) suitable for X-ray diffraction studies were grown from slow-evaporation of dichloromethane orange, pure powder was obtained by chilling a concentrated toluene solution to $-37{ }^{\circ} \mathrm{C}$. Yield $=1.47 \mathrm{~g}(79.6 \%) .{ }^{1} \mathrm{H}$ NMR $\left(500 \mathrm{MHz}, \mathrm{C}_{6} \mathrm{D}_{6}, 25^{\circ} \mathrm{C}\right): 2.14\left(6 \mathrm{H}, \mathrm{s}, o-\mathrm{CH}_{3}\right), 2.16(3 \mathrm{H}$, $\left.\mathrm{s}, p-\mathrm{CH}_{3}\right), 6.48\left(2 \mathrm{H}, \mathrm{s}, \mathrm{C}_{6} \mathrm{H}_{2} \mathrm{Me}_{3}\right), 6.90\left(3 \mathrm{H}, \mathrm{d},{ }^{3} \mathrm{~J}_{\mathrm{HH}}=10.0 \mathrm{~Hz}, \mathrm{Ar}-H\right), 7.03-7.10(24 \mathrm{H}, \mathrm{m}$, Overlap of several Ar-H), $7.28\left(3 \mathrm{H}, \mathrm{s}, \mathrm{C}_{6} \mathrm{H}_{3} \mathrm{Ar}_{3}\right), 7.76\left(3 \mathrm{H}, \mathrm{d},{ }^{3} \mathrm{~J}_{\mathrm{HH}}=10.0 \mathrm{~Hz}, \mathrm{Ar}-H\right), 7.83\left(12 \mathrm{H}, \mathrm{d},{ }^{3} \mathrm{~J}_{\mathrm{HH}}=\right.$ $\left.10.0 \mathrm{~Hz}, o-\mathrm{SiPh}_{2}\right) .{ }^{13} \mathrm{C}\left\{{ }^{1} \mathrm{H}\right\} \mathrm{NMR}\left(125.77 \mathrm{MHz}, \mathrm{C}_{6} \mathrm{D}_{6}, 25^{\circ} \mathrm{C}\right): 288.42(\mathrm{~W} \equiv C), 149.50(A r), 144.31$ (Ar), $141.77(A r), 137.59(A r), 137.28(A r), 136.01(A r), 135.12(A r), 130.47(A r), 130.24(A r)$, 130.00 (Ar), 129.13 (Ar), 128.59 (Ar), 128.48 (Ar), 128.35 (Ar), 126.35 (Ar), 125.93 (Ar), 20.54 $\left(p-\mathrm{CH}_{3}\right), 20.13\left(o-\mathrm{CH}_{3}\right)$.

\section{$\left[\mathrm{C}_{6} \mathrm{H}_{3}\left(\mathrm{C}_{6} \mathrm{H}_{4} \mathrm{SiEt}_{2} \mathrm{O}\right)_{3}\right] \mathrm{Mo} \equiv \mathrm{CMes}(\mathrm{Cat} 4)$}

$\mathrm{Mo}$ (三CMes)Br $\mathrm{Br}_{3}(\mathrm{DME})(280 \mathrm{mg} / 0.53 \mathrm{mmol})$ was dissolved into 10 $\mathrm{ml}$ of THF and $\mathrm{NaO}^{\mathrm{t}} \mathrm{Bu}(158 \mathrm{mg} / 1.59 \mathrm{mmol})$ was dissolved in $10 \mathrm{ml}$ of THF and both solutions were chilled to $-37{ }^{\circ} \mathrm{C}$. The colorless solution of $\mathrm{NaO}^{\mathrm{t}} \mathrm{Bu}$ was dropwise added to the stirring, brown solution of $\mathrm{Mo}(\equiv \mathrm{CMes}) \mathrm{Br}_{3}(\mathrm{DME})$, resulting in the solution becoming yellow-brown and the precipitation of $\mathrm{KBr}$ by-product. The reaction stirred at room-temperature for $0.5 \mathrm{~h}$ at which point solvent was removed under reduced pressure, the product extracted into $10 \mathrm{ml}$ of pentane and the $\mathrm{KBr}$ impurity removed by filtering through 3" celite plug on a medium porosity fritted funnel. The volatiles were removed under reduced pressure and the resulting offwhite solids were redissolved into $10 \mathrm{ml}$ pentane and $\mathbf{S i P}^{\mathbf{E t}}$ (308 $\mathrm{g} / 0.53 \mathrm{mmol}$ ) was added directly to the solution, resulting in a color

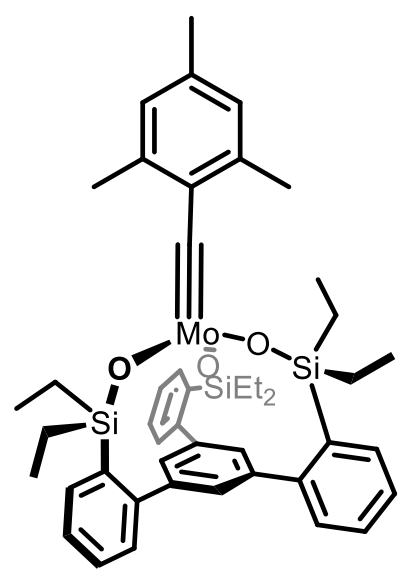
change from yellow to orange-yellow. After stirring for $10 \mathrm{~min}$. the volatiles were removed under reduced pressure. Single crystals (yellow plates) suitable for X-ray diffraction studies were grown from a concentrated $\mathrm{Et}_{2} \mathrm{O}$ solution stored at $-37{ }^{\circ} \mathrm{C}$. Yield $=337 \mathrm{mg}(75.5 \%)$. ${ }^{1} \mathrm{H}$ NMR $(400 \mathrm{MHz}$, $\left.\mathrm{C}_{6} \mathrm{D}_{6}, 25^{\circ} \mathrm{C}\right): 0.96\left(12 \mathrm{H}, \mathrm{q},{ }^{3} \mathrm{~J}_{\mathrm{HH}}=4.0 \mathrm{~Hz}, \mathrm{SiCH}_{2} \mathrm{CH}_{3}\right), 1.01\left(18 \mathrm{H}, \mathrm{t},{ }^{3} \mathrm{~J}_{\mathrm{HH}}=4.0 \mathrm{~Hz}, \mathrm{SiCH}_{2} \mathrm{CH}_{3}\right)$, $2.09\left(3 \mathrm{H}, \mathrm{s}, p-\mathrm{CH}_{3}\right), 2.72\left(6 \mathrm{H}, \mathrm{s}, p-\mathrm{CH}_{3}\right), 6.63\left(2 \mathrm{H}, \mathrm{s}, \mathrm{C}_{6} \mathrm{H}_{2} \mathrm{Me}_{3}\right), 7.17-7.19(12 \mathrm{H}, \mathrm{m}, \mathrm{Ar}-\mathrm{H}), 7.28$ $\left(3 \mathrm{H}, \mathrm{d},{ }^{3} \mathrm{~J}_{\mathrm{HH}}=8.0 \mathrm{~Hz}, \mathrm{Ar}-H\right), 7.45\left(3 \mathrm{H}, \mathrm{d},{ }^{3} \mathrm{~J}_{\mathrm{HH}}=8.0 \mathrm{~Hz}, \mathrm{Ar}-H\right), 7.50\left(3 \mathrm{H}, \mathrm{s}, \mathrm{C}_{6} H_{3}\right) .{ }^{13} \mathrm{C}\left\{{ }^{1} \mathrm{H}\right\} \mathrm{NMR}$ 
(125.77 MHz, $\left.\mathrm{C}_{6} \mathrm{D}_{6}, 25^{\circ} \mathrm{C}\right): 306.7(\mathrm{Mo} \equiv C), 149.68(A r), 144.69(A r), 143.73(A r), 138.38(A r)$, 137.19 (Ar), 136.49 (Ar), 134.80 (Ar), 130.81 (Ar), 129.13 (Ar), 138.58 (Ar), 127.51 (Ar), 126.72 (Ar), $21.07\left(o-\mathrm{CH}_{3}\right), 20.78\left(p-\mathrm{CH}_{3}\right), 9.62\left(\mathrm{SiCH}_{2} \mathrm{CH}_{3}\right), 7.31\left(\mathrm{SiCH}_{2} \mathrm{CH}_{3}\right)$.

\section{$\left[\mathrm{C}_{6} \mathrm{H}_{3}\left(\mathrm{C}_{6} \mathrm{H}_{4} \mathrm{SiEt}_{2} \mathrm{O}\right)_{3}\right] \mathrm{W} \equiv \mathrm{CMes}(\mathrm{Cat5})$}

$\mathrm{W}(\equiv \mathrm{CMes}) \mathrm{Br}_{3}(\mathrm{DME})(250 \mathrm{mg} / 0.38 \mathrm{mmol})$ was dissolved into $10 \mathrm{ml}$ of $\mathrm{THF}$ and $\mathrm{NaO}^{t} \mathrm{Bu}(125 \mathrm{mg} / 1.14 \mathrm{mmol})$ was dissolved in $10 \mathrm{ml}$ of THF and both solutions were chilled to $-37^{\circ} \mathrm{C}$. The colorless solution of $\mathrm{NaO}^{t} \mathrm{Bu}$ was dropwise added to the stirring, green solution of $\mathrm{W}(\equiv \mathrm{CMes}) \mathrm{Br}_{3}(\mathrm{DME})$, resulting in the solution becoming yellowbrown and the precipitation of $\mathrm{KBr}$ by-product. The reaction stirred at room-temperature for $0.5 \mathrm{~h}$ at which point solvent was removed under reduced pressure, the product extracted into $10 \mathrm{ml}$ of pentane and the $\mathrm{KBr}$ impurity removed by filtering through 3" celite plug on a medium porosity fritted funnel. The volatiles were removed under reduced pressure and the resulting off-white solids were redissolved into $10 \mathrm{ml}$ pentane and $\mathbf{S i P}^{\mathbf{E t}}(238 \mathrm{mg} / 0.38 \mathrm{mmol})$ was added directly to the solution. After stirring for $10 \mathrm{~min}$. the volatiles were removed

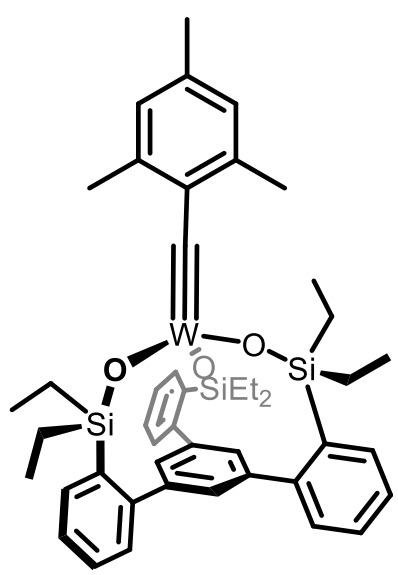
under reduced pressure. Single crystals (yellow plates) suitable for X-ray diffraction studies were grown from a concentrated pentane solution to $-37{ }^{\circ} \mathrm{C}$. Yield $=260 \mathrm{mg}(73.7 \%) .{ }^{1} \mathrm{H}$ NMR $(400$ $\left.\mathrm{MHz}, \mathrm{C}_{6} \mathrm{D}_{6}, 25{ }^{\circ} \mathrm{C}\right): 0.86-0.97\left(12 \mathrm{H}, \mathrm{m}, \mathrm{SiCH}_{2} \mathrm{CH}_{3}\right), 1.00\left(18 \mathrm{H}, \mathrm{m}, \mathrm{SiCH}_{2} \mathrm{CH}_{3}\right), 2.33$ (3H, s, $p$ $\left.\mathrm{CH}_{3}\right), 2.89\left(6 \mathrm{H}, \mathrm{s}, o-\mathrm{CH}_{3}\right), 6.88\left(2 \mathrm{H}, \mathrm{s}, \mathrm{C}_{6} \mathrm{H}_{2} \mathrm{Me}_{3}\right), 7.18(3 \mathrm{H}, \mathrm{m}, \mathrm{Ar}-H), 7.26\left(3 \mathrm{H}, \mathrm{t},{ }^{3} \mathrm{~J}_{\mathrm{HH}}=8.4 \mathrm{~Hz}\right.$, $\operatorname{Ar}-H), 7.39\left(3 \mathrm{H}, \mathrm{dd},{ }^{3} \mathrm{~J}_{\mathrm{HH}}=8.4 \mathrm{~Hz}, \mathrm{Ar}-H\right), 7.47\left(3 \mathrm{H}, \mathrm{s}, \mathrm{C}_{6} H_{3}\right) .{ }^{13} \mathrm{C}\left\{{ }^{1} \mathrm{H}\right\} \mathrm{NMR}\left(125.77 \mathrm{MHz}, \mathrm{C}_{6} \mathrm{D}_{6}\right.$, $\left.25{ }^{\circ} \mathrm{C}\right): 281.9(\mathrm{~W} \equiv C), 149.5(A r), 144.7(A r), 142.6(A r), 140.6(A r), 135.9(A r), 135.8(A r), 134.7$ (Ar), 130.8 (Ar), 126.8 (Ar), 126.7 (Ar), $20.9\left(o-\mathrm{CH}_{3}\right), 20.7\left(p-\mathrm{CH}_{3}\right), 9.3\left(\mathrm{SiCH}_{2} \mathrm{CH}_{3}\right), 7.1$ $\left(\mathrm{SiCH}_{2} \mathrm{CH}_{3}\right)$.

\section{$\left(\mathrm{Ph}_{3} \mathrm{SiO}\right)_{3} \mathrm{Mo}\left(\mathrm{C}_{3} \mathrm{Et}_{3}\right)(\mathrm{MCBD1})$}

Cat6 $(60 \mathrm{mg} / 0.059 \mathrm{mmol})$ was dissolved into $0.6 \mathrm{ml}$ of $\mathrm{CD}_{2} \mathrm{Cl}_{2}$ and 3hexyne $(29 \mathrm{mg} / 0.351 \mathrm{mmol})$ was added. The solution was cooled to $-70{ }^{\circ} \mathrm{C}$ and subjected to NMR interrogation. ${ }^{1} \mathrm{H}$ NMR $\left(500 \mathrm{MHz}, \mathrm{CD}_{2} \mathrm{Cl}_{2},-70{ }^{\circ} \mathrm{C}\right)$ : $0.03\left(3 \mathrm{H}, \mathrm{t},{ }^{3} \mathrm{~J}_{\mathrm{HH}}=8.0 \mathrm{~Hz}, \beta-\mathrm{CCH}_{2} \mathrm{CH}_{3}\right), 1.49\left(6 \mathrm{H}, \mathrm{t},{ }^{3} \mathrm{~J}_{\mathrm{HH}}=8.0 \mathrm{~Hz}, \alpha-\right.$ $\left.\mathrm{CCH}_{2} \mathrm{CH}_{3}\right), 2.87\left(4 \mathrm{H}, \mathrm{q},{ }^{3} \mathrm{~J}_{\mathrm{HH}}=7.5 \mathrm{~Hz}, \alpha-\mathrm{CCH}_{2} \mathrm{CH}_{3}\right), 2.14\left(2 \mathrm{H}, \mathrm{q},{ }^{3} \mathrm{~J}_{\mathrm{HH}}=\right.$ $\left.8.0 \mathrm{~Hz}, \beta-\mathrm{CCH}_{2} \mathrm{CH}_{3}\right), 2.87\left(4 \mathrm{H}, \mathrm{q},{ }^{3} \mathrm{~J}_{\mathrm{HH}}=8.0 \mathrm{~Hz}, \alpha-\mathrm{CCH}_{2} \mathrm{CH}_{3}\right), 7.00(6 \mathrm{H}, \mathrm{t}$, $\left.{ }^{3} \mathrm{~J}_{\mathrm{HH}}=8.0 \mathrm{~Hz}, p-\mathrm{C}_{6} H_{5}\right), 7.10\left(12 \mathrm{H}, \mathrm{t},{ }^{3} \mathrm{~J}_{\mathrm{HH}}=8.0 \mathrm{~Hz}, p-\mathrm{C}_{6} H_{5}\right) 7.15-7.30(18 \mathrm{H}$, $\left.\mathrm{m}, m-\mathrm{C}_{6} H_{5}\right), 7.38\left(12 \mathrm{H}, \mathrm{d},{ }^{3} \mathrm{~J}_{\mathrm{HH}}=8.0 \mathrm{~Hz}, o-\mathrm{C}_{6} H_{5}\right), 7.78\left(6 \mathrm{H}, \mathrm{d},{ }^{3} \mathrm{~J}_{\mathrm{HH}}=4.0\right.$

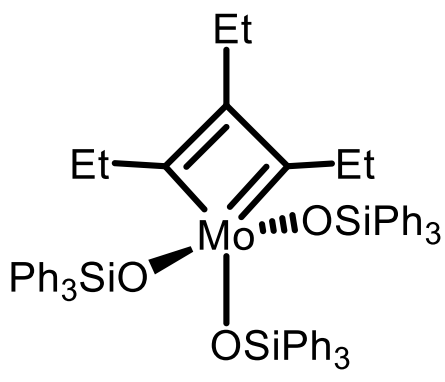
$\left.\mathrm{Hz}, o-\mathrm{C}_{6} \mathrm{H}_{5}\right) .{ }^{13} \mathrm{C}\left\{{ }^{1} \mathrm{H}\right\}$ NMR $\left(125 \mathrm{MHz}, \mathrm{CD}_{2} \mathrm{Cl}_{2},-70{ }^{\circ} \mathrm{C}\right): 249.78\left(\alpha-\mathrm{CCH}_{2} \mathrm{CH}_{3}\right), 147.15(\beta-$ $\mathrm{CCH}_{2} \mathrm{CH}_{3}$ ), 147.15 (Ar), 137.88 (Ar), 137.83 (Ar), 137.27 (Ar), 135.44 (Ar), 134.87 (Ar), 134.81 (Ar), 134.58 (Ar), $131.17(A r), 131.11(A r), 130.07$ (Ar), 129.27 (Ar), $129.21(A r), 129.03(A r)$, 128.95 (Ar), $128.78(A r), 128.16(A r), 127.83(A r), 127.71(A r), 127.41(A r), 125.21(A r), 120.25$ $(\mathrm{Ar}), 31.56\left(\alpha-\mathrm{CCH}_{2} \mathrm{CH}_{3}\right), 26.06\left(\beta-\mathrm{CCH}_{2} \mathrm{CH}_{3}\right), 13.84\left(\alpha-\mathrm{CCH}_{2} \mathrm{CH}_{3}\right), 11.22\left(\beta-\mathrm{CCH}_{2} \mathrm{CH}_{3}\right)$.

\section{$\left[\mathrm{C}_{6} \mathrm{H}_{3}\left(\mathrm{C}_{6} \mathrm{H}_{4} \mathrm{SiPh}_{2} \mathrm{O}\right)_{3}\right] \mathrm{Mo} \equiv \mathrm{CBu}(\mathrm{Cat} 7)$}

Cat1 $(375 \mathrm{mg} / 0.320 \mathrm{mmol})$ was dissolved into $10 \mathrm{ml}$ of toluene and 5-decyne $(131 \mathrm{mg} / 0.960$ mmol) leading to a dark brown solution. The solution was allowed to stir for $0.5 \mathrm{~h}$ at roomtemperature. All volatiles were removed under reduced pressure and the crude product was washed 
with pentane to give a grey-brown solid. The crude solid was redissolved in toluene and stirred over $5 \AA$ A molecular sieves $(500 \mathrm{mg})$ for $2 \mathrm{~h}$. The sieves were removed via filtration through a fine porosity fritted funnel and the volatiles of the supernatant were removed under reduced pressure while heating to $50{ }^{\circ} \mathrm{C}$ to give a yellow solid. Yield $=78.6 \mathrm{mg}(23.1 \%) .{ }^{1} \mathrm{H}$ NMR $\left(500 \mathrm{MHz}, \mathrm{C}_{6} \mathrm{D}_{6}, 25{ }^{\circ} \mathrm{C}\right): 0.39(3 \mathrm{H}, \mathrm{t}$, $\left.{ }^{3} \mathrm{~J}_{\mathrm{HH}}=6.0 \mathrm{~Hz}, \mathrm{Mo} \equiv \mathrm{CCH}_{2} \mathrm{CH}_{2} \mathrm{CH}_{2} \mathrm{CH}_{3}\right), 0.39\left(3 \mathrm{H}, \mathrm{t},{ }^{3} \mathrm{~J}_{\mathrm{HH}}=6.0 \mathrm{~Hz}\right.$, $\left.\mathrm{Mo} \equiv \mathrm{CCH}_{2} \mathrm{CH}_{2} \mathrm{CH}_{2} \mathrm{CH}_{3}\right), \quad 0.72 \quad\left(2 \mathrm{H}, \quad \mathrm{m}, \quad{ }^{3} \mathrm{~J}_{\mathrm{HH}}=4.0 \mathrm{~Hz}\right.$, $\mathrm{Mo} \equiv \mathrm{CCH}_{2} \mathrm{CH}_{2} \mathrm{CH}_{2} \mathrm{CH}_{3}, \quad 0.91 \quad\left(2 \mathrm{H}, \quad \mathrm{m}, \quad{ }^{3} \mathrm{~J}_{\mathrm{HH}}=4.0 \mathrm{~Hz}\right.$,

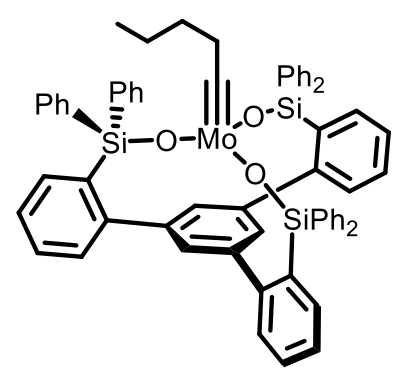
$\left.\mathrm{Mo} \equiv \mathrm{CCH}_{2} \mathrm{CH}_{2} \mathrm{CH}_{2} \mathrm{CH}_{3}\right), 2.56\left(2 \mathrm{H}, \mathrm{t},{ }^{3} \mathrm{~J}_{\mathrm{HH}}=8.0 \mathrm{~Hz}, \mathrm{Mo} \equiv \mathrm{CCH}_{2} \mathrm{CH}_{2} \mathrm{CH}_{2} \mathrm{CH}_{3}\right), 6.87\left(3 \mathrm{H}, \mathrm{d},{ }^{3} \mathrm{~J}_{\mathrm{HH}}=\right.$ 8.0, Ar- $H), 7.05-7.14(24 \mathrm{H}, \mathrm{m}$, Overlap of several $\mathrm{Ar}-\mathrm{H}), 7.24\left(3 \mathrm{H}, \mathrm{s}, \mathrm{C}_{6} \mathrm{H}_{3} \mathrm{Ar}_{3}\right), 7.76\left(3 \mathrm{H}, \mathrm{d},{ }^{3} \mathrm{~J}_{\mathrm{HH}}\right.$ $=8.0 \mathrm{~Hz}, \operatorname{Ar}-H), 7.87\left(12 \mathrm{H}, \mathrm{d},{ }^{3} \mathrm{~J}_{\mathrm{HH}}=8.0 \mathrm{~Hz}, o-\mathrm{SiPh}_{2}\right) .{ }^{13} \mathrm{C}\left\{{ }^{1} \mathrm{H}\right\} \mathrm{NMR}\left(125.77 \mathrm{MHz}, \mathrm{C}_{6} \mathrm{D}_{6}, 25\right.$ $\left.{ }^{\circ} \mathrm{C}\right): 324.87(\mathrm{Mo} \equiv C), 149.53(A r), 144.13(A r), 138.35(A r), 135.10(A r), 130.44(A r), 130.08(A r)$, $129.81 \quad(A r), \quad 129.18 \quad(A r), \quad 126.24 \quad(A r), \quad 50.95 \quad\left(\mathrm{Mo} \equiv \mathrm{CCH}_{2} \mathrm{CH}_{2} \mathrm{CH}_{2} \mathrm{CH}_{3}\right), \quad 31.27$ $\left(\mathrm{Mo} \equiv \mathrm{CCH}_{2} \mathrm{CH}_{2} \mathrm{CH}_{2} \mathrm{CH}_{3}\right), 22.36\left(\mathrm{Mo} \equiv \mathrm{CCH}_{2} \mathrm{CH}_{2} \mathrm{CH}_{2} \mathrm{CH}_{3}\right), 13.59\left(\mathrm{Mo} \equiv \mathrm{CCH}_{2} \mathrm{CH}_{2} \mathrm{CH}_{2} \mathrm{CH}_{3}\right)$.

$\left[\mathrm{C}_{6} \mathrm{H}_{3}\left(\mathrm{C}_{6} \mathrm{H}_{4} \mathrm{SiPh}_{2} \mathrm{O}\right)_{3}\right] \mathrm{Mo}\left(\mathrm{C}_{3} \mathrm{Bu}_{3}\right)\left(\mathrm{MT}_{\mathrm{d}} 2\right)$

Cat7 $(78 \mathrm{mg} / 0.073 \mathrm{mmol})$ was dissolved into $1.2 \mathrm{ml}$ of $\mathrm{CH}_{2} \mathrm{Cl}_{2}$ and 5decyne $(101 \mathrm{mg} / 0.733 \mathrm{mmol})$ leading to a dark brown solution. The solution was stored at $-37^{\circ} \mathrm{C}$ for 6 months to give crystalline material of sufficient quality to acquire X-ray diffraction data. Not all shifts of $\mathbf{M T}_{\mathbf{d}} \mathbf{2}$ in the ${ }^{1} \mathrm{H}$ and ${ }^{13} \mathrm{C}$ NMR couple be assigned due to signal broadening and overlaps with 5-decyne and Cat7. ${ }^{1} \mathrm{H}$ NMR (500 $\left.\mathrm{MHz}, \quad \mathrm{C}_{6} \mathrm{D}_{6}, \quad 25 \quad{ }^{\circ} \mathrm{C}\right): \quad 0.62 \quad\left(9 \mathrm{H}, \quad \mathrm{t}, \quad{ }^{3} \mathrm{~J}_{\mathrm{HH}}=7.5 \quad \mathrm{~Hz}\right.$, $\mathrm{Mo}\left(\mathrm{CCH}_{2} \mathrm{CH}_{2} \mathrm{CH}_{2} \mathrm{CH}_{3}\right)_{3}, \quad 0.99 \quad\left(6 \mathrm{H}, \quad \mathrm{t}, \quad{ }^{3} \mathrm{~J}_{\mathrm{HH}}=7.5 \mathrm{~Hz}\right.$, $\mathrm{Mo}\left(\mathrm{CCH}_{2} \mathrm{CH}_{2} \mathrm{CH}_{2} \mathrm{CH}_{3}\right)_{3}, \quad 1.18-1.29\left(12 \mathrm{H}, \quad \mathrm{m},{ }^{3} \mathrm{~J}_{\mathrm{HH}}=7.5 \mathrm{~Hz}\right.$, $\mathrm{Mo}\left(\mathrm{CCH}_{2} \mathrm{CH}_{2} \mathrm{CH}_{2} \mathrm{CH}_{3}\right)_{3} .{ }^{13} \mathrm{C}\left\{{ }^{1} \mathrm{H}\right\}$ NMR $\left(125.77 \mathrm{MHz}, \mathrm{C}_{6} \mathrm{D}_{6}, 25{ }^{\circ} \mathrm{C}\right)$ : 150.6 (Ar), 144.5 (Ar), 137.9 (Ar), $137.7(A r), 137.3(A r), 135.6(A r), 129.6(A r), 129.3(A r), 128.8$ (Ar), $128.6(A r), 125.7 \quad(A r), 125.7 \quad(A r), 83.4 \quad\left(C_{3} \mathrm{Bu}_{3}\right), 33.4 \quad\left(\mathrm{CH}_{2} \mathrm{CH}_{2} \mathrm{CH}_{2} \mathrm{CH}_{3}\right), 26.1$ $\left(\mathrm{CH}_{2} \mathrm{CH}_{2} \mathrm{CH}_{2} \mathrm{CH}_{3}\right), 22.8\left(\mathrm{CH}_{2} \mathrm{CH}_{2} \mathrm{CH}_{2} \mathrm{CH}_{3}\right), 14.2\left(\mathrm{CCH}_{2} \mathrm{CH}_{2} \mathrm{CH}_{2} \mathrm{CH}_{3}\right)$.

\section{$\left[\mathrm{C}_{6} \mathrm{H}_{3}\left(\mathrm{C}_{6} \mathrm{H}_{4} \mathrm{SiPh}_{2} \mathrm{O}\right)_{3}\right] \mathrm{W}\left(\mathrm{C}_{3} \mathrm{MesEt}_{2}\right)(\mathrm{MCBD} 2)$}

Cat3 $(73 \mathrm{mg} / 0.06 \mathrm{mmol})$ was suspended into $5 \mathrm{ml}$ of pentane and to this was added 3-hexyne $(10 \mathrm{mg} / 0.120 \mathrm{mmol})$, without stirring, resulting in a purple solution. Storage of this still solution at room temperature for $2 \mathrm{hr}$ led to the deposition of crystalline material. Storage at $-37{ }^{\circ} \mathrm{C}$ led to additional crystalline material. Yield $=32 \mathrm{mg}$ (41\%). ${ }^{1} \mathrm{H}$ NMR (400 MHz, $\left.\mathrm{C}_{6} \mathrm{D}_{6}, 25^{\circ} \mathrm{C}\right): 0.33$ (3H, br s, $\mathrm{CH}_{2} \mathrm{CH}_{3}$ ), $0.70\left(3 \mathrm{H}, \mathrm{br} \mathrm{s}, \mathrm{CH}_{2} \mathrm{CH}_{3}\right), 1.52\left(6 \mathrm{H}, \mathrm{br} \mathrm{s}, o-\mathrm{CH}_{3}\right), 2.07\left(3 \mathrm{H}, \mathrm{s}, p-\mathrm{CH}_{3}\right)$, $2.63\left(2 \mathrm{H}\right.$, br s, $\left.\mathrm{CH}_{2} \mathrm{CH}_{3}\right), 3.09\left(2 \mathrm{H}\right.$, br s, $\left.\mathrm{CH}_{2} \mathrm{CH}_{3}\right), 6.60(2 \mathrm{H}, \mathrm{s}$, $\left.\mathrm{C}_{6} \mathrm{H}_{2} \mathrm{Me}_{3}\right), 6.93-7.12(22 \mathrm{H}, \mathrm{m}, \mathrm{Ar}-\mathrm{H}), 7.30-7.90(20 \mathrm{H}, \mathrm{m}, \mathrm{Ar}-\mathrm{H}), 8.01$ $(2 \mathrm{H}$, br s, Ar- $H), 8.39(1 \mathrm{H}$, br s, Ar- $H)$.

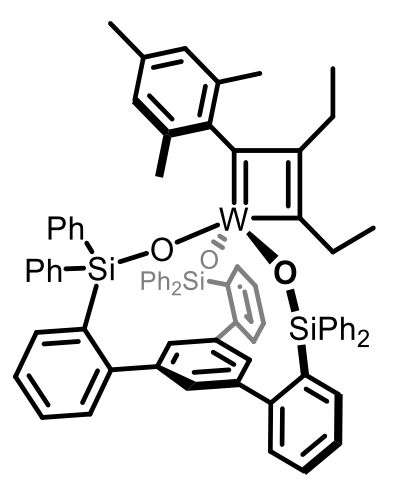




\section{$\left.\left[\mathrm{C}_{6} \mathrm{H}_{3}\left(\mathrm{C}_{6} \mathrm{H}_{4} \mathrm{SiEt}_{2} \mathrm{O}\right)_{3}\right] \mathrm{W}\left(\mathrm{C}_{3} \mathrm{MesEt}\right)_{2}\right)(\mathrm{MCBD3})$}

Cat5 (160 mg/0.151 mmol) was dissolved into $3 \mathrm{ml}$ of pentane and to this solution was added 3-hexyne $(13 \mathrm{mg} / 0.157 \mathrm{mmol})$ at room temperature, leading to a purple solution. was allowed to stir for $1.5 \mathrm{~h}$ while warming to room-temperature. Storage of the solution overnight at $-37{ }^{\circ} \mathrm{C}$ lead to the deposition of purple crystalline material. Yield $=$ $131 \mathrm{mg}(86.1 \%) .{ }^{1} \mathrm{H}$ NMR (400 MHz, $\left.\mathrm{C}_{6} \mathrm{D}_{6}, 25{ }^{\circ} \mathrm{C}\right): 0.70(15 \mathrm{H}$, br s, $\left.\mathrm{SiCH}_{2} \mathrm{CH}_{3} \& \mathrm{CH}_{2} \mathrm{CH}_{3}\right), 0.85-1.05\left(21 \mathrm{H}\right.$, br s, $\left.\mathrm{SiCH}_{2} \mathrm{CH}_{3} \& \mathrm{CH}_{2} \mathrm{CH}_{3}\right)$, $2.18\left(3 \mathrm{H}, \mathrm{s}, p-\mathrm{CH}_{3}\right), 2.29\left(6 \mathrm{H}\right.$, br s, $\left.o-\mathrm{CH}_{3}\right), 2.93\left(2 \mathrm{H}\right.$, br s, $\left.\mathrm{CH}_{2} \mathrm{CH}_{3}\right)$, $3.27\left(2 \mathrm{H}\right.$, br s, $\left.\mathrm{CH}_{2} \mathrm{CH}_{3}\right), 6.91\left(2 \mathrm{H}, \mathrm{s}, \mathrm{C}_{6} \mathrm{H}_{2} \mathrm{Me}_{3}\right), 7.21(3 \mathrm{H}$, br s, Ar$H), 7.32(3 \mathrm{H}$, br s, Ar-H), 7.43 (3H, br s, Ar-H), 7.64 (3H, br s, Ar- $H)$.

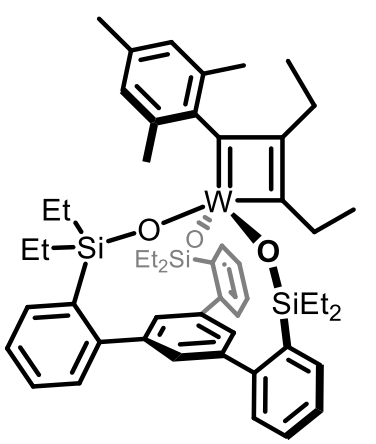

Decompositon of MCBD3 to MCBD5 and 1-mesityl-1-butyne frustrated our attempts at ${ }^{13} \mathrm{C}$ collection.

\section{$\left[\mathrm{C}_{6} \mathrm{H}_{3}\left(\mathrm{C}_{6} \mathrm{H}_{4} \mathrm{SiPh}_{2} \mathrm{O}\right)_{3}\right] \mathrm{W}\left(\mathrm{C}_{3} \mathrm{Et}_{3}\right)$ (MCBD4)}

Cat3 $(70 \mathrm{mg} / 0.058 \mathrm{mmol})$ was dissolved into $0.6 \mathrm{ml}$ of $\mathrm{C}_{6} \mathrm{D}_{6}$ and 3hexyne $(12 \mathrm{mg} / 0.148 \mathrm{mmol})$ was added leading to a purple solution. The reaction was heated to $60{ }^{\circ} \mathrm{C}$ for 15 minutes leading to a reddish-maroon solution. All volatiles were removed under reduced pressure and the resulting oily-solid was dissolved into dichloromethane. Crystalline material was produced via slow-evaporation at $-37^{\circ} \mathrm{C}$ overnight. Yield $=57.5 \mathrm{mg}(82.3 \%) .{ }^{1} \mathrm{H} \mathrm{NMR}\left(500 \mathrm{MHz}, \mathrm{CD}_{2} \mathrm{Cl}_{2},-70{ }^{\circ} \mathrm{C}\right):-0.28(3 \mathrm{H}, \mathrm{t}$, $\left.{ }^{3} \mathrm{~J}_{\mathrm{HH}}=6.0 \mathrm{~Hz}, \alpha^{\prime}-\mathrm{CCH}_{2} \mathrm{CH}_{3}\right), 0.96\left(3 \mathrm{H}, \mathrm{t},{ }^{3} \mathrm{~J}_{\mathrm{HH}}=6.0 \mathrm{~Hz}, \alpha-\mathrm{CCH}_{2} \mathrm{CH}_{3}\right)$, $1.22\left(3 \mathrm{H}, \mathrm{t},{ }^{3} \mathrm{~J}_{\mathrm{HH}}=6.0 \mathrm{~Hz}, \beta-\mathrm{CCH}_{2} \mathrm{CH}_{3}\right), 1.82\left(2 \mathrm{H}, \mathrm{q},{ }^{3} \mathrm{~J}_{\mathrm{HH}}=6.0 \mathrm{~Hz}, \alpha{ }^{\prime}-\right.$

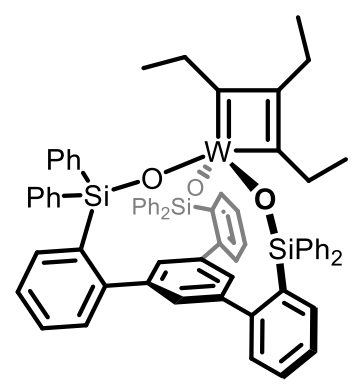

$\left.\mathrm{CCH}_{2} \mathrm{CH}_{3}\right), 3.29\left(2 \mathrm{H}, \mathrm{q},{ }^{3} \mathrm{~J}_{\mathrm{HH}}=6.0 \mathrm{~Hz}, \beta-\mathrm{CCH}_{2} \mathrm{CH}_{3}\right), 4.16\left(2 \mathrm{H}, \mathrm{q},{ }^{3} \mathrm{~J}_{\mathrm{HH}}=6.0 \mathrm{~Hz}, \alpha-\mathrm{CCH}_{2} \mathrm{CH}_{3}\right)$, 6.82-7.86 (45H, m, Ar $).{ }^{13} \mathrm{C}\left\{{ }^{1} \mathrm{H}\right\}$ NMR $\left(125 \mathrm{MHz}, \mathrm{CD}_{2} \mathrm{Cl}_{2},-70{ }^{\circ} \mathrm{C}\right): 234.78\left(\alpha^{\prime}-\mathrm{CCH}_{2} \mathrm{CH}_{3}\right), 229.89$ $\left(\alpha-\mathrm{CCH}_{2} \mathrm{CH}_{3}\right), 148.72(\mathrm{Ar}), 148.28(\mathrm{Ar}), 147.65(\mathrm{Ar}), 145.96(\mathrm{Ar}), 143.23(\mathrm{Ar}), 142.82(\mathrm{Ar})$, 140.33 (Ar), $139.69(A r), 139.40(A r), 139.23(A r), 138.93(A r), 138.79\left(\beta-C_{C H} \mathrm{CH}_{3}\right), 138.00$ (Ar), 137.25 (Ar), $136.94(A r), 136.35(A r), 135.37$ (Ar), $135.20(A r), 134.51(A r), 134.31(A r)$, 134.12 (Ar), 133.64 (Ar), 130.73 (Ar), 130.31 (Ar), 129.84 (Ar), 129.57 (Ar), 129.22 (Ar), 128.96 (Ar), $128.84(A r), 128.40(A r), 127.73(A r), 127.73$ (Ar), 127.49 (Ar), 127.27 (Ar), $127.21(A r)$, $127.05(A r), 126.19(A r), 125.91(A r), 125.22(A r), 125.13(A r), 1215.57(A r), 30.07(\alpha-$ $\left.\mathrm{CCH}_{2} \mathrm{CH}_{3}\right), 28.17\left(\alpha^{\prime}-\mathrm{CCH}_{2} \mathrm{CH}_{3}\right), 26.03\left(\beta-\mathrm{CCH}_{2} \mathrm{CH}_{3}\right), \quad 16.07\left(\alpha-\mathrm{CCH}_{2} \mathrm{CH}_{3}\right), 14.20\left(\alpha^{\prime}-\right.$ $\left.\mathrm{CCH}_{2} \mathrm{CH}_{3}\right), 13.89\left(\beta-\mathrm{CCH}_{2} \mathrm{CH}_{3}\right)$.

\section{$\left[\mathrm{C}_{6} \mathrm{H}_{3}\left(\mathrm{C}_{6} \mathrm{H}_{4} \mathrm{SiEt}_{2} \mathrm{O}\right)_{3}\right] \mathrm{W}\left(\mathrm{C}_{3} \mathrm{Et}_{3}\right)$ (MCBD5)}

Cat5 $(44 \mathrm{mg} / 0.036 \mathrm{mmol})$ was dissolved into $0.6 \mathrm{ml}$ of $\mathrm{C}_{6} \mathrm{D}_{6}$ and 3hexyne $(15 \mathrm{mg} / 0.181 \mathrm{mmol})$ was added leading to a purple solution. The reaction proceeded overnight at which point all volatiles were removed under reduced pressure. The resulting purple solid was dissolved into minimal pentane and stored at $-37^{\circ} \mathrm{C}$ overnight. Solvent was removed and the purple crystalline material was dried under reduced pressure. Yield $=18 \mathrm{mg}(53 \%)$. ${ }^{1} \mathrm{H}$ NMR $\left(500 \mathrm{MHz}, \mathrm{C}_{6} \mathrm{D}_{6}, 25^{\circ} \mathrm{C}\right): 0.12(2 \mathrm{H}, \mathrm{q}$, $\left.{ }^{3} \mathrm{~J}_{\mathrm{HH}}=10.0 \mathrm{~Hz}, \mathrm{SiCH}_{2} \mathrm{CH}_{3}\right), 0.40\left(3 \mathrm{H}, \mathrm{t},{ }^{3} \mathrm{~J}_{\mathrm{HH}}=7.6 \mathrm{~Hz}, \alpha{ }^{\prime}-\mathrm{CCH}_{2} \mathrm{CH}_{3}\right)$,

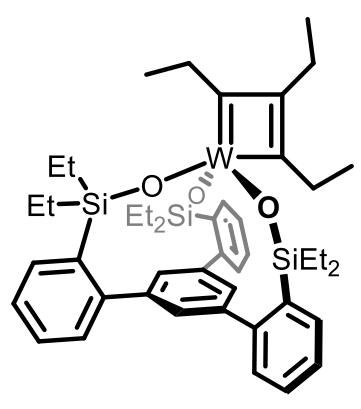


$0.60\left(6 \mathrm{H}, \mathrm{t},{ }^{3} \mathrm{~J}_{\mathrm{HH}}=4.4 \mathrm{~Hz}, \mathrm{SiCH}_{2} \mathrm{CH}_{3}\right), 0.74\left(3 \mathrm{H}, \mathrm{s},{ }^{3} \mathrm{~J}_{\mathrm{HH}}=7.6 \mathrm{~Hz}, \beta-\mathrm{CCH}_{2} \mathrm{CH}_{3}\right), 0.86-0.91(10 \mathrm{H}$, $\mathrm{m}, \mathrm{SiCH}_{2} \mathrm{CH}_{3}$ and $\left.\mathrm{SiCH}_{2} \mathrm{CH}_{3}\right), 1.15\left(2 \mathrm{H}, \mathrm{m},{ }^{3} \mathrm{~J}_{\mathrm{HH}}=7.5 \mathrm{~Hz}, \mathrm{SiCH}_{2} \mathrm{CH}_{3}\right), 1.25\left(4 \mathrm{H}, \mathrm{m},{ }^{3} \mathrm{~J}_{\mathrm{HH}}=10\right.$ $\left.\mathrm{Hz}, \mathrm{SiCH}_{2} \mathrm{CH}_{3}\right), 1.39\left(6 \mathrm{H}, \mathrm{t},{ }^{3} \mathrm{~J}_{\mathrm{HH}}=7.5 \mathrm{~Hz}, \mathrm{SiCH}_{2} \mathrm{CH}_{3}\right), 1.44\left(3 \mathrm{H}, \mathrm{t},{ }^{3} \mathrm{~J}_{\mathrm{HH}}=7.5 \mathrm{~Hz}, \beta-\mathrm{CCH}_{2} \mathrm{CH}_{3}\right)$, $2.96\left(2 \mathrm{H}, \mathrm{q},{ }^{3} \mathrm{~J}_{\mathrm{HH}}=7.5 \mathrm{~Hz}, \beta-\mathrm{CCH}_{2} \mathrm{CH}_{3}\right), 3.07\left(2 \mathrm{H}, \mathrm{q},{ }^{3} \mathrm{~J}_{\mathrm{HH}}=7.5 \mathrm{~Hz}, \alpha-\mathrm{CCH}_{2} \mathrm{CH}_{3}\right), 3.89(2 \mathrm{H}, \mathrm{q}$, $\left.{ }^{3} \mathrm{~J}_{\mathrm{HH}}=7.5 \mathrm{~Hz}, \alpha-\mathrm{CCH}_{2} \mathrm{CH}_{3}\right), 7.17-7.23(4 \mathrm{H}, \mathrm{m}, \mathrm{Ar}-H), 7.29-7.33(3 \mathrm{H}, \mathrm{m}, \mathrm{Ar}-H), 7.37\left(2 \mathrm{H}, \mathrm{d},{ }^{3} \mathrm{~J}_{\mathrm{HH}}\right.$ $=5.0 \mathrm{~Hz}, \operatorname{Ar}-H), 7.53\left(1 \mathrm{H}, \mathrm{d},{ }^{3} \mathrm{~J}_{\mathrm{HH}}=5.0 \mathrm{~Hz}, \mathrm{Ar}-H\right), 7.61\left(2 \mathrm{H}, \mathrm{d},{ }^{3} \mathrm{~J}_{\mathrm{HH}}=10 \mathrm{~Hz}, \mathrm{Ar}-H\right), 7.64(2 \mathrm{H}$, $\left.\mathrm{d},{ }^{4} \mathrm{~J}_{\mathrm{HH}}=5.0 \mathrm{~Hz}, \mathrm{C}_{6} \mathrm{H}_{3}\right), 7.96\left(1 \mathrm{H}, \mathrm{t},{ }^{4} \mathrm{~J}_{\mathrm{HH}}=5.0 \mathrm{~Hz}, \mathrm{C}_{6} \mathrm{H}_{3}\right) .{ }^{13} \mathrm{C}\left\{{ }^{1} \mathrm{H}\right\} \mathrm{NMR}\left(125.77 \mathrm{MHz}, \mathrm{C}_{6} \mathrm{D}_{6}, 25\right.$ $\left.{ }^{\circ} \mathrm{C}\right): 229.38\left(\alpha \cdot-\mathrm{CCH}_{2} \mathrm{CH}_{3}\right), 221.95\left(\alpha-\mathrm{CCH}_{2} \mathrm{CH}_{3}\right), 149.43(\mathrm{Ar}), 149.23(\mathrm{Ar}), 146.00(\mathrm{Ar}), 143.23$ (Ar), $140.11(A r), 138.21(A r), 135.15(A r), 133.91(A r), 131.73\left(\beta-C_{C H} \mathrm{CH}_{3}\right), 131.02,129.33$ (Ar), $128.99(A r), 126.61(A r), 126.28(A r), 30.17\left(\alpha-\mathrm{CCH}_{2} \mathrm{CH}_{3}\right), 29.92\left(\alpha-\mathrm{CCH}_{2} \mathrm{CH}_{3}\right), 24.81(\beta-$ $\left.\left.\mathrm{CCH}_{2} \mathrm{CH}_{3}\right), 17.23\left(\alpha-\mathrm{CCH}_{2} \mathrm{CH}_{3}\right), 15.09(\alpha)-\mathrm{CCH}_{2} \mathrm{CH}_{3}\right), 14.23\left(\beta-\mathrm{CCH}_{2} C_{3}\right), 13.37\left(\mathrm{SiCH}_{2} \mathrm{CH}_{3}\right)$, 10.39 $\left(\mathrm{SiCH}_{2} \mathrm{CH}_{3}\right), 10.25\left(\mathrm{SiCH}_{2} \mathrm{CH}_{3}\right), 8.55\left(\mathrm{SiCH}_{2} \mathrm{CH}_{3}\right), 7.98\left(\mathrm{SiCH}_{2} \mathrm{CH}_{3}\right), 7.70\left(\mathrm{SiCH}_{2} \mathrm{CH}_{3}\right)$, $7.07\left(\mathrm{SiCH}_{2} \mathrm{CH}_{3}\right)$.

\section{[ $\left.\mathrm{C}_{6} \mathrm{H}_{3}\left(\mathrm{C}_{6} \mathrm{H}_{4} \mathrm{SiPh}_{2} \mathrm{O}\right)_{3}\right] \mathrm{W}\left(\mathrm{C}_{3} \mathrm{MesTolCH}\right)$ (MCBD6)}

Cat3 $(45 \mathrm{mg} / 0.037 \mathrm{mmol})$ was added to $10 \mathrm{ml}$ of pentane to give a yellow suspension. $p$-tolylpropyne $(24 \mathrm{mg} / 0.185 \mathrm{mmol})$ was added at room temperature resulting in the solution darkening to purplebrown. Dark purple, square crystals began forming after 5 minutes and continued to form for $2 \mathrm{hr}$ at which point the solvent was decanted away and the solids dried under reduced pressure. Yield $=24.2 \mathrm{mg}(48.6 \%) .{ }^{1} \mathrm{H}$ NMR $\left(500 \mathrm{MHz}, \mathrm{C}_{6} \mathrm{D}_{6}, 25^{\circ} \mathrm{C}\right): 1.46(3 \mathrm{H}$, br s, $\left.\mathrm{CH}_{3}\right), 1.87\left(3 \mathrm{H}\right.$, br s, $\left.\mathrm{CH}_{3}\right), 2.12\left(6 \mathrm{H}\right.$, br s, $\left.o-\mathrm{CH}_{3}\right), 2.23(3 \mathrm{H}$, s, $\left.p-\mathrm{CH}_{3}\right), 6.43(1 \mathrm{H}$, br s, Ar- $H), 6.68(2 \mathrm{H}$, br s, Ar- $H), 6.92-7.09$ $(24 \mathrm{H}, \mathrm{m}, \mathrm{Ar}-H), 7.31$ (4 H, s, Ar-H), 7.65 (4 H, m, Ar- $H), 7.98$ (1

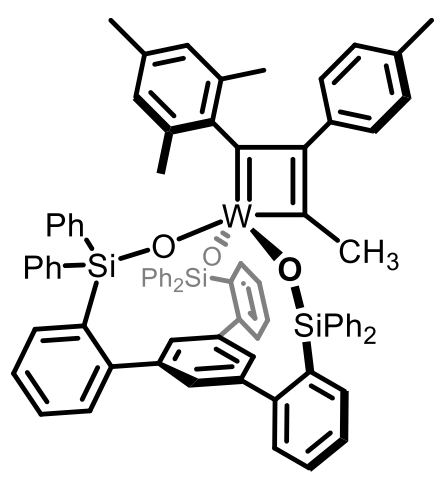
$\mathrm{H}, \mathrm{d}, \mathrm{Ar}-H), 8.08(2 \mathrm{H}, \mathrm{s}, \mathrm{Ar}-H) .{ }^{13} \mathrm{C}\left\{{ }^{1} \mathrm{H}\right\} \mathrm{NMR}\left(125.77 \mathrm{MHz}, \mathrm{C}_{6} \mathrm{D}_{6}, 25{ }^{\circ} \mathrm{C}\right): 231.50\left(\alpha-C_{3}\right), 228$. $02\left(\alpha-C_{3}\right) 150.07(A r), 144.08\left(\beta-C_{3}\right), 141.87(A r), 139.79(A r), 139.58(A r), 139.31(A r), 137.89$ (Ar), $137.78(A r), 137.68(A r), 137.56(A r), 137.49$ (Ar), 137.01 (Ar), $136.68(A r), 136.40(A r)$, 136.07 (Ar), 135.98 (Ar), 135.63 (Ar), 135.02 (Ar), 131.86 (Ar), 131.43 (Ar), 126.46 (Ar), 125.71 (Ar), $122.00(A r), 21.45\left(p-C \mathrm{H}_{3}\right), 20.96\left(o-C \mathrm{H}_{3}\right), 20.81\left(p-\mathrm{CH}_{3}\right), 20.27\left(\mathrm{C}_{2} C_{3}\right)$.

\section{$\left[\mathrm{C}_{6} \mathrm{H}_{3}\left(\mathrm{C}_{6} \mathrm{H}_{4} \mathrm{SiEt}_{2} \mathrm{O}\right)_{3}\right] \mathrm{W} \equiv \mathrm{CMes}(\mathrm{PhCN})(\mathrm{Cat5} \bullet \mathrm{PhCN})$}

Cat5 $(60 \mathrm{mg} / 0.065 \mathrm{mmol})$ was dissolved into $5 \mathrm{ml}$ of pentane to give a yellow solution. Benzonitrile $(7.4 \mu \mathrm{l} / 0.071 \mathrm{mmol})$ was added at room temperature without stirring. The resulting purplered solution was left undisturbed for $1 \mathrm{~h}$ leading to the deposition of dark purple-red, crystalline material. The solvent was decanted and the solids dried under reduced pressure. Yield $=52 \mathrm{mg}(78 \%)$. ${ }^{1} \mathrm{H}$ NMR (500 MHz, $\left.\mathrm{C}_{6} \mathrm{D}_{6}, 25^{\circ} \mathrm{C}\right): 0.99\left(12 \mathrm{H}, \mathrm{m}, \mathrm{SiCH}_{2} \mathrm{CH}_{3}\right)$, $1.08\left(18 \mathrm{H}, \mathrm{t},{ }^{3} \mathrm{~J}_{\mathrm{HH}}=7.5 \mathrm{~Hz}, \mathrm{SiCH}_{2} \mathrm{CH}_{3}\right), 2.33\left(3 \mathrm{H}, \mathrm{s}, p-\mathrm{CH}_{3}\right), 2.96$ $\left(6 \mathrm{H}, \mathrm{s}, o-\mathrm{CH}_{3}\right), 6.55\left(2 \mathrm{H}, \mathrm{d},{ }^{3} \mathrm{~J}_{\mathrm{HH}}=8.0 \mathrm{~Hz}, m-\mathrm{Ph}-H\right), 6.69(2 \mathrm{H}, \mathrm{t}$,

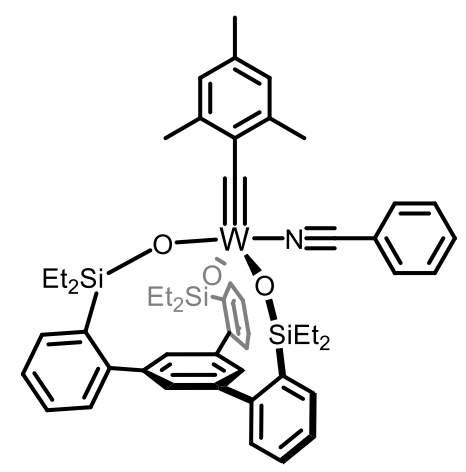
$\left.{ }^{3} \mathrm{~J}_{\mathrm{HH}}=7.5 \mathrm{~Hz}, o-\mathrm{Ph}-H\right), 6.77\left(1 \mathrm{H}, \mathrm{t},{ }^{3} \mathrm{~J}_{\mathrm{HH}}=7.5 \mathrm{~Hz}, p-\mathrm{Ph}-H\right), 6.89$

$(2 \mathrm{H}, \mathrm{s}, o-$ Mes- $H), 7.29\left(3 \mathrm{H}, \mathrm{m},{ }^{3} \mathrm{~J}_{\mathrm{HH}}=4.5 \mathrm{~Hz}, \mathrm{Ar}-H\right), 7.45\left(3 \mathrm{H}, \mathrm{m},{ }^{3} \mathrm{~J}_{\mathrm{HH}}=4.5 \mathrm{~Hz}, \mathrm{Ar}-H\right), 7.50$ $\left(3 \mathrm{H}, \mathrm{s}, \mathrm{C}_{6} \mathrm{H}_{3}\right) .{ }^{13} \mathrm{C}\left\{{ }^{1} \mathrm{H}\right\} \mathrm{NMR}\left(125.77 \mathrm{MHz}, \mathrm{C}_{6} \mathrm{D}_{6}, 25{ }^{\circ} \mathrm{C}\right): 288.40(\mathrm{~W} \equiv C), 149.63(A r), 144.23$ (Ar), $142.11(A r), 141.45$ (Ar), 137.36 (Ar), 135.72 (Ar), 134.92 (Ar), 133.47 (Ar), 132.07 (Ar), 
$130.69(A r), 128.98(A r), 128.83(A r), 128.49(A r), 128.44(A r), 126.62(A r), 126.58(A r), 110.58$ $(C \equiv \mathrm{N}), 20.95\left(p-\mathrm{CH}_{3}\right), 20.72\left(o-\mathrm{CH}_{3}\right), 9.54\left(\mathrm{SiCH}_{2} \mathrm{CH}_{3}\right), 7.57\left(\mathrm{SiCH}_{2} \mathrm{CH}_{3}\right)$.

\section{$\left\{\left[\mathrm{C}_{6} \mathrm{H}_{3}\left(\mathrm{C}_{6} \mathrm{H}_{4} \mathrm{SiEt}_{2} \mathrm{O}\right)_{3}\right] \mathbf{W} \equiv \mathbf{N}\right\}_{2}$ (Nitride1)}

Cat5 $(40 \mathrm{mg} / 0.043 \mathrm{mmol})$ was dissolved in $0.6 \mathrm{ml}$ of $\mathrm{C}_{6} \mathrm{D}_{6}$ to give a yellow suspension. Benzonitrile $(4.7 \mu \mathrm{l} / 0.046 \mathrm{mmol})$ was added at room temperature resulting in the solution darkening to purple-red. After $3 \mathrm{~h}$ at room temperature, the solution had become yellowbrown in color and the formation of mesityl-phenylacetylene could be seen forming along with the consumption of Cat5•PhCN. After $6 \mathrm{~h}$ the solution was bright yellow and all resonances associated with Cat5•PhCN had disappeared. Slow evaporation of the solvent led

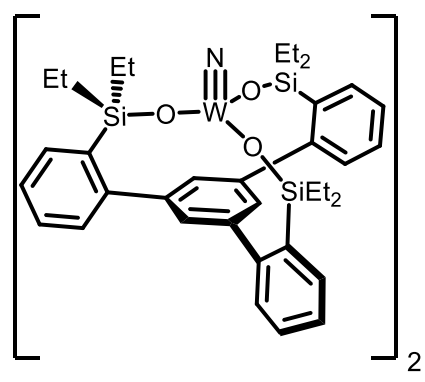
to the deposition of pale-yellow/colorless crystals of Nitride1 suitable for X-ray diffraction studies. Yield $=17 \mathrm{mg}(49 \%)$. 


\section{Supporting NMR spectra}

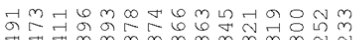

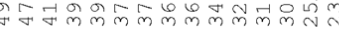

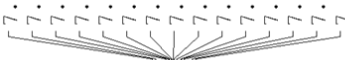

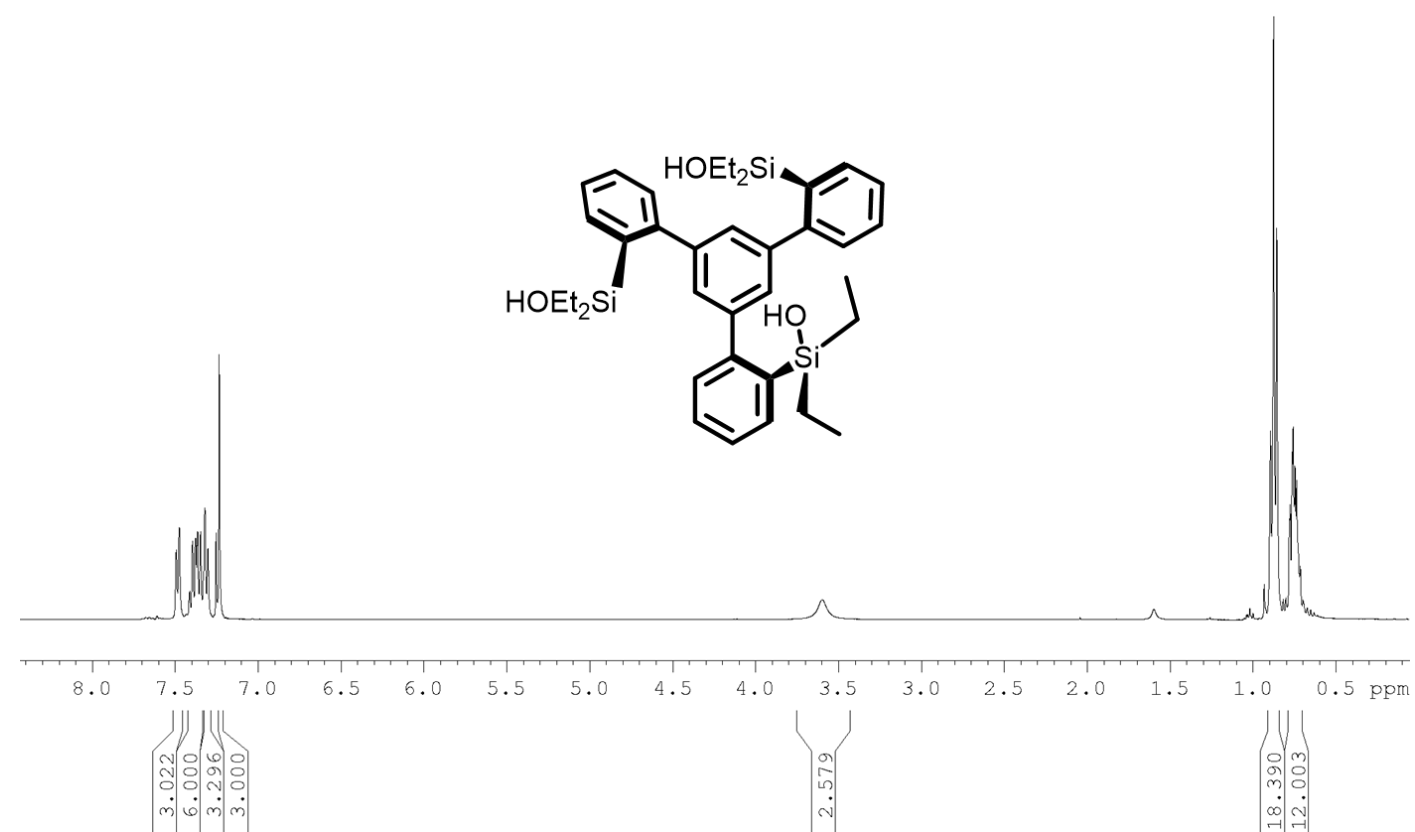

Figure S1. ${ }^{1} \mathrm{H}$ NMR $\left(400 \mathrm{MHz}, \mathrm{CDCl}_{3}, 25^{\circ} \mathrm{C}\right)$ spectrum of $\mathbf{S i P}^{\mathrm{Et}}$.
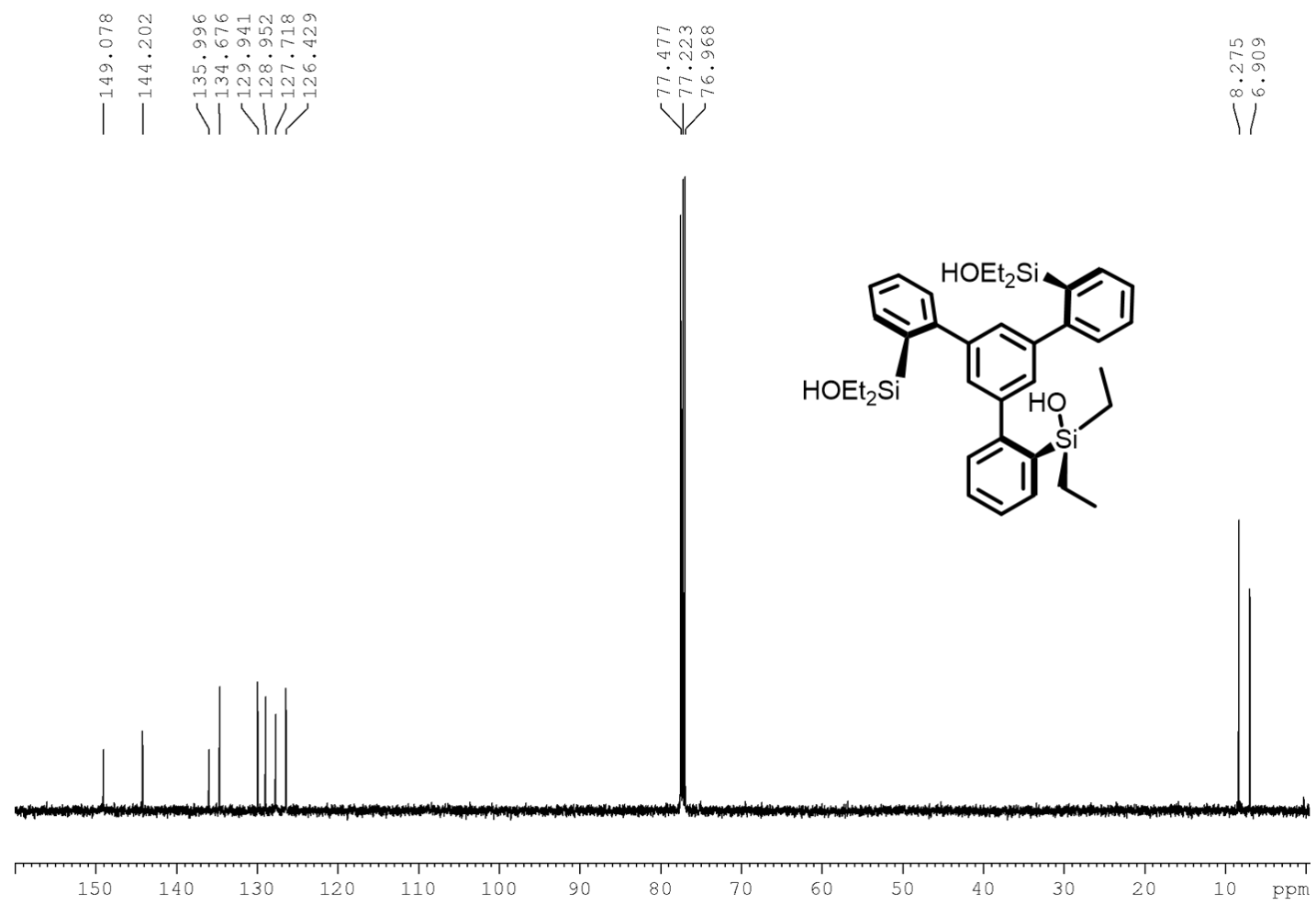

Figure S2. ${ }^{13} \mathrm{C} \mathrm{NMR}\left(125 \mathrm{MHz}, \mathrm{CDCl}_{3}, 25{ }^{\circ} \mathrm{C}\right)$ spectrum of $\mathbf{S i P}^{\mathrm{Et}}$. 


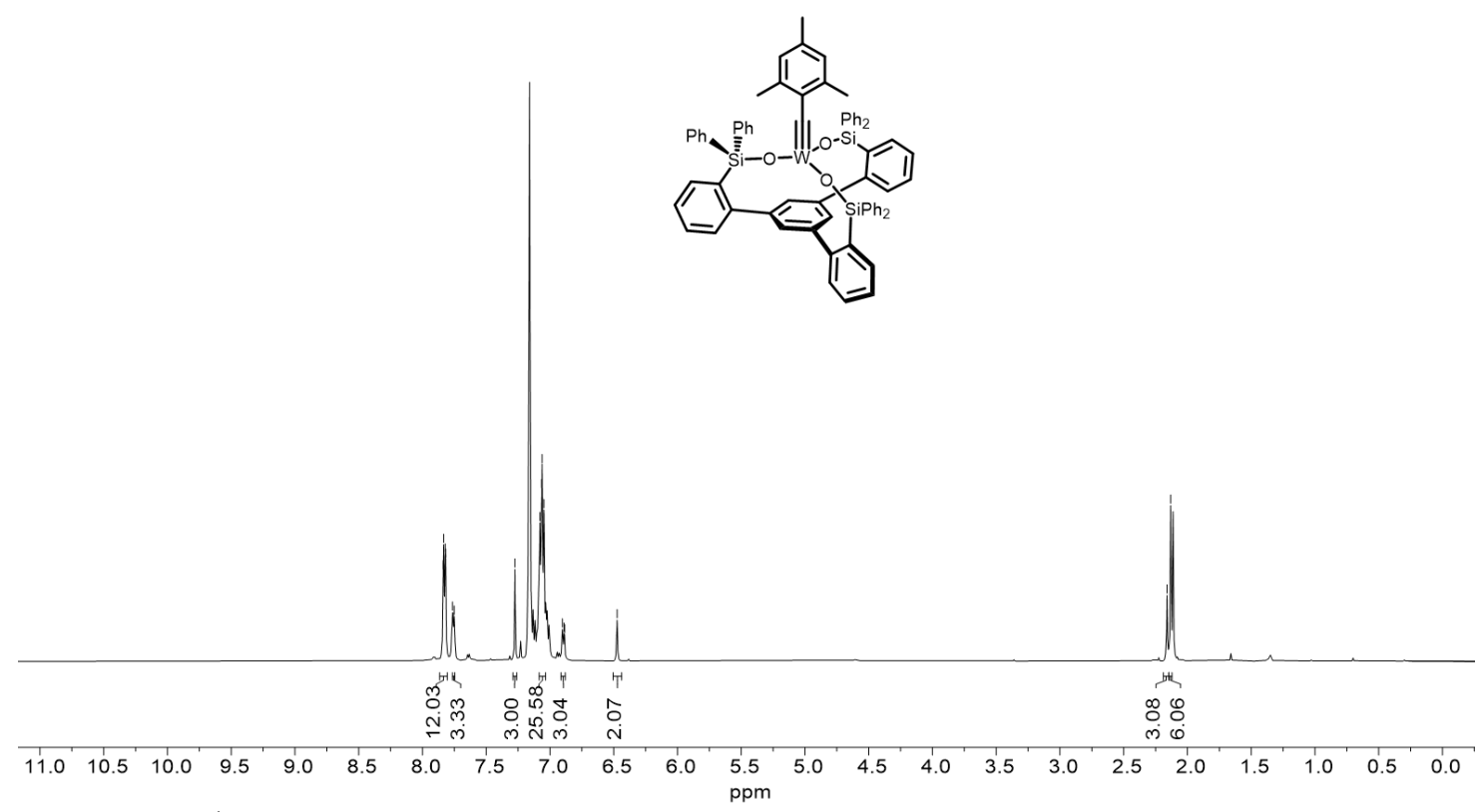

Figure S3. ${ }^{1} \mathrm{H}$ NMR $\left(500 \mathrm{MHz}, \mathrm{C}_{6} \mathrm{D}_{6}, 25{ }^{\circ} \mathrm{C}\right)$ spectrum of Cat3.
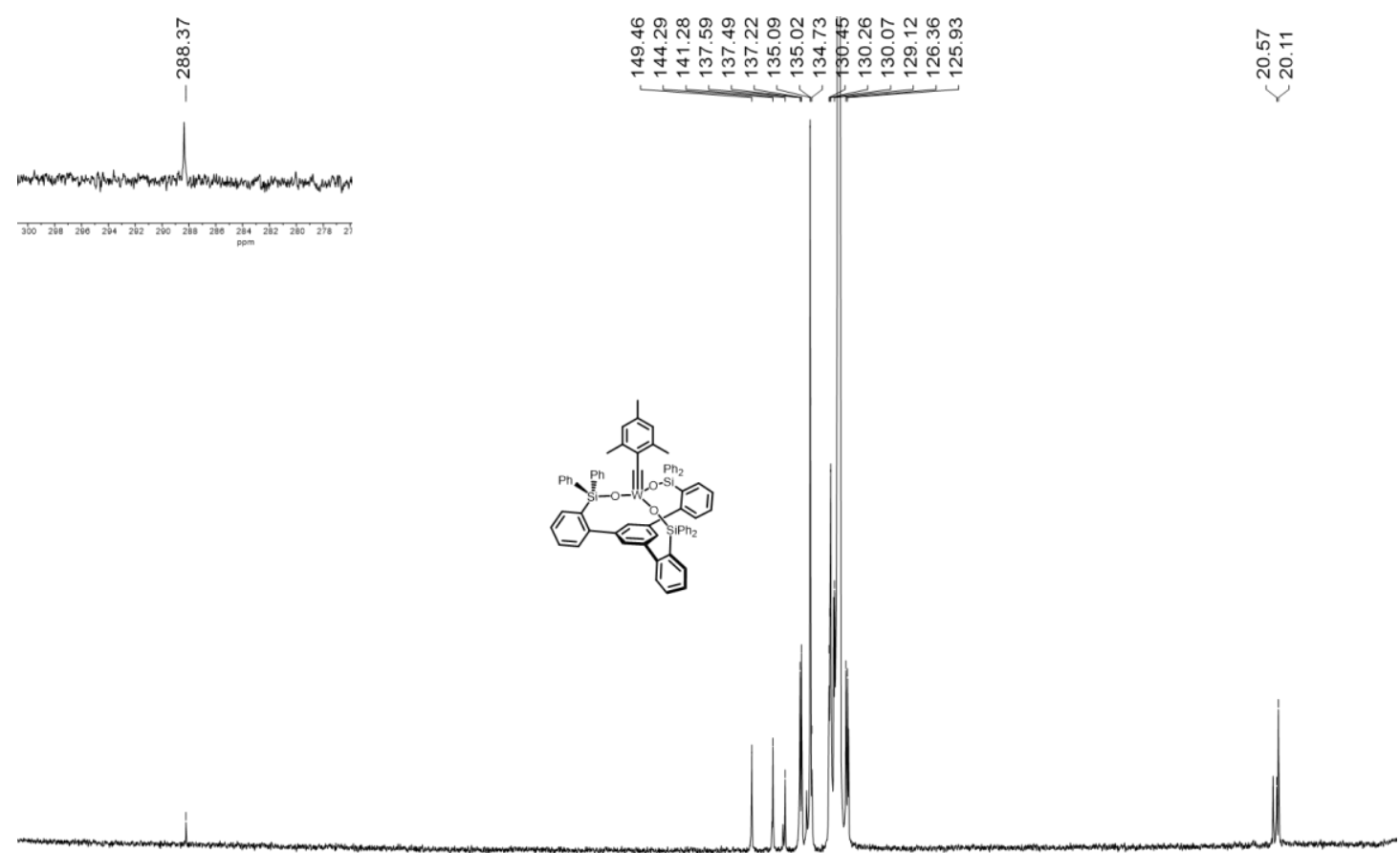

$32031030029028027026025024023022021020019018017016015014013012011010090 \quad 8070$ ppm

Figure S4. ${ }^{13} \mathrm{C}$ NMR $\left(125 \mathrm{MHz}, \mathrm{C}_{6} \mathrm{D}_{6}, 25{ }^{\circ} \mathrm{C}\right)$ spectrum of Cat3. The carbyne carbon is expanded in the inset. 


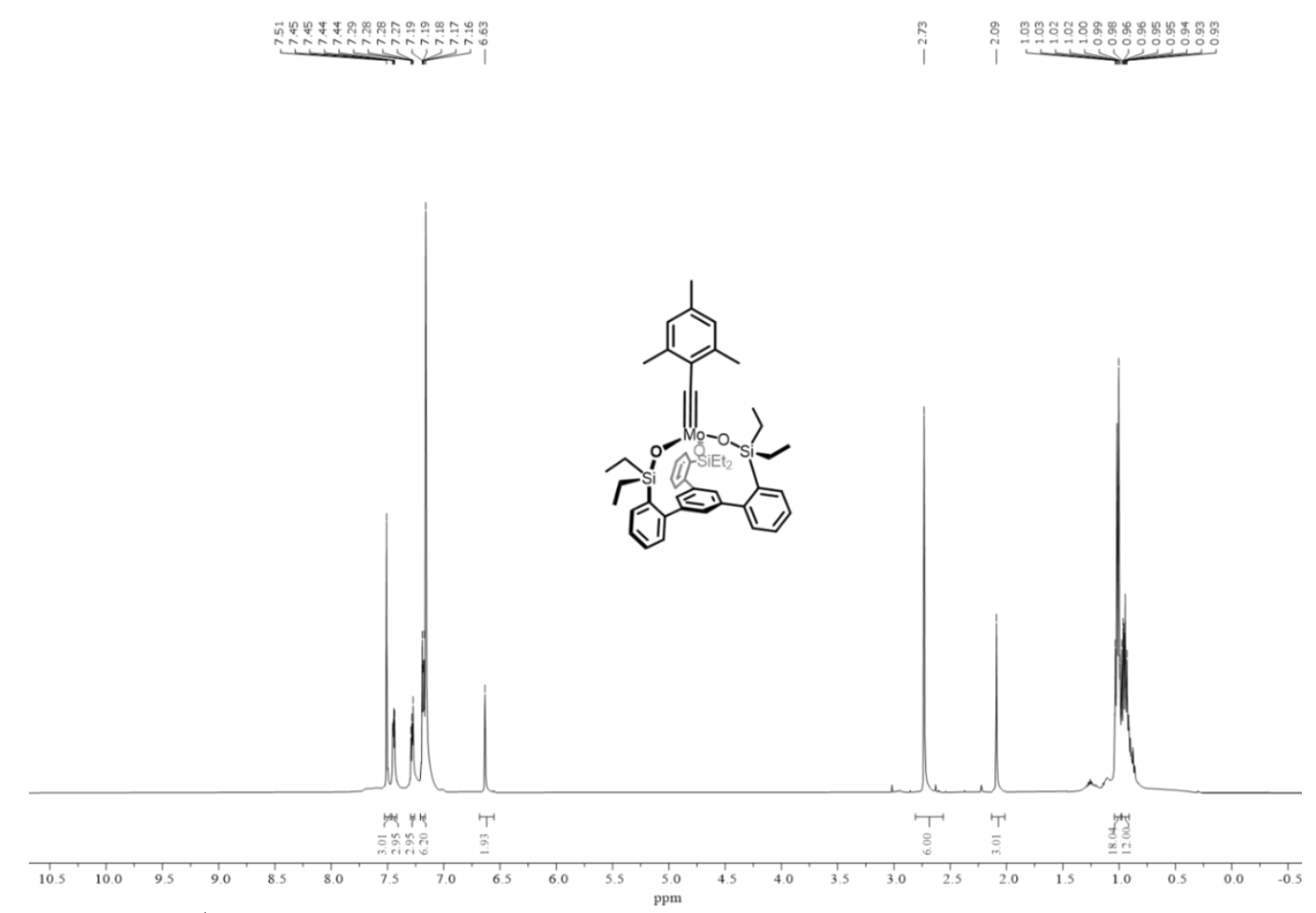

Figure S5. ${ }^{1} \mathrm{H}$ NMR $\left(400 \mathrm{MHz}, \mathrm{C}_{6} \mathrm{D}_{6}, 25^{\circ} \mathrm{C}\right)$ spectrum of Cat4.

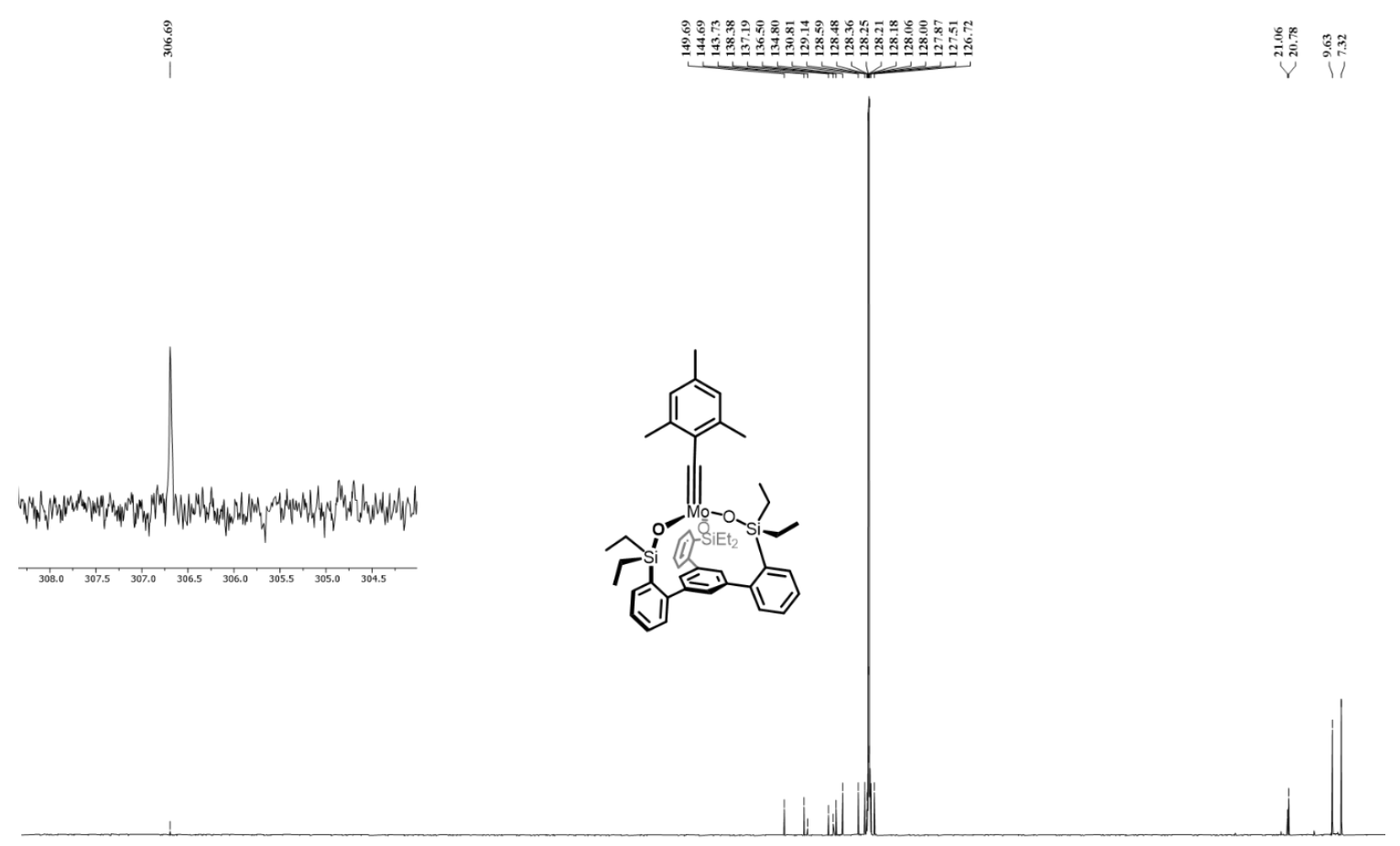

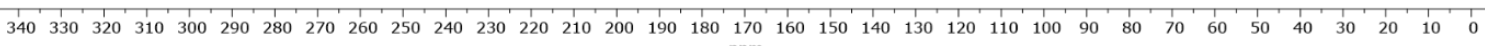

Figure S6. ${ }^{13} \mathrm{C}$ NMR $\left(125 \mathrm{MHz}, \mathrm{C}_{6} \mathrm{D}_{6}, 25^{\circ} \mathrm{C}\right)$ spectrum of Cat4. 


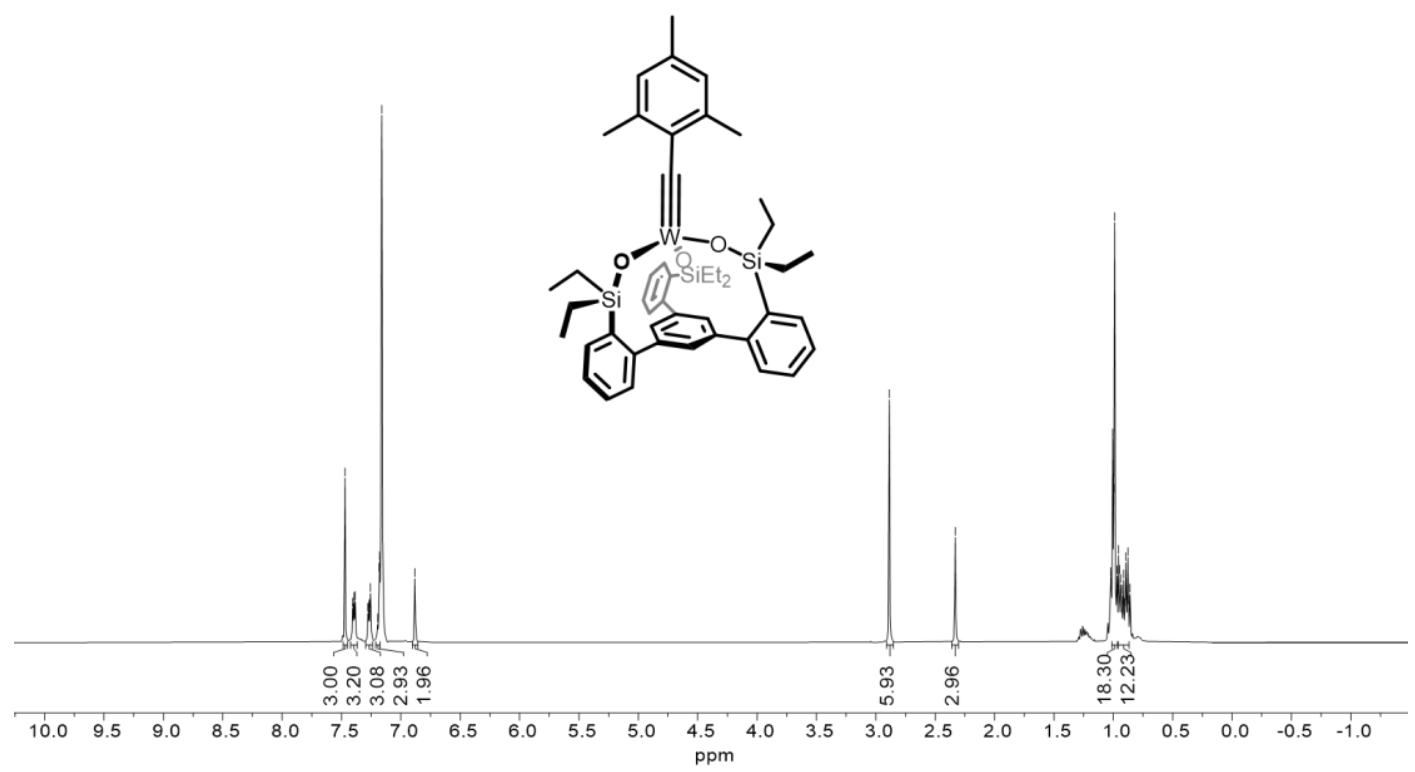

Figure S7. ${ }^{1} \mathrm{H}$ NMR $\left(500 \mathrm{MHz}, \mathrm{C}_{6} \mathrm{D}_{6}, 25^{\circ} \mathrm{C}\right)$ spectrum of Cat5.

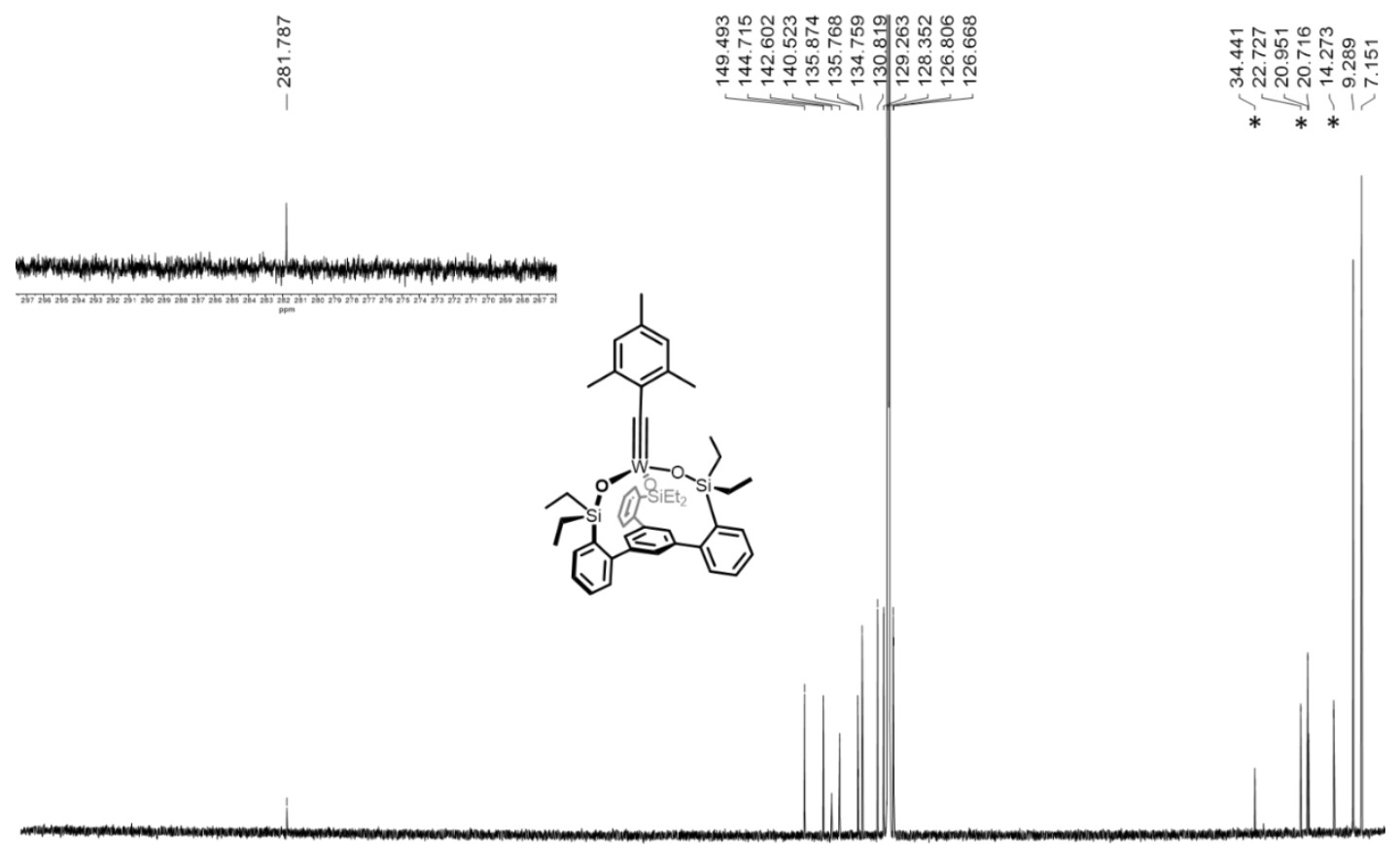

$340330320310300290280270260250240230220210200190 \begin{gathered}180 \\ \text { ppm }\end{gathered}$

Figure S8. ${ }^{13} \mathrm{C}$ NMR $\left(125 \mathrm{MHz}, \mathrm{C}_{6} \mathrm{D}_{6}, 25{ }^{\circ} \mathrm{C}\right)$ spectrum of Cat5. The carbyne carbon is expanded in the inset. Pentane impurity is denoted by $(*)$. 


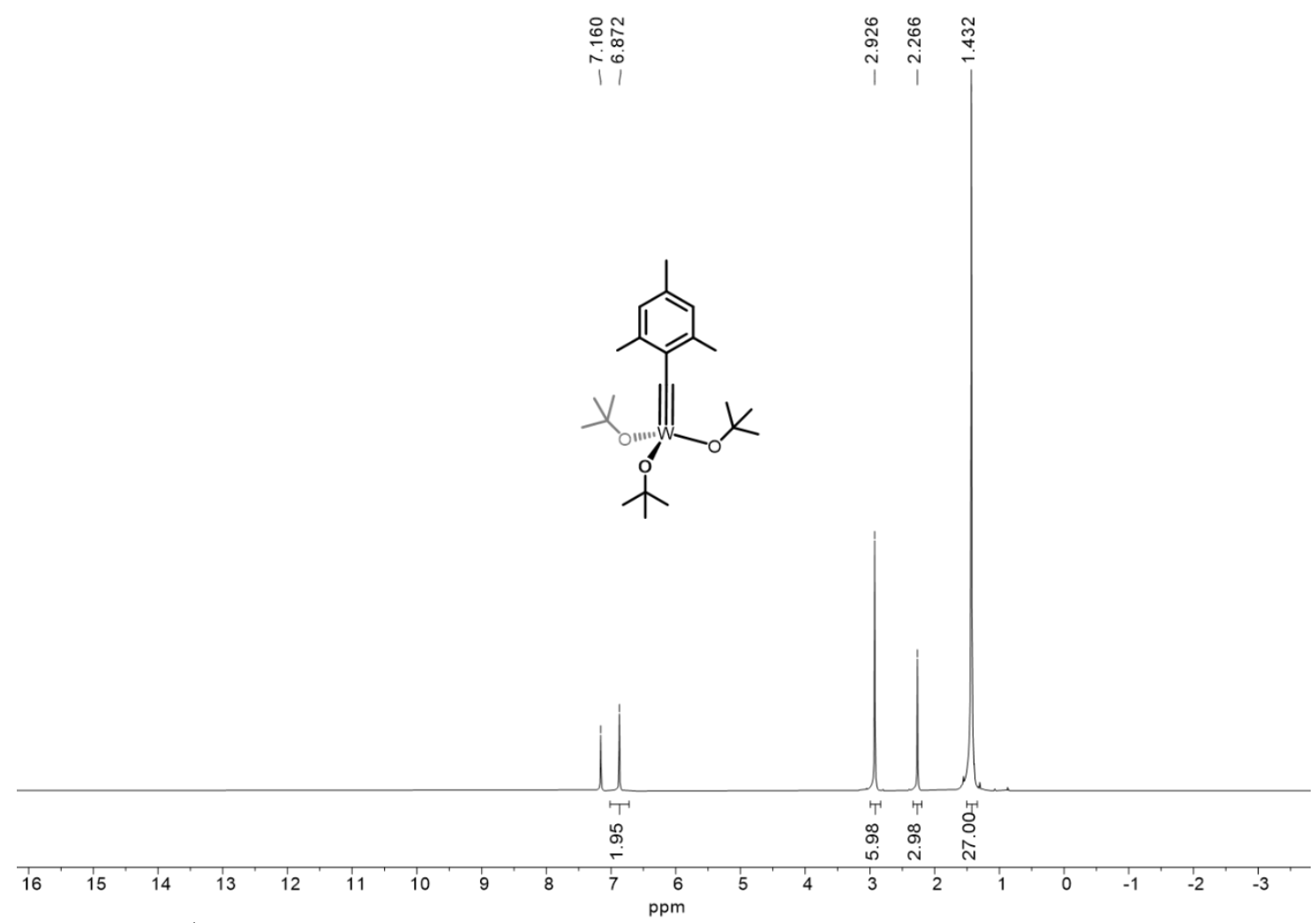

Figure S9. ${ }^{1} \mathrm{H}$ NMR $\left(500 \mathrm{MHz}, \mathrm{C}_{6} \mathrm{D}_{6}, 25^{\circ} \mathrm{C}\right)$ spectrum of Pre2.

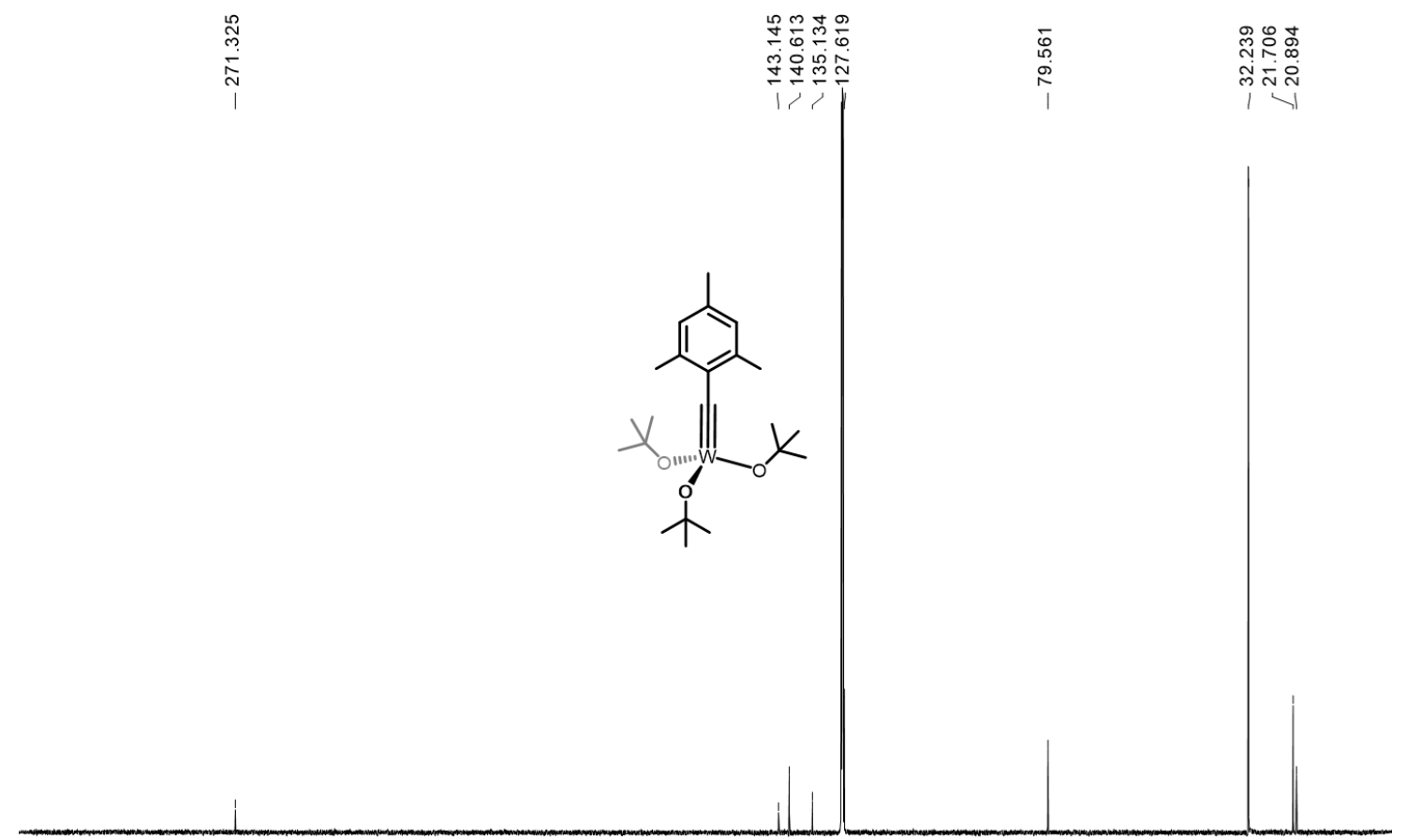

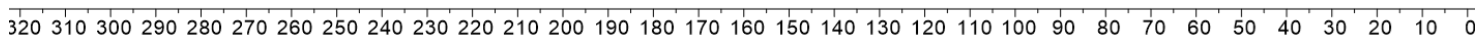

Figure S10. ${ }^{13} \mathrm{C}$ NMR $\left(125 \mathrm{MHz}, \mathrm{C}_{6} \mathrm{D}_{6}, 25{ }^{\circ} \mathrm{C}\right)$ spectrum of Pre2. 

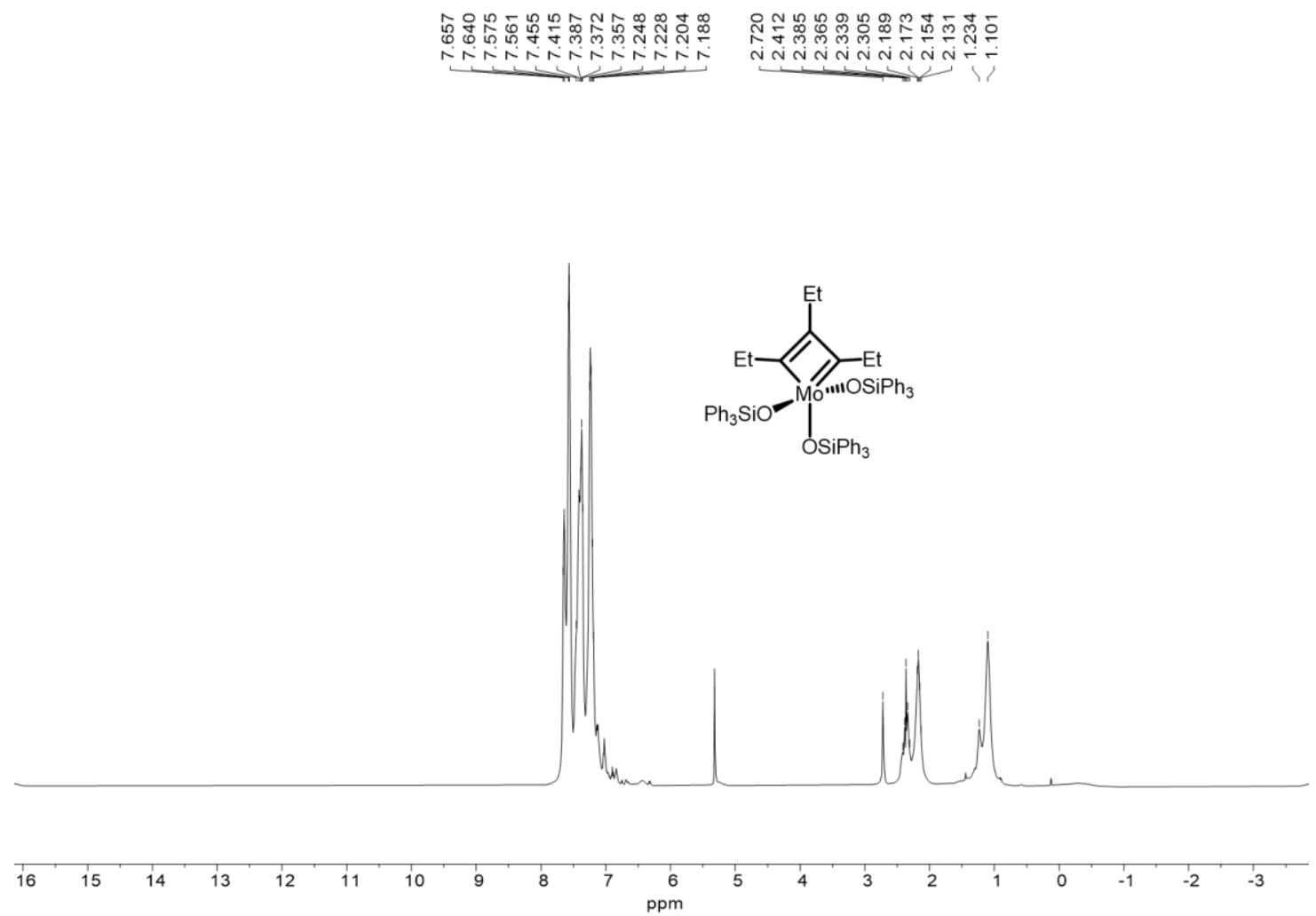

Figure S11. ${ }^{1} \mathrm{H}$ NMR $\left(500 \mathrm{MHz}, \mathrm{CD}_{2} \mathrm{Cl}_{2}, 25{ }^{\circ} \mathrm{C}\right)$ spectrum of MCBD1. 


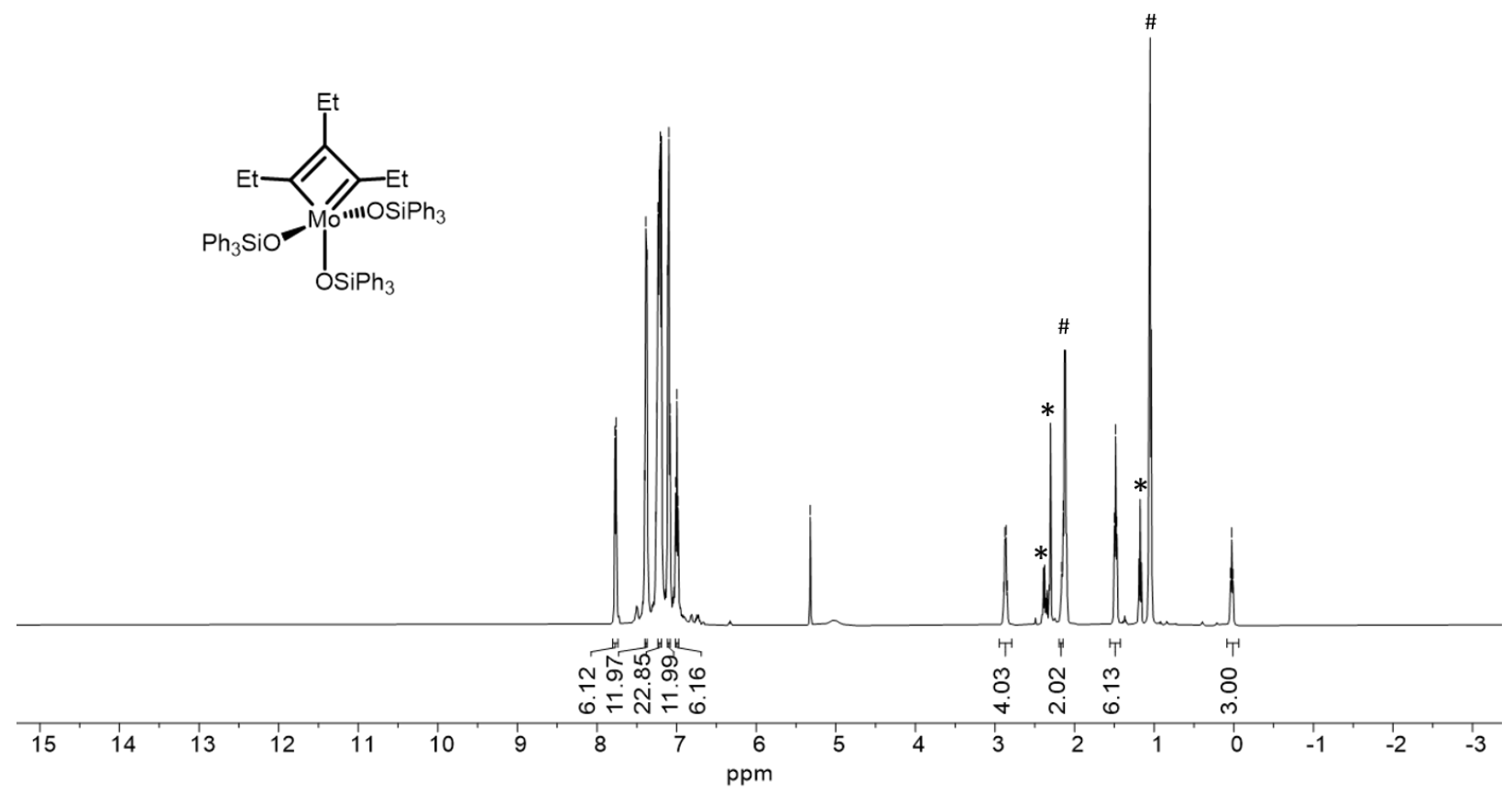

Figure S12. ${ }^{1} \mathrm{H}$ NMR $\left(500 \mathrm{MHz}, \mathrm{CD}_{2} \mathrm{Cl}_{2},-70{ }^{\circ} \mathrm{C}\right)$ spectrum of MCBD1. * Denotes 4-tolyl-1butyne byproduct and \# denotes 3-hexyne. 

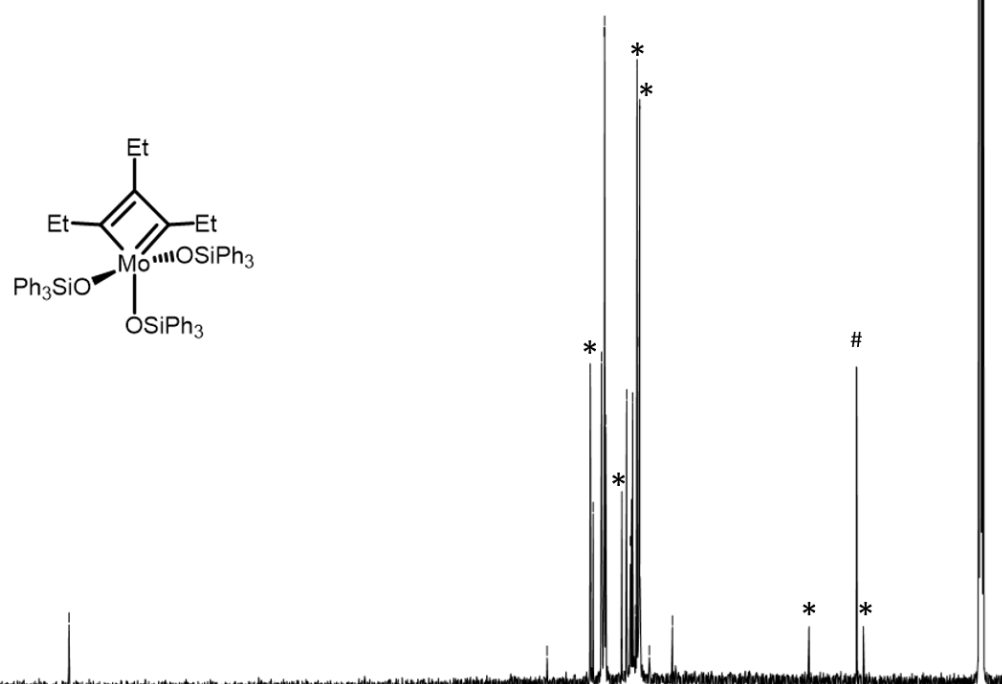

$34033032031030029028027026025024023022021020019018017016015014013012011010090 \quad 80 \quad 70 \quad 60 \quad 50 \quad 40 \quad 30 \quad 20 \quad 10 \quad 0$

Figure S13. ${ }^{13} \mathrm{C}$ NMR $\left(125 \mathrm{MHz}, \mathrm{CD}_{2} \mathrm{Cl}_{2},-70{ }^{\circ} \mathrm{C}\right)$ spectrum of MCBD1. * Denotes 4-tolyl-1butyne byproduct and \# denotes 3-hexyne. 


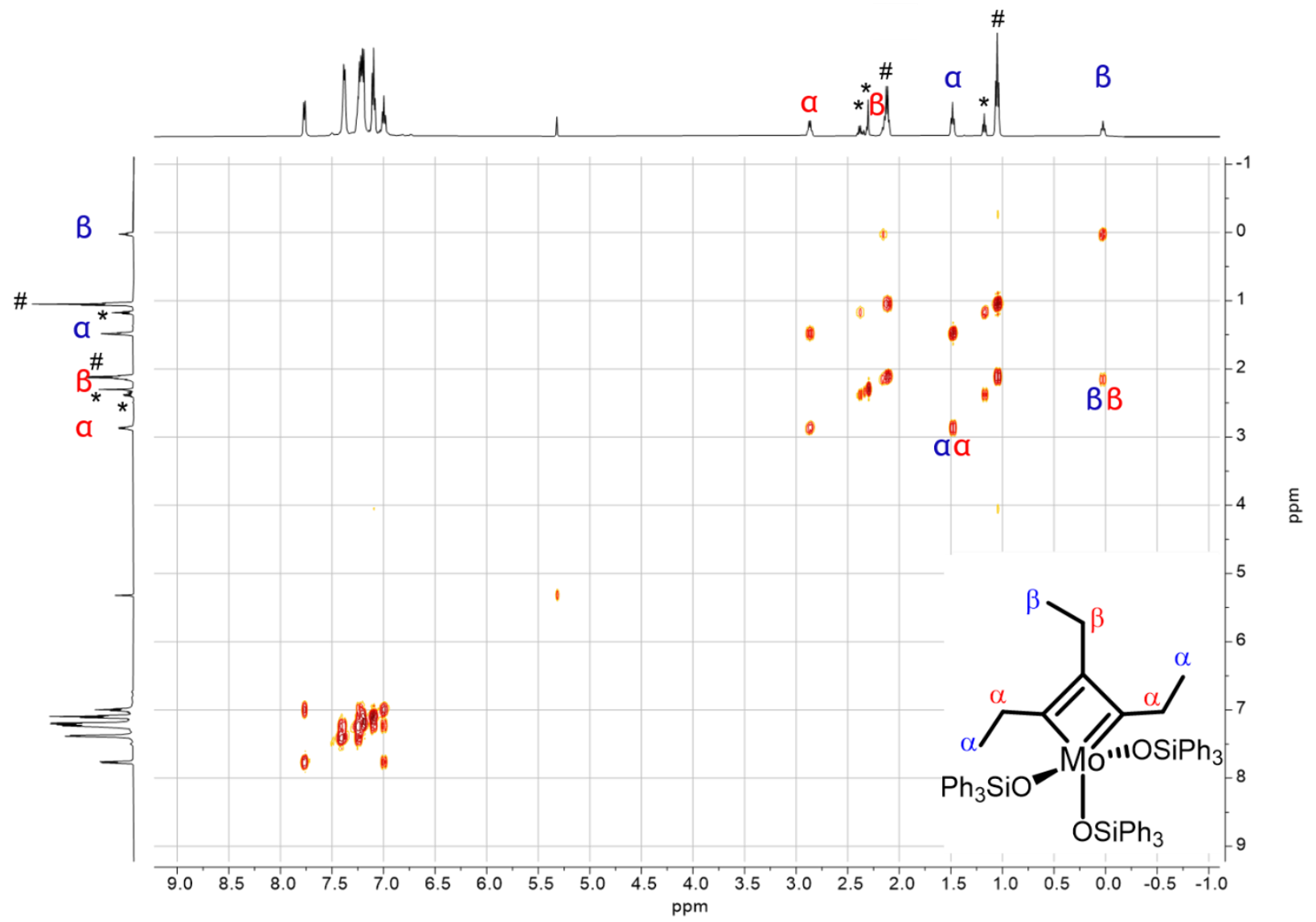

Figure S14. COSY NMR (500 MHz, $\left.\mathrm{CD}_{2} \mathrm{Cl}_{2},-70{ }^{\circ} \mathrm{C}\right)$ spectrum of MCBD1. * Denotes 4-tolyl-1butyne and \# denotes 3-hexyne. 


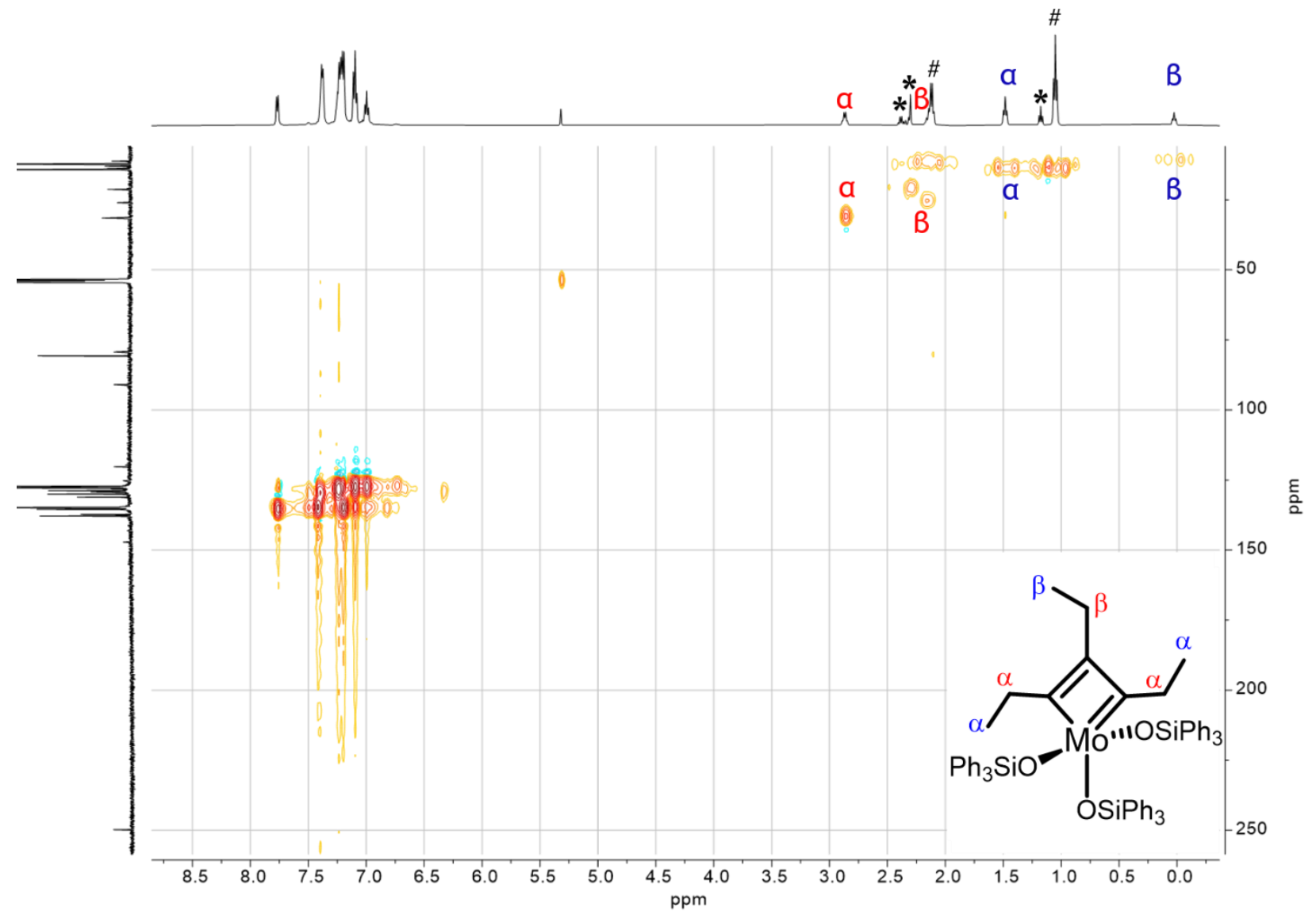

Figure S15. HSQC NMR (400 MHz, $\mathrm{CD}_{2} \mathrm{Cl}_{2},-70{ }^{\circ} \mathrm{C}$ ) spectrum of MCBD1. * Denotes 4-tolyl-1butyne byproduct and \# denotes 3-hexyne. 


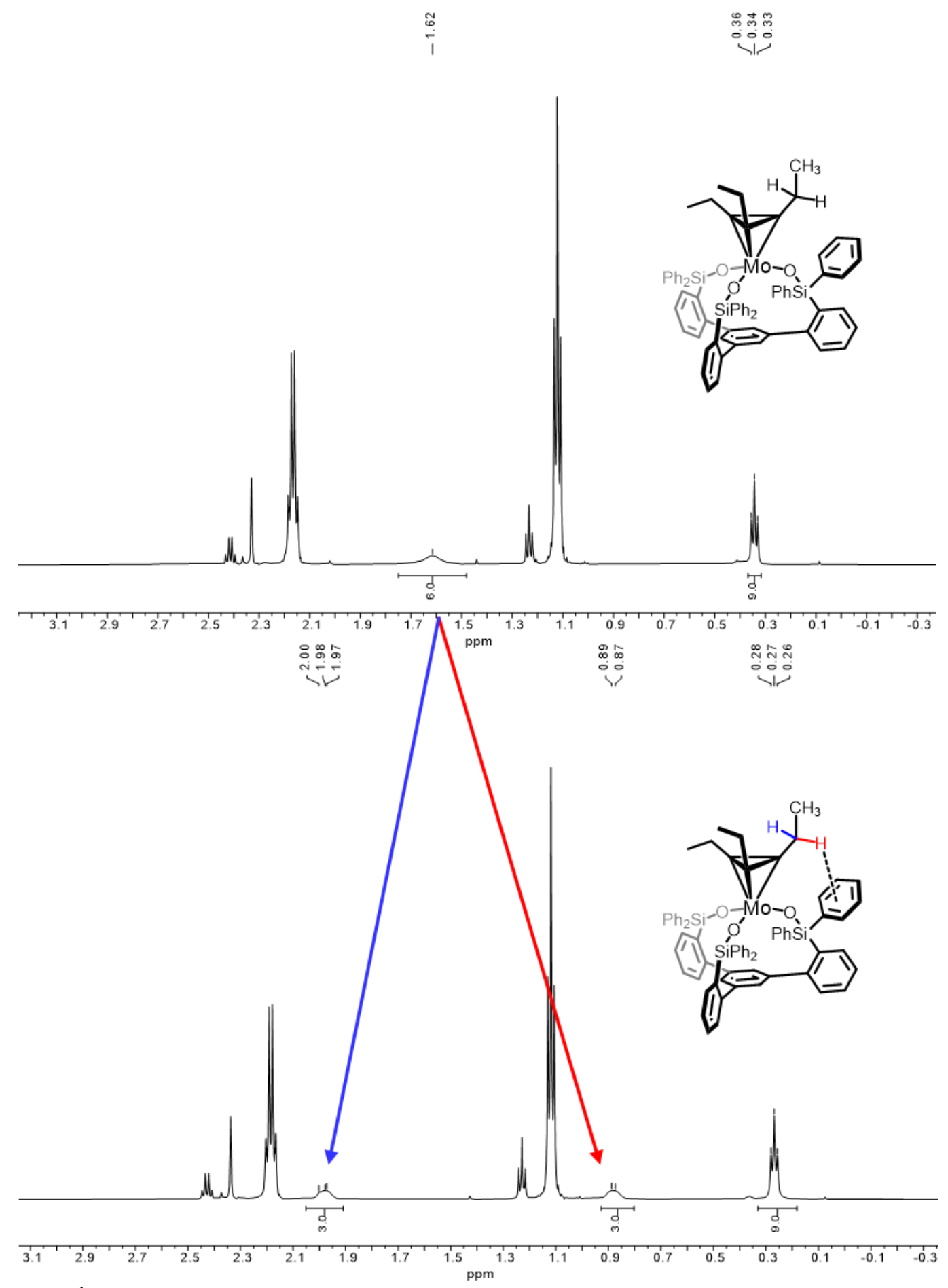

Figure S16. ${ }^{1} \mathrm{H}$ NMR $\left(\mathrm{CDCl}_{3}\right), 13{ }^{\circ} \mathrm{C}$ (top) and $-60{ }^{\circ} \mathrm{C}$ (bottom) spectra of $\mathbf{M t}_{\mathbf{d}} \mathbf{1}$ showing the splitting of the diastereotopic methylene protons with the $\mathrm{C}-\mathrm{H}^{\cdots} \pi$ interactions strongly shielding one set. 


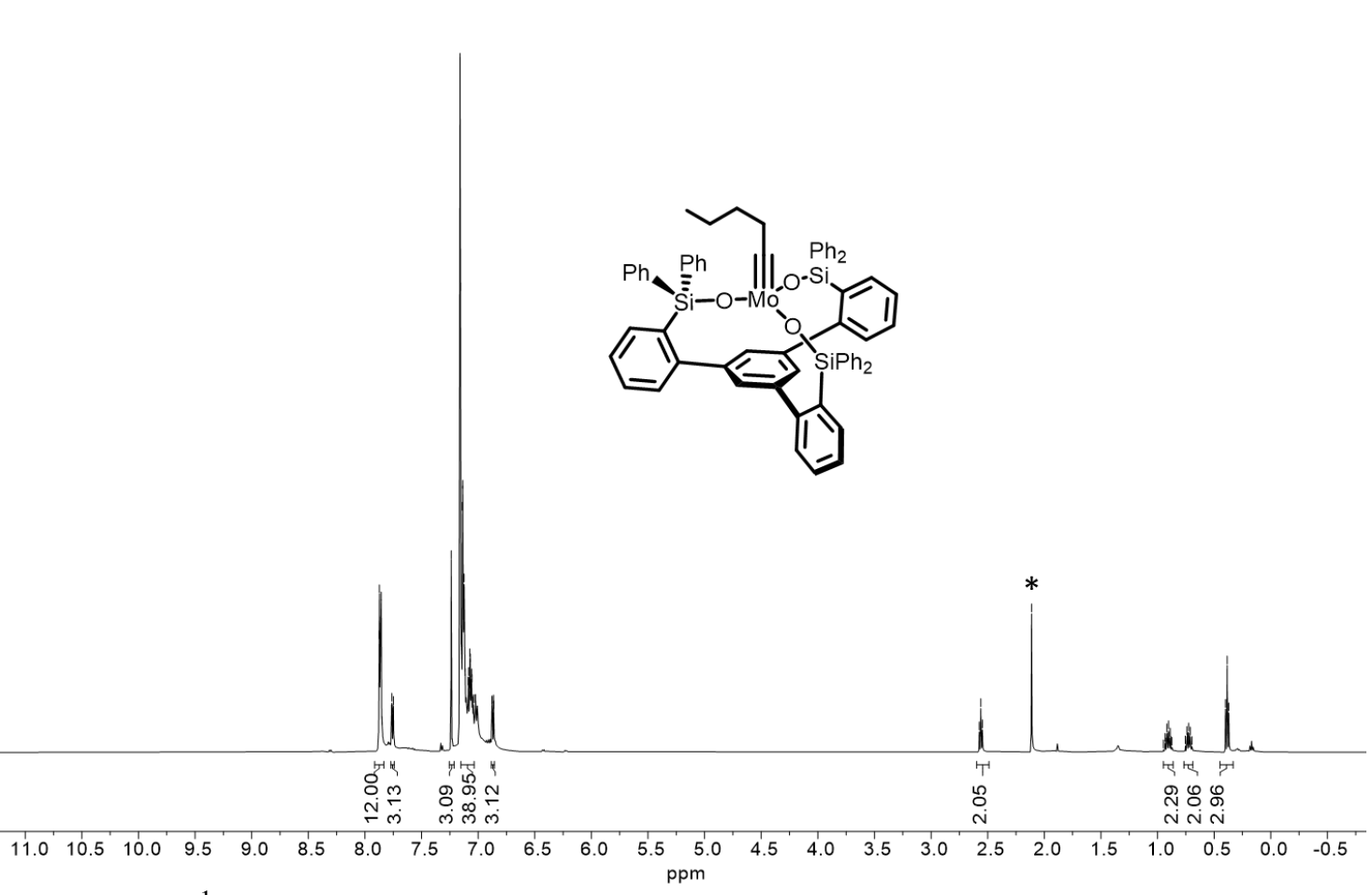

Figure S17. ${ }^{1} \mathrm{H}$ NMR $\left(500 \mathrm{MHz}, \mathrm{C}_{6} \mathrm{D}_{6}, 25^{\circ} \mathrm{C}\right)$ spectrum of Cat7. * Denotes toluene impurity

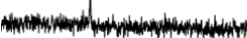

$328 \cdot 326-324-322-320-318$

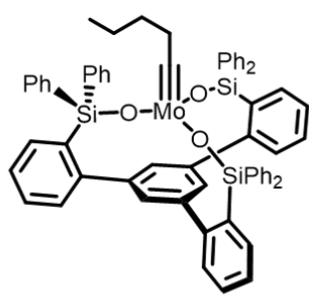

$340330320310300290280270260250240230220210200190180170160150140130120110100908070 \quad 6050 \quad 40 \quad 3020 \quad 10$ ppm

Figure S18. ${ }^{13} \mathrm{C}$ NMR $\left(125 \mathrm{MHz}, \mathrm{C}_{6} \mathrm{D}_{6}, 25{ }^{\circ} \mathrm{C}\right)$ spectrum of Cat7. * Denotes toluene impurity 


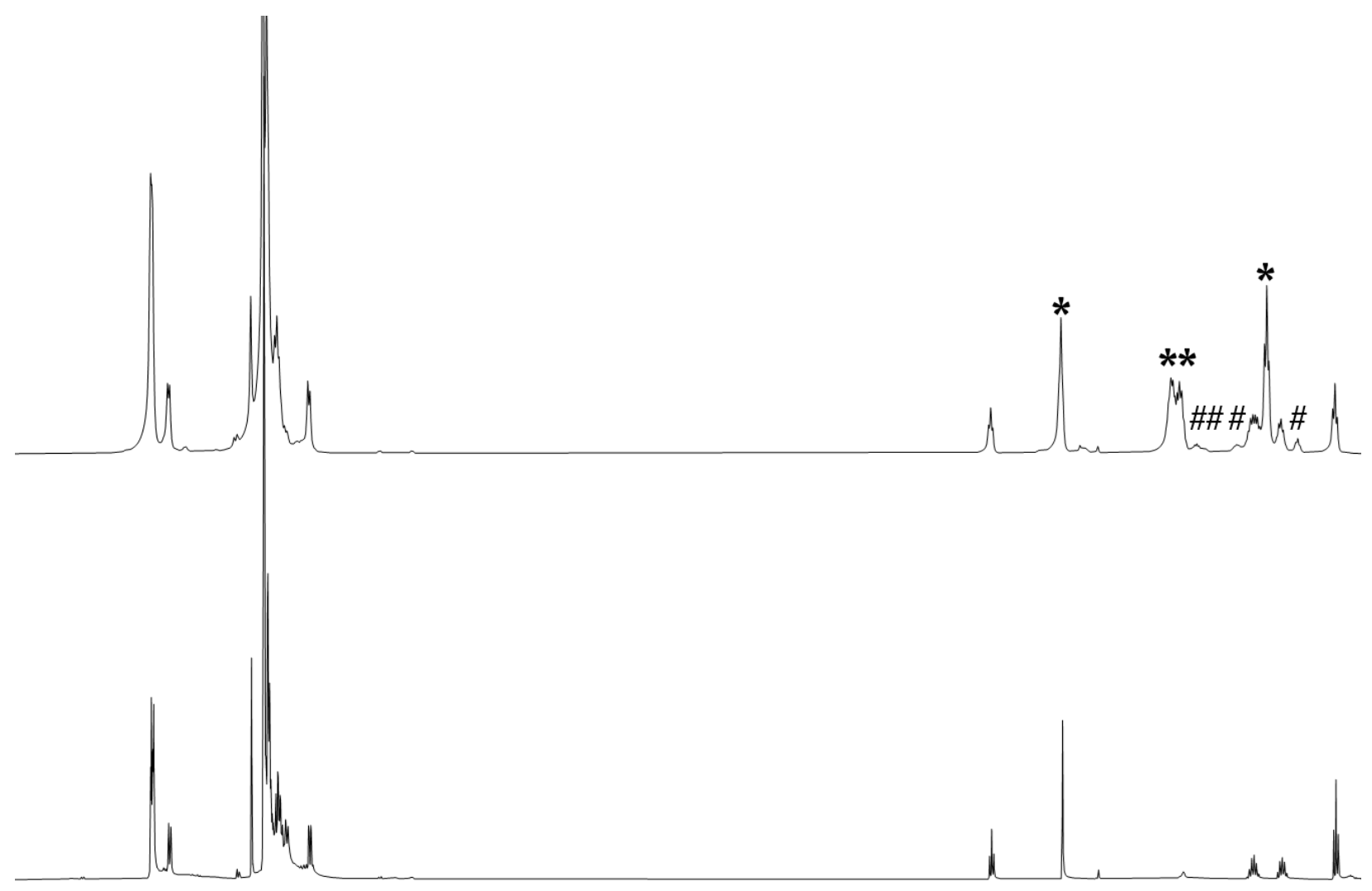

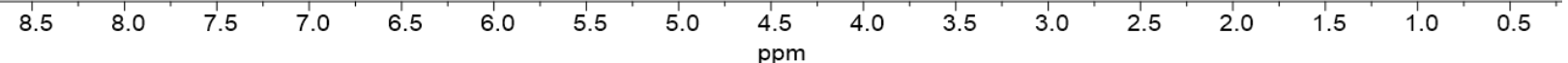

Figure S19. ${ }^{1} \mathrm{H}$ NMR $\left(500 \mathrm{MHz}, \mathrm{C}_{6} \mathrm{D}_{6}, 25^{\circ} \mathrm{C}\right)$, of pure Cat7 (bottom) and after adding 10 eq of 5-decyne (Top). Mtd $\mathbf{2}$ is denoted by \# and free 5-decyne is denoted by *. 


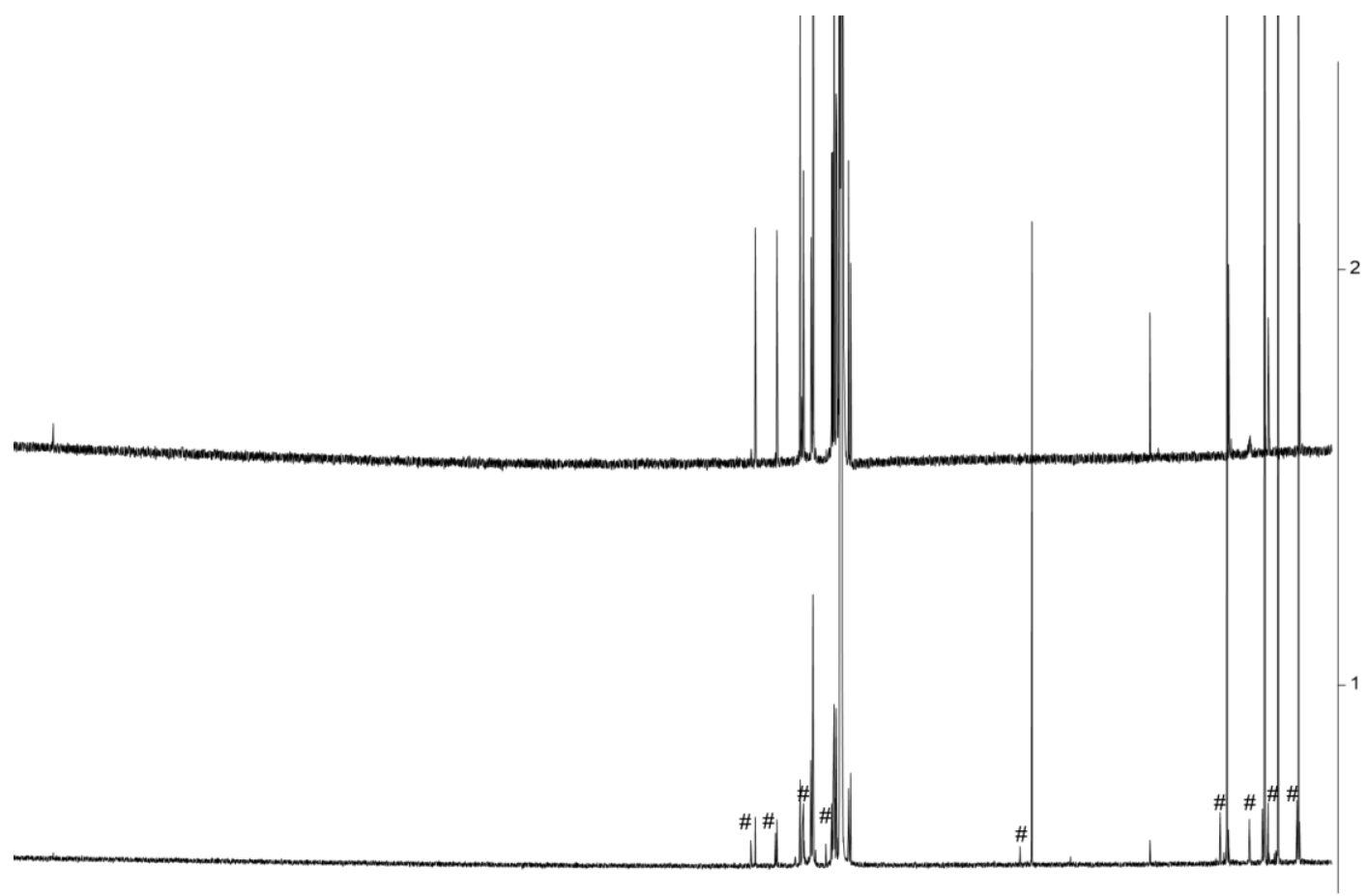

$33032031030029028027026025024023022021020019018017016015014013012011010090 \quad 80 \quad 70 \quad 60 \quad 50 \quad 40 \quad 30 \quad 20 \quad 10$ $\mathrm{ppm}$

Figure S20. ${ }^{13} \mathrm{C}$ NMR (125 MHz, $\mathrm{C}_{6} \mathrm{D}_{6}, 25{ }^{\circ} \mathrm{C}$ ), of pure Cat7 (top) and after adding 10 eq of 5decyne (bottom). $\mathbf{M t}_{\mathbf{d}} \mathbf{2}$ is denoted with \#, the $C_{3} \mathrm{Bu}_{3}$ resonance is identified at $83.4 \mathrm{ppm}$. 


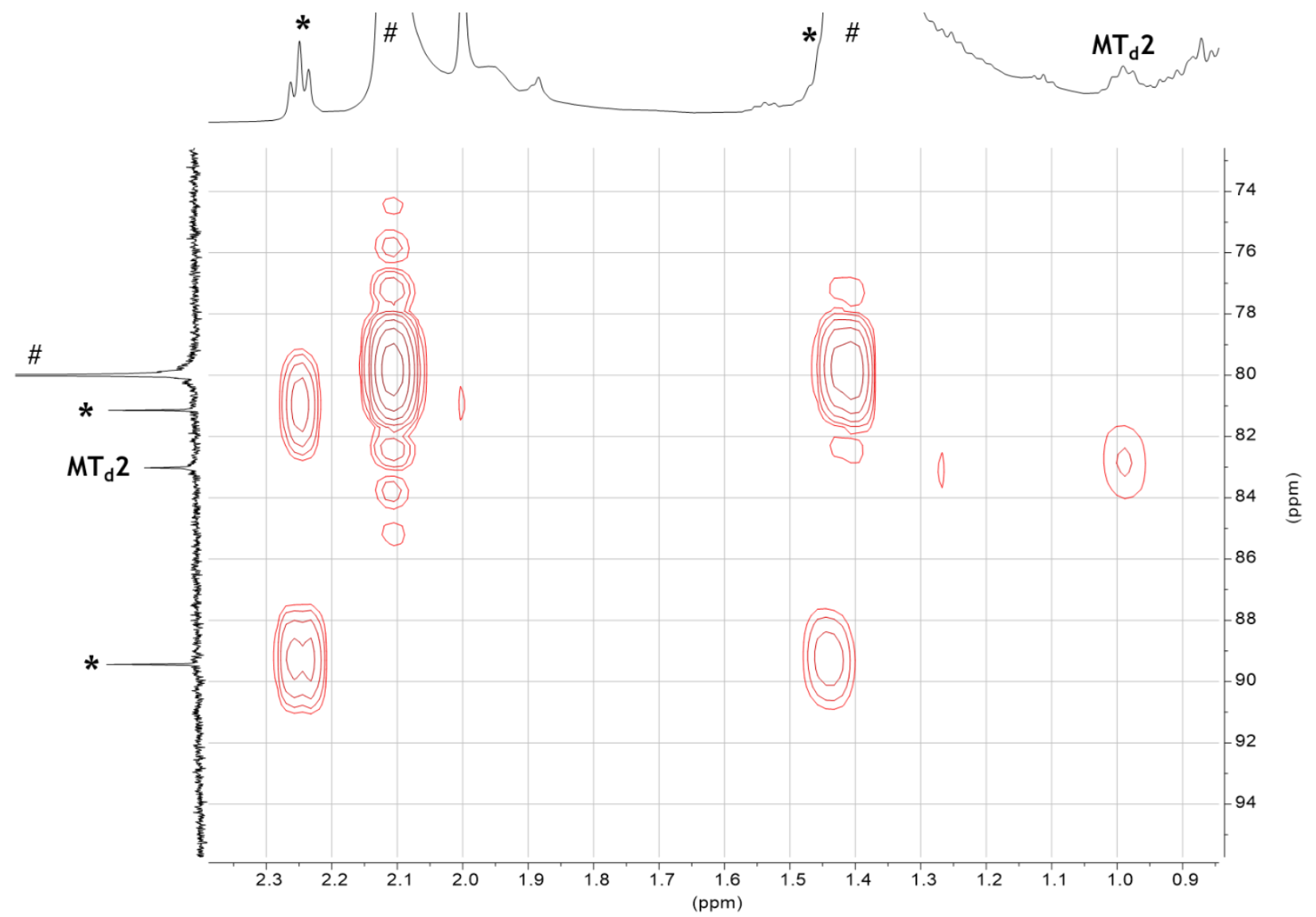

Figure S21. Long-range coupling ${ }^{1} \mathrm{H}_{-}{ }^{13} \mathrm{C}$ gHMBC NMR (500 MHz, C6D6, $25{ }^{\circ} \mathrm{C}$ ) spectrum of $\mathbf{M T}_{\mathrm{d}} \mathbf{2}$ generated in-situ by the addition of excess 5-decyne (\#) to a solution of Cat3. 4-tolyl-1-hexyne impurity is denoted by *. 


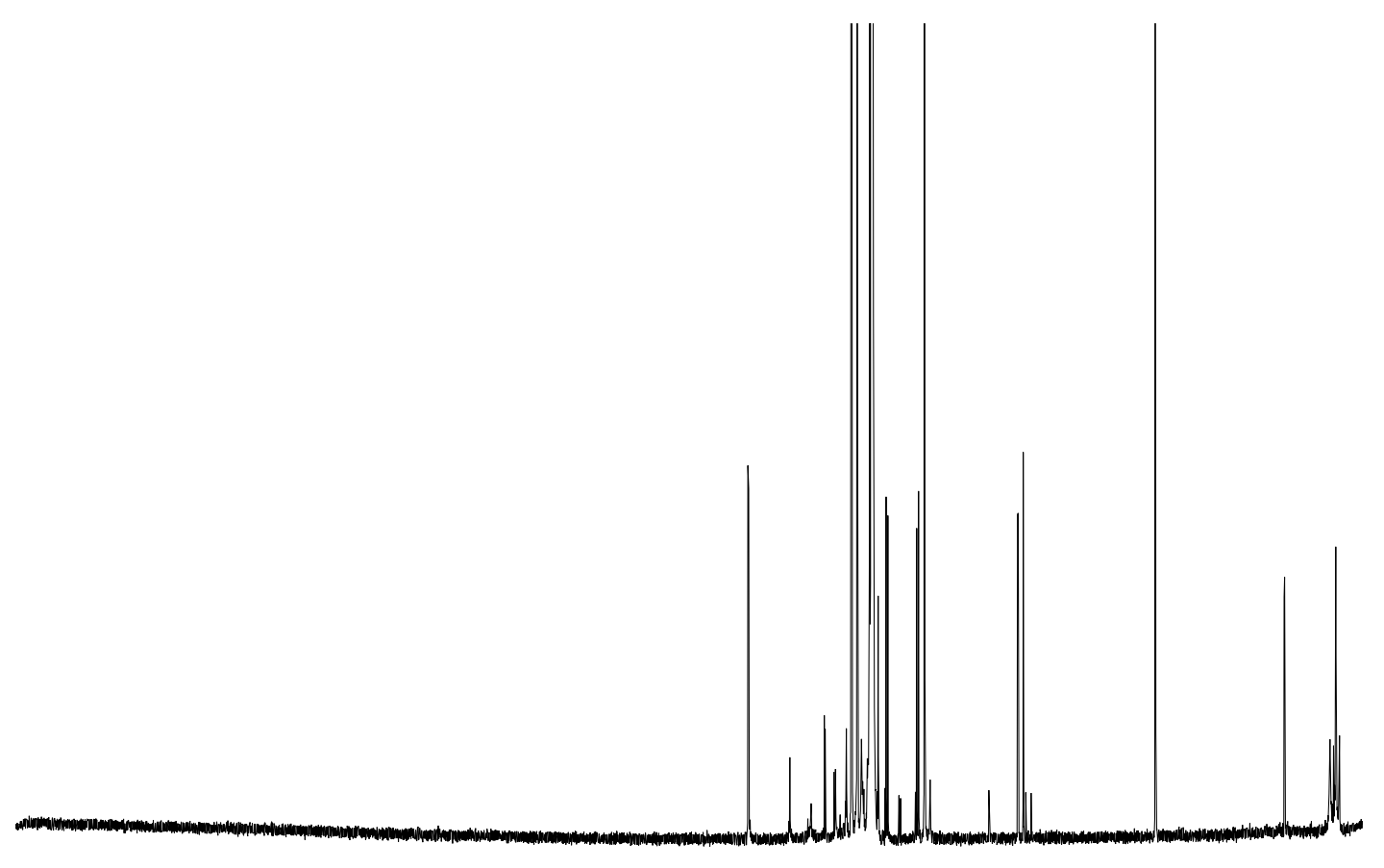

$34033032031030029028027026025024023022021020019018017016015014013012011010090 \quad 8070 \quad 60 \quad 50 \quad 40 \quad 30 \quad 20 \quad 10$

Figure S22. ${ }^{13} \mathrm{C}$ NMR $\left(\mathrm{C}_{6} \mathrm{D}_{6}\right)$ of the reaction of Cat5 with 6 eq. of 1-methoxy-4(phenylethynl)benzene. The absence of resonances between 220-280 suggests that an all aryl MCBD is not the resting state.

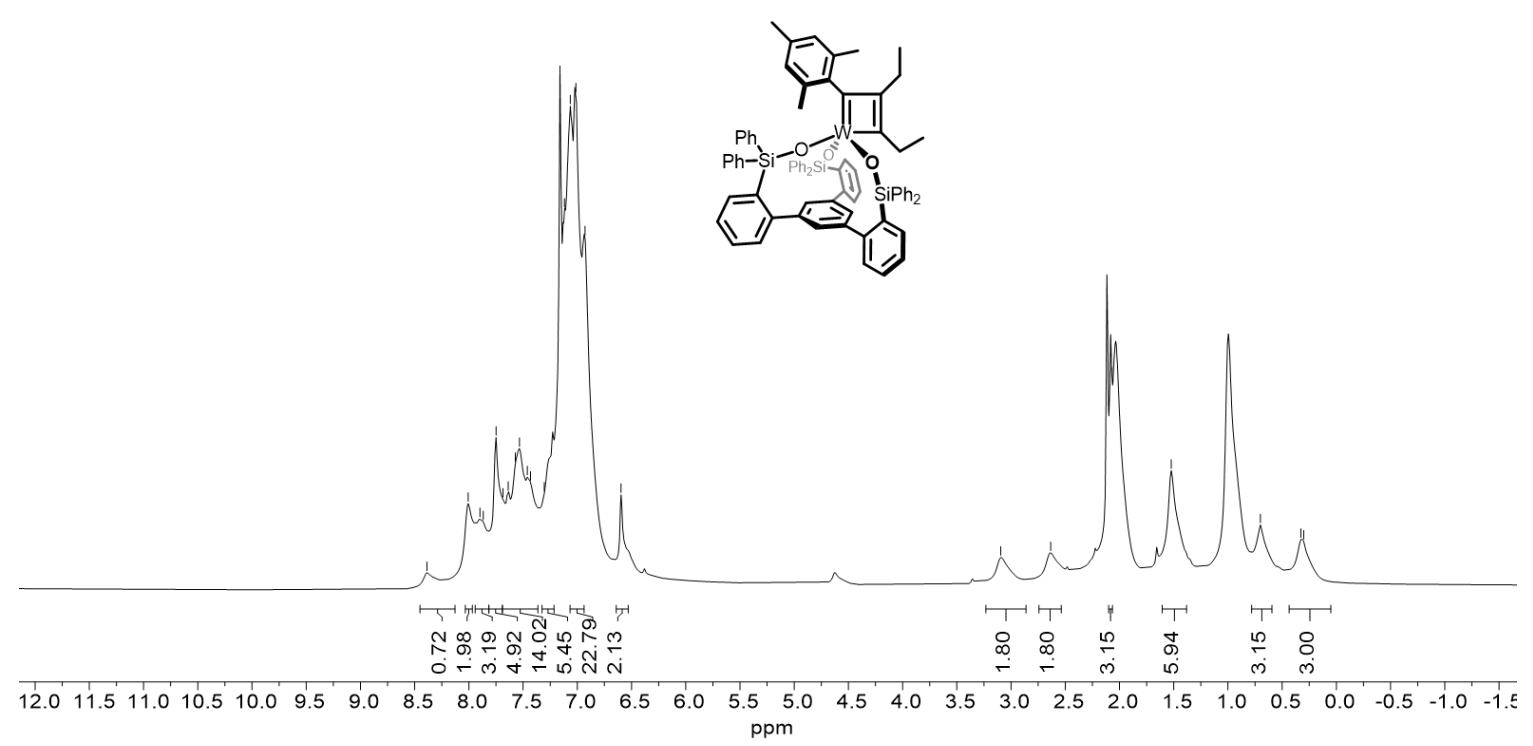

Figure S23 ${ }^{1} \mathrm{H}$ NMR $\left(500 \mathrm{MHz}, \mathrm{C}_{6} \mathrm{D}_{6}, 25{ }^{\circ} \mathrm{C}\right)$ spectrum of MCBD2. 


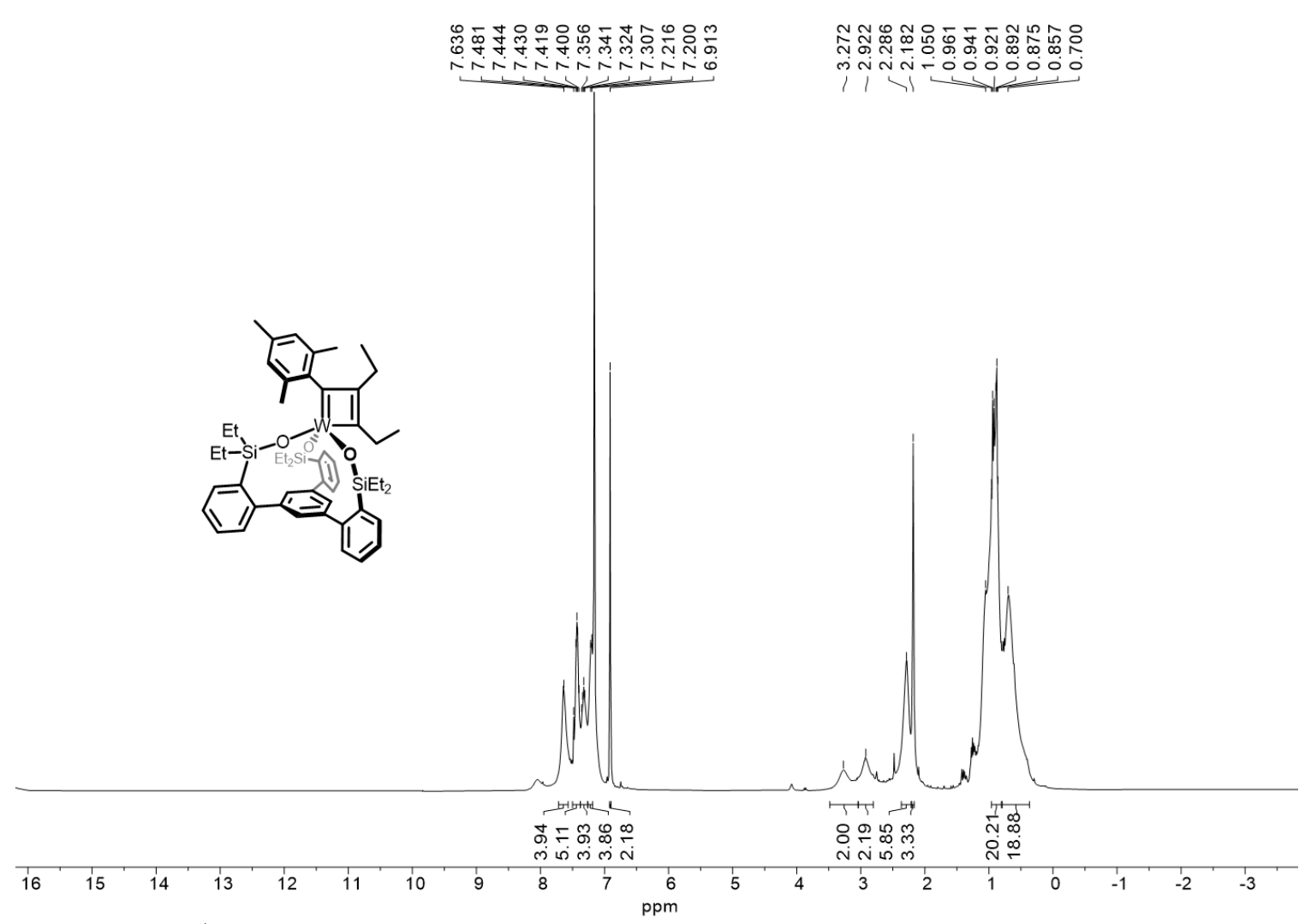

Figure S24. ${ }^{1} \mathrm{H}$ NMR $\left(400 \mathrm{MHz}, \mathrm{C}_{6} \mathrm{D}_{6}, 25^{\circ} \mathrm{C}\right)$ spectrum of MCBD3.

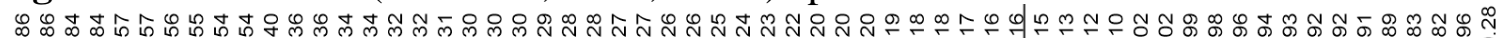

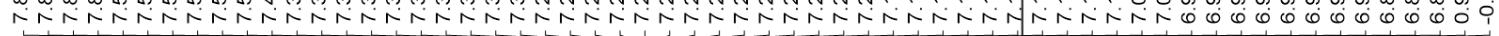

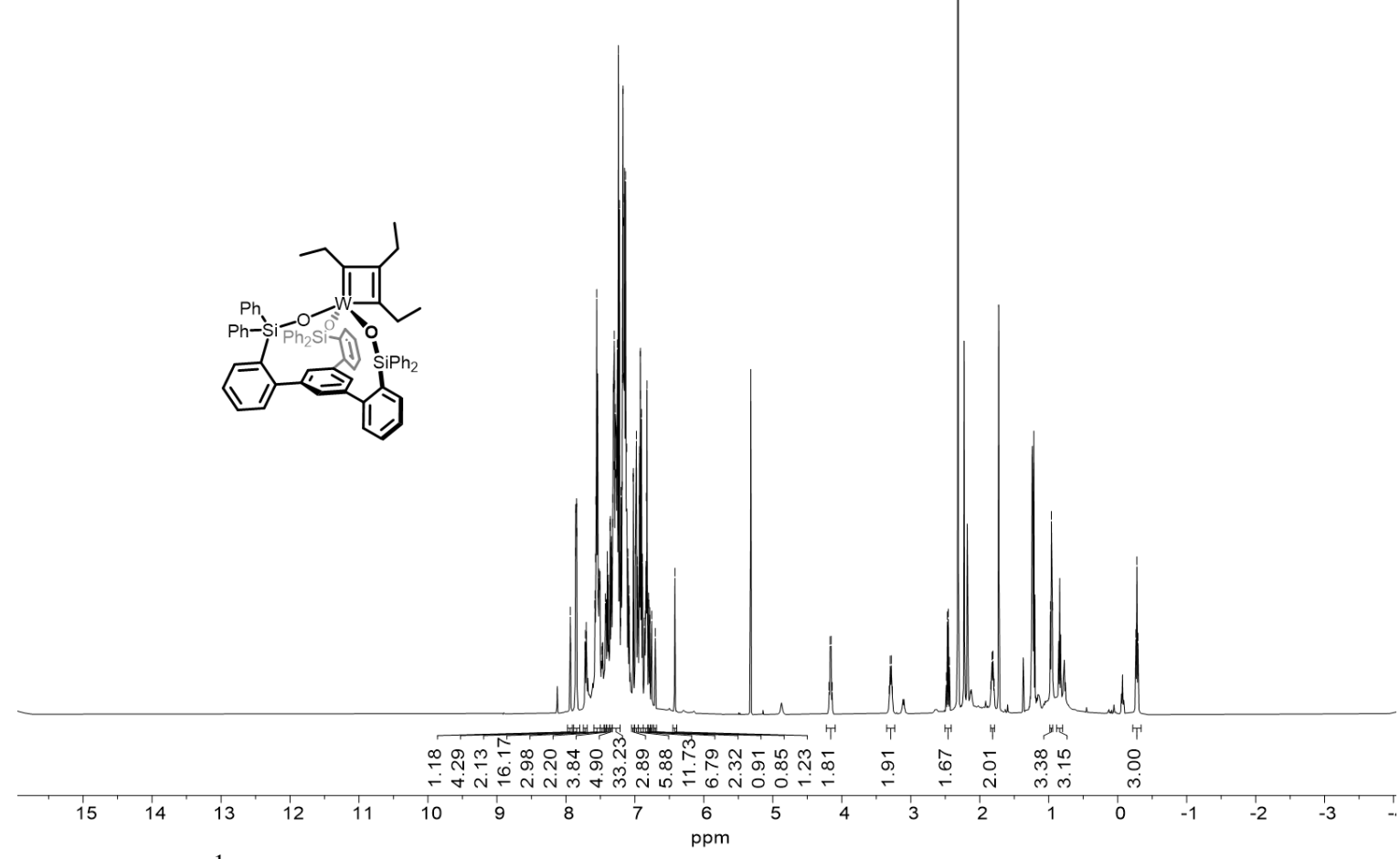

Figure S25. ${ }^{1} \mathrm{H} \mathrm{NMR}\left(500 \mathrm{MHz}, \mathrm{CD}_{2} \mathrm{Cl}_{2},-70{ }^{\circ} \mathrm{C}\right)$ spectrum of MCBD4. 


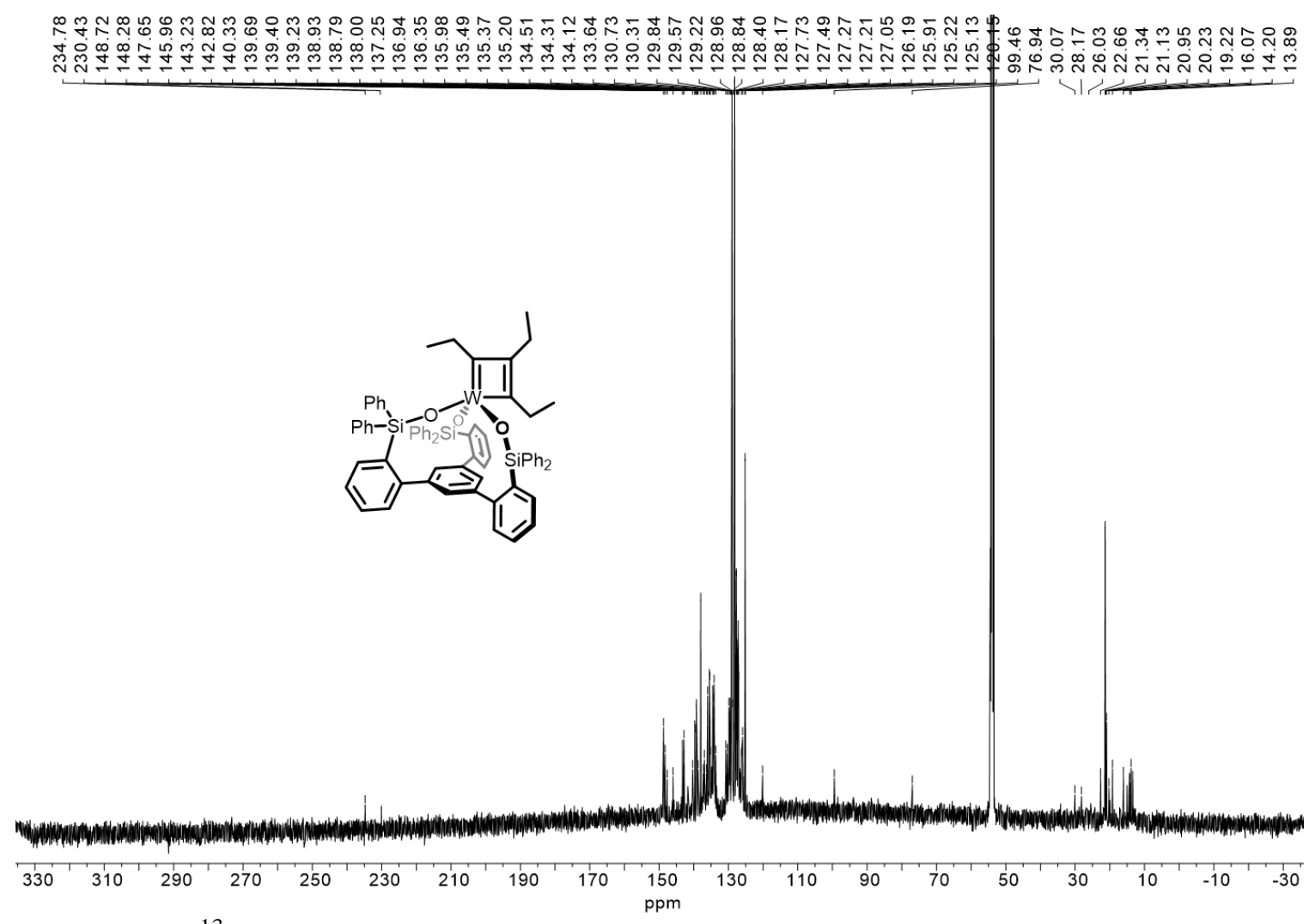

Figure S26. ${ }^{13} \mathrm{C}$ NMR $\left(125 \mathrm{MHz}, \mathrm{CD}_{2} \mathrm{Cl}_{2},-70{ }^{\circ} \mathrm{C}\right)$ spectrum of MCBD4. 


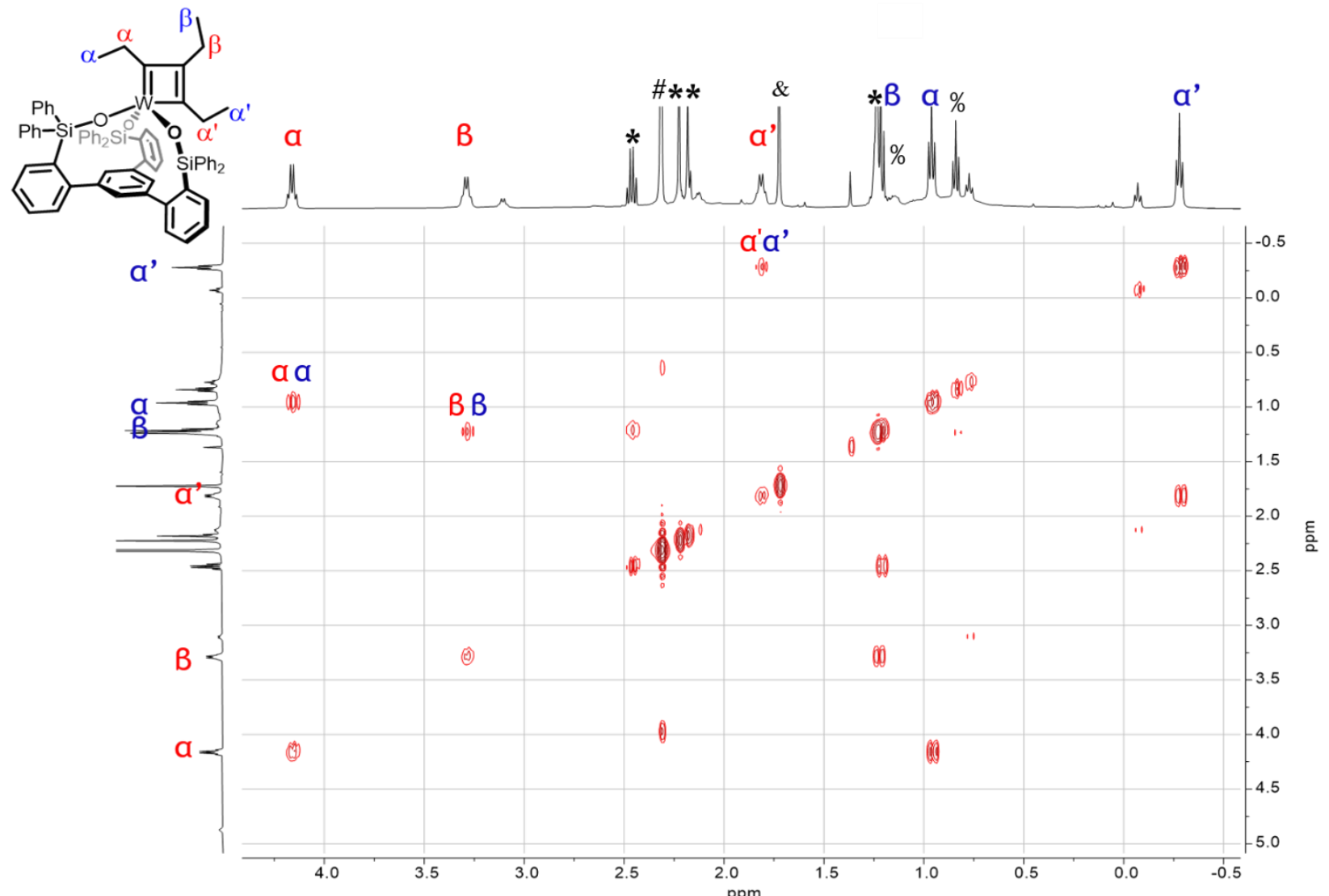

Figure S27. ${ }^{1} \mathrm{H}-{ }^{1} \mathrm{H}$ Through-bond coupling gCOSY NMR $\left(500 \mathrm{MHz}, \mathrm{CD}_{2} \mathrm{Cl}_{2},-70{ }^{\circ} \mathrm{C}\right)$ spectrum of MCBD4. * Denotes mesityl-1-butyne byproduct, \# denotes toluene impurity, \% denotes pentane impurity and \& denotes a minor impurity which forms overtime within 3-hexyne. 


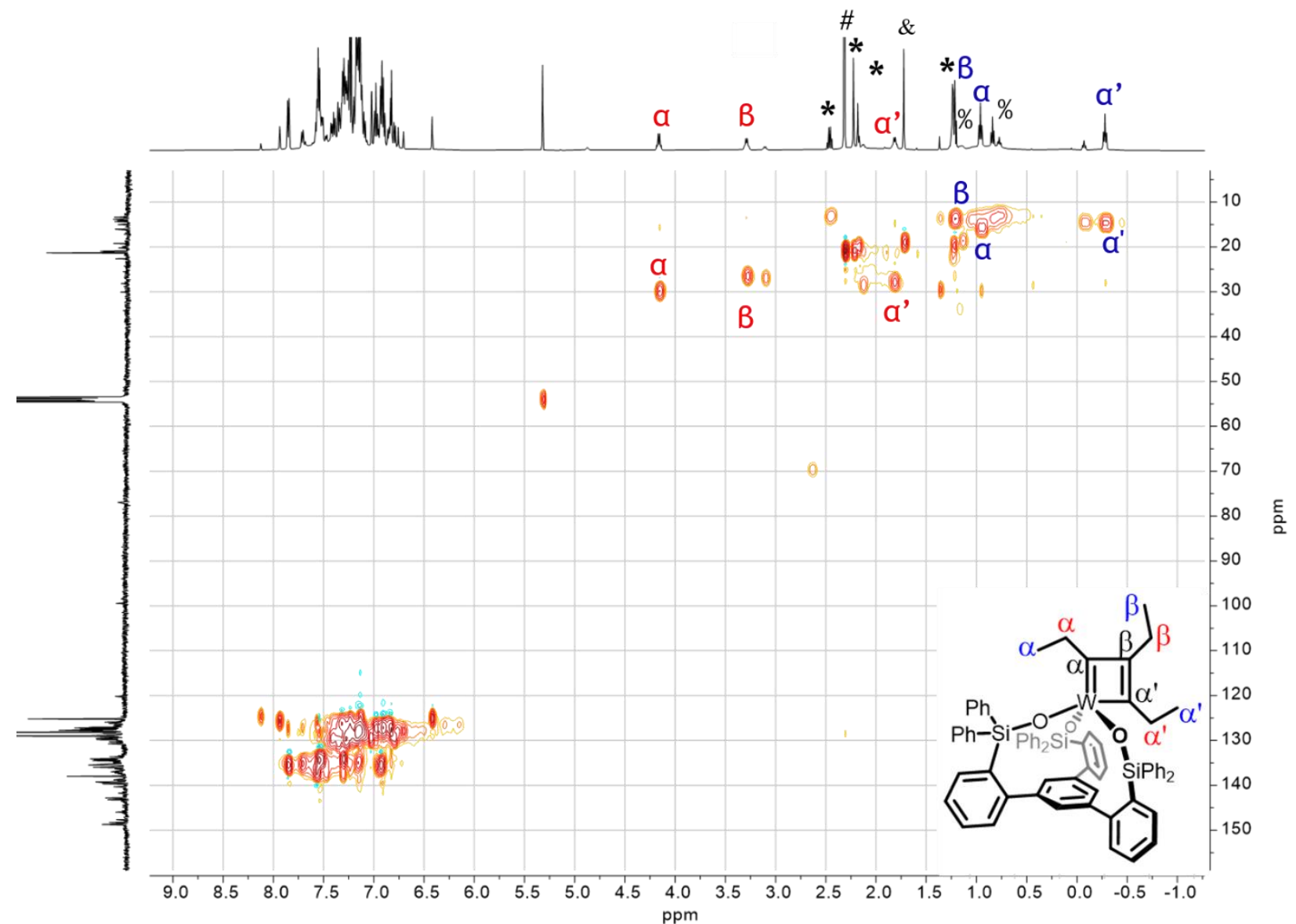

Figure S28. One-bond coupling ${ }^{1} \mathrm{H}^{-13} \mathrm{C}$ gHSQC NMR $\left(500 \mathrm{MHz}, \mathrm{CD}_{2} \mathrm{Cl}_{2},-70{ }^{\circ} \mathrm{C}\right)$ spectrum of MCBD4. * Denotes mesityl-1-butyne byproduct, \# denotes toluene impurity, \% denotes pentane impurity and \& denotes a minor impurity which forms overtime within 3-hexyne. 


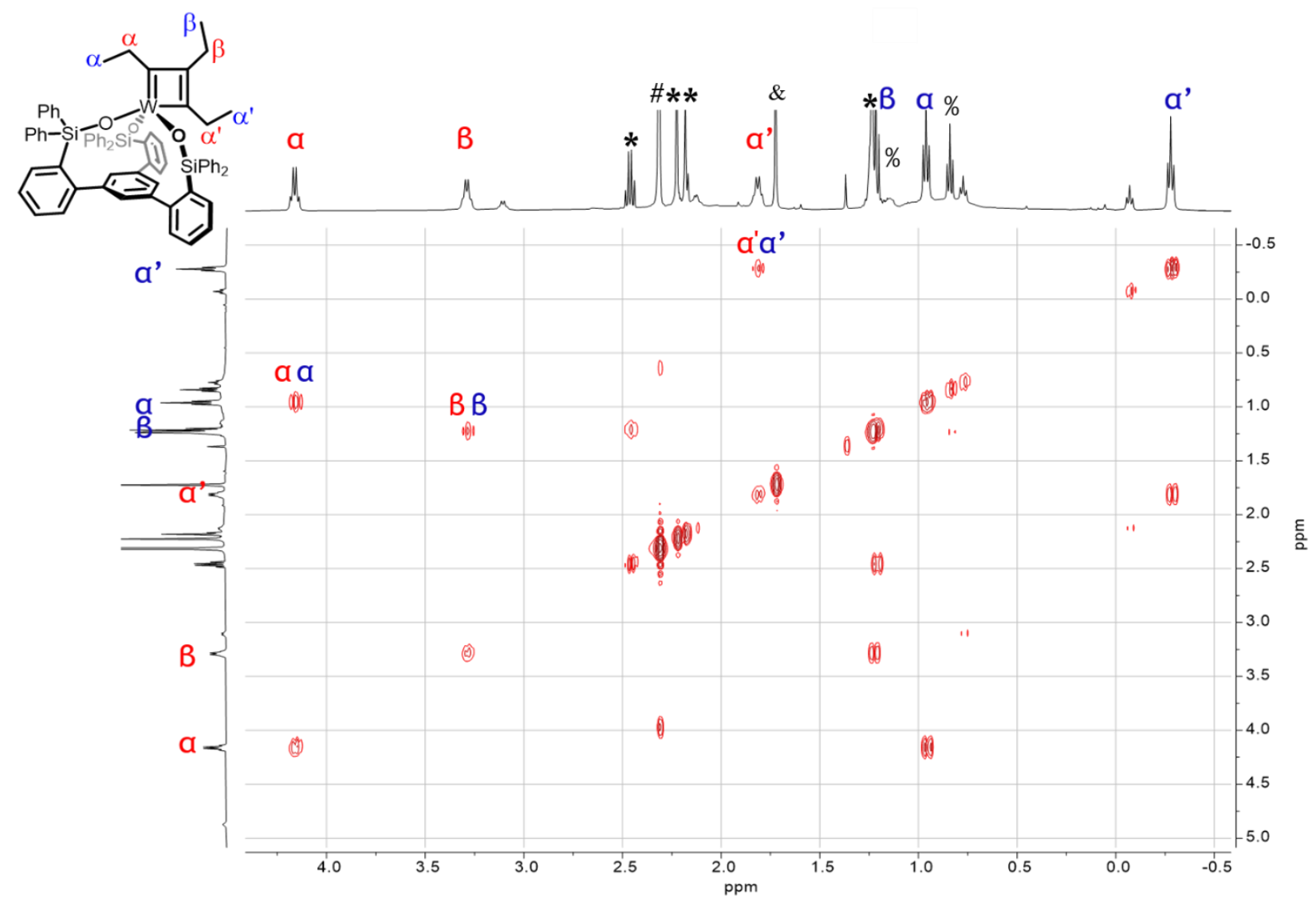

Figure S29. Long-range coupling ${ }^{1} \mathrm{H}-{ }^{13} \mathrm{C}$ gHMBC NMR $\left(500 \mathrm{MHz}, \mathrm{CD}_{2} \mathrm{Cl}_{2},-70{ }^{\circ} \mathrm{C}\right)$ spectrum of MCBD4. * Denotes mesityl-1-butyne byproduct, \# denotes toluene impurity, \% denotes pentane impurity and \& denotes a minor impurity which forms overtime within 3-hexyne. 


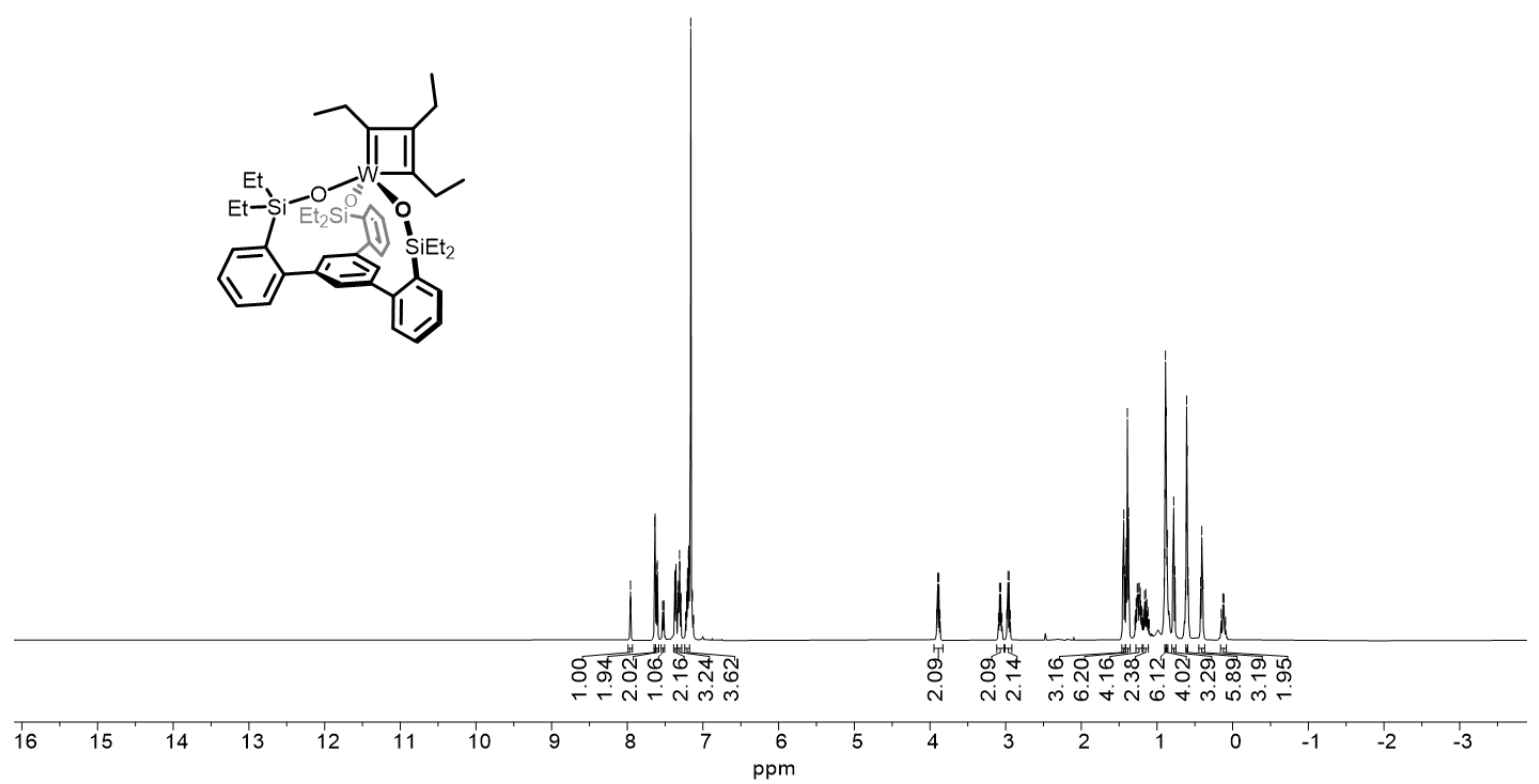

Figure S30. ${ }^{1} \mathrm{H}$ NMR $\left(500 \mathrm{MHz}, \mathrm{C}_{6} \mathrm{D}_{6}, 25^{\circ} \mathrm{C}\right)$ spectrum of MCBD5.

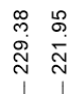

ㅇำ

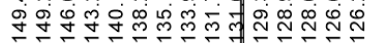

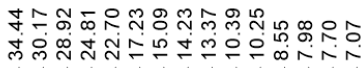
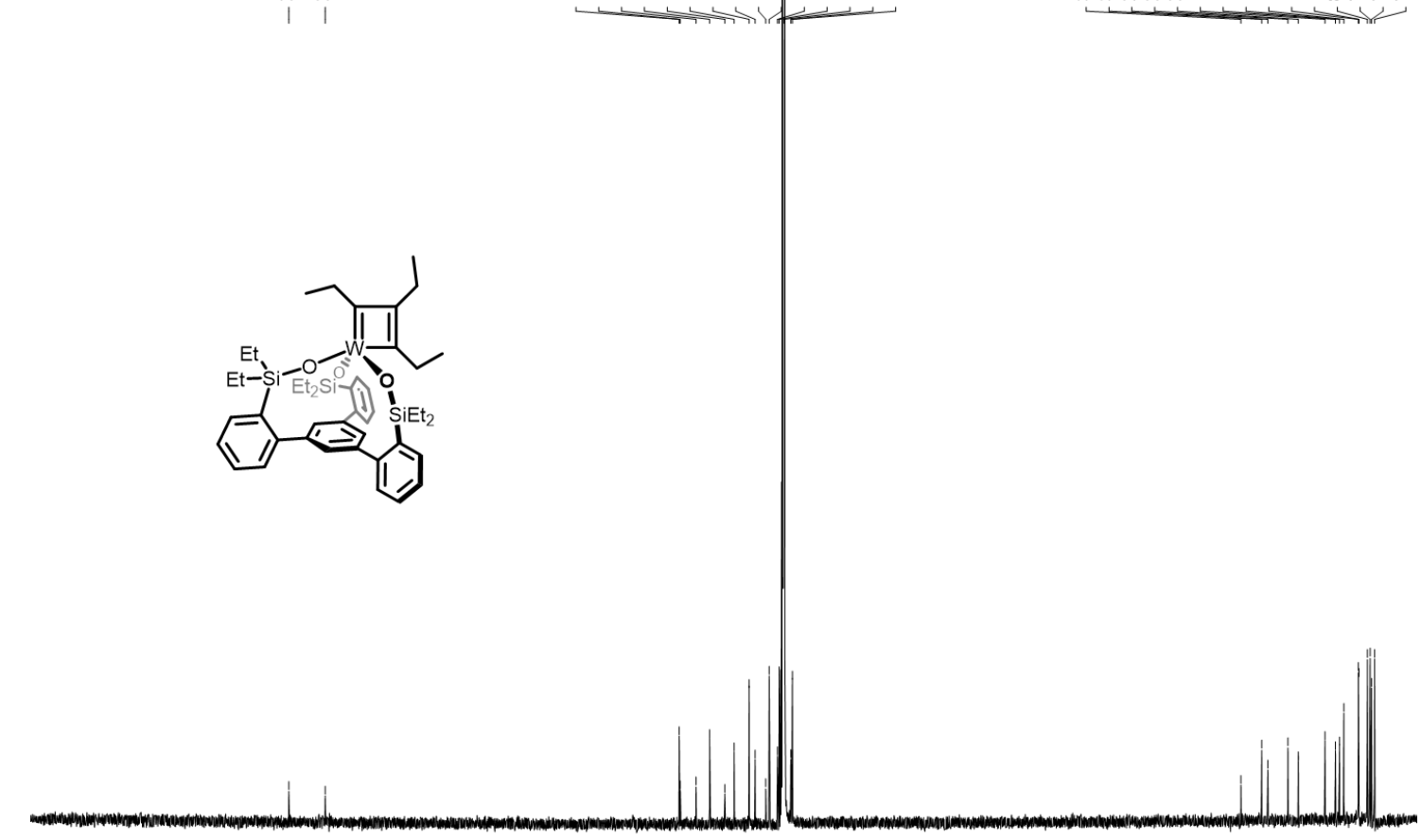

$\begin{array}{lllllllllllllllllllllllllllllllll}280 & 270 & 260 & 250 & 240 & 230 & 220 & 210 & 200 & 190 & 180 & 170 & 160 & 150 & 140 & 130 & 120 & 110 & 100 & 90 & 80 & 70 & 60 & 50 & 40 & 30 & 20 & 10 & 0\end{array}$

Figure S31. ${ }^{13} \mathrm{C}$ NMR $\left(125 \mathrm{MHz}, \mathrm{C}_{6} \mathrm{D}_{6}, 25{ }^{\circ} \mathrm{C}\right)$ spectrum of MCBD5. 


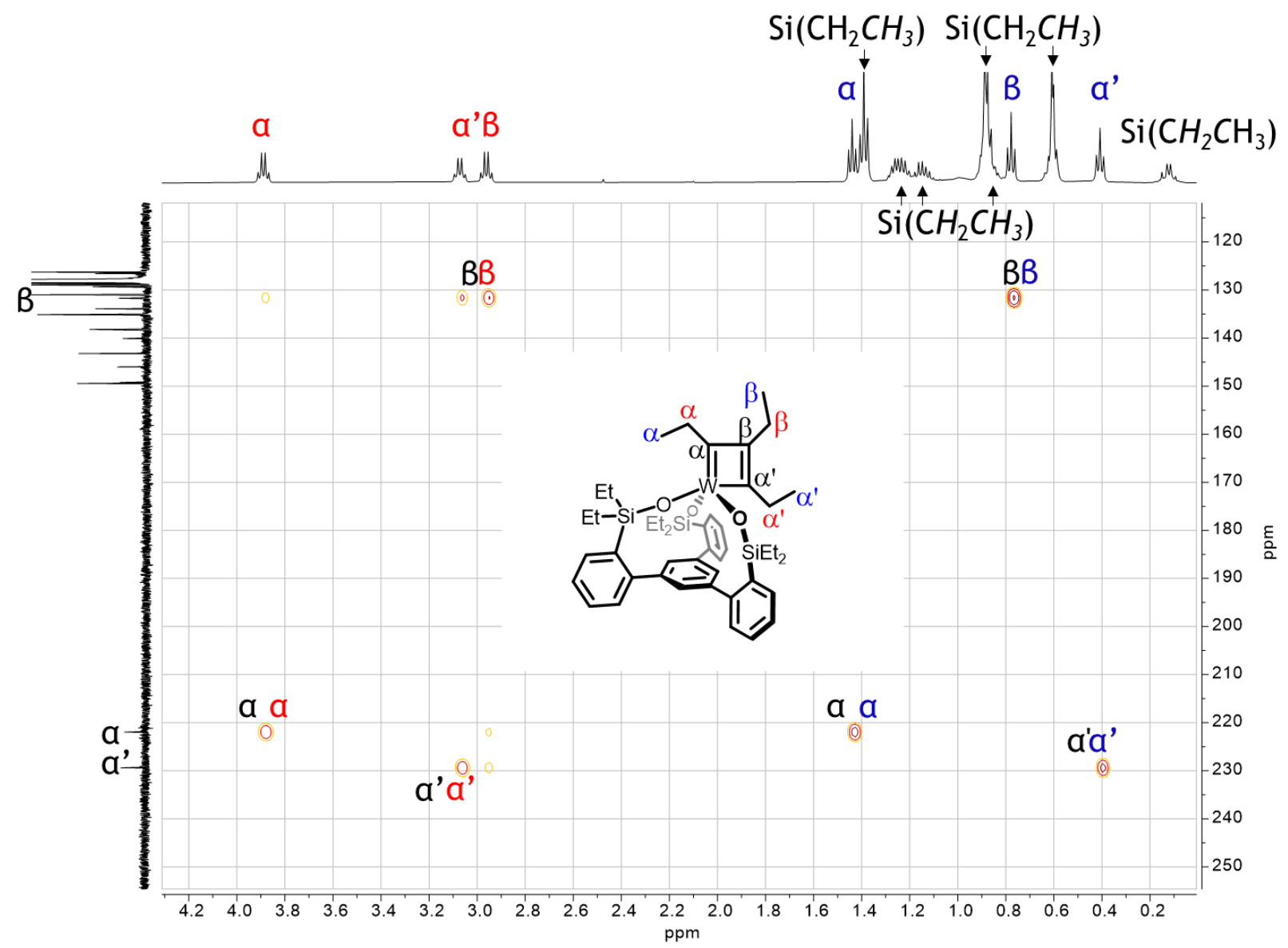

Figure S32. ${ }^{1} \mathrm{H}-{ }^{1} \mathrm{H}$ Through-bond coupling gCOSY NMR $\left(500 \mathrm{MHz}, \mathrm{C}_{6} \mathrm{D}_{6}, 25{ }^{\circ} \mathrm{C}\right)$ spectrum of MCBD5. 


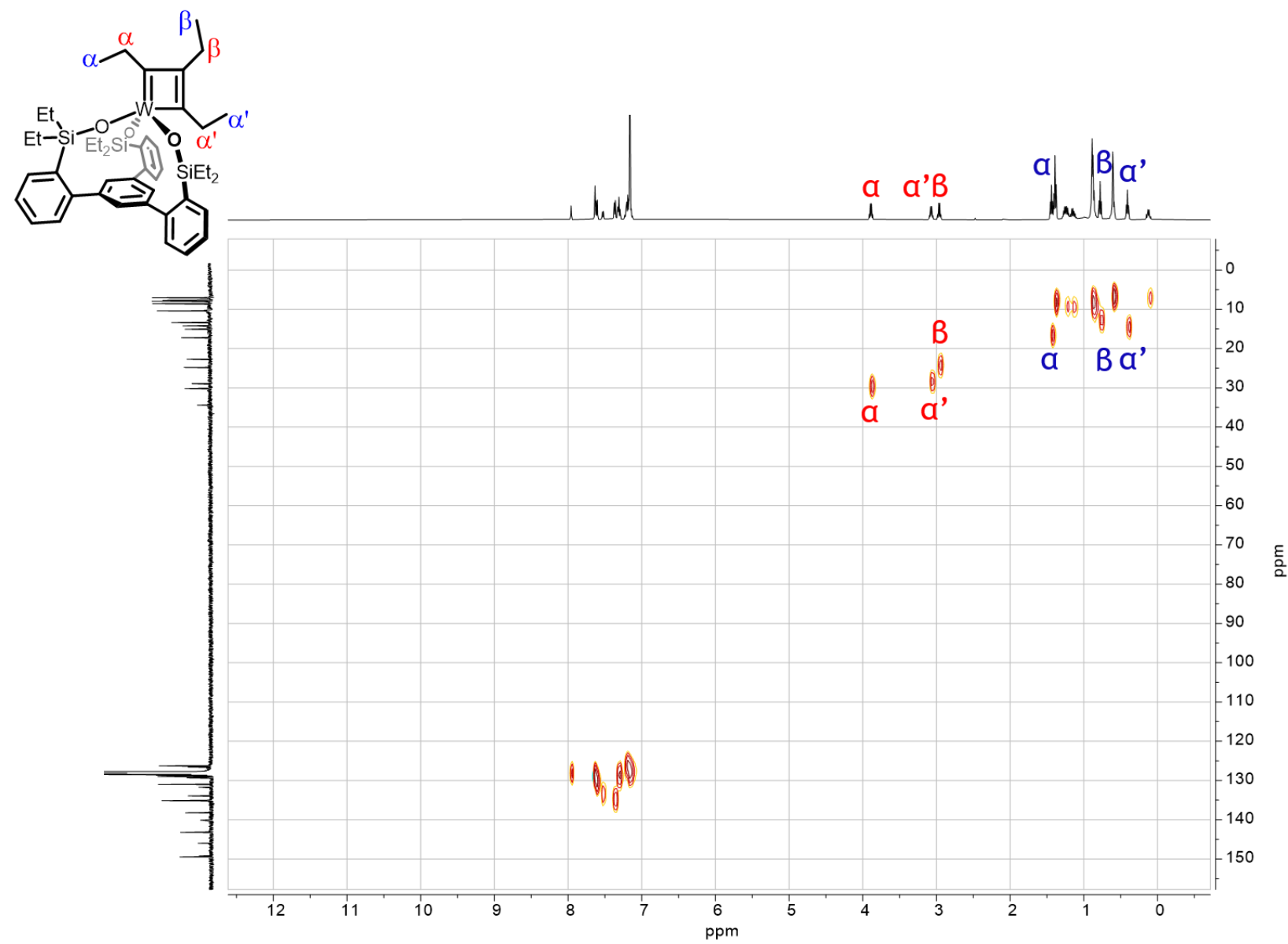

Figure S33. One-bond coupling ${ }^{1} \mathrm{H}^{-13} \mathrm{C}$ gHSQC NMR (500 MHz, $\mathrm{C}_{6} \mathrm{D}_{6}, 25{ }^{\circ} \mathrm{C}$ ) spectrum of MCBD5. 


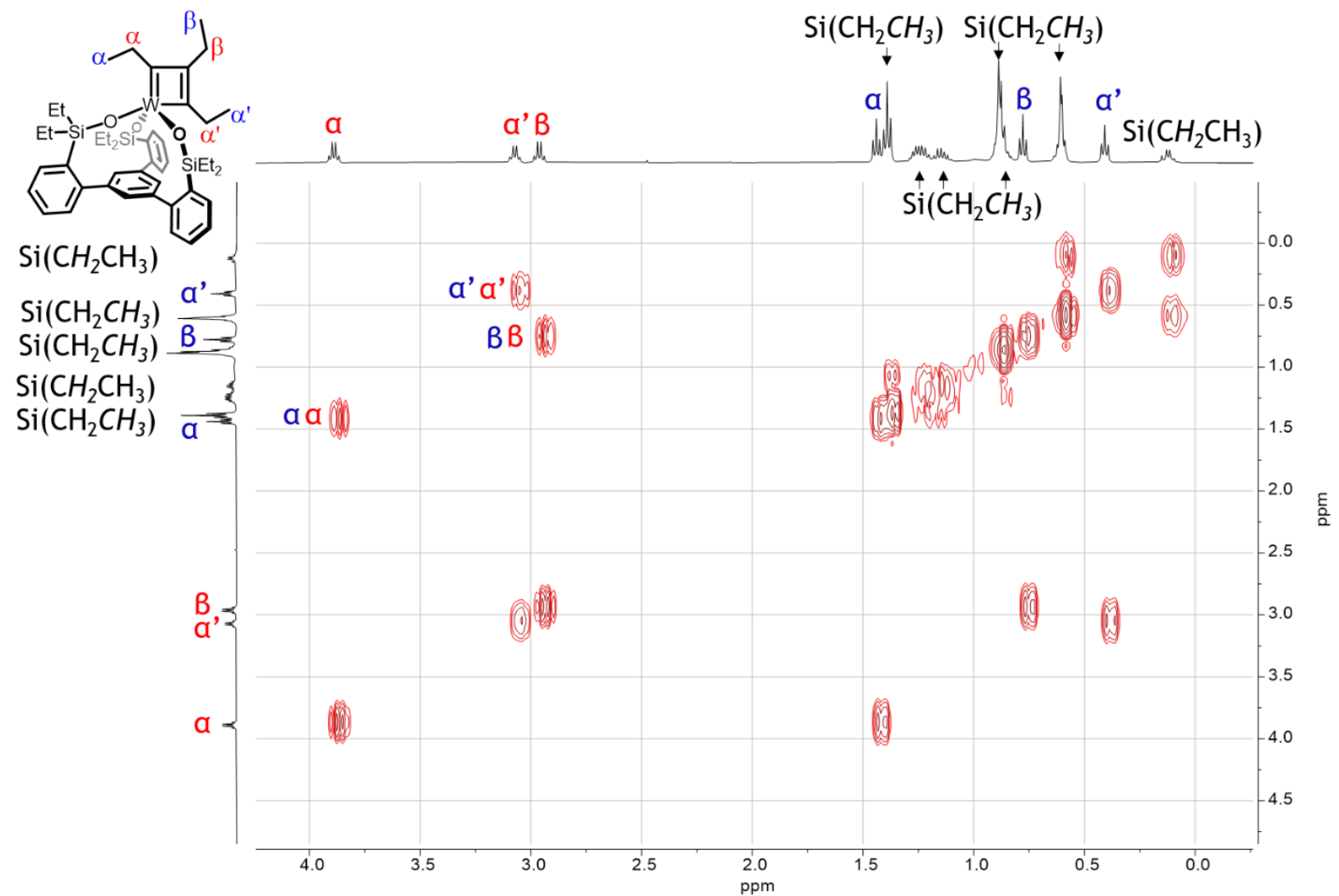

Figure S34. Long-range coupling ${ }^{1} \mathrm{H}_{-}{ }^{13} \mathrm{C}$ gHMBC NMR $\left(500 \mathrm{MHz}, \mathrm{C}_{6} \mathrm{D}_{6}, 25{ }^{\circ} \mathrm{C}\right)$ spectrum of MCBD5. 


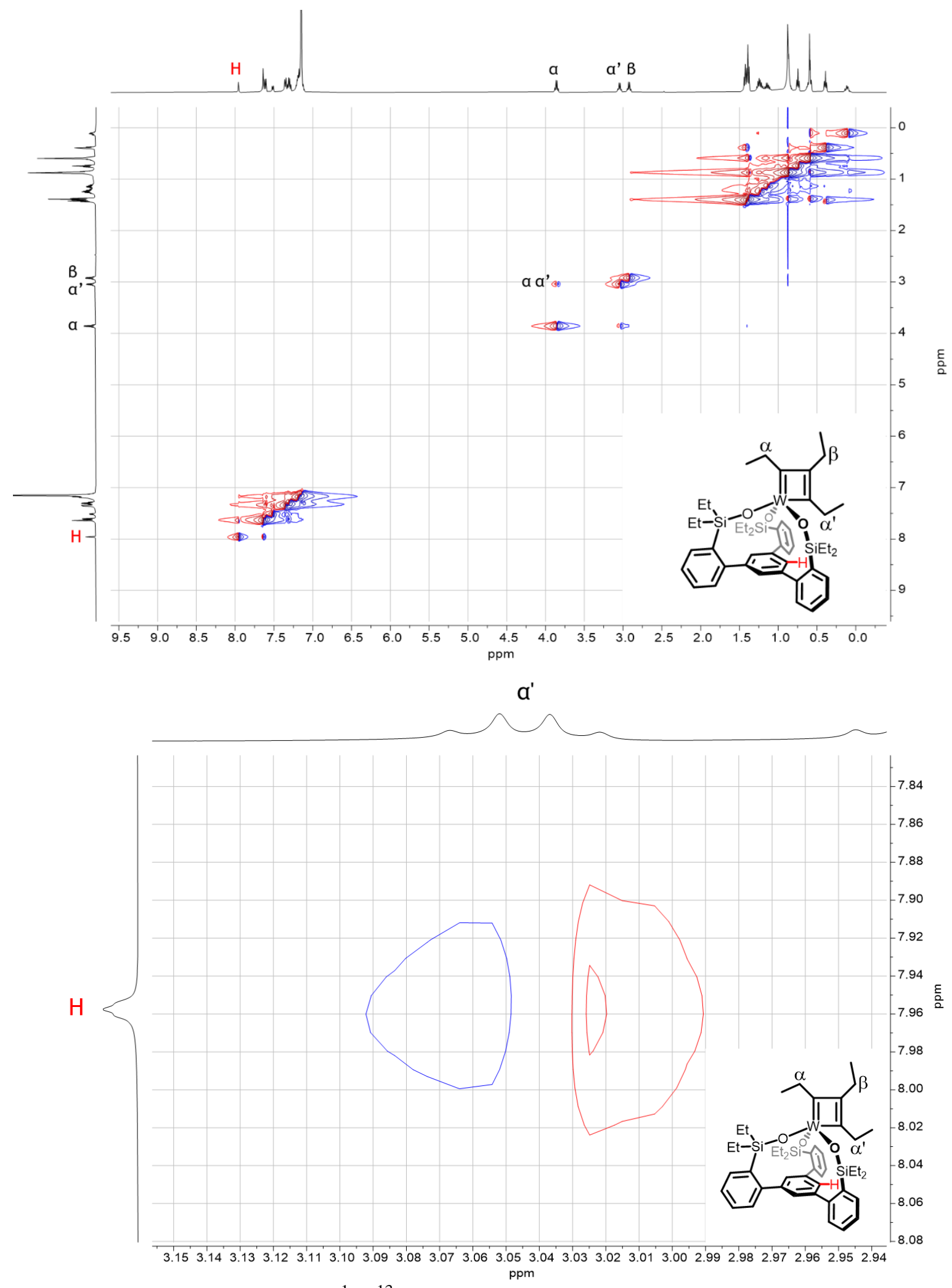

Figure S35. Long-range coupling ${ }^{1} \mathrm{H}-{ }^{13} \mathrm{C}$ gNOESY NMR $\left(500 \mathrm{MHz}, \mathrm{C}_{6} \mathrm{D}_{6}, 25^{\circ} \mathrm{C}\right)$ spectrum of MCBD5 (Top). Expanded portion showing correlation of ${ }^{1} \mathrm{H} \alpha^{\prime} \mathrm{CH}_{2}$ with the basal arene proton. (Bottom). 

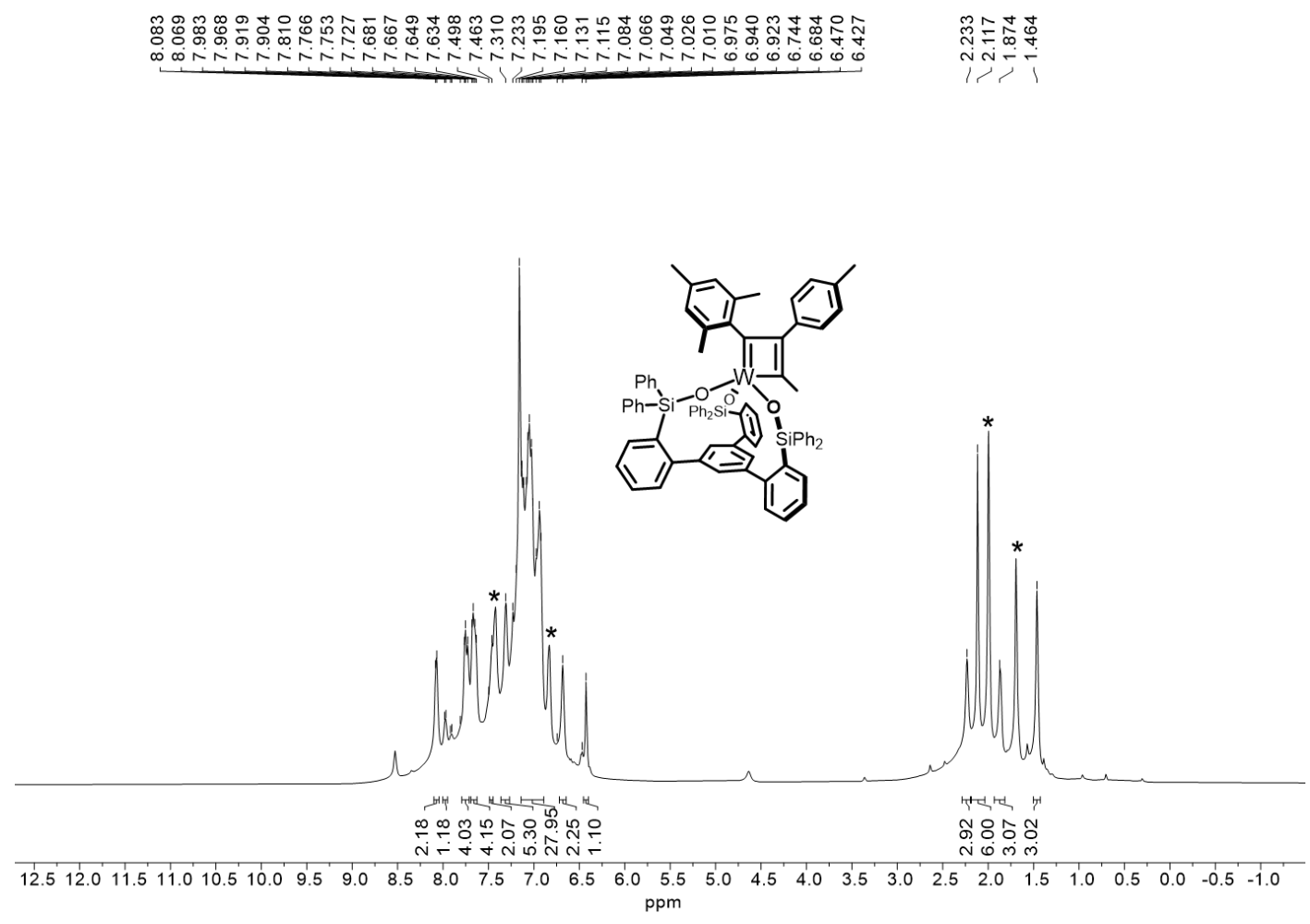

Figure S36. ${ }^{1} \mathrm{H}$ NMR $\left(500 \mathrm{MHz}, \mathrm{C}_{6} \mathrm{D}_{6}, 25^{\circ} \mathrm{C}\right)$ spectrum of MCBD6. * Denotes excess $p$ tolylpropyne. 


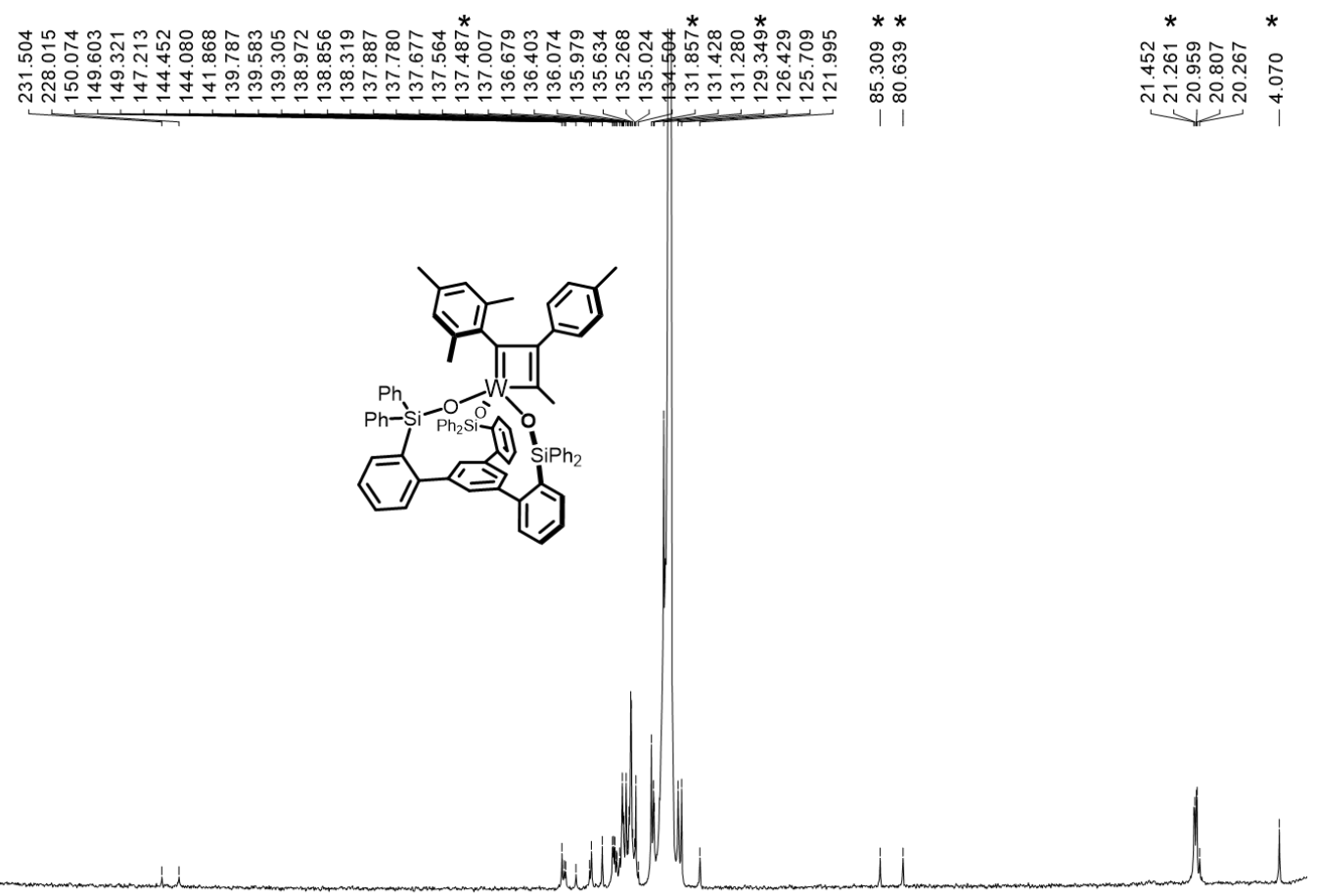

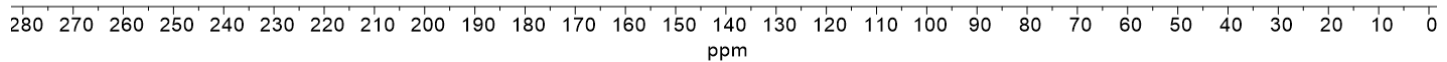

Figure S37. ${ }^{13} \mathrm{C}$ NMR $\left(125 \mathrm{MHz}, \mathrm{C}_{6} \mathrm{D}_{6}, 25{ }^{\circ} \mathrm{C}\right)$ spectrum of MCBD6. $*$ Denotes excess $p$ tolylpropyne.

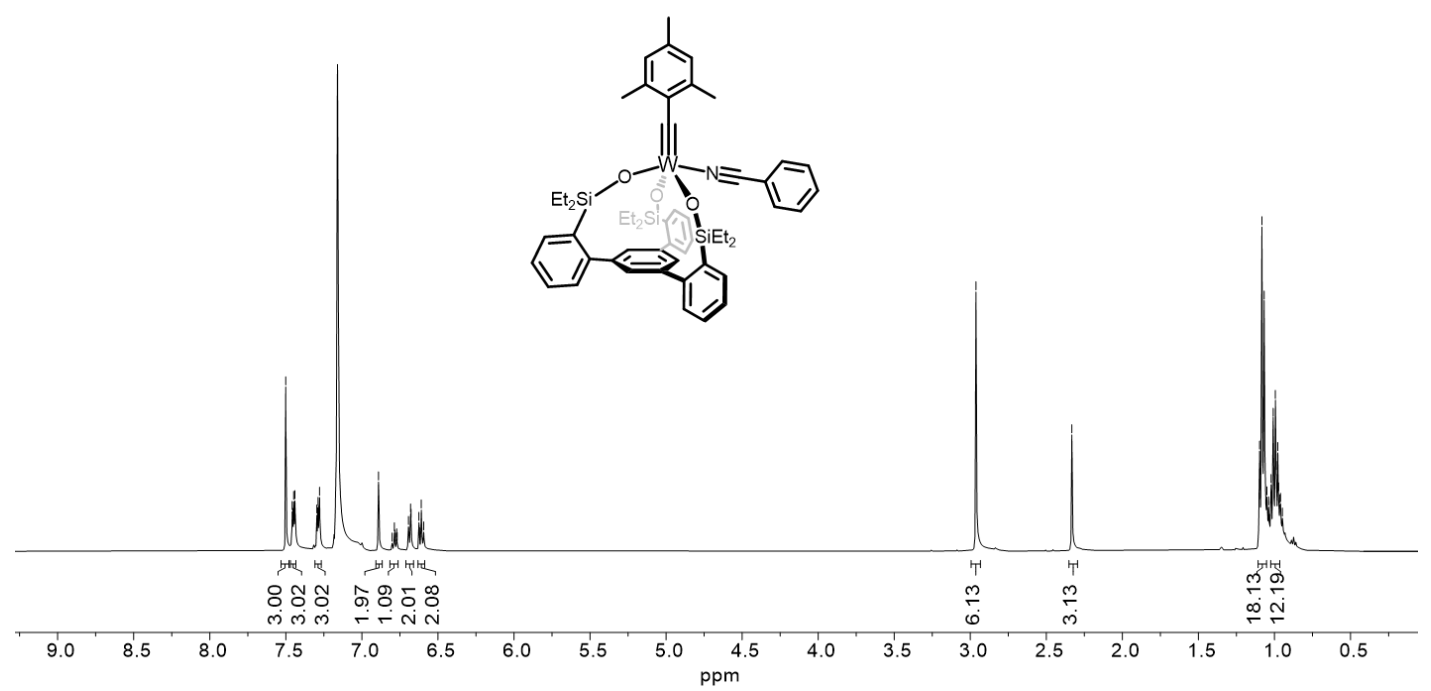

Figure S38. ${ }^{1} \mathrm{H}$ NMR $\left(500 \mathrm{MHz}, \mathrm{C}_{6} \mathrm{D}_{6}, 25{ }^{\circ} \mathrm{C}\right)$ spectrum of Cat5•PhCN. 


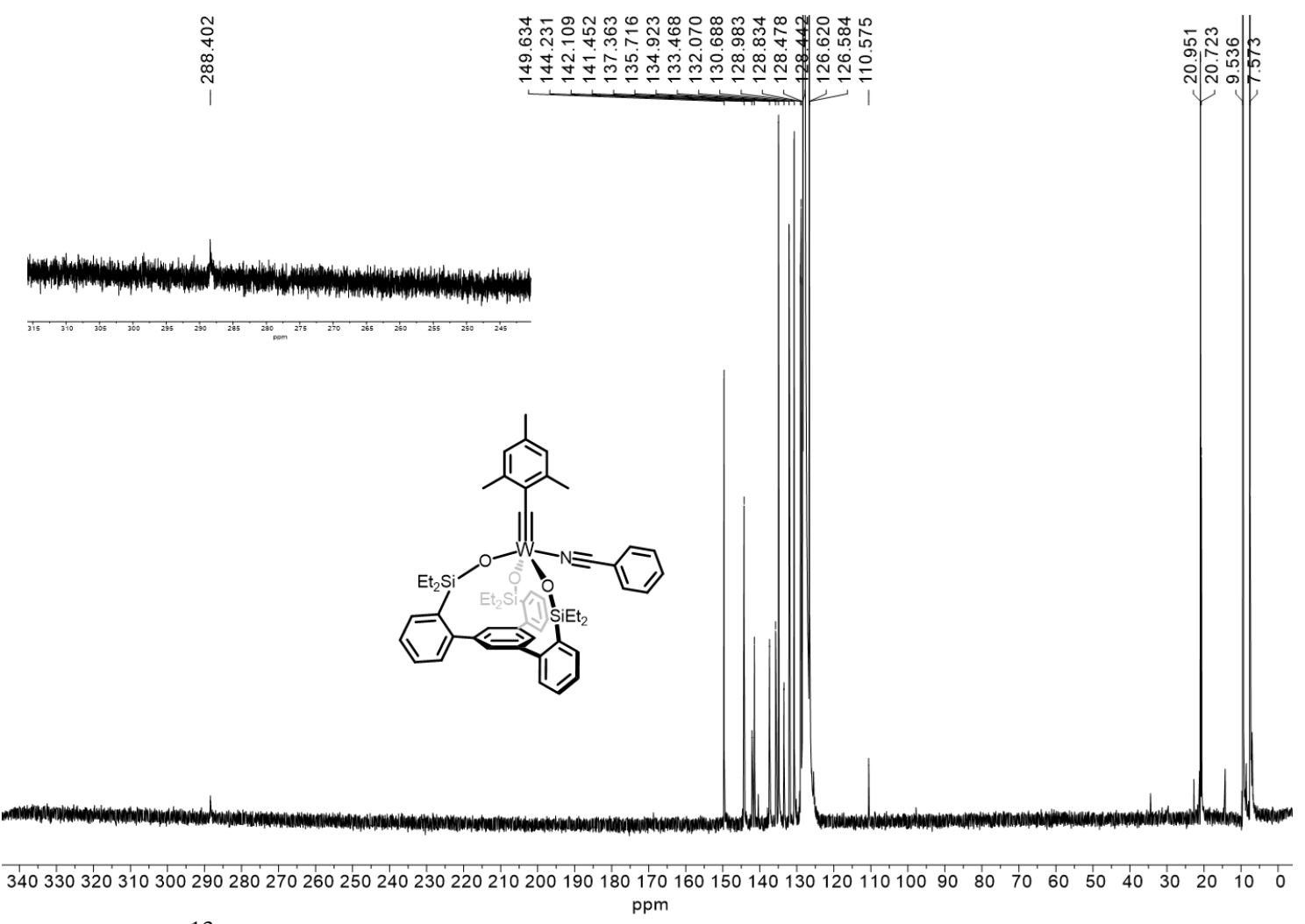

Figure S39. ${ }^{13} \mathrm{C}$ NMR $\left(125 \mathrm{MHz}, \mathrm{C}_{6} \mathrm{D}_{6}, 25{ }^{\circ} \mathrm{C}\right)$ spectrum of Cat5•PhCN. 


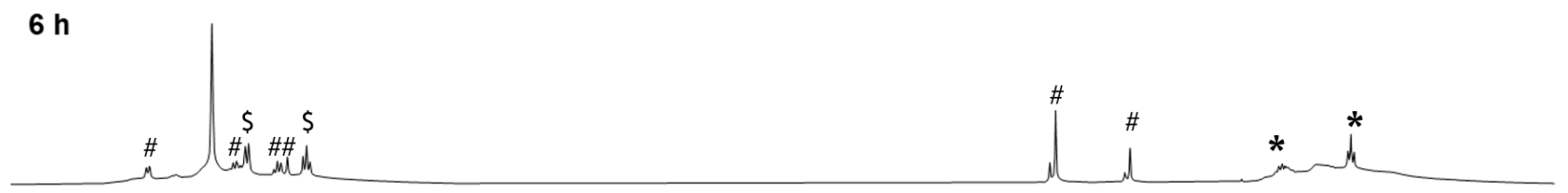

$3 \mathbf{h}$

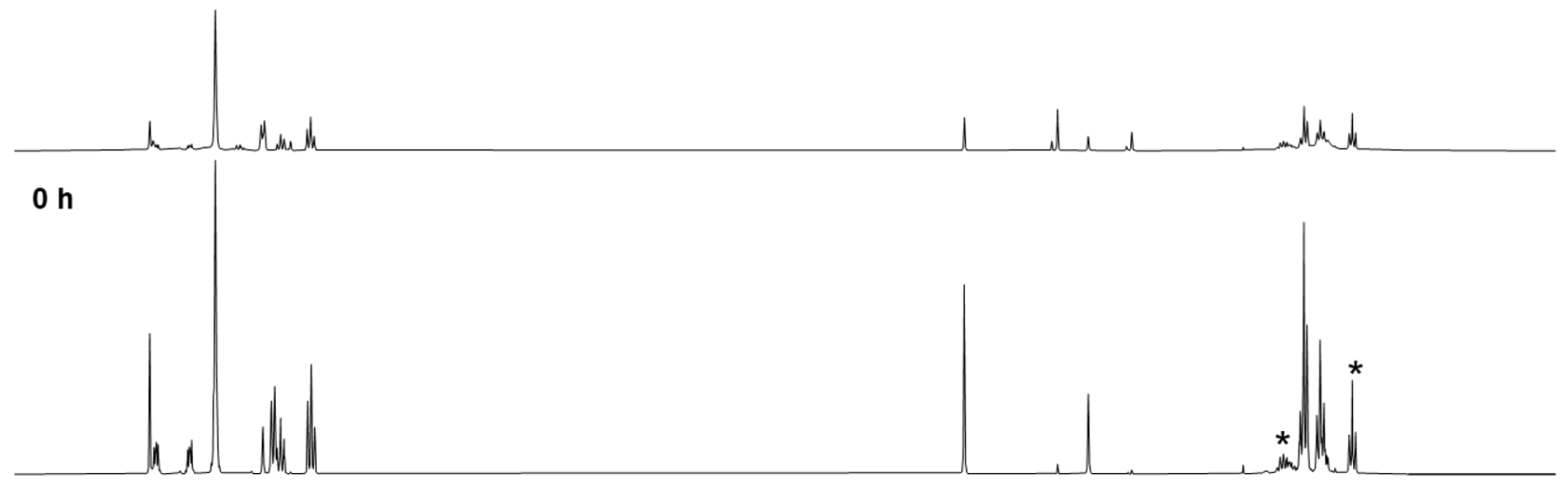

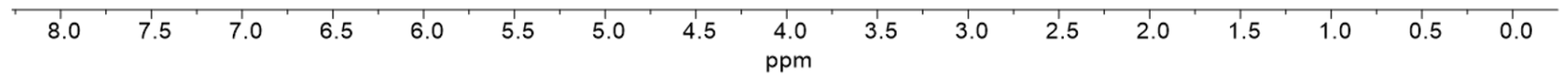

Figure S40. ${ }^{1} \mathrm{H}$ NMR $\left(400 \mathrm{MHz}, \mathrm{C}_{6} \mathrm{D}_{6}, 25{ }^{\circ} \mathrm{C}\right)$ decomposition of Cat5•PhCN to Nitride1 and mesityl-phenylacetylene over the course of $6 \mathrm{~h}$. * Denotes pentane impurity, \# denotes mesitylphenylacetylene and $\$$ denotes residual benzonitrile.

\section{$\underline{\text { Rate of Alkyne Metathesis using Mo(VI) and W(VI)-based SiP Catalysts }}$}

Under an inert atmosphere, a $0.2 \mathrm{mM} / 0.002 \mathrm{mM}$ solution of substrate/catalyst was prepared in 0.6 $\mathrm{mL} \mathrm{C}_{6} \mathrm{D}_{6}$. The percentage of substrate conversion was monitored by integration of the $\mathrm{OCH}_{3}$ resonance periodically at set time points. The MestraNova fitting function was used to assist in the integration. 


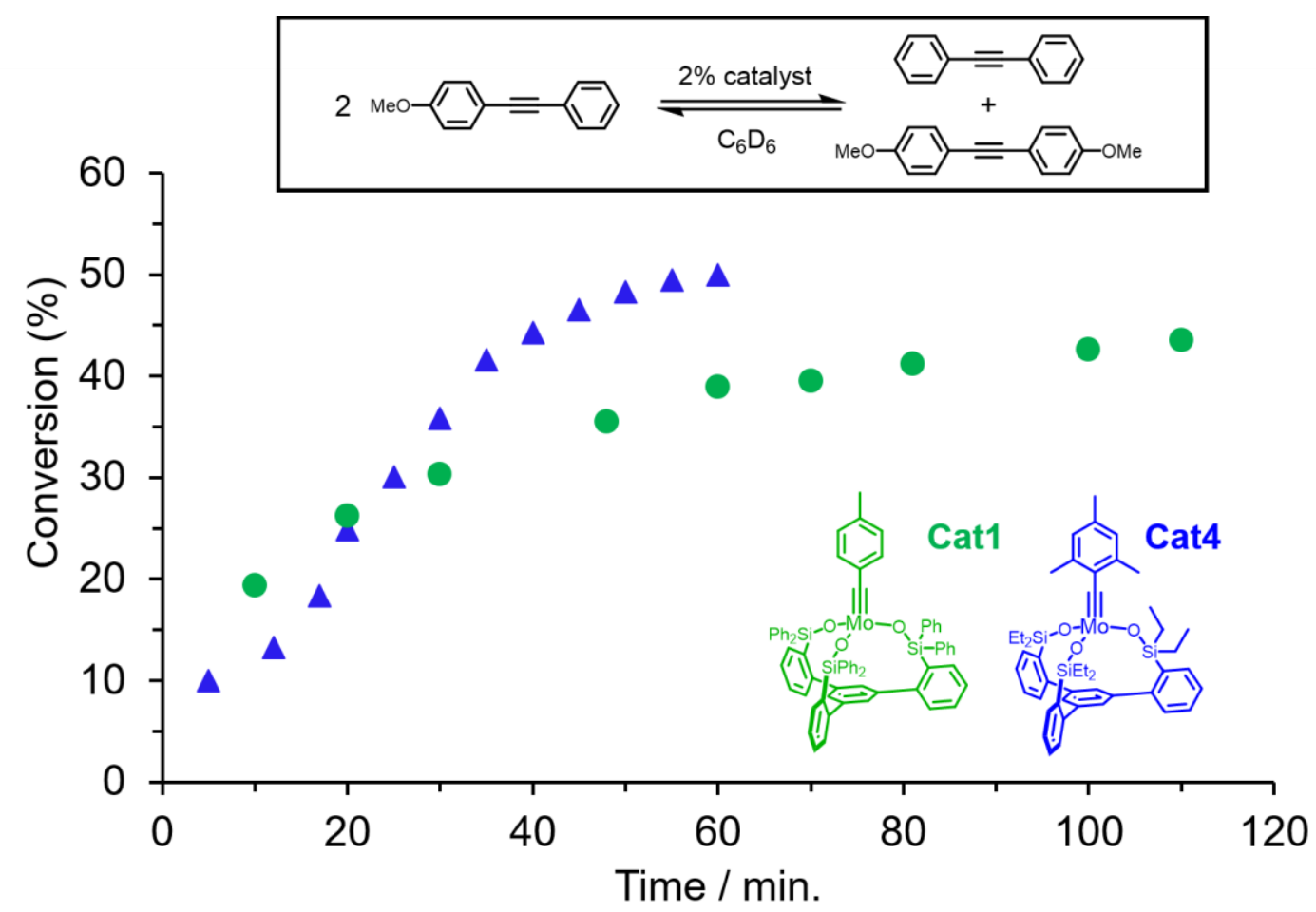

Figure S41. Dynamic scrambling of 1-methoxy-4-(phenylethynl)benzene $\left(0.1 \mathrm{mM}\right.$ in $\left.\mathrm{C}_{6} \mathrm{D}_{6}\right)$ catalyzed by $2 \mathrm{~mol} \%$ of Cat1 and Cat4 at rt monitored by ${ }^{1} \mathrm{H}$ NMR.
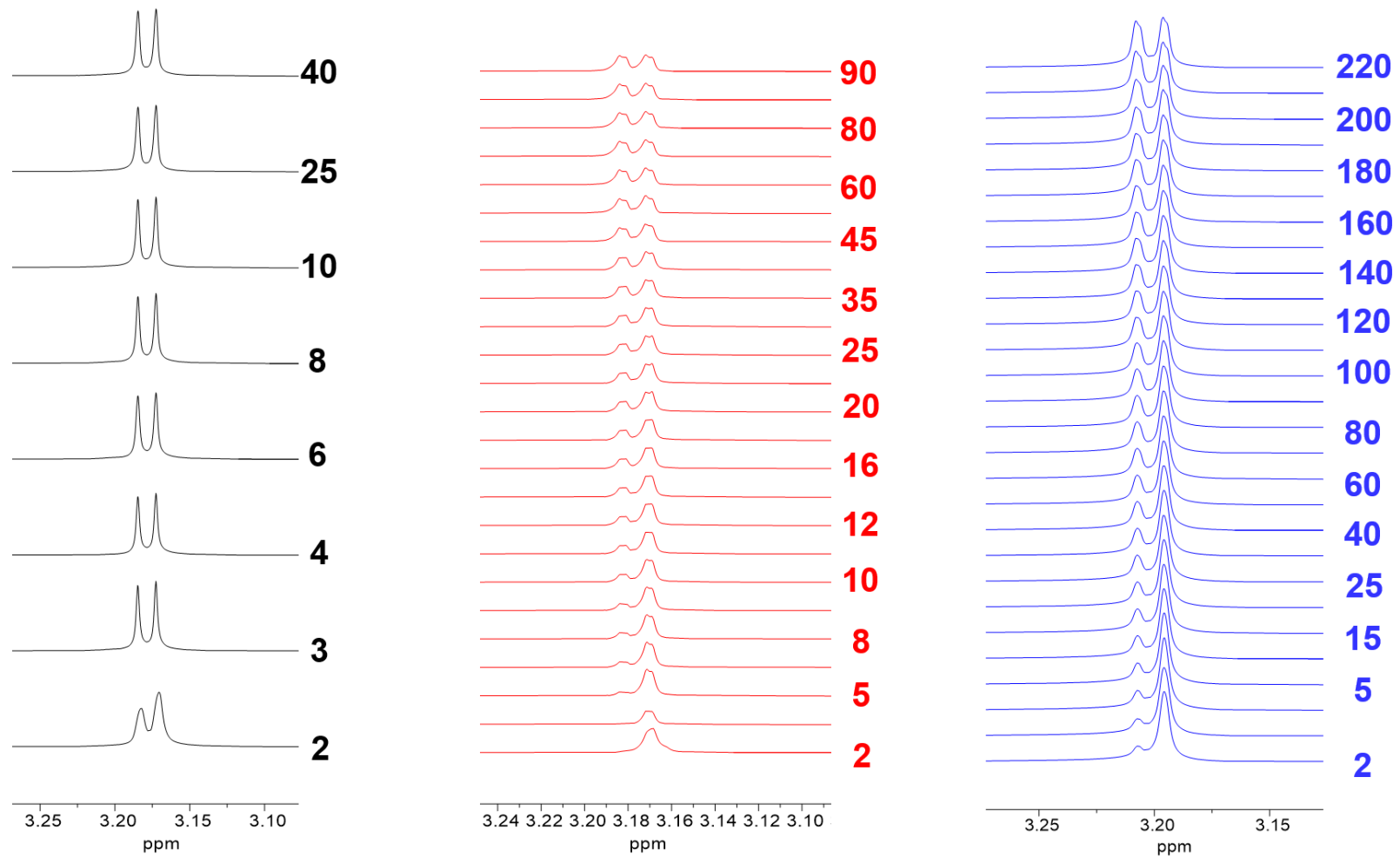

Figure S42. Stacked ${ }^{1} \mathrm{H}$ NMR plots for kinetics experiments using Cat5 (Left, Black), Cat3 (Center, Red) and Cat 4 (Right, Blue). Integrations were obtained by "Generalized Lorentzian" fitting in mnova NMR software. 


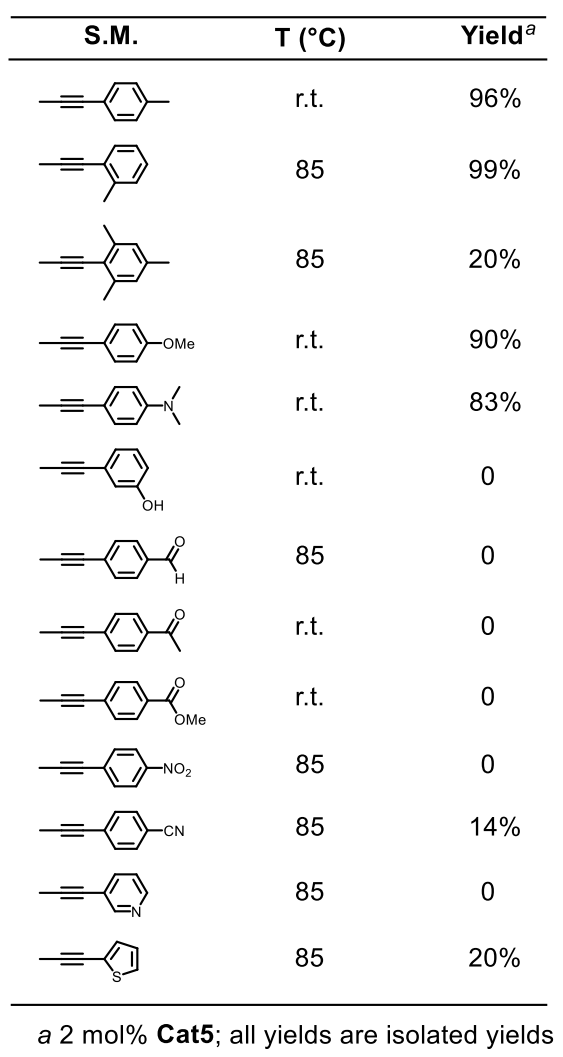

Figure S43. Substrate Scope using Cat5.

\section{Crystallographic Information}

Crystallographic data are summarized in Tables S1-S4. Suitable crystals for X-ray analysis of SiP ${ }^{\mathrm{E}}$, Pre2, Cat3-5, MTd2, MCBD2-6, Cat5•PhCN and Nitride1 were placed MiTeGen pins, coated in oil. The X-ray intensity data collection was carried out on a Bruker APEXII DUO CCD area detector using graphite-monochromated Mo-K $\alpha$ radiation $(\lambda=0.71073 \AA)$ or $\mathrm{Cu}-\mathrm{K} \alpha$ radiation $(\lambda=1.54184 \AA)$ at $90.0(5) \mathrm{K}$. Frames were integrated using SAINT, ${ }^{6}$ producing a listing of non-averaged $\mathrm{F}^{2}$ and $\sigma\left(\mathrm{F}^{2}\right)$ values. The intensity data were corrected for Lorentz and polarization effects and for absorption using SADABS. ${ }^{7}$ The initial structure was determined by intrinsic phasing using SHELXT. ${ }^{8}$ The further structure determination was performed by difference Fourier methods and refined by full-matrix least squares using SHELXL ${ }^{9}$ or olex2.refine for Nitride1. All reflections were used during refinements. Non-hydrogen atoms were refined anisotropically and hydrogen atoms were visible in difference maps, but placed in idealized positions and treated with riding models. Disordered phenyl groups successfully modeled. Disordered solvent was removed using the SQUEEZE procedure for Cat3, MCBD4 and MTd2. 


\section{Table S1. Crystallographic Data for SiPEt, Cat3-5}

\begin{tabular}{|c|c|c|c|c|}
\hline & $\mathbf{S i P}^{\mathrm{Et}}$ & Cat3 & Cat4 & Cat5 \\
\hline Molecular Formula & $\mathrm{C}_{72} \mathrm{H}_{96} \mathrm{O}_{6} \mathrm{Si} 6$ & $\mathrm{C}_{70} \mathrm{H}_{56} \mathrm{O}_{3} \mathrm{Si}_{3} \mathrm{~W}$ & $\mathrm{C}_{46} \mathrm{H}_{56} \mathrm{MoO}_{3} \mathrm{Si}_{3}$ & $\begin{array}{c}\mathrm{C}_{46} \mathrm{H}_{56} \mathrm{O}_{3} \mathrm{Si}_{3} \mathrm{~W} \cdot \\
\left(\mathrm{C}_{5} \mathrm{H}_{12}\right)_{0.5}\end{array}$ \\
\hline Fw & 1226.02 & 1489.67 & 837.11 & 961.10 \\
\hline $\operatorname{Temp}(\mathrm{K})$ & $90.0(5)$ & $90.0(5)$ & $90.0(5)$ & $90.0(5)$ \\
\hline Crystal System & Triclinic & Triclinic & Monoclinic & Monoclinic \\
\hline Space Group & P-1 & P-1 & $\mathrm{P} 21 / \mathrm{n}$ & $\mathrm{P} 21 / \mathrm{n}$ \\
\hline \multicolumn{5}{|l|}{ Cell Constants } \\
\hline$a(\AA)$ & $8.9495(12)$ & $13.3644(11)$ & $12.2321(4)$ & $21.4428(8)$ \\
\hline$b(\AA)$ & $11.8064(15)$ & $13.7952(11)$ & $23.6045(9)$ & $16.0018(6)$ \\
\hline$c(\AA)$ & $35.142(4)$ & $21.6377(17)$ & $15.0674(5)$ & $27.6488(10)$ \\
\hline$\alpha(\operatorname{deg})$ & $93.460(7)$ & $105.889(3)$ & 90 & 90 \\
\hline$\beta(\operatorname{deg})$ & $90.523(8)$ & $95.015(3)$ & $96.909(2)$ & $104.890(2)$ \\
\hline$\gamma(\operatorname{deg})$ & $111.376(7)$ & $104.262(3)$ & 90 & 90 \\
\hline$Z$ & 2 & 2 & 4 & 8 \\
\hline$V\left(\AA^{3}\right)$ & $3449.5(8)$ & $3666.9(5)$ & $4318.9(3)$ & $9168.4(6)$ \\
\hline Abs Coeff, $\mu_{\text {calc }}\left(\mathrm{mm}^{-1}\right)$ & 0.171 & 1.676 & 0.425 & 2.637 \\
\hline$\delta_{\text {calc }}\left(\mathrm{g} / \mathrm{cm}^{3}\right)$ & 1.180 & 1.349 & 1.287 & 1.393 \\
\hline $\mathrm{F}(000)\left(\mathrm{e}^{-} / \AA^{3}\right)$ & 1320 & 1532 & 1760 & 3944 \\
\hline Crystal Dimensions (mm) & $\begin{array}{c}1.330 \times 0.830 \\
\times 0.550\end{array}$ & $0.14 \times 0.13 \times 0.09$ & $0.17 \times 0.15 \times 0.08$ & $0.17 \times 0.14 \times 0.09$ \\
\hline Radiation & $\begin{array}{c}\text { Mo K } \alpha \\
(\lambda=0.71073 \\
\AA)\end{array}$ & $\begin{array}{c}\text { Mo K } \alpha \\
(\lambda=0.71073 \AA)\end{array}$ & $\begin{array}{c}\text { Mo K } \alpha \\
(\lambda=0.71073 \AA)\end{array}$ & $\begin{array}{c}\text { Mo K } \alpha \\
(\lambda=0.71073 \AA)\end{array}$ \\
\hline$h, k, l$ Ranges Collected & $\begin{array}{c}-11 \leq \mathrm{h} \leq 11 \\
-14 \leq \mathrm{k} \leq 14 \\
-43 \leq 1 \leq 43\end{array}$ & $\begin{array}{c}-19 \leq \mathrm{h} \leq 20,-22 \leq \mathrm{k} \leq 21,- \\
33 \leq 1 \leq 30\end{array}$ & $\begin{array}{c}-20 \leq \mathrm{h} \leq 20,-39 \leq \mathrm{k} \\
\leq 39,-24 \leq 1 \leq 25\end{array}$ & $\begin{array}{c}-30 \leq \mathrm{h} \leq 30,-22 \leq \mathrm{k} \leq \\
22,-39 \leq 1 \leq 39\end{array}$ \\
\hline$\theta$ Range (deg) & $1.743-26.352$ & $1.594-34.723$ & $1.612-36.386$ & $1.390-30.587$ \\
\hline $\begin{array}{l}\text { No. of Reflections } \\
\text { Collected }\end{array}$ & 19894 & 23848 & 144408 & 28138 \\
\hline $\begin{array}{l}\text { No. of Unique } \\
\text { Reflections }\end{array}$ & 12605 & 12699 & 15695 & 19345 \\
\hline No. of Parameters & 772 & 697 & 487 & 1092 \\
\hline Data/Parameter Ratio & 16.33 & 18.22 & 32.23 & 17.72 \\
\hline Refinement Method & $\begin{array}{l}\text { Full-matrix } \\
\text { least-squares } \\
\text { of } F^{2}\end{array}$ & $\begin{array}{l}\text { Full-matrix } \\
\text { least-squares of } F^{2}\end{array}$ & $\begin{array}{c}\text { Full-matrix } \\
\text { least-squares of } F^{2}\end{array}$ & $\begin{array}{c}\text { Full-matrix } \\
\text { least-squares of } F^{2}\end{array}$ \\
\hline $\mathrm{R}(F)^{\mathrm{a}}$ & 0.0580 & 0.0590 & 0.0357 & 0.0475 \\
\hline $\mathrm{R}_{w}\left(F^{2}\right)^{\mathrm{b}}$ & 0.1648 & 0.1159 & 0.0745 & 0.1074 \\
\hline $\mathrm{GOF}_{\mathrm{w}}{ }^{\mathrm{c}}$ & 0.983 & 0.867 & 1.012 & 1.062 \\
\hline $\begin{array}{l}\text { Largest Diff Peak and } \\
\text { Hole }\left(\mathrm{e}^{-} / \AA^{3}\right)\end{array}$ & $\begin{array}{l}0.809 \text { and - } \\
0.418\end{array}$ & 0.621 and -0.584 & 0.854 and -0.913 & 3.104 and -1.732 \\
\hline
\end{tabular}


Table S2. Crystallographic Data for Pre2, MTd2, MCBD2,3

\begin{tabular}{|c|c|c|c|c|}
\hline & Pre2 & $\mathbf{M T}_{\mathbf{d}} 2$ & MCBD2 & MCBD3 \\
\hline Molecular Formula & $\mathrm{C}_{22} \mathrm{H}_{38} \mathrm{O}_{3} \mathrm{~W}$ & $\mathrm{C}_{75} \mathrm{H}_{72} \mathrm{MoO}_{3} \mathrm{Si}_{3}$ & $\mathrm{C}_{81} \mathrm{H}_{78} \mathrm{O}_{3} \mathrm{Si}_{3} \mathrm{~W}$ & $\mathrm{C}_{52} \mathrm{H}_{66} \mathrm{O}_{3} \mathrm{Si}_{3} \mathrm{~W}$ \\
\hline Fw & 534.37 & 1201.53 & 1367.55 & 1007.16 \\
\hline $\operatorname{Temp}(\mathrm{K})$ & $90.0(5)$ & $90.0(5)$ & $90.0(5)$ & $90.0(5)$ \\
\hline Crystal System & Triclinic & Trigonal & Triclinic & Monoclinic \\
\hline Space Group & P-1 & P-3 & P-1 & $\mathrm{P} 21$ \\
\hline Cell Constants & & & & \\
\hline$a(\AA)$ & $9.6691(15)$ & $21.8844(12)$ & $12.6748(19)$ & $11.0934(3)$ \\
\hline$b(\AA ⿻)$ & $11.4794(18)$ & $21.8844(12)$ & $14.118(2)$ & $38.3571(10)$ \\
\hline$c(\AA)$ & 12.0496(19) & $10.3174(4)$ & $19.313(3)$ & $11.4648(3)$ \\
\hline$\alpha(\operatorname{deg})$ & $106.175(2)$ & 90 & $90.047(8)$ & 90 \\
\hline$\beta(\operatorname{deg})$ & $102.620(2)$ & 90 & $99.423(8)$ & $100.574(2)$ \\
\hline$\gamma(\operatorname{deg})$ & $102.920(2)$ & 120 & $101.919(8)$ & 90 \\
\hline$Z$ & 2 & 2 & 2 & 4 \\
\hline$V\left(\AA^{3}\right)$ & $1194.3(3)$ & $4279.3(5)$ & $3333.6(8)$ & $4795.6(2)$ \\
\hline $\begin{array}{l}\text { Abs Coeff, } \mu_{\text {calc }}\left(\mathrm{mm}^{-}\right. \\
\left.{ }^{1}\right)\end{array}$ & 4.852 & 4.864 & 1.836 & 2.525 \\
\hline$\delta_{\text {calc }}\left(\mathrm{g} / \mathrm{cm}^{3}\right)$ & 1.486 & 1.262 & 1.362 & 1.395 \\
\hline $\mathrm{F}(000)\left(\mathrm{e}^{-} / \AA^{3}\right)$ & 536 & 1680 & 1408 & 2072 \\
\hline $\begin{array}{l}\text { Crystal Dimensions } \\
(\mathrm{mm})\end{array}$ & $0.279 \times 0.243 \times 0.067$ & $0.160 \times 0.060 \times 0.050$ & $0.397 \times 0.267 \times 0.156$ & $0.19 \times 0.18 \times 0.10$ \\
\hline Radiation & $\begin{array}{c}\text { Mo K } \alpha \\
(\lambda=0.71073 \AA)\end{array}$ & $\begin{array}{c}\mathrm{Cu} \mathrm{K \alpha} \\
(\lambda=1.54184 \AA)\end{array}$ & $\begin{array}{c}\text { Mo K } \alpha \\
(\lambda=0.71073 \AA)\end{array}$ & $\begin{array}{c}\text { Mo K } \alpha \\
(\lambda=0.71073 \AA)\end{array}$ \\
\hline$h, k, l$ Ranges Collected & $\begin{array}{c}-13 \leq \mathrm{h} \leq 13,-15 \leq \mathrm{k} \\
\leq 15,-16 \leq 1 \leq 16\end{array}$ & $\begin{array}{c}-24 \leq \mathrm{h} \leq 24,-24 \leq \mathrm{k} \leq \\
24,-11 \leq 1 \leq 11\end{array}$ & $\begin{array}{c}-15 \leq \mathrm{h} \leq 15,-17 \leq \mathrm{k} \leq \\
17,-23 \leq 1 \leq 23\end{array}$ & $\begin{array}{c}-16 \leq \mathrm{h} \leq 16,-56 \leq \mathrm{k} \\
\leq 56,-14 \leq 1 \leq 16\end{array}$ \\
\hline$\theta$ Range (deg) & $1.845-28.929$ & $2.331-61.236$ & $1.666-25.682$ & $1.062-31.5 \overline{4} 4$ \\
\hline $\begin{array}{l}\text { No. of Reflections } \\
\text { Collected }\end{array}$ & 6259 & 4389 & 11380 & 26810 \\
\hline $\begin{array}{l}\text { No. of Unique } \\
\text { Reflections }\end{array}$ & 5982 & 4080 & 6717 & 25100 \\
\hline No. of Parameters & 247 & 249 & 800 & 1071 \\
\hline Data/Parameter Ratio & 24.22 & 17.63 & 8.40 & 23.44 \\
\hline Refinement Method & $\begin{array}{c}\text { Full-matrix } \\
\text { least-squares of } F^{2}\end{array}$ & $\begin{array}{c}\text { Full-matrix } \\
\text { least-squares of } F^{2}\end{array}$ & $\begin{array}{c}\text { Full-matrix } \\
\text { least-squares of } F^{2}\end{array}$ & $\begin{array}{c}\text { Full-matrix } \\
\text { least-squares of } F^{2}\end{array}$ \\
\hline $\mathrm{R}(F)^{\mathrm{a}}$ & 0.0286 & 0.0948 & 0.0509 & 0.0669 \\
\hline $\mathrm{R}_{w}\left(F^{2}\right)^{\mathrm{b}}$ & 0.0752 & 0.22 .93 & 0.0962 & 0.1512 \\
\hline $\mathrm{GOF}_{\mathrm{w}} \mathrm{c}^{\mathrm{c}}$ & 1.182 & 1.151 & 0.882 & 1.187 \\
\hline $\begin{array}{l}\text { Largest Diff Peak and } \\
\text { Hole }\left(\mathrm{e}^{-} / \AA^{3}\right)\end{array}$ & 4.292 and -2.795 & 1.487 and -0.880 & 1.798 and -1.704 & 5.494 and -6.780 \\
\hline
\end{tabular}


Table S3. Crystallographic Data for MCBD4-6 and Cat5•PhCN

\begin{tabular}{|c|c|c|c|c|}
\hline & MCBD4 & MCBD5 & MCBD6 & Cat5•PhCN \\
\hline Molecular Formula & $\begin{array}{c}\mathrm{C}_{69} \mathrm{H}_{60} \mathrm{O}_{3} \mathrm{Si}_{3} \mathrm{~W} \cdot \\
\quad\left(\mathrm{CH}_{2} \mathrm{Cl}_{2}\right)\end{array}$ & $\mathrm{C}_{45} \mathrm{H}_{60} \mathrm{O}_{3} \mathrm{Si}_{3} \mathrm{~W}$ & $\mathrm{C}_{80} \mathrm{H}_{66} \mathrm{O}_{3} \mathrm{Si}_{3} \mathrm{~W}$ & $\mathrm{C}_{53} \mathrm{H}_{61} \mathrm{NO}_{3} \mathrm{Si}_{3} \mathrm{~W}$ \\
\hline Fw & 1529.83 & 917.05 & 1343.44 & 1028.14 \\
\hline $\operatorname{Temp}(\mathrm{K})$ & $90.0(5)$ & $90.0(5)$ & $90.0(5)$ & $90.0(5)$ \\
\hline Crystal System & Triclinic & Monoclinic & Monoclinic & Triclinic \\
\hline Space Group & $\mathrm{P}-1$ & $\mathrm{P} 2{ }_{1} / \mathrm{c}$ & $\mathrm{P} 2{ }_{1} / \mathrm{n}$ & $\mathrm{P}-1$ \\
\hline \multicolumn{5}{|l|}{ Cell Constants } \\
\hline$a(\AA)$ & $12.9871(11)$ & $9.7250(6)$ & $13.5425(4)$ & $10.7732(8)$ \\
\hline$b(\AA)$ & $14.2927(12)$ & $26.0899(16)$ & $19.4843(6)$ & 11.2917(9) \\
\hline$c(\AA)$ & $18.5412(15)$ & $17.2191(12)$ & $24.2912(7)$ & $20.8166(16)$ \\
\hline$\alpha(\operatorname{deg})$ & $91.487(4)$ & 90 & 90 & 76.731(3) \\
\hline$\beta(\operatorname{deg})$ & $95.290(4)$ & $106.222(2)$ & 104.3910(10) & $76.456(3)$ \\
\hline$\gamma(\operatorname{deg})$ & $95.748(4)$ & 90 & 90 & $82.248(3)$ \\
\hline$Z$ & 2 & 4 & 4 & 2 \\
\hline$V\left(\AA^{3}\right)$ & $3407.6(5)$ & $4195.0(5)$ & $6208.5(3)$ & $2387.3(3)$ \\
\hline $\begin{array}{l}\text { Abs Coeff, } \mu_{\text {calc }}\left(\mathrm{mm}^{-}\right. \\
\left.{ }^{1}\right)\end{array}$ & 2.095 & 2.878 & 1.971 & 2.538 \\
\hline$\delta_{\text {calc }}\left(\mathrm{g} / \mathrm{cm}^{3}\right)$ & 1.491 & 1.452 & 1.437 & 1.430 \\
\hline $\mathrm{F}(000)\left(\mathrm{e}^{-} / \AA^{3}\right)$ & 1549 & 1880 & 2744 & 1052 \\
\hline $\begin{array}{l}\text { Crystal Dimensions } \\
(\mathrm{mm})\end{array}$ & $0.342 \times 0.174 \times 0.166$ & $0.124 \times 0.113 \times 0.051$ & $0.102 \times 0.070 \times 0.020$ & $0.180 \times 0.160 \times 0.070$ \\
\hline Radiation & $\begin{array}{c}\text { Mo K } \alpha \\
(\lambda=0.71073 \AA)\end{array}$ & $\begin{array}{c}\text { Mo K } \alpha \\
(\lambda=0.71073 \AA)\end{array}$ & $\begin{array}{c}\text { Mo K } \alpha \\
(\lambda=0.71073 \AA)\end{array}$ & $\begin{array}{c}\text { Mo K } \alpha \\
(\lambda=0.71073 \AA)\end{array}$ \\
\hline$h, k, l$ Ranges & $-25 \leq \mathrm{h} \leq 25,-28 \leq \mathrm{k} \leq$ & $-13 \leq \mathrm{h} \leq 14,-38 \leq \mathrm{k} \leq$ & $-15 \leq \mathrm{h} \leq 15,-20 \leq \mathrm{k} \leq$ & $-20 \leq \mathrm{h} \leq 20,-21 \leq \mathrm{k} \leq$ \\
\hline Collected & $28,-36 \leq 1 \leq 36$ & $37,-24 \leq 1 \leq 25$ & $22,-20 \leq 1 \leq 28$ & $21,-39 \leq 1 \leq 39$ \\
\hline$\theta$ Range (deg) & $1.583-44.391$ & $1.458-31.587$ & $1.357-24.778$ & $1.860-42.497$ \\
\hline $\begin{array}{l}\text { No. of Reflections } \\
\text { Collected }\end{array}$ & 54100 & 13285 & 10635 & 33277 \\
\hline $\begin{array}{l}\text { No. of Unique } \\
\text { Reflections }\end{array}$ & 38346 & 10695 & 7349 & 29979 \\
\hline No. of Parameters & 715 & 478 & 819 & 559 \\
\hline Data/Parameter Ratio & 53.63 & 22.37 & 8.97 & 59.5 \\
\hline Refinement Method & $\begin{array}{c}\text { Full-matrix } \\
\text { least-squares of } F^{2}\end{array}$ & $\begin{array}{c}\text { Full-matrix } \\
\text { least-squares of } F^{2}\end{array}$ & $\begin{array}{c}\text { Full-matrix } \\
\text { least-squares of } F^{2}\end{array}$ & $\begin{array}{c}\text { Full-matrix } \\
\text { least-squares of } F^{2}\end{array}$ \\
\hline $\mathrm{R}(F)^{\mathrm{a}}$ & 0.0429 & 0.0321 & 0.042 & 0.0219 \\
\hline $\mathrm{R}_{w}\left(F^{2}\right)^{\mathrm{b}}$ & 0.0959 & 0.0711 & 0.0842 & 0.0443 \\
\hline $\mathrm{GOF}_{\mathrm{w}} \mathrm{c}$ & 0.960 & 1.035 & 0.953 & 1.025 \\
\hline $\begin{array}{l}\text { Largest Diff Peak and } \\
\text { Hole }\left(\mathrm{e}^{-} / \AA^{3}\right)\end{array}$ & 5.752 and -3.996 & 2.586 and -2.749 & 1.625 and -1.162 & 1.517 and -0.958 \\
\hline
\end{tabular}


Table S4. Crystallographic Data for Nitride1

\begin{tabular}{|c|c|}
\hline & Nitride1 \\
\hline Molecular Formula & $\mathrm{C}_{72} \mathrm{H}_{90} \mathrm{~N}_{2} \mathrm{O}_{6} \mathrm{Si}_{6} \mathrm{~W}_{2} \cdot\left(\mathrm{C}_{6} \mathrm{H}_{6}\right)$ \\
\hline $\mathrm{FW}_{W}$ & 1693.84 \\
\hline Temp $(\mathrm{K})$ & $90.0(5)$ \\
\hline Crystal System & Monoclinic \\
\hline Space Group & $\mathrm{C}_{2} / \mathrm{c}$ \\
\hline Cell Constants & \\
\hline$a(\AA)$ & $19.956(3)$ \\
\hline$b(\AA)$ & $12.6800(17)$ \\
\hline$c(\AA)$ & $29.462(4)$ \\
\hline$\alpha(\mathrm{deg})$ & 90 \\
\hline$\beta(\operatorname{deg})$ & $99.791(2)$ \\
\hline$\gamma(\operatorname{deg})$ & 90 \\
\hline$Z$ & 4 \\
\hline$V\left(\AA^{3}\right)$ & $7346.6(17)$ \\
\hline Abs Coeff, $\mu_{\text {calc }}\left(\mathrm{mm}^{-1}\right)$ & 3.280 \\
\hline$\delta_{\text {calc }}\left(\mathrm{g} / \mathrm{cm}^{3}\right)$ & 1.531 \\
\hline $\mathrm{F}(000)\left(\mathrm{e}^{-} / \AA^{3}\right)$ & 3431 \\
\hline Crystal Dimensions (mm) & $0.119 \times 0.083 \times 0.078$ \\
\hline Radiation & $\begin{array}{c}\text { Mo K } \alpha \\
(\lambda=0.71073 \AA)\end{array}$ \\
\hline$h, k, l$ Ranges Collected & $-25 \leq \mathrm{h} \leq 26,-16 \leq \mathrm{k} \leq 16,-38 \leq 1 \leq 38$ \\
\hline$\theta$ Range (deg) & $1.91-27.97$ \\
\hline No. of Reflections Collected & 58838 \\
\hline No. of Unique Reflections & 8803 \\
\hline No. of Parameters & 432 \\
\hline Data/Parameter Ratio & 20.4 \\
\hline Refinement Method & $\begin{array}{c}\text { Full-matrix } \\
\text { least-squares of } F^{2}\end{array}$ \\
\hline $\mathrm{R}(F)^{\mathrm{a}}$ & 0.0577 \\
\hline $\mathrm{R}_{w}\left(F^{2}\right)^{\mathrm{b}}$ & 0.1010 \\
\hline $\mathrm{GOF}_{\mathrm{w}} \mathrm{c}$ & 0.9685 \\
\hline Largest Diff Peak and Hole $\left(\mathrm{e}^{-} / \AA^{3}\right)$ & 6.6528 and -4.3272 \\
\hline
\end{tabular}




\section{$\mathrm{C}_{6} \mathrm{H}_{3}\left(\mathrm{C}_{6} \mathrm{H}_{4} \mathrm{SiEt}_{2} \mathrm{OH}\right)_{3}\left(\mathrm{SiP}^{\mathrm{Et}}\right)$}
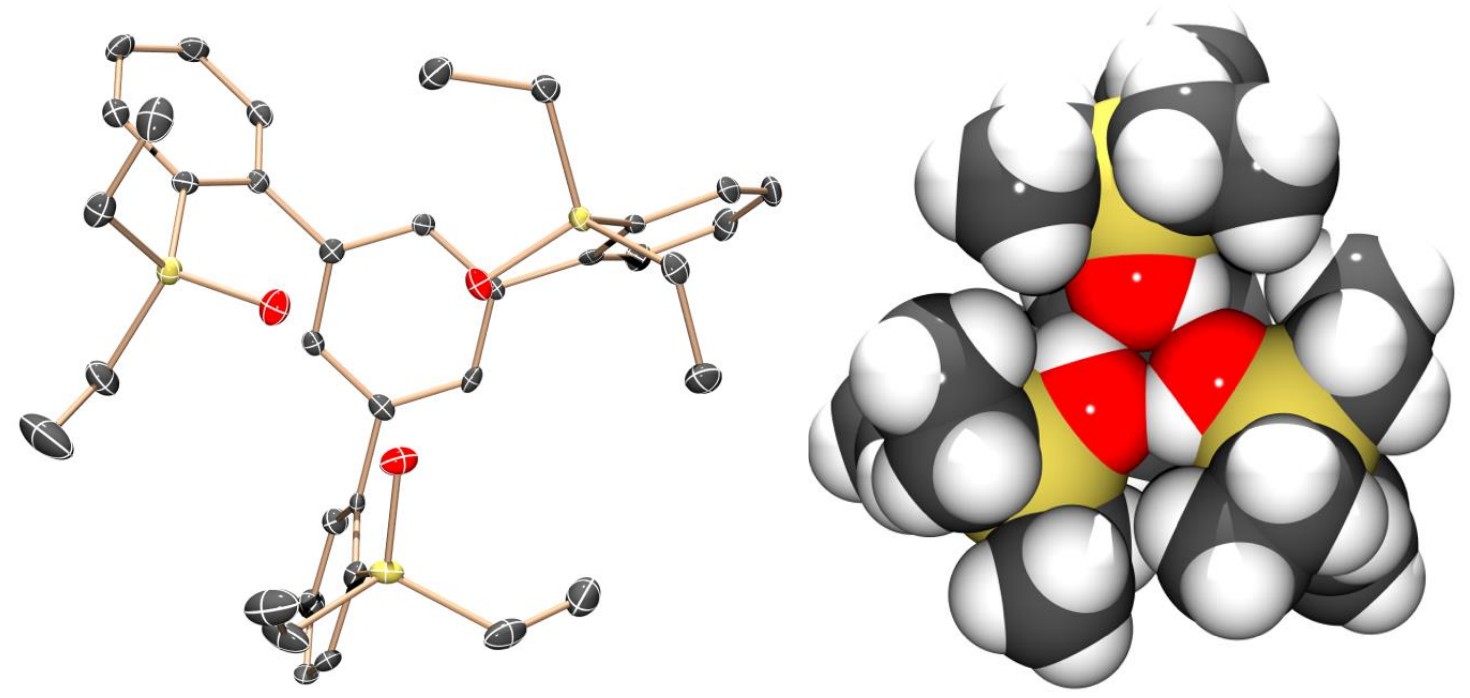

Figure S44. Crystal structure of SiPEt (left) with the other independent molecule removed for clarity, ellipsoids for non-hydrogen atoms are shown at the $50 \%$ probability level. Space-filling model of $\mathbf{S i P}^{\mathrm{Et}}$ (right).

\section{$\left[\mathrm{C}_{6} \mathrm{H}_{3}\left(\mathrm{C}_{6} \mathrm{H}_{4} \mathrm{SiPh}_{2} \mathrm{O}\right)_{3}\right] \mathrm{W} \equiv \mathrm{CMes}(\mathrm{Cat} 3)$}
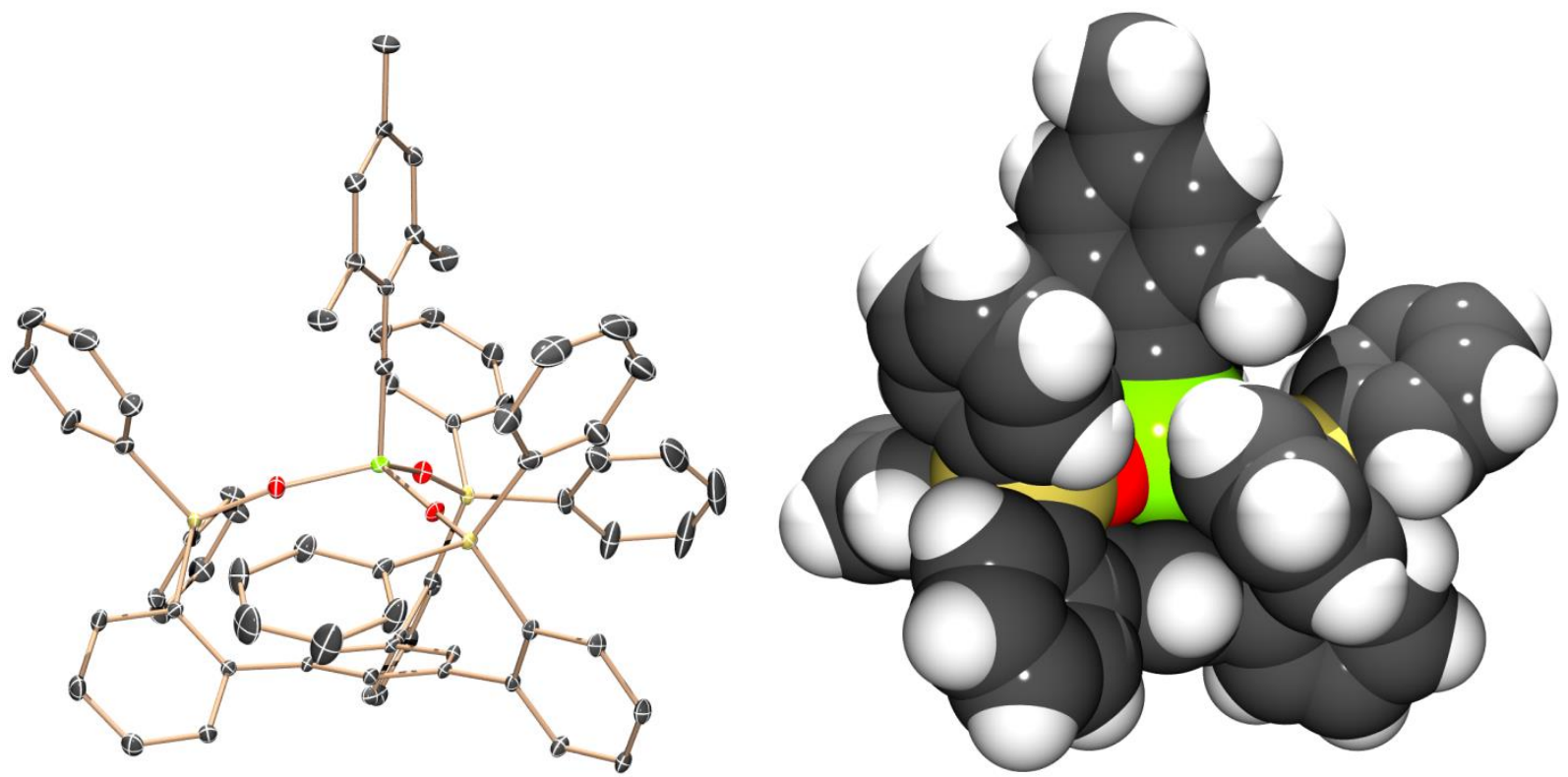

Figure S45. ORTEP of Cat3 (left) with hydrogen atoms omitted for clarity, ellipsoids for the nonhydrogen atoms are shown at the 50\% probability level. Space-filling model of Cat3 (right). 


\section{$\left[\mathrm{C}_{6} \mathrm{H}_{3}\left(\mathrm{C}_{6} \mathrm{H}_{4} \mathrm{SiEt}_{2} \mathrm{O}\right)_{3}\right] \mathrm{Mo} \equiv \mathrm{CMes}(\mathrm{Cat} 4)$}
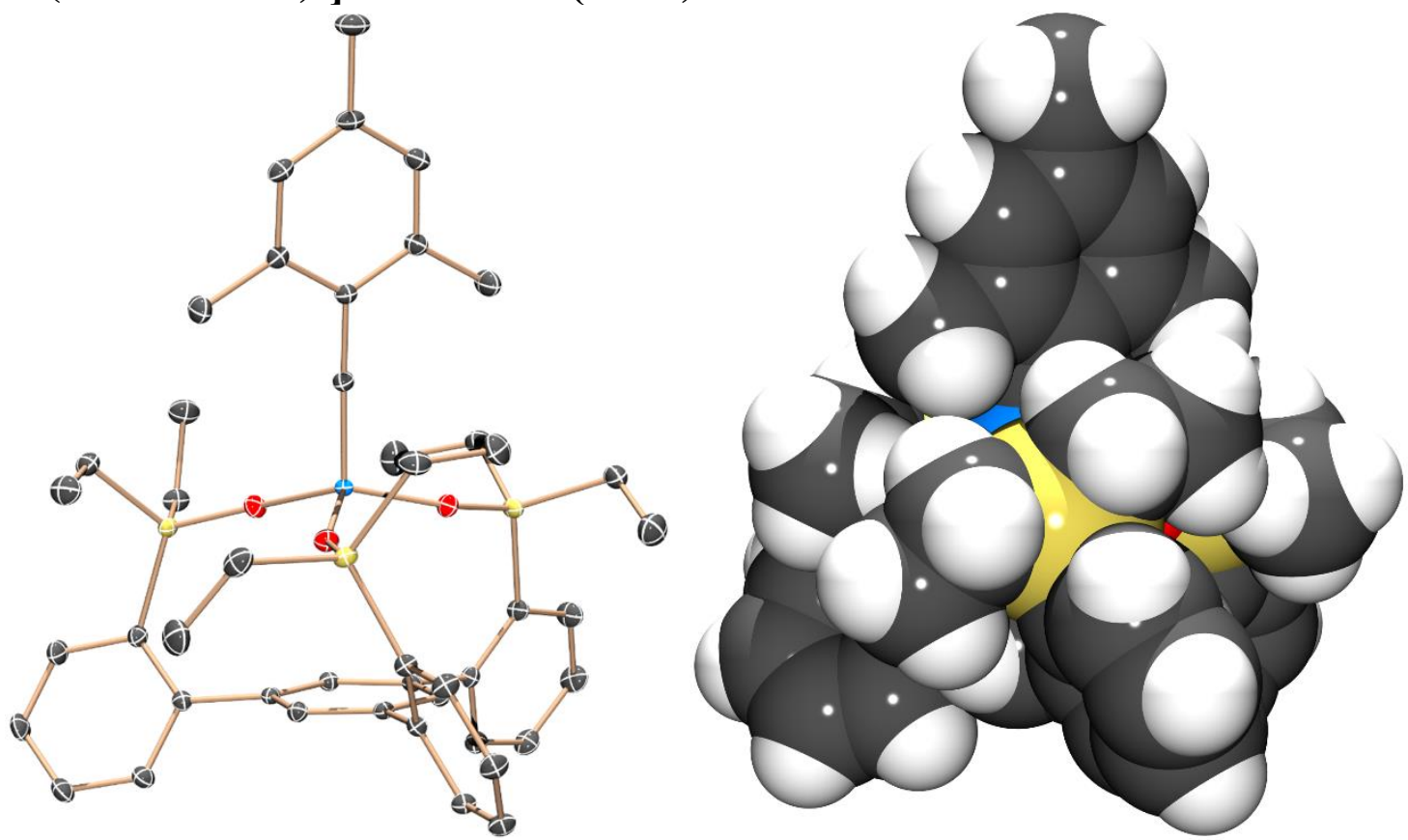

Figure S46. ORTEP of Cat4 (left) with hydrogen atoms omitted for clarity, ellipsoids for the nonhydrogen atoms are shown at the 50\% probability level. Space-filling model of Cat4 (right).

\section{$\left[\mathrm{C}_{6} \mathrm{H}_{3}\left(\mathrm{C}_{6} \mathrm{H}_{4} \mathrm{SiEt}_{2} \mathrm{O}\right)_{3}\right] \mathrm{W} \equiv \mathrm{CMes}(\mathrm{Cat5})$}
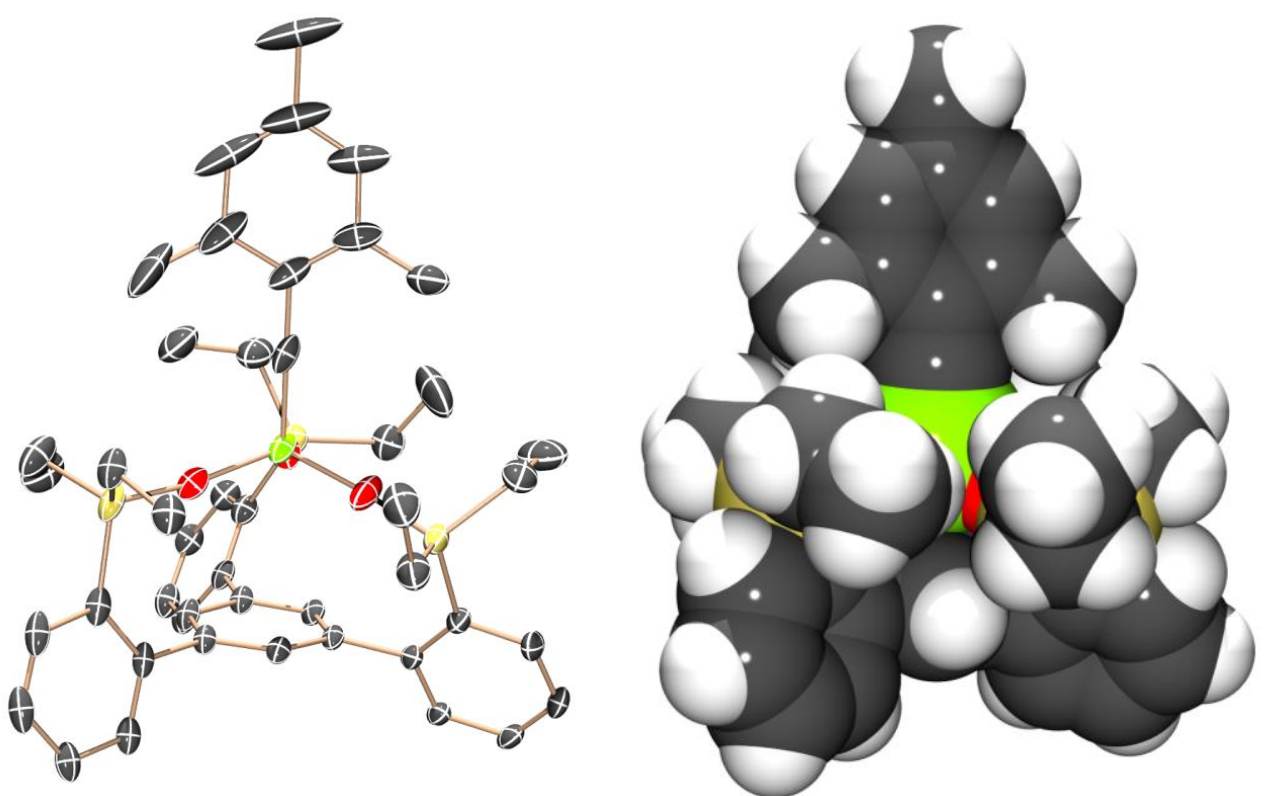

Figure S47. ORTEP of Cat5 (left) with the other independent molecule, hydrogen atoms, and solvent omitted for clarity, ellipsoids for the non-hydrogen atoms are shown at the 50\% probability level. Space-filling model of Cat5 (right). 

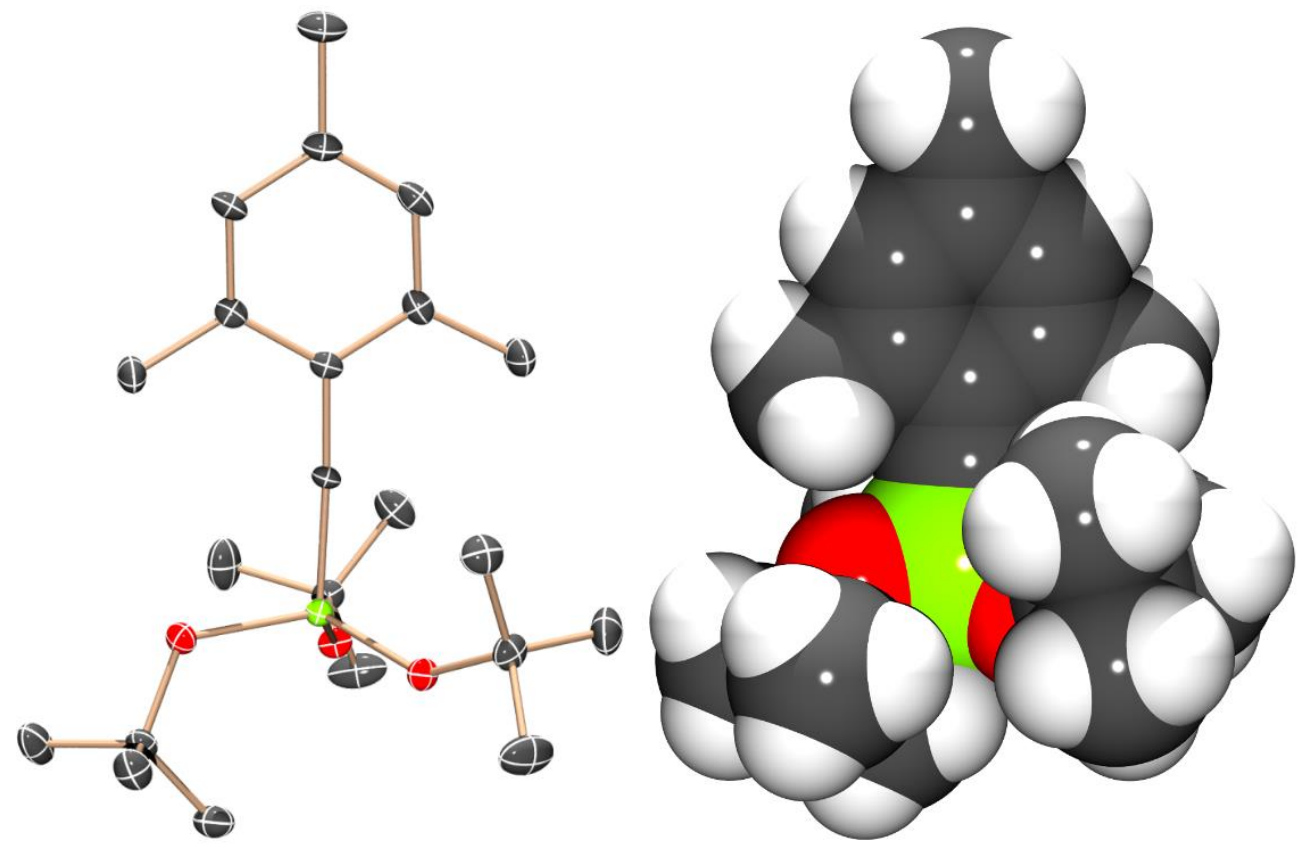

Figure S48. Crystal structure of Pre2 (left) with hydrogen atoms removed for clarity, ellipsoids for non-hydrogen atoms are shown at the 50\% probability level. Space-filling model of Pre2 (right).

\section{$\left[\mathrm{C}_{6} \mathrm{H}_{3}\left(\mathrm{C}_{6} \mathrm{H}_{4} \mathrm{SiPh}_{2} \mathrm{O}\right)_{3}\right] \mathrm{Mo}\left(\mathrm{C}_{3} \mathrm{Bu}_{3}\right)\left(\mathrm{MT}_{\mathrm{d}} 2\right)$}
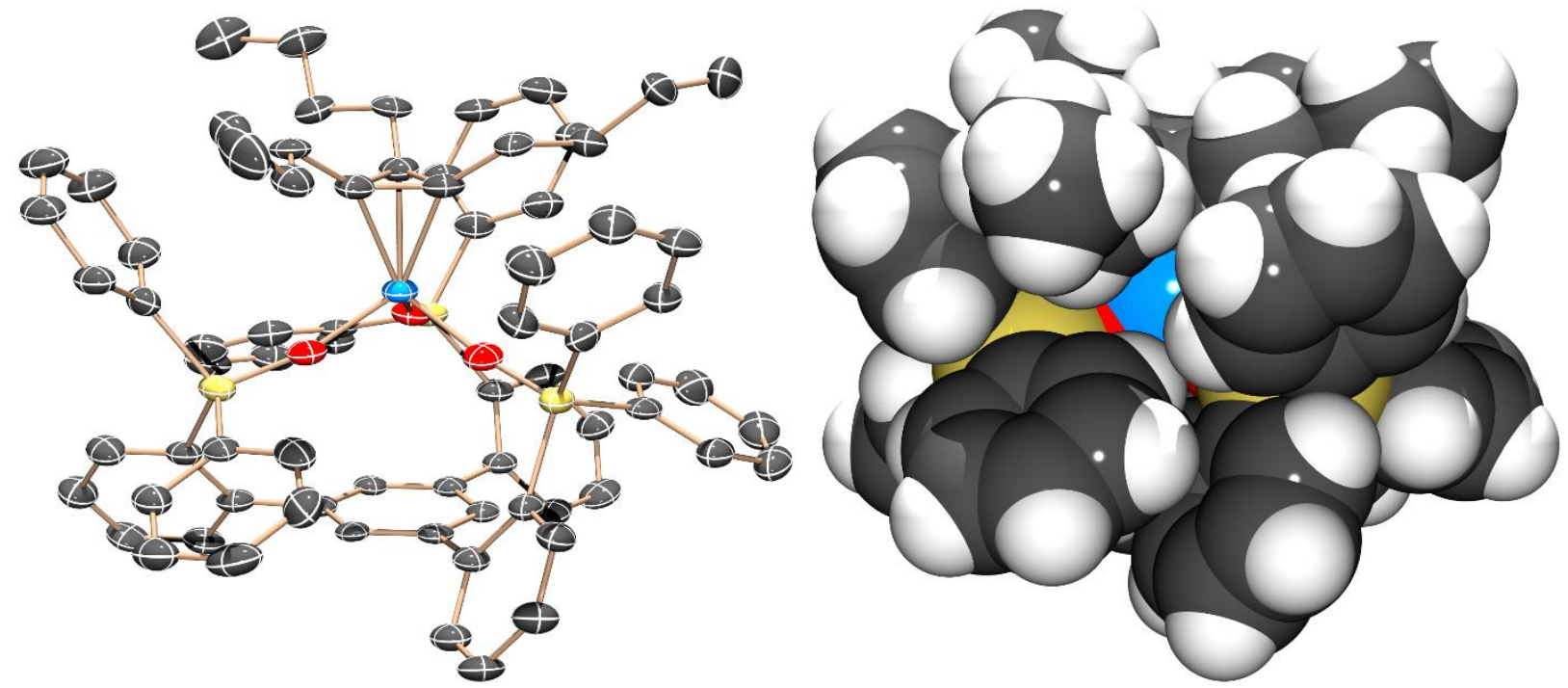

Figure S49. ORTEP of MTd2 (left) with hydrogen atoms omitted for clarity, ellipsoids for the non-hydrogen atoms are shown at the 50\% probability level. Space-filling model of $\mathbf{M T}_{\mathrm{d}} \mathbf{2}$ (right). 


\section{$\left.\left[\mathrm{C}_{6} \mathrm{H}_{3}\left(\mathrm{C}_{6} \mathrm{H}_{4} \mathrm{SiPh}_{2} \mathrm{O}\right)_{3}\right] \mathrm{W}\left(\mathrm{C}_{3} \mathrm{ArEt}\right)_{2}\right)(\mathrm{MCBD} 2)$}
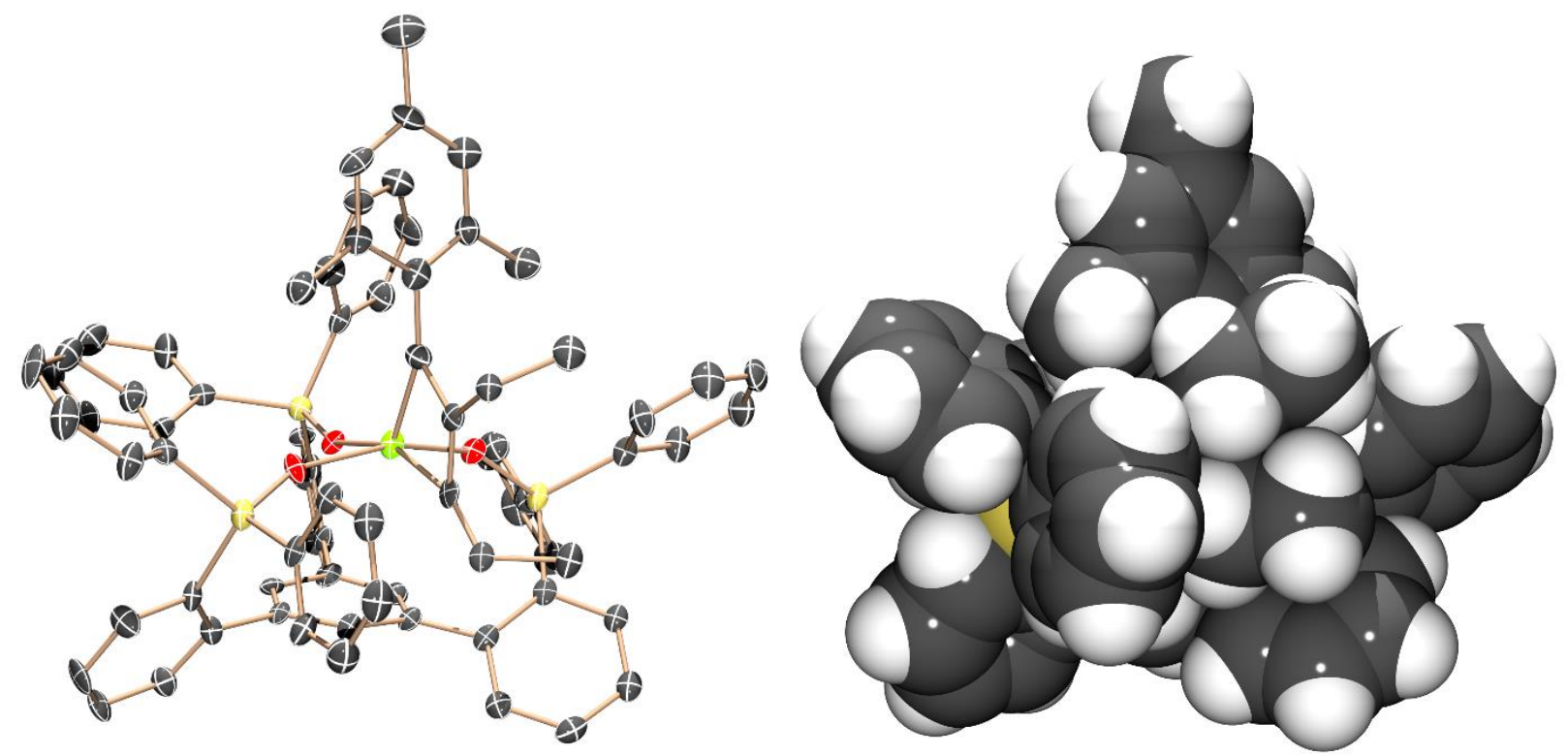

Figure S50. ORTEP of MCBD2 (left) with hydrogen atoms and solvent omitted for clarity, ellipsoids for the non-hydrogen atoms are shown at the 50\% probability level. Space-filling model of MCBD2 (right).

\section{$\left[\mathrm{C}_{6} \mathrm{H}_{3}\left(\mathrm{C}_{6} \mathrm{H}_{4} \mathrm{SiEt}_{2} \mathrm{O}\right)_{3}\right] \mathrm{W}\left(\mathrm{C}_{3} \mathrm{ArEt}_{2}\right)(\mathrm{MCBD3})$}
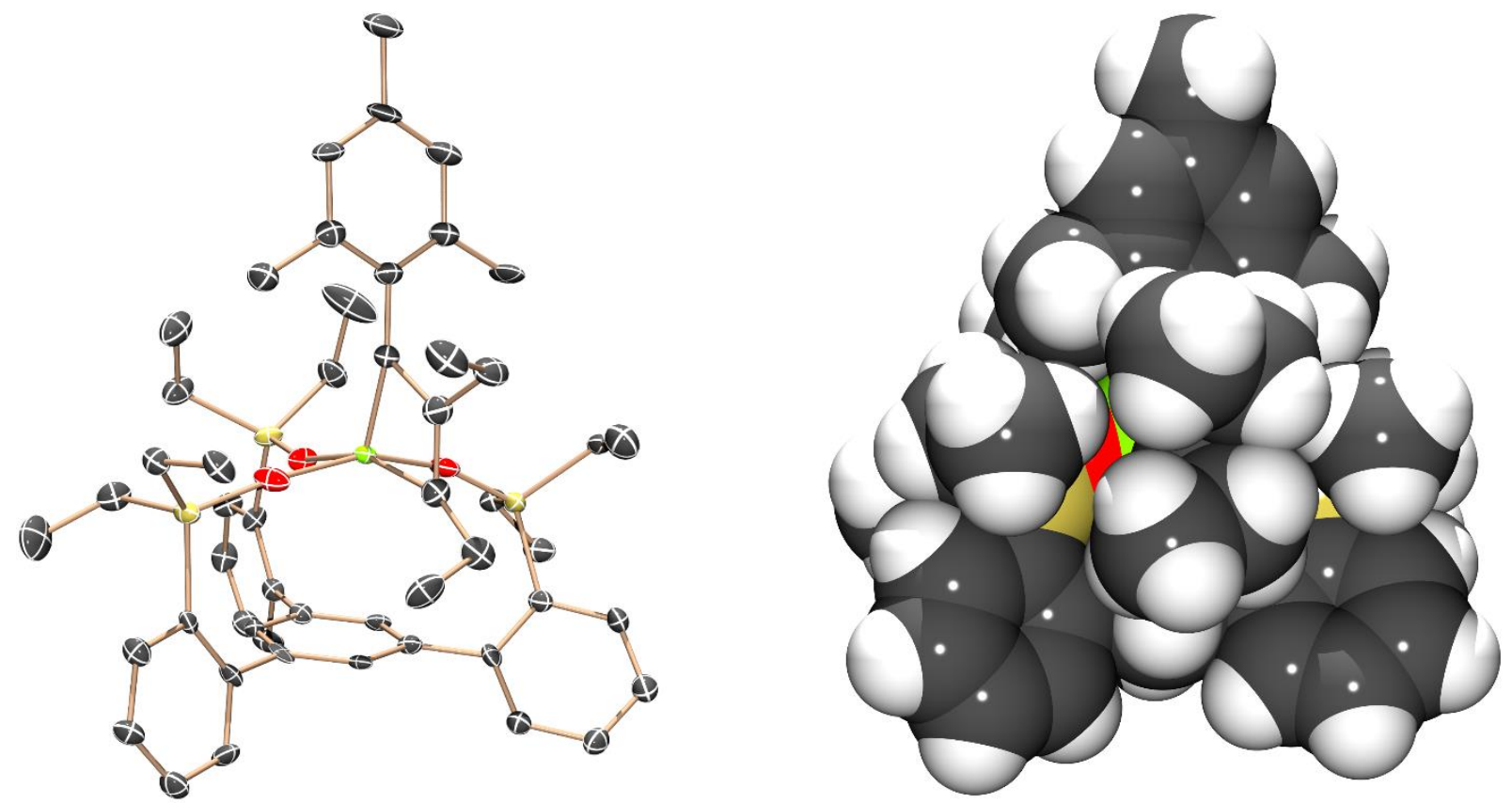

Figure S51. ORTEP of MCBD3 (left) with hydrogen atoms, solvent and the other independent molecule omitted for clarity. Ellipsoids for the non-hydrogen atoms are shown at the 50\% probability level. Space-filling model of MCBD3 (right). 


\section{$\left[\mathrm{C}_{6} \mathrm{H}_{3}\left(\mathrm{C}_{6} \mathrm{H}_{4} \mathrm{SiPh}_{2} \mathrm{O}\right)_{3}\right] \mathrm{W}\left(\mathrm{C}_{3} \mathrm{Et}_{3}\right)(\mathrm{MCBD} 4)$}
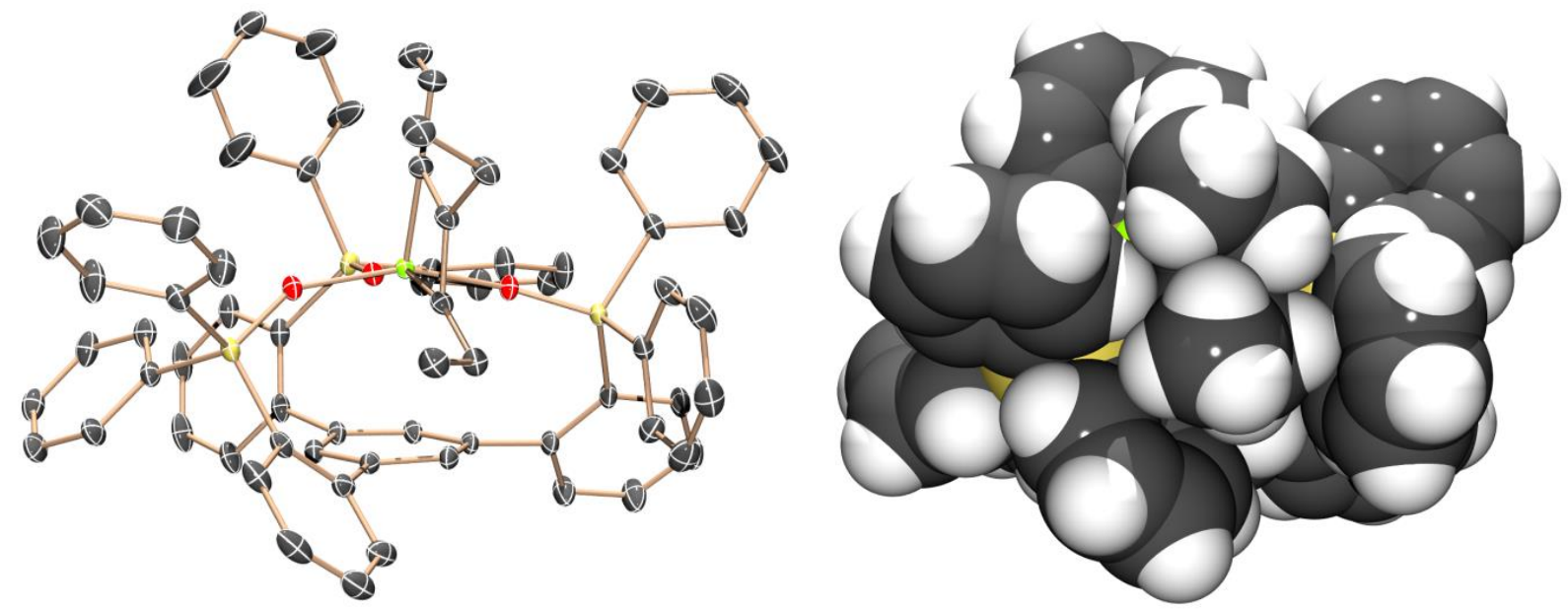

Figure S52. ORTEP of MCBD4 (left) with hydrogen atoms and solvent omitted for clarity, ellipsoids for the non-hydrogen atoms are shown at the 50\% probability level. Space-filling model of MCBD4 (right).

\section{$\left[\mathrm{C}_{6} \mathrm{H}_{3}\left(\mathrm{C}_{6} \mathrm{H}_{4} \mathrm{SiEt}_{2} \mathrm{O}\right)_{3}\right] \mathrm{W}\left(\mathrm{C}_{3} \mathrm{Et}_{3}\right)(\mathrm{MCBD5})$}
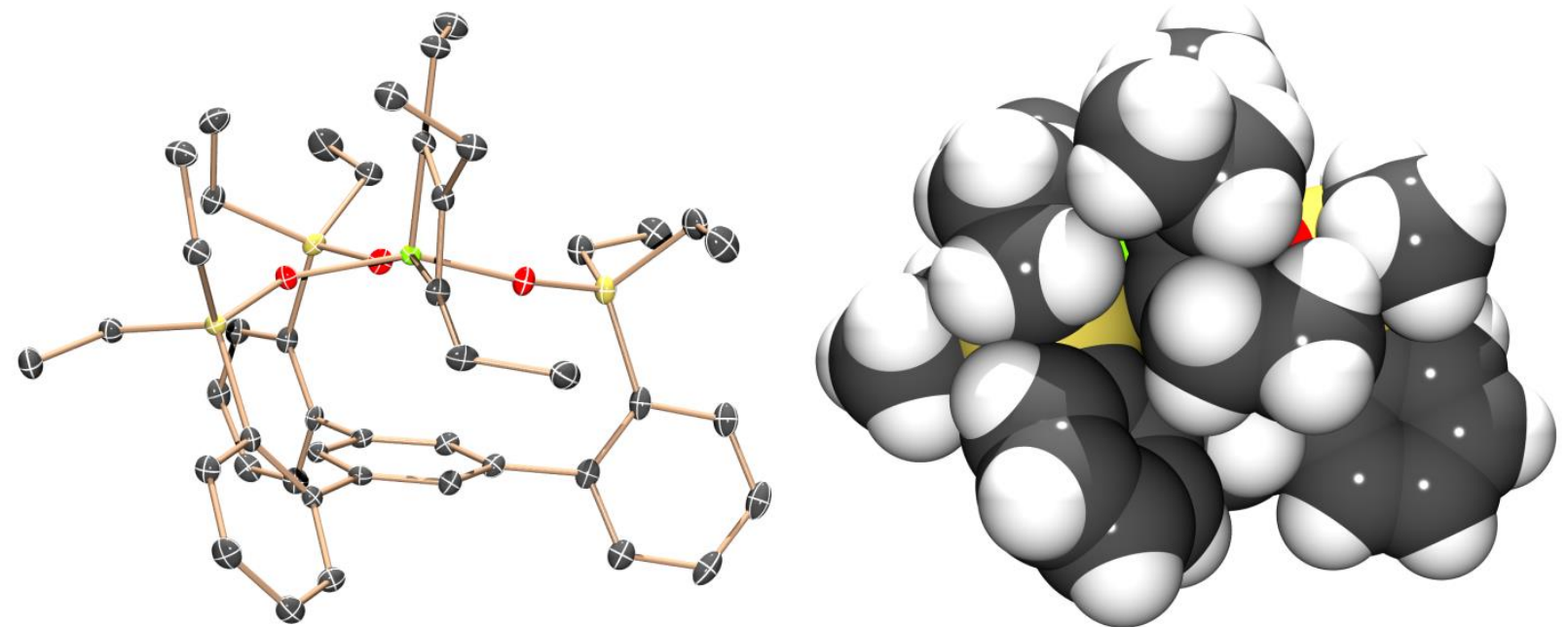

Figure S53. ORTEP of MCBD5 (left) with hydrogen atoms omitted for clarity, ellipsoids for the non-hydrogen atoms are shown at the 50\% probability level. Space-filling model of MCBD5 (right). 


\section{$\left[\mathrm{C}_{6} \mathrm{H}_{3}\left(\mathrm{C}_{6} \mathrm{H}_{4} \mathrm{SiEt}_{2} \mathrm{O}\right)_{3}\right] \mathrm{W}\left(\mathrm{C}_{3} \mathrm{ArAr}{ }^{\prime} \mathrm{Me}\right)(\mathrm{MCBD6})$}
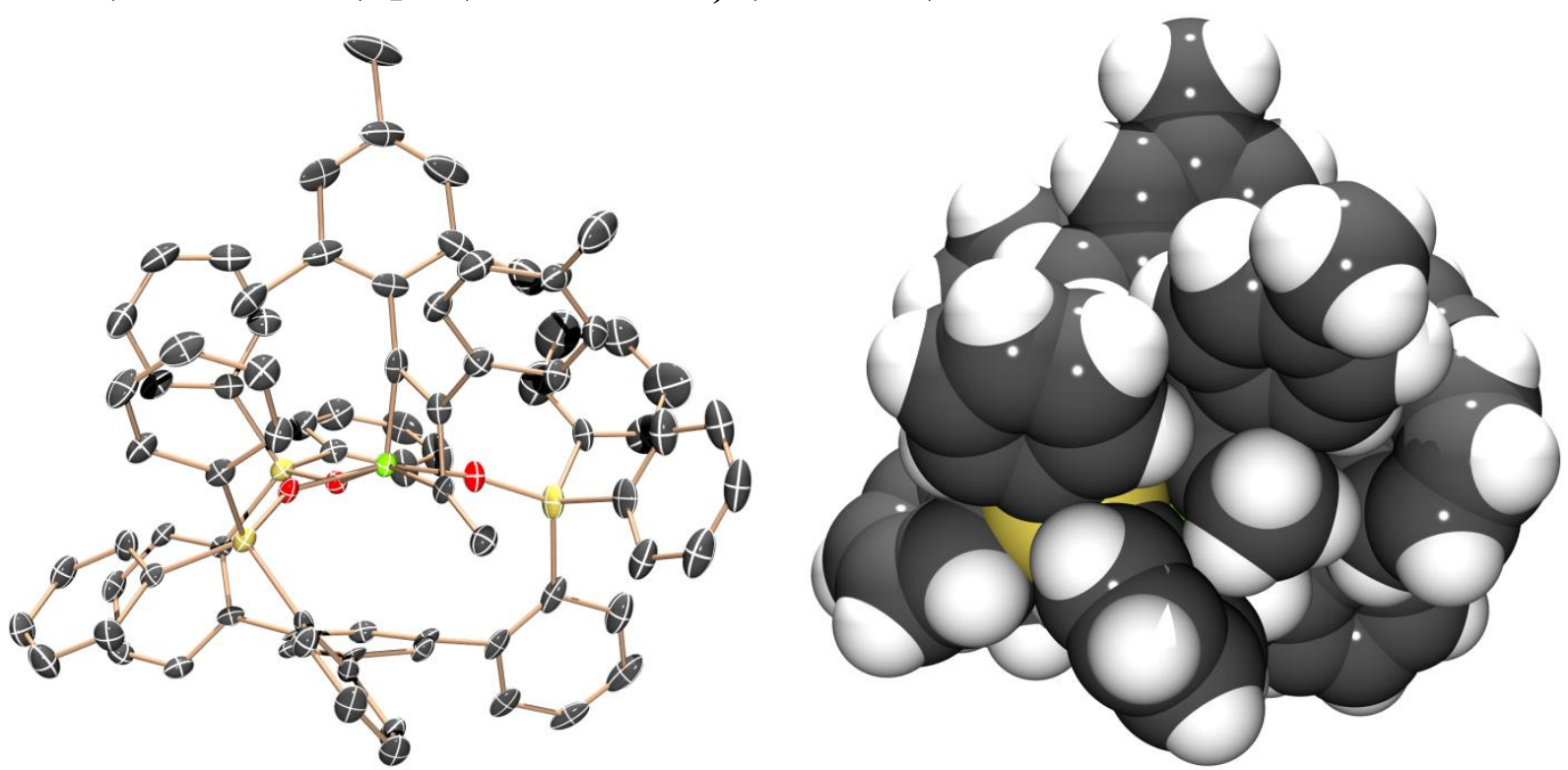

Figure S54. ORTEP of MCBD6 (left) with hydrogen atoms omitted for clarity, ellipsoids for the non-hydrogen atoms are shown at the 50\% probability level. Space-filling model of MCBD6 (right).

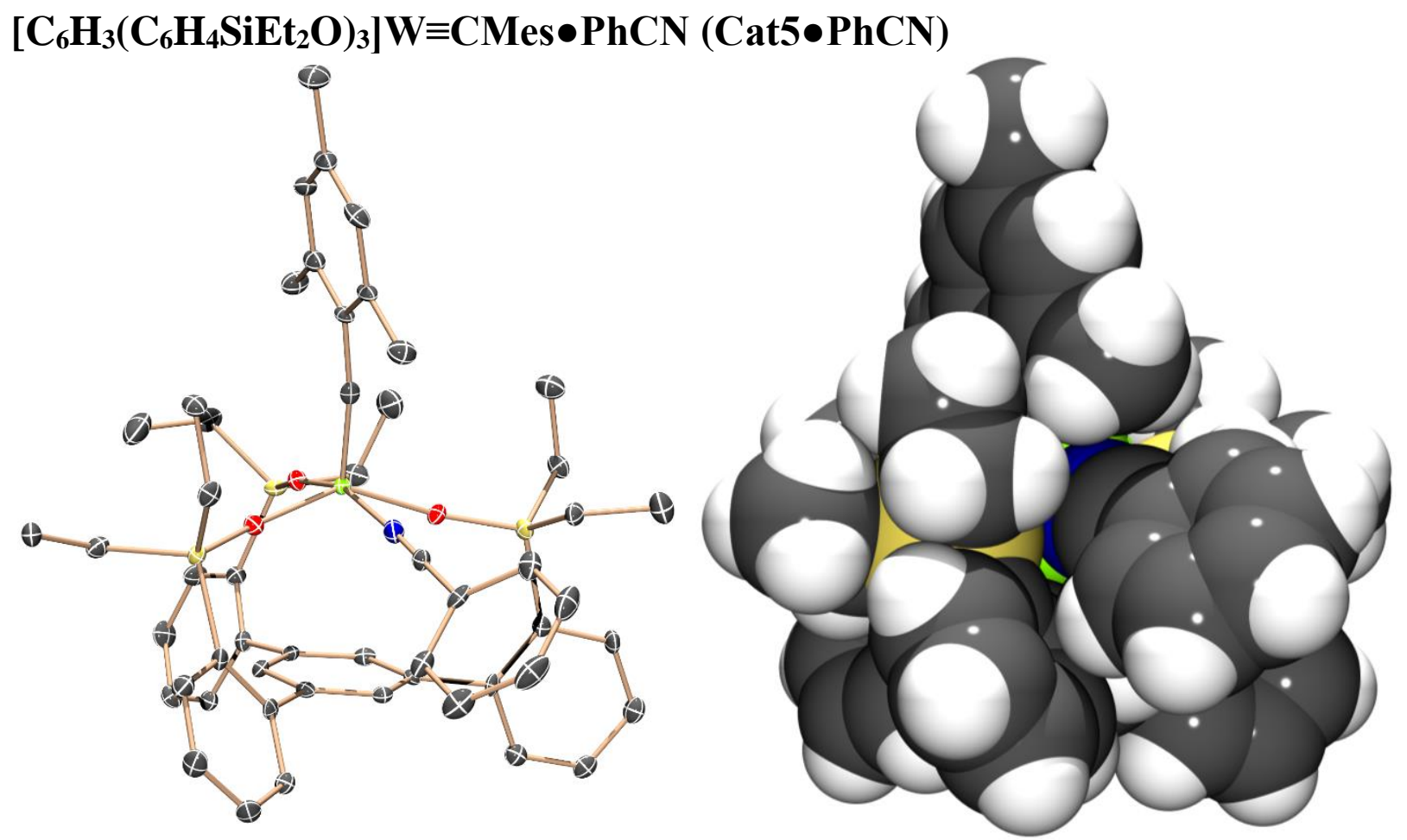

Figure S55. ORTEP of Cat5•PhCN (left) with hydrogen atoms omitted for clarity, ellipsoids for the non-hydrogen atoms are shown at the $50 \%$ probability level. Space-filling model of Cat5•PhCN (right). 


\section{$\left(\left[\mathrm{C}_{6} \mathrm{H}_{3}\left(\mathrm{C}_{6} \mathrm{H}_{4} \mathrm{SiEt}_{2} \mathrm{O}\right)_{3}\right] \mathrm{W} \equiv \mathrm{N}\right)_{2}$ (Nitride1)}
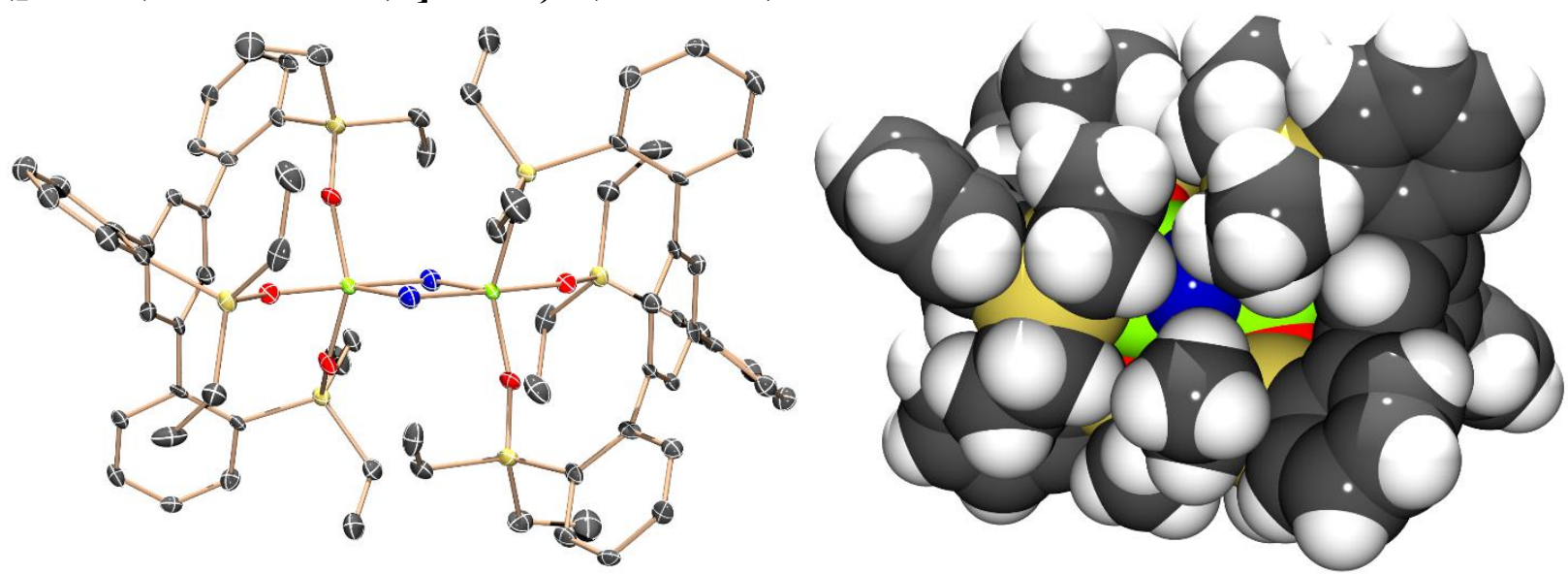

Figure S56. ORTEP of Nitride1 (left) with hydrogen atoms and solvent omitted for clarity, ellipsoids for the non-hydrogen atoms are shown at the 50\% probability level. Space-filling model of Nitride1(right).

\section{Computational details}

\section{General Remarks}

All optimizations of intermediates and transition states were calculated using restricted B3LYPD3/def2SVP-LANL2DZ(M) level of the theory ${ }^{10}$ in implicit solvent (benzene) using CPCM as solvation model ${ }^{11}$ as implemented in Gaussian09. Frequency calculations, at the same level of theory, were used to obtain thermal corrections (at 298K) and to characterize optimized structures as transition states (only a single imaginary frequency) or intermediate (if no imaginary frequencies were found). Intrinsic reaction coordinate (IRCs) calculations were undertaken to ensure transition states connected illustrated ground states. Single point energy calculations using B3LYP-D3/def2TZVP-SDD(M) with solvent corrections calculated in implicit solvent (benzene) using CPCM were also performed on all structures. ${ }^{12}$ For comparison, single point energy calculations with restricted PBEPBE/def2TZVP-SDD(M) were calculated with solvent corrections calculated in implicit solvent (benzene) using CPCM as a solvation model. All reported charges are from Mulliken population analysis. ${ }^{13}$ All 3-D structures were generated using CYLview. ${ }^{14}$ Noncovalent interaction (NCI) analysis, also known as reduce density gradient (RDG) method, was performed on Multiwfn to study the possible effect of noncovalent interaction in the metallatetrahedrane intermediates. ${ }^{15}$ Extension distance of 0 Bohr, medium quality grid (totally about 512000 points) were set by default. Further visualization of the color-filled RDG isosurface was realized by VMD, where RDG isosurface and color range were set as 0.5 , and -0.035 to 0.2 , respectively. ${ }^{16}$ The energy decomposition analysis calculations were performed using the secondgeneration absolutely localized molecular orbitals ${ }^{17}$ (ALMO-EDA) method implemented in QChem 5.0. ${ }^{18}$ The HF/6-311G(d,p) method was used as employed by Liu. ${ }^{19}$ This method decomposes the through-space interaction energies between the ligand and substrate into the energetic components including the Pauli repulsion energy $\left(\Delta \mathrm{E}_{\text {Pauli }}\right)$, the electrostatic energy $\left(\Delta \mathrm{E}_{\text {elstat }}\right)$, the polarization energy $\left(\Delta \mathrm{E}_{\mathrm{pol}}\right)$ and the charge transfer energy $\left(\Delta \mathrm{E}_{\mathrm{ct}}\right)$ Distortion energies $\left(\Delta \mathrm{E}_{\text {dist }}\right)$, or the energy required to distort the geometry of the starting intermediate to the transition 
state geometry as described by the distortion-interaction model, ${ }^{20}$ were calculated using the B3LYP-D3/defTZVP-SDD(M)-CPCM(benzene)//B3LYP-D3/def2SVP-LANL2DZ(M)-

CPCM(benzene)level of theory in Gaussian 09.

\section{Full Reference of Gaussian 09 Software}

Gaussian 09, Revision E.01, M. J. Frisch, G. W. Trucks, H. B. Schlegel, G. E. Scuseria, M. A. Robb, J. R. Cheeseman, G. Scalmani, V. Barone, B. Mennucci, G. A. Petersson, H. Nakatsuji, M. Caricato, X. Li, H. P. Hratchian, A. F. Izmaylov, J. Bloino, G. Zheng, J. L. Sonnenberg, M. Hada, M. Ehara, K. Toyota, R. Fukuda, J. Hasegawa, M. Ishida, T. Nakajima, Y. Honda, O. Kitao, H. Nakai, T. Vreven, J. A. Montgomery, Jr., J. E. Peralta, F. Ogliaro, M. Bearpark, J. J. Heyd, E. Brothers, K. N. Kudin, V. N. Staroverov, R. Kobayashi, J. Normand, K. Raghavachari, A. Rendell, J. C. Burant, S. S. Iyengar, J. Tomasi, M. Cossi, N. Rega, J. M. Millam, M. Klene, J. E. Knox, J. B. Cross, V. Bakken, C. Adamo, J. Jaramillo, R. Gomperts, R. E. Stratmann, O. Yazyev, A. J. Austin, R. Cammi, C. Pomelli, J. W. Ochterski, R. L. Martin, K. Morokuma, V. G. Zakrzewski, G. A. Voth, P. Salvador, J. J. Dannenberg, S. Dapprich, A. D. Daniels, Ö. Farkas, J. B. Foresman, J. V. Ortiz, J. Cioslowski, and D. J. Fox, Gaussian, Inc., Wallingford CT, 2009.

\section{Choice of Computational Method}

The computational method of B3LYP-D3/def2TZVP-SDD(M)-CPCM(benzene)//B3LYPD3/def2SVP-LANL2DZ(M)-CPCM(benzene) was chosen as it provides an excellent balance between accuracy and computational cost, all while reproducing structural features from x-ray crystal structures. Pseudopotentials such as LANL2DZ and SDD are very commonly used on the metal center to reduce computational cost while maintaining the accuracy of the calculation. ${ }^{21}$ Furthermore, pseudopotentials such as used LANL2DZ and SDD have been shown to be effective in describing Mo and $\mathrm{W}$ compounds. ${ }^{22}$ 

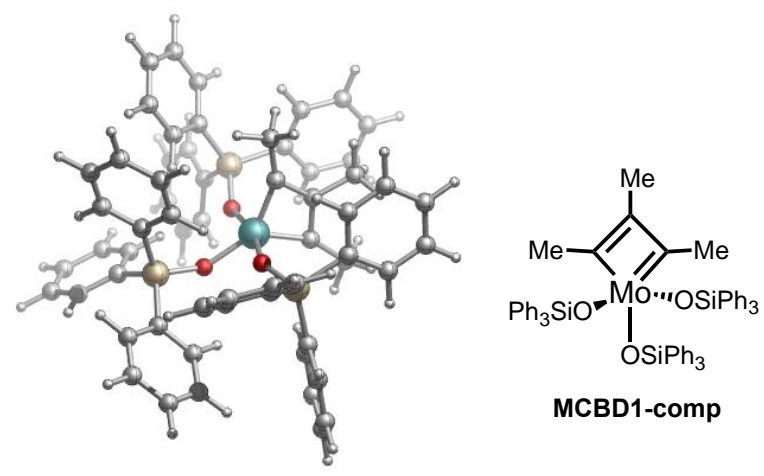

\begin{tabular}{|c|c|}
\hline & MCBD1-comp \\
\hline Mo1-C1 & 1.920 \\
\hline Mo-O Avg & 1.991 \\
\hline O-Si $\mathbf{A v g}_{\mathrm{Avg}}$ & 1.653 \\
\hline C1-C2 & 1.426 \\
\hline $\mathrm{C} 2-\mathrm{C} 3$ & 1.466 \\
\hline C3-Mo1 & 1.889 \\
\hline Mo1-C1-C2 & 79.18 \\
\hline C1-C2-C3 & 119.51 \\
\hline C2-C3-Mo1 & 79.32 \\
\hline C3-Mo1-C1 & 81.98 \\
\hline Mo-O-Si Avg & 143.71 \\
\hline
\end{tabular}

Table S5. List of Bond lengths and angles for MCBD1-comp, optimized using B3LYPD3/def2SVP-LANL2DZ(Mo)-CPCM(benzene).
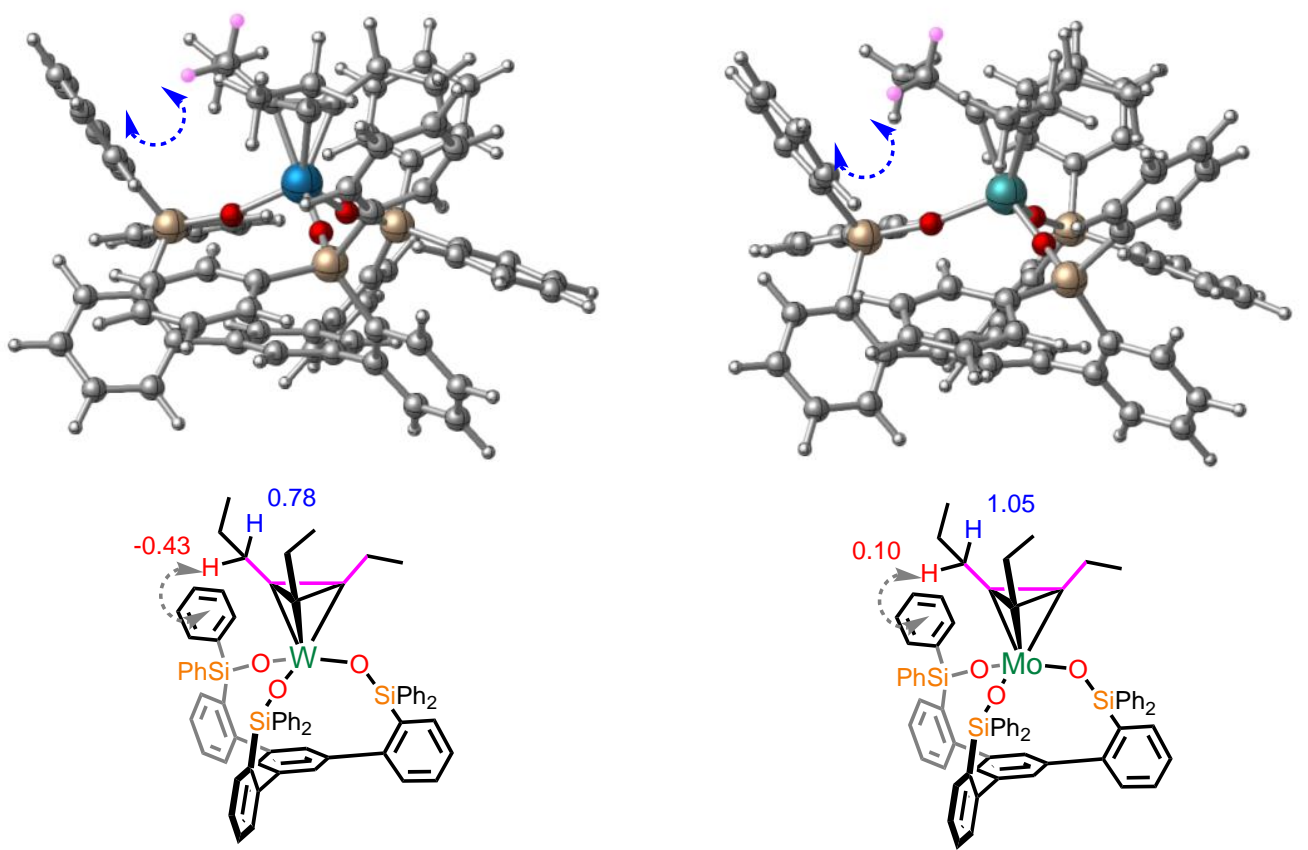

Figure S57. Calculated ${ }^{1} \mathrm{H}(\mathrm{ppm})$ chemical shift for tungsten with $\mathrm{SiP}^{\mathrm{Ph} 6}$ system and molybdenum with $\mathrm{SiP}^{\mathrm{Ph} 6}$ system computed at the B3LYP-D3/def2TZVP-SDD(M)- 
CPCM(benzene)// B3LYP-D3/def2SVP-LANL2DZ(M)-CPCM(benzene)level of theory. The calculated chemical shifts of the shown hydrogen atoms on the ethyl groups provide evidence for the $\mathrm{CH} \cdots \pi$ interaction between the ethyl side chains and the aryl groups on the ligand.

\section{Substrate-Dependence on Reaction Rates}

For comparison of the experimental results of the apparent substrate-dependence on reaction rates, we have computed the lowest energy pathway to product formation for an aryl-propyne substrate with the less sterically hindered SiMe ligand for both tungsten and molybdenum. These pathways are given in Figures S58 and S59 below. While the barrier to [2+2]-cycloaddition is lower in energy for tungsten compared to molybdenum $(9.2 \mathrm{vs}$. $16.6 \mathrm{kcal} / \mathrm{mol})$, it is clear from our computations that the tungsten system would be slower due to the thermodynamically stabilized MCBD intermediate [W]-ent-C' $(-0.3 \mathrm{kcal} / \mathrm{mol})$. From here, the MCBD will undergo retro-[2+2] to yield product, with a relative energy barrier of $12.6 \mathrm{kcal} / \mathrm{mol}$ for tungsten and only $7.0 \mathrm{kcal} / \mathrm{mol}$ for molybdenum. These computations suggest that the reaction rates are dependent on the substrate for tungsten. 


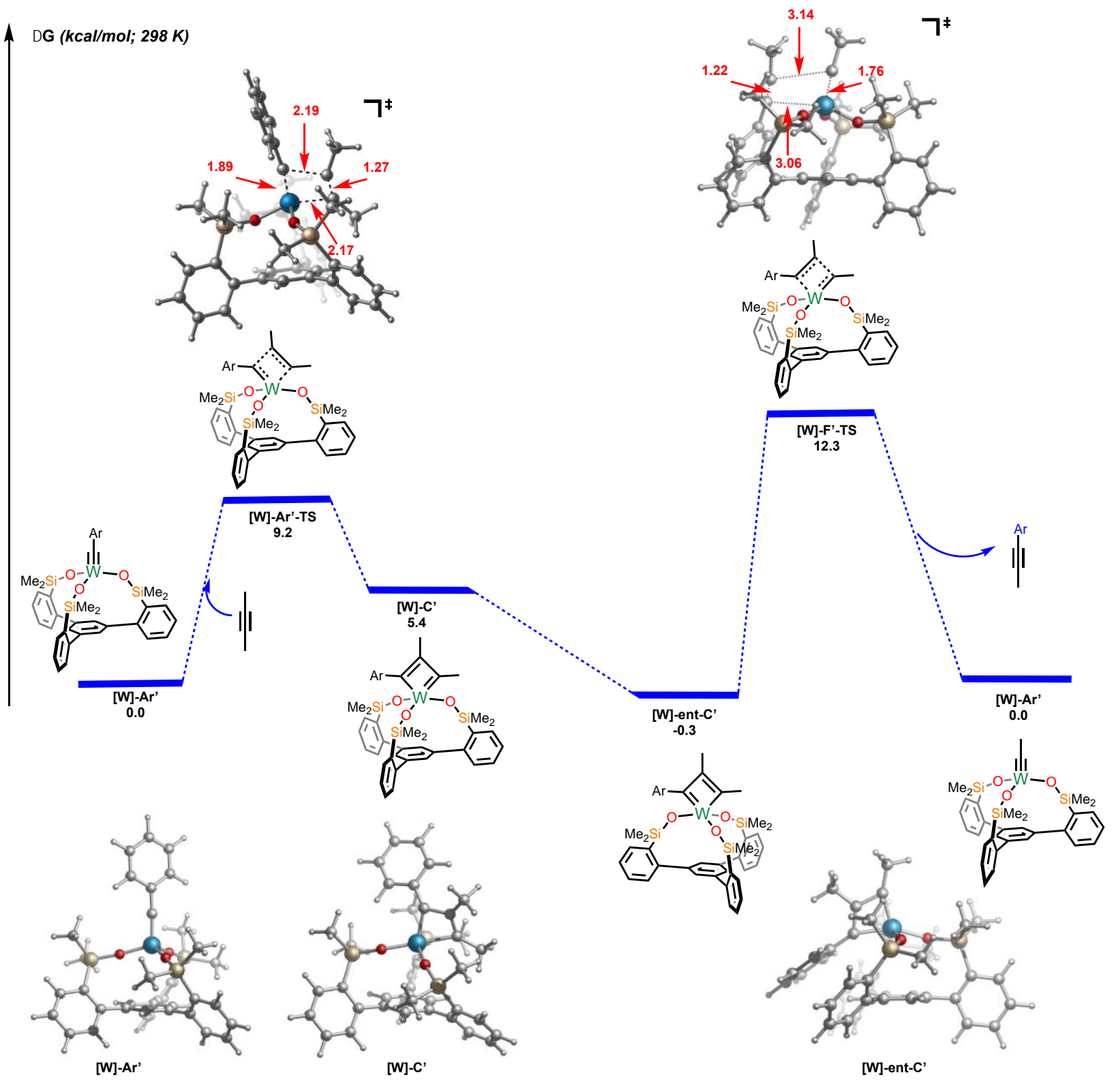

Figure S58. Energetics of MCBD formation via [2+2] cycloaddition for tungsten with SiPMe ligand showing the cross-metathesis of aryl-propyne substrates. Free energies $(\mathrm{kcal} / \mathrm{mol})$ are computed at the B3LYP-D3/def2TZVP-SDD(W)-CPCM(benzene)// B3LYP-D3/def2SVPLANL2DZ(W)-CPCM(benzene)level of theory. 


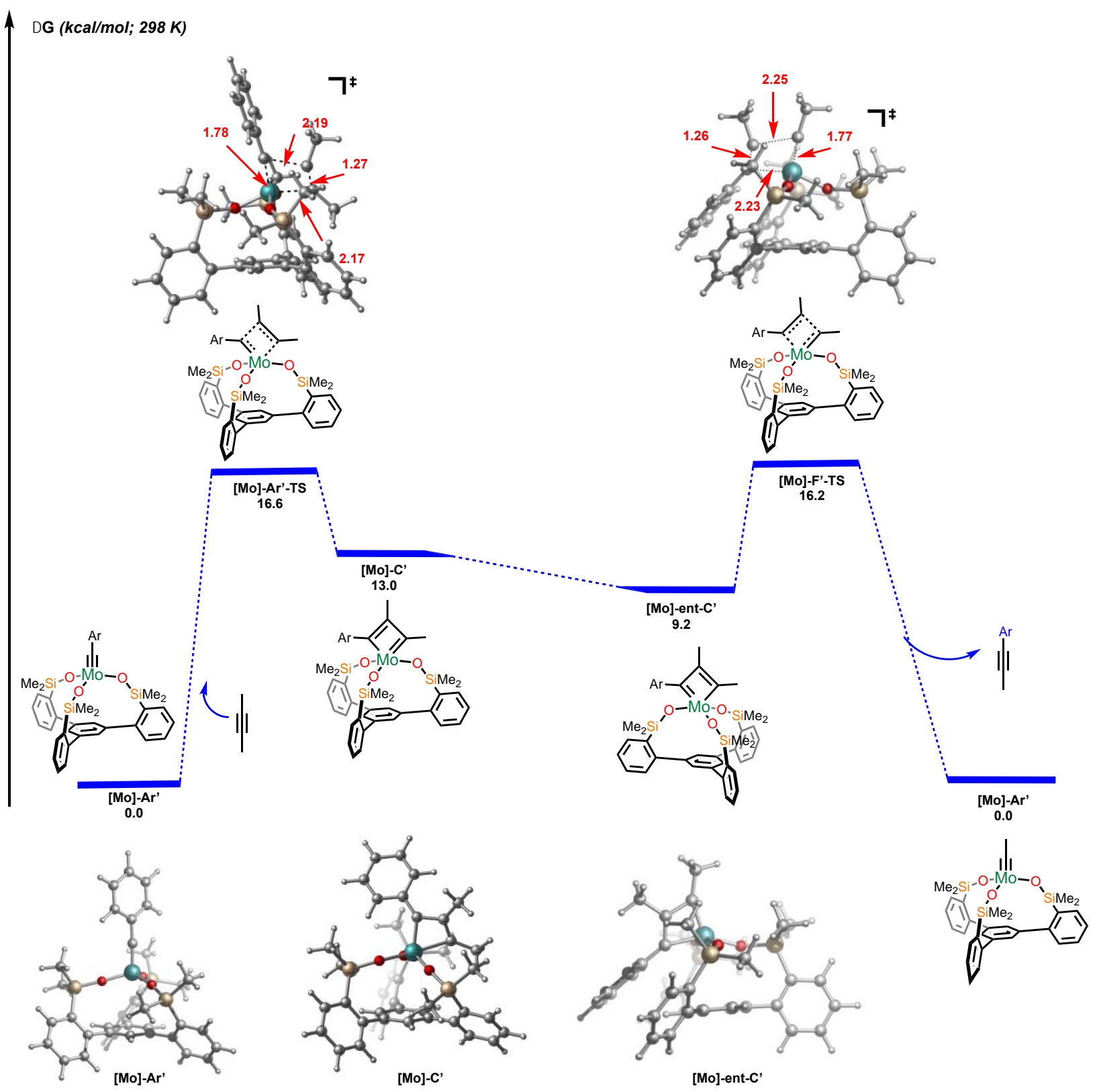

Figure S59. Energetics of MCBD formation via [2+2] cycloaddition for molybdenum with SiPMe ligand showing the cross-metathesis of aryl-propyne substrates. Free energies $(\mathrm{kcal} / \mathrm{mol})$ are computed at the B3LYP-D3/def2TZVP-SDD(Mo)-CPCM(benzene)// B3LYP-D3/def2SVPLANL2DZ(Mo)-CPCM(benzene)level of theory. 


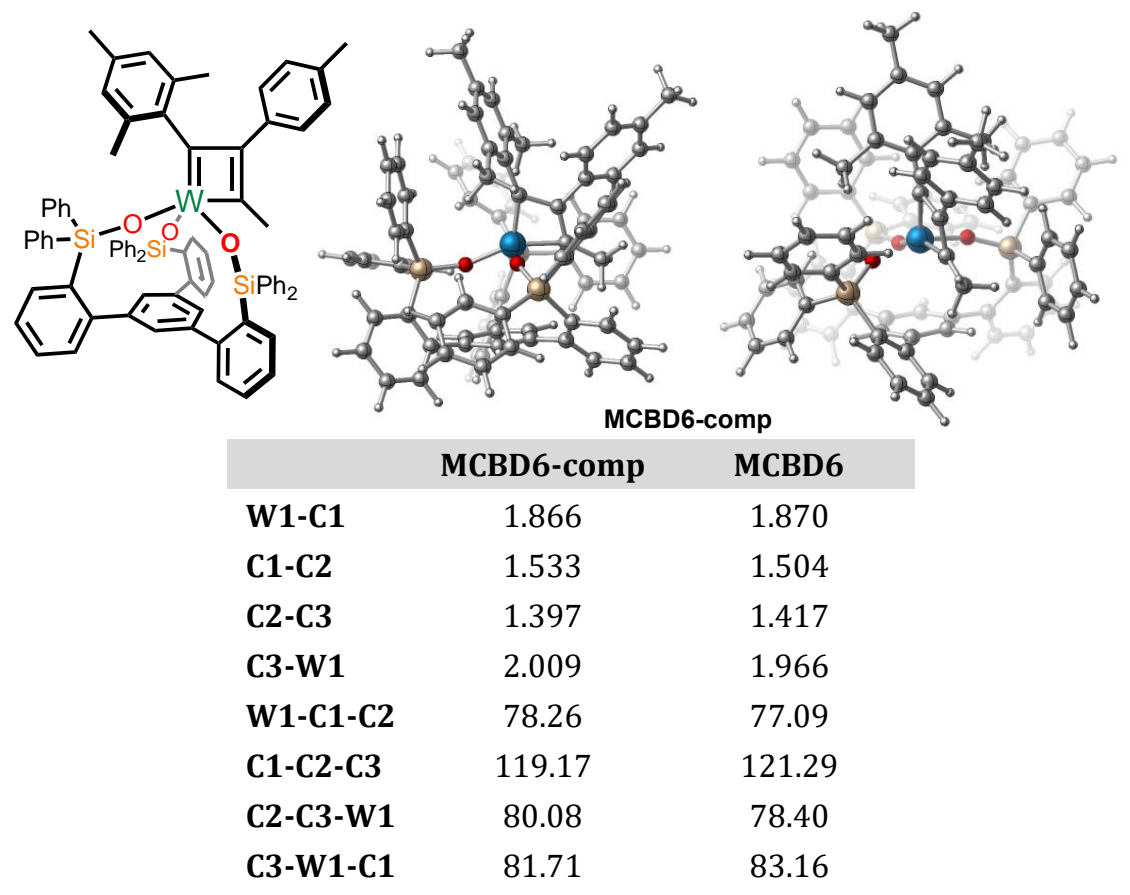

Table S6. List of Bond lengths and angles for MCBD6-comp, optimized using B3LYPD3/def2SVP-LANL2DZ(M)-CPCM(benzene) compared to those of the x-ray crystal structure of MCBD6. Similar to that observed in MCBD6, the tolyl group is pointing away from the basal arene to minimize steric crowding. 


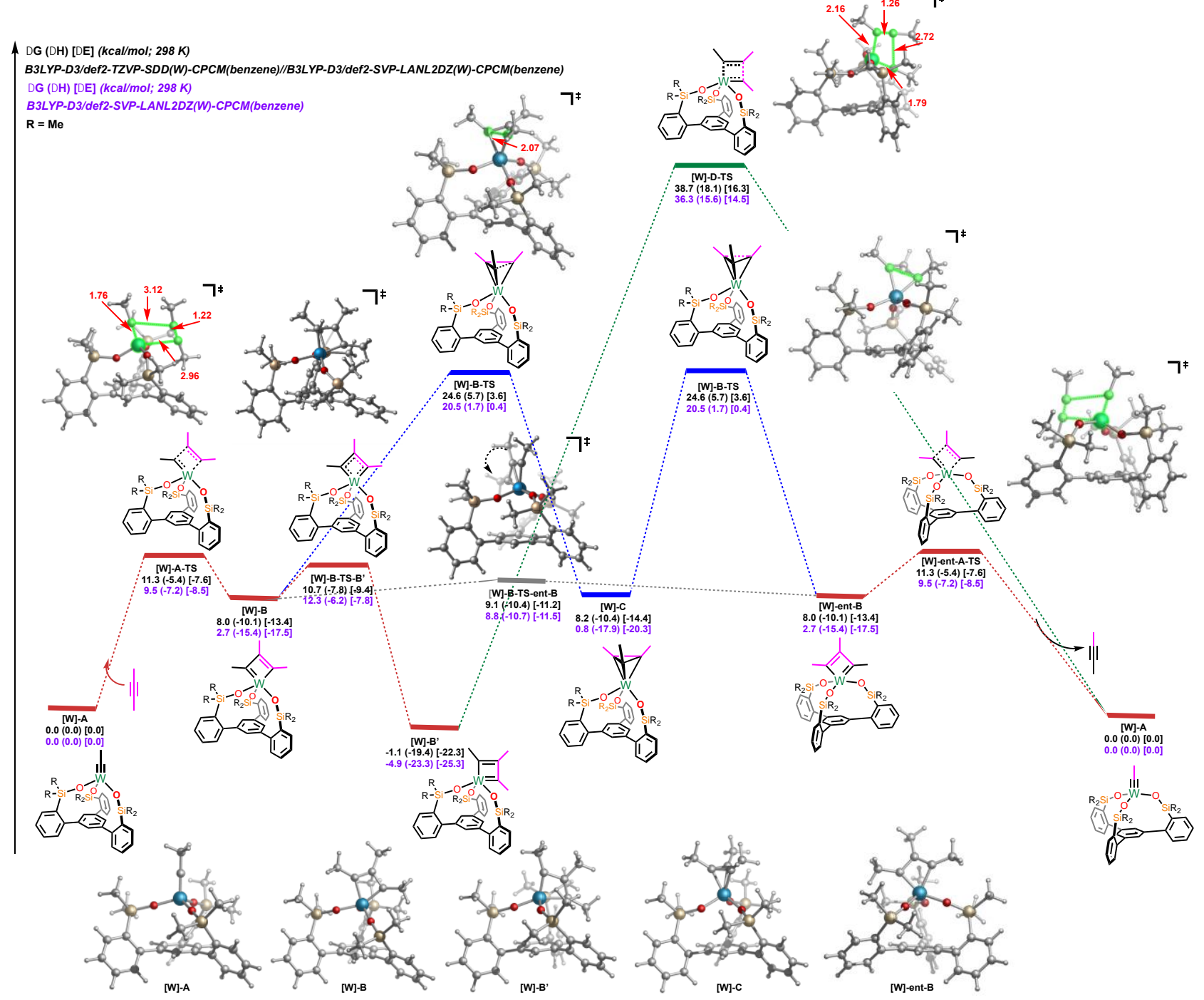

Figure S60. Energetics of MCBD and MTd formation via [2+2] cycloaddition for tungsten with $\mathrm{SiP}^{\mathrm{Me}}$ system computed at the B3LYP-D3/def2TZVP-SDD(W)-CPCM(benzene)// B3LYP-D3/def2SVP-LANL2DZ(W)-CPCM(benzene)level of theory (black) and the PBEPBE/def2TZVP-SDD(W)-CPCM(benzene)// B3LYP-D3/def2SVP-LANL2DZ(W)-CPCM(benzene)level of theory (purple). 


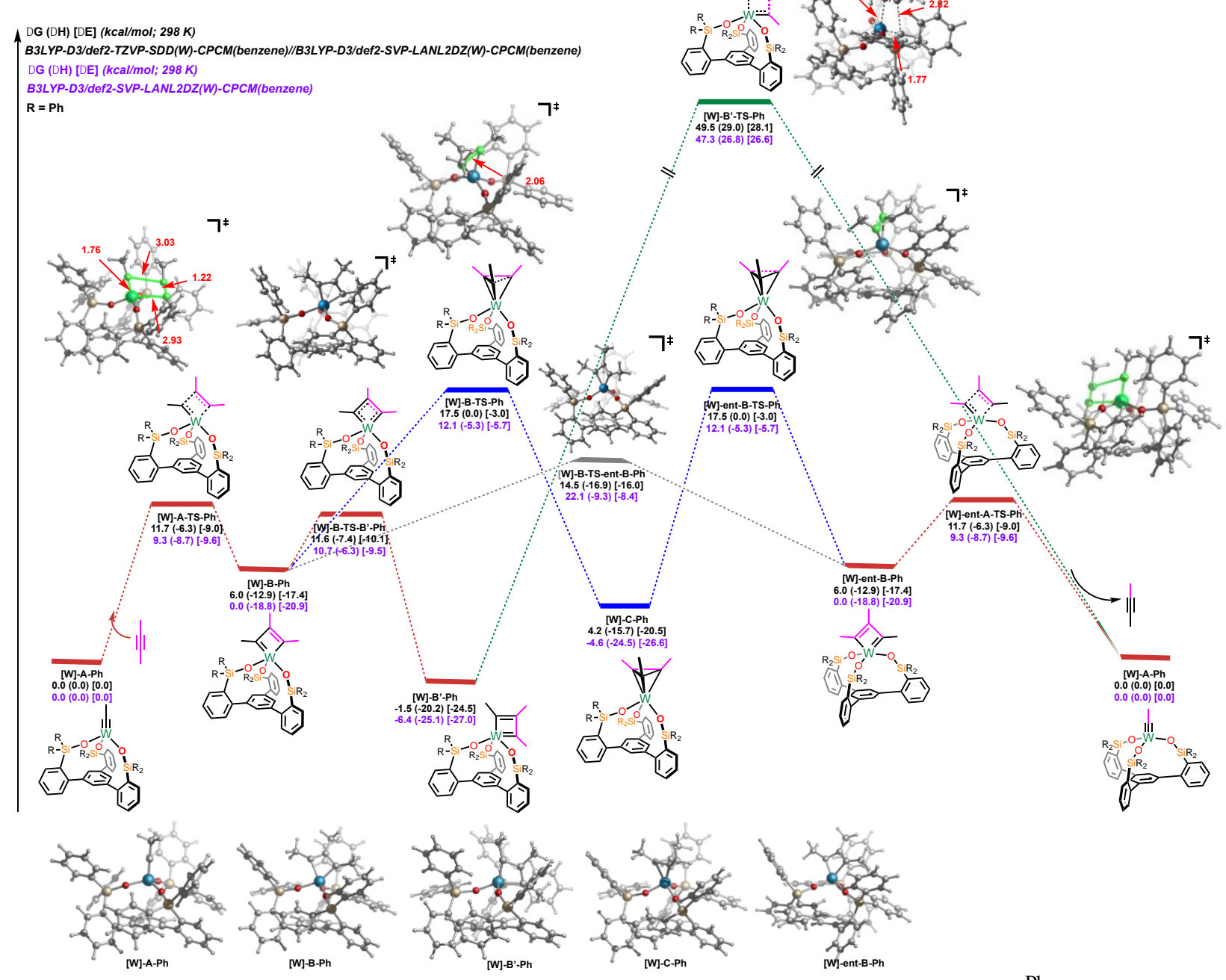

Figure S61. Energetics of MCBD and MTd formation via [2+2] cycloaddition for tungsten with $\mathrm{SiP}^{\mathrm{Ph}}$ system computed at the B3LYP-D3/def2TZVP-SDD(W)-CPCM(benzene)// B3LYP-D3/def2SVP-LANL2DZ(W)-CPCM(benzene)level of theory (outside parenthesis) and the PBEPBE/def2TZVP-SDD(W)-CPCM(benzene)// B3LYP-D3/def2SVP-LANL2DZ(W)-CPCM(benzene)level of theory (inside parenthesis). 

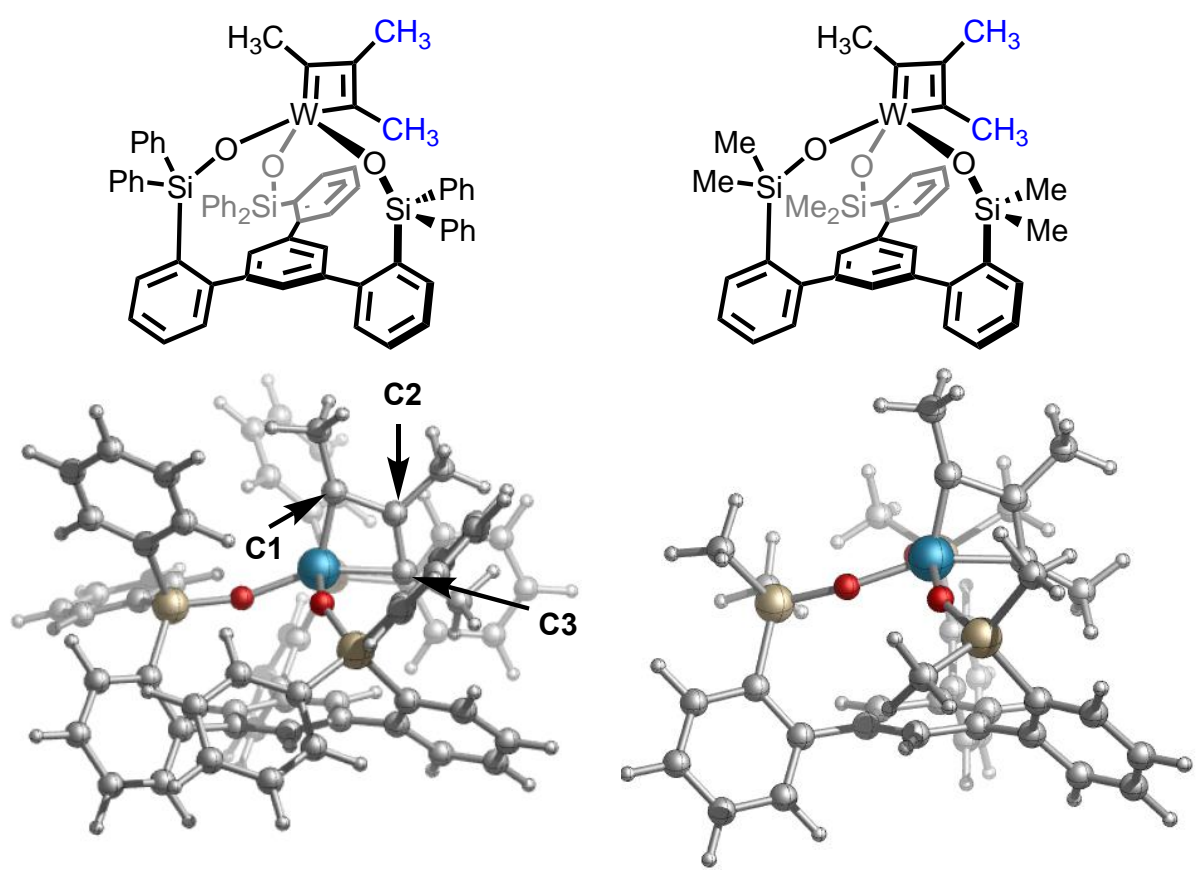

\begin{tabular}{lcccc} 
& $\begin{array}{c}\text { [W]-B'-Ph } \\
\text {-1.5 }\end{array}$ & & \multicolumn{2}{c}{$\begin{array}{c}\text { [W]-B'-Me } \\
-1.1\end{array}$} \\
& {$[$ [W]-B'-Ph } & MCBD4 & {$[$ [W]-B'-Me } & MCBD5 \\
\hline W1-C1 & 1.868 & 1.862 & 1.862 & 1.865 \\
C1-C2 & 1.511 & 1.493 & 1.525 & 1.487 \\
C2-C3 & 1.401 & 1.412 & 1.387 & 1.415 \\
C3-W1 & 1.994 & 1.982 & 2.010 & 1.967 \\
W1-C1-C2 & 79.74 & 78.24 & 80.11 & 78.89 \\
C1-C2-C3 & 120.58 & 122.3 & 120.37 & 121.41 \\
C2-C3-W1 & 77.88 & 76.11 & 78.12 & 77.04 \\
C3-W1-C1 & 81.80 & 83.28 & 81.40 & 82.63
\end{tabular}

Table S7. List of Bond Lengths $(\AA)$ and Angles $\left({ }^{\circ}\right)$ for computed metallacyclobutadienes [W]B'-Ph and [W]-B'-Me compared to the analogous isolated structures MCBD4 and MCBD5. 


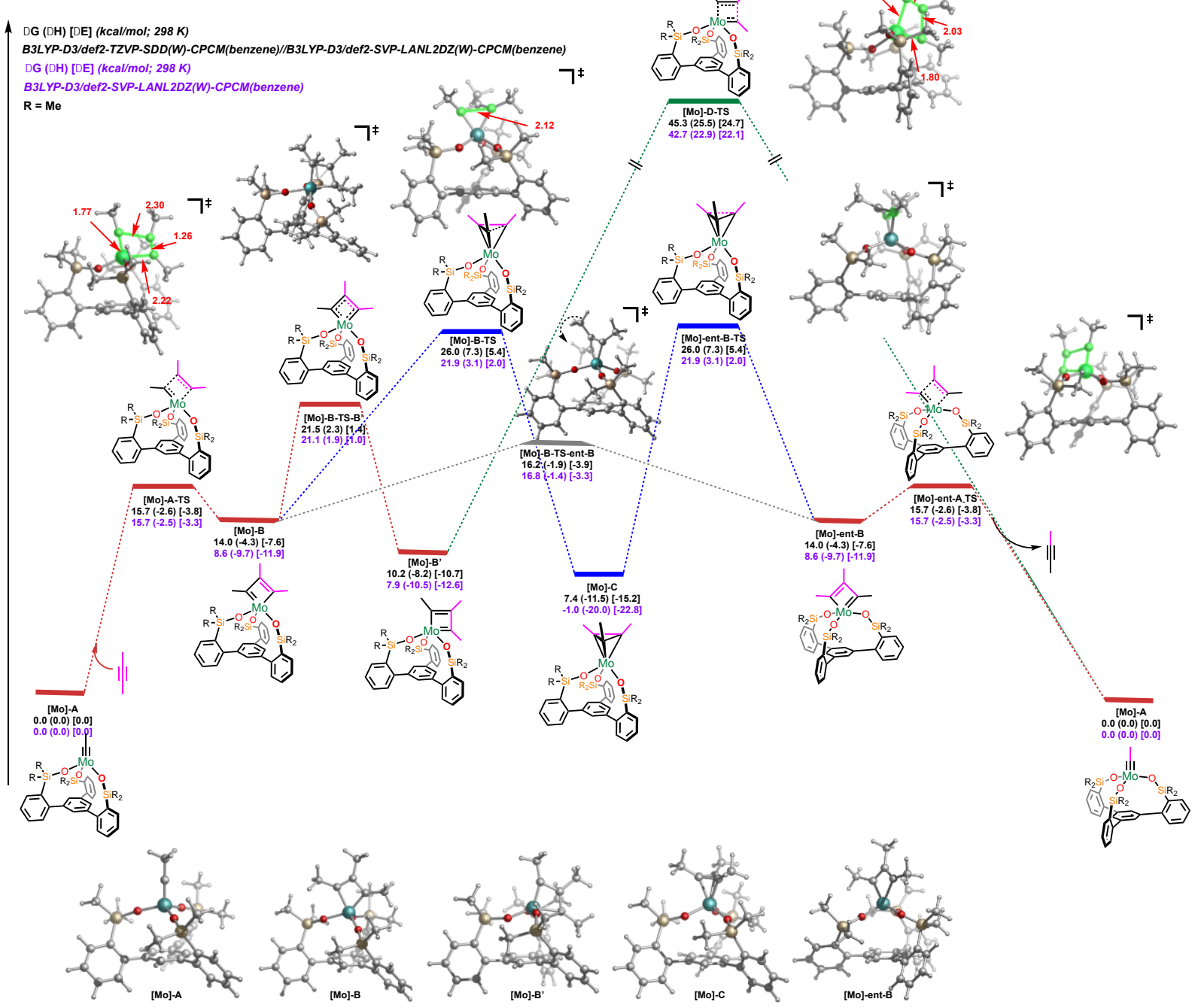

Figure S62. Energetics of MCBD and MTd formation via [2+2] cycloaddition for molybdenum with $\mathrm{SiP}^{\mathrm{Me}}$ system computed at the B3LYP-D3/def2TZVP-SDD(Mo)-CPCM(benzene)// B3LYP-D3/def2SVP-LANL2DZ(Mo)-CPCM(benzene)level of theory (outside parenthesis) and the PBEPBE/def2TZVP-SDD(Mo)-CPCM(benzene)// B3LYP-D3/def2SVP-LANL2DZ(Mo)-CPCM(benzene)level of theory (inside parenthesis). 


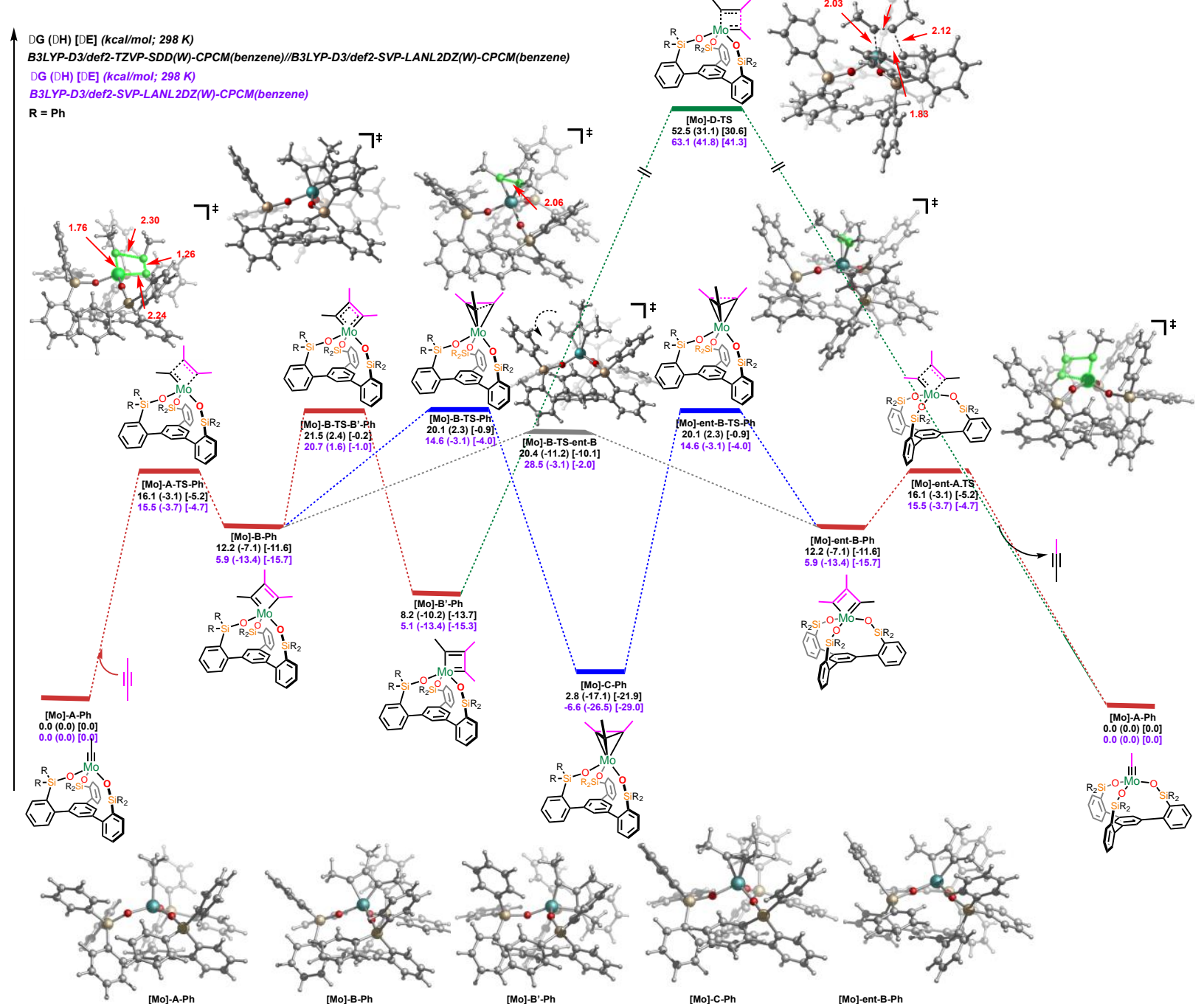

Figure S63. Energetics of MCBD and MTd formation via [2+2] cycloaddition for molybdenum with $\mathrm{SiP}^{\mathrm{Ph}}$ system computed at the B3LYP-D3/def2TZVP-SDD(Mo)-CPCM(benzene)// B3LYP-D3/def2SVP-LANL2DZ(Mo)-CPCM(benzene)level of theory (outside parenthesis) and the PBEPBE/def2TZVP-SDD(Mo)-CPCM(benzene)// B3LYP-D3/def2SVP-LANL2DZ(Mo)-CPCM(benzene)level of theory (inside parenthesis). 


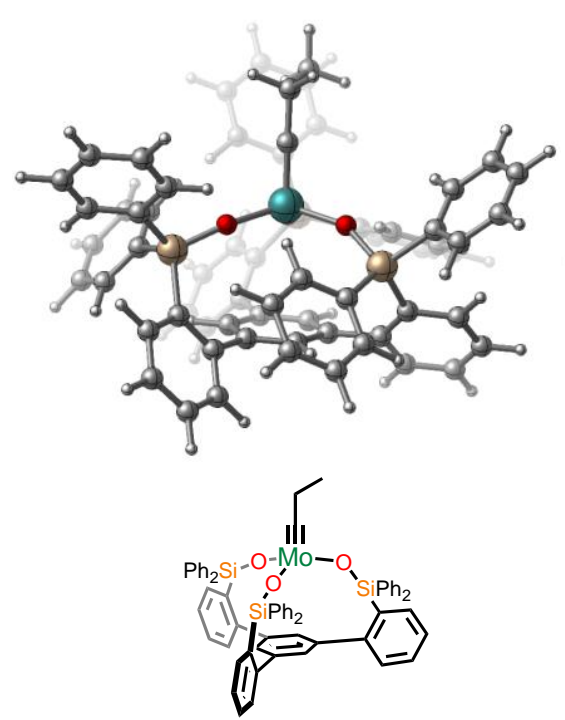

0.0

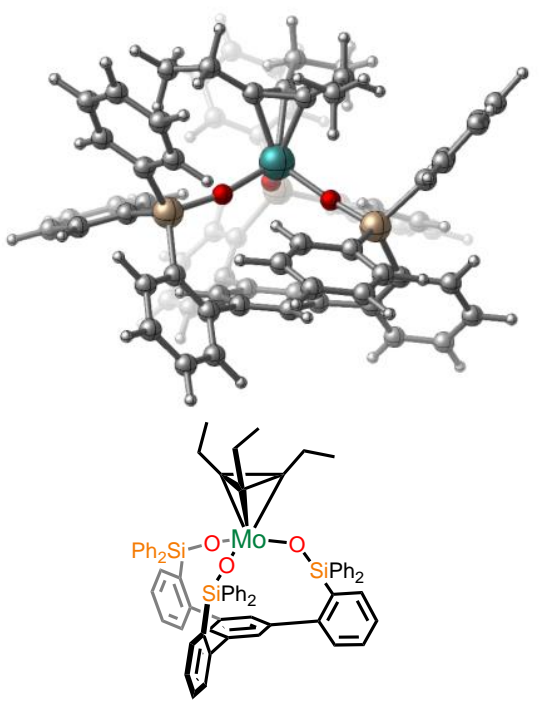

$-2.7$

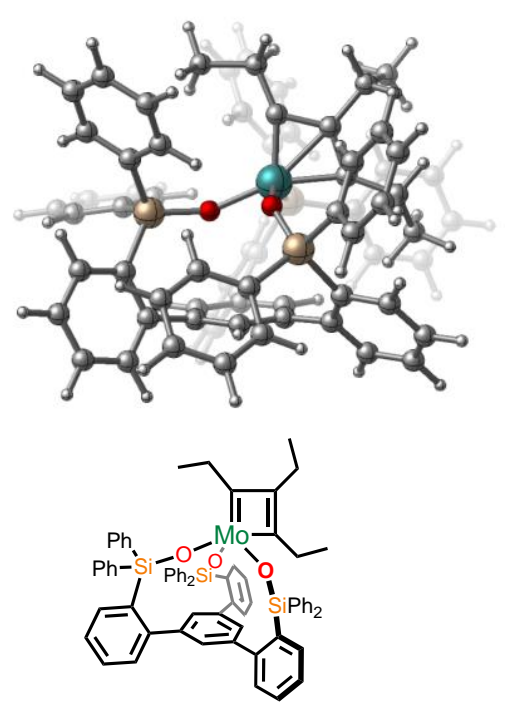

11.4

Figure S64. Energetics of MCBD and $\mathrm{MT}_{\mathrm{d}}$ formation for molybdenum with $\mathbf{S i P}^{\mathbf{P h}}$ ligand and 3-hexyne substrate. Free energies $(\mathrm{kcal} / \mathrm{mol})$ are computed at the B3LYP-D3/def2TZVP-SDD(Mo)-CPCM(benzene)// B3LYP-D3/def2SVP-LANL2DZ(Mo)CPCM(benzene)level of theory. 


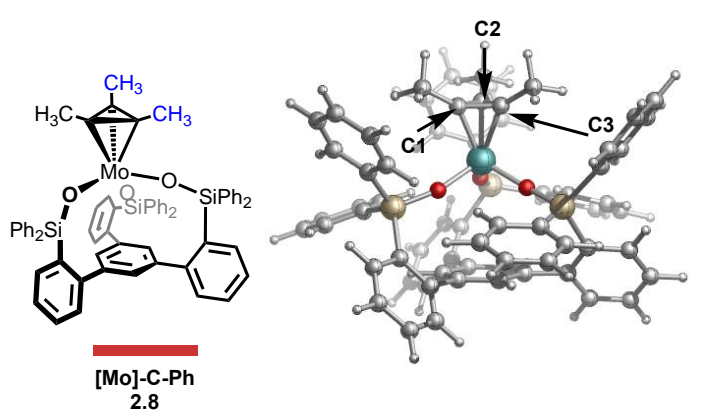

$\begin{array}{lcc} & \text { [Mo]-C-Ph } & \text { MTd1 } \\ \text { Mo-C1 } & 2.093 & 2.064 \\ \text { Mo-C2 } & 2.094 & 2.065 \\ \text { Mo-C3 } & 2.094 & 2.064 \\ \text { C1-C2 } & 1.453 & 1.445 \\ \text { C2-C3 } & 1.454 & 1.458 \\ \text { C3-C1 } & 1.451 & 1.458 \\ \text { Mo-C1-C2 } & 69.65 & 69.2 \\ \text { C1-C2-C3 } & 59.89 & 60.3 \\ \text { C2-C3-Mo } & 69.64 & 69.0 \\ \text { C3-Mo-C1 } & 40.54 & 41.4\end{array}$

Table S8. List of Bond Lengths $(\AA)$ and Angles $\left(^{\circ}\right)$ for computed metallatetrahedrane [Mo]-CPh compared to the analogous isolated structure MTd1. 

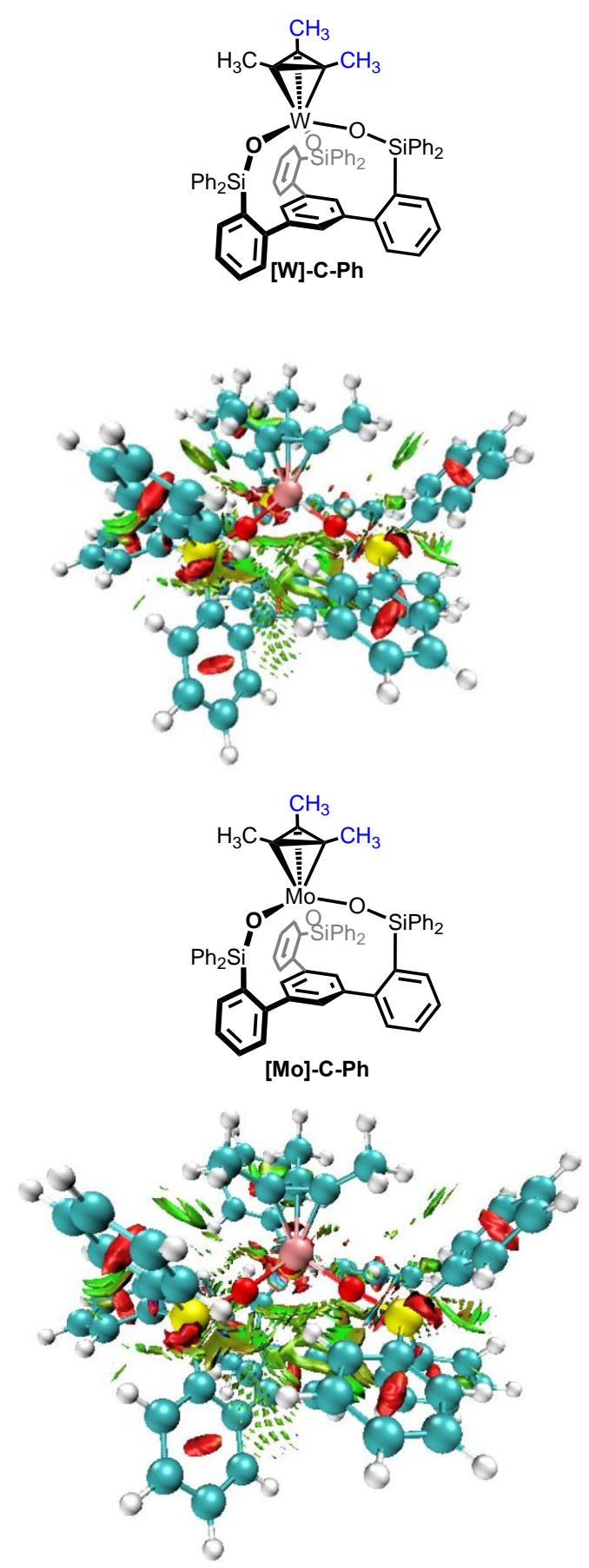
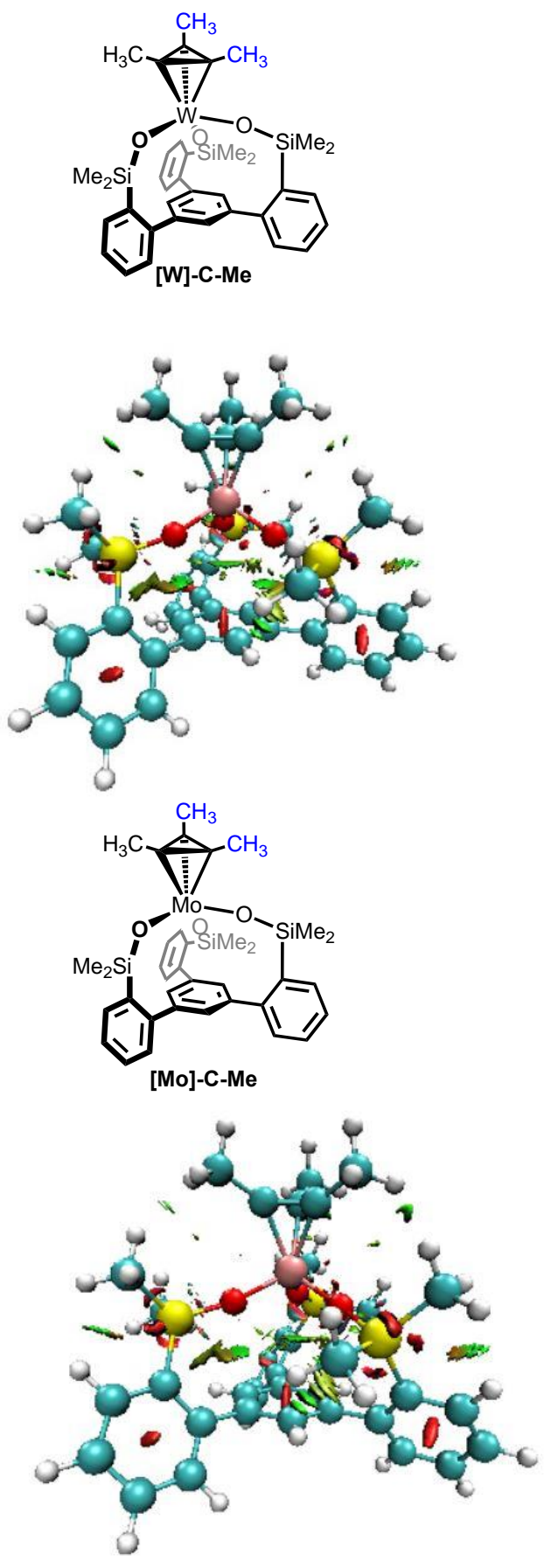

Figure S65. NCI plots showing the comparison between metallatetrahedranes in the tungsten (top) and molybdenum (bottom) systems with both the $\mathrm{SiP}^{\mathrm{Ph}}$ and $\mathrm{SiP}^{\mathrm{Me}}$ ligands. Key C-H $\cdots \pi$ noncovalent stabilizing interactions are visible in the NCI plots of the $\mathrm{SiP}^{\mathrm{Ph}}$ metallatetrahedranes, as indicated by the green colors between substrate $\mathrm{C}-\mathrm{H}$ and ligand aryl groups. 

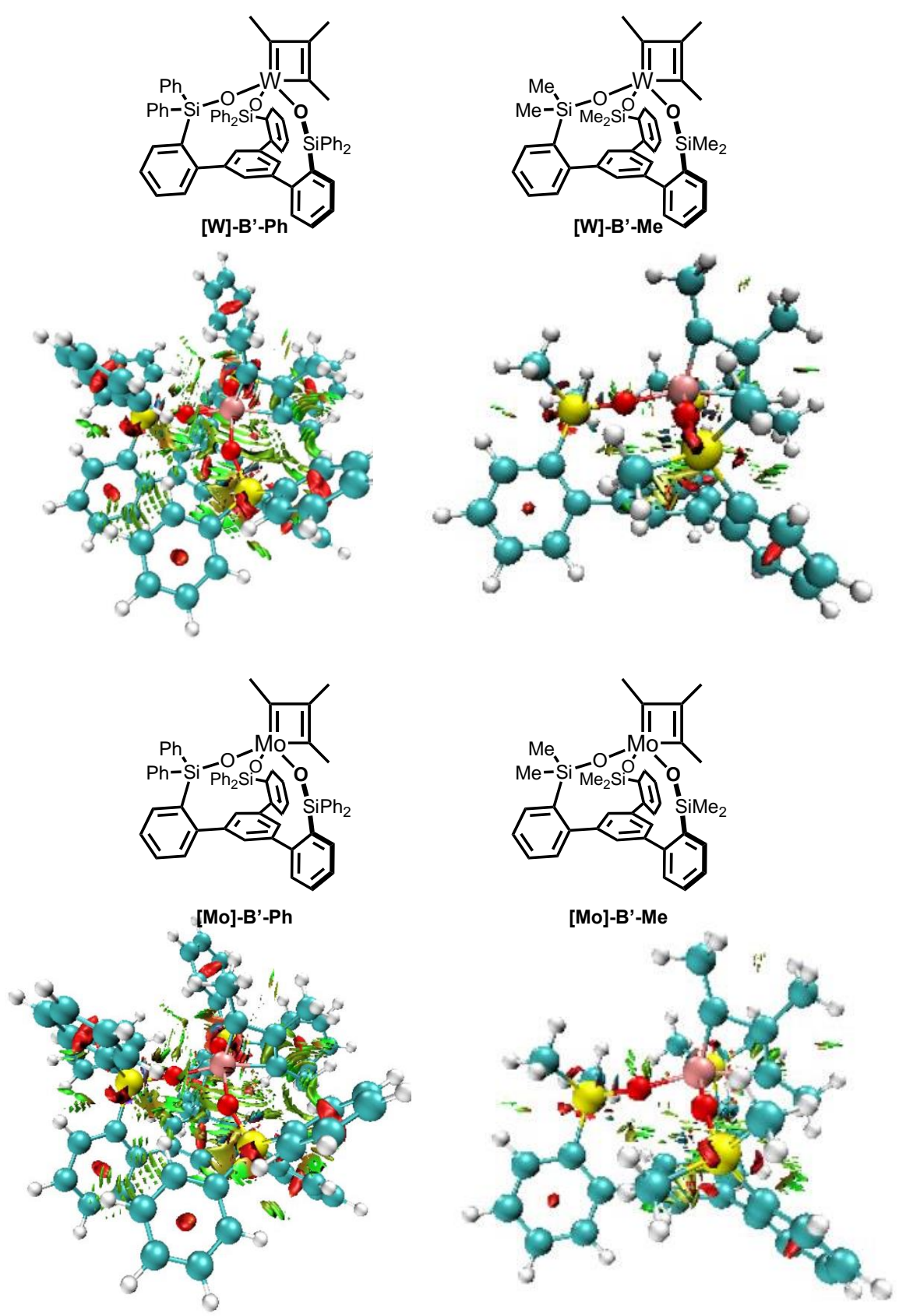

Figure S66. NCI plots showing the comparison between metallacyclobutadienes in the tungsten (top) and molybdenum (bottom) systems with both the $\mathrm{SiP}^{\mathrm{Ph}}$ and $\mathrm{SiP}^{\mathrm{Me}}$ ligands. Key $\mathrm{C}-\mathrm{H}^{\cdots} \pi$ noncovalent stabilizing interactions visible in the NCI plots of the $\mathrm{SiP}^{\mathrm{Ph}}$ metallatetrahedranes are no longer present in the MCBD structures. 


\section{Starting Structure}

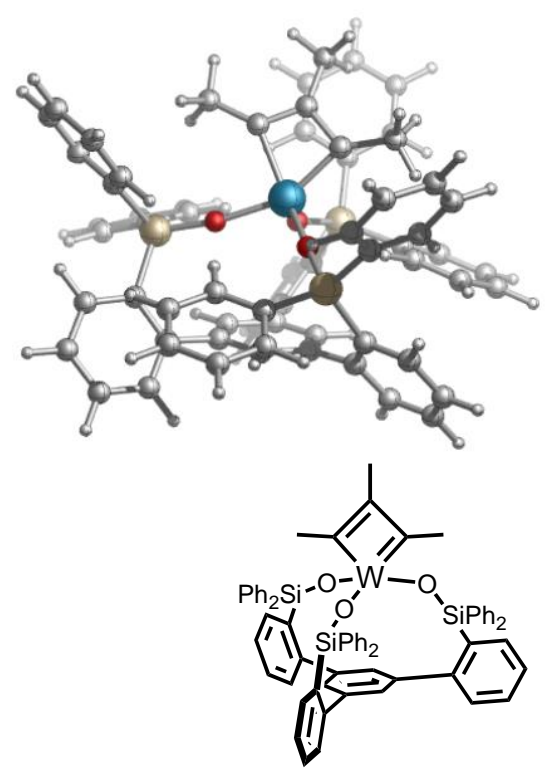

$\Delta \mathrm{E}_{\text {elstat }}=-2.0 \mathrm{kcal} / \mathrm{mol}$

Starting Structure
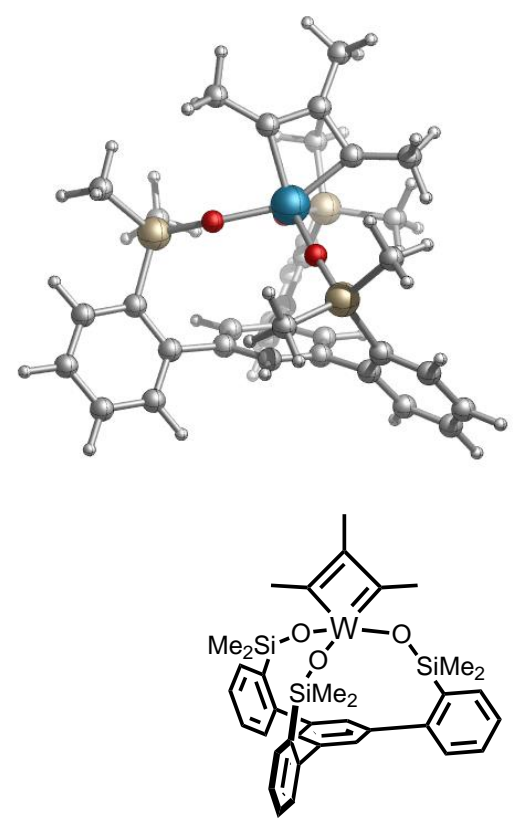

$\Delta \mathrm{E}_{\text {elstat }}=-6.2 \mathrm{kcal} / \mathrm{mol}$
Decomposed Structure

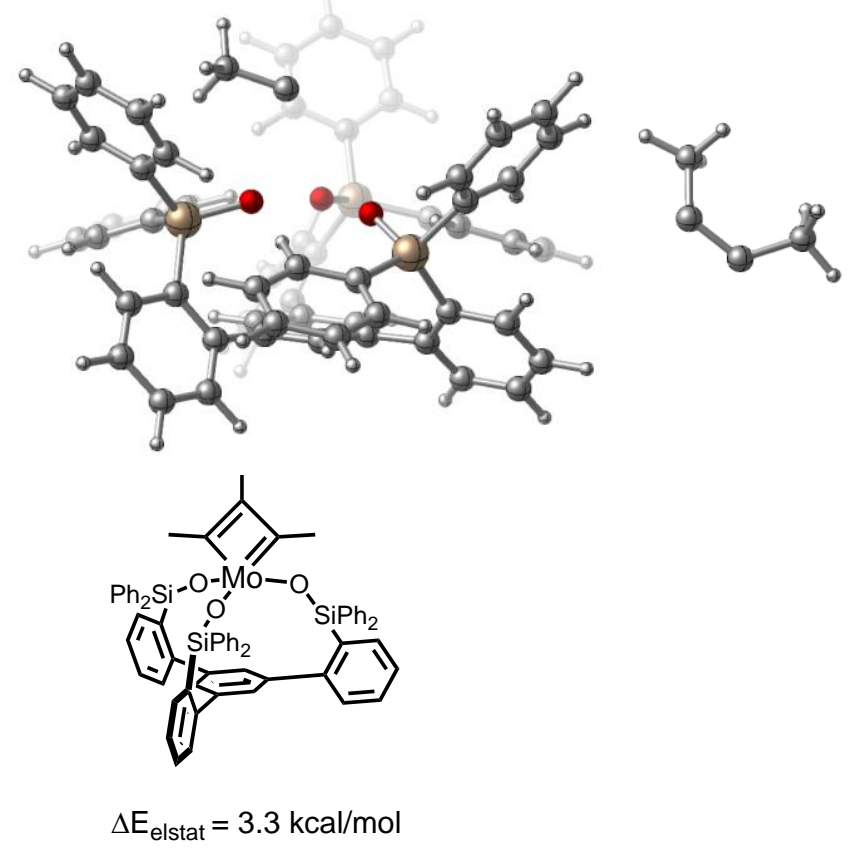

Decomposed Structure
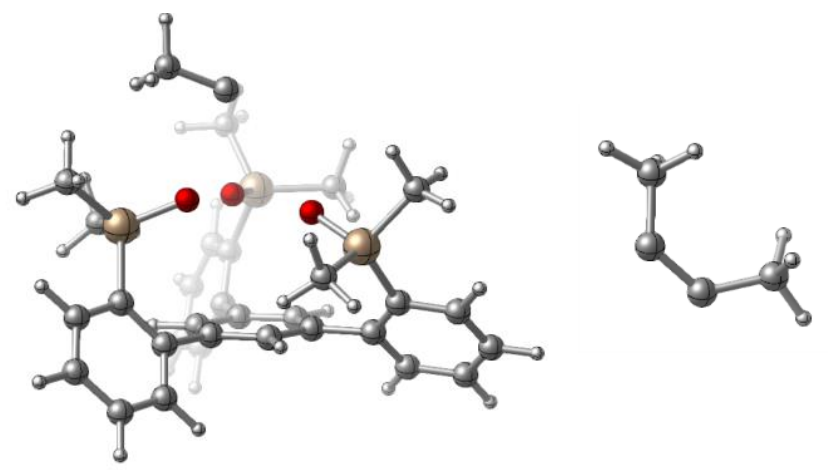

$\Delta \mathrm{E}_{\text {elstat }}=-0.9 \mathrm{kcal} / \mathrm{mol}$

Figure S67. Electrostatic energies for the MCBD intermediates, [W]-B and [Mo]-B, computed by the second-generation ALMO-EDA method using HF/6-311G(d,p) with the shown decomposed intermediates from the given starting structure. The electrostatic energies are lower for [W]-B than [Mo]-B and thus the electrostatic energy likely contributes to tungsten's preference to form the MCBD. 

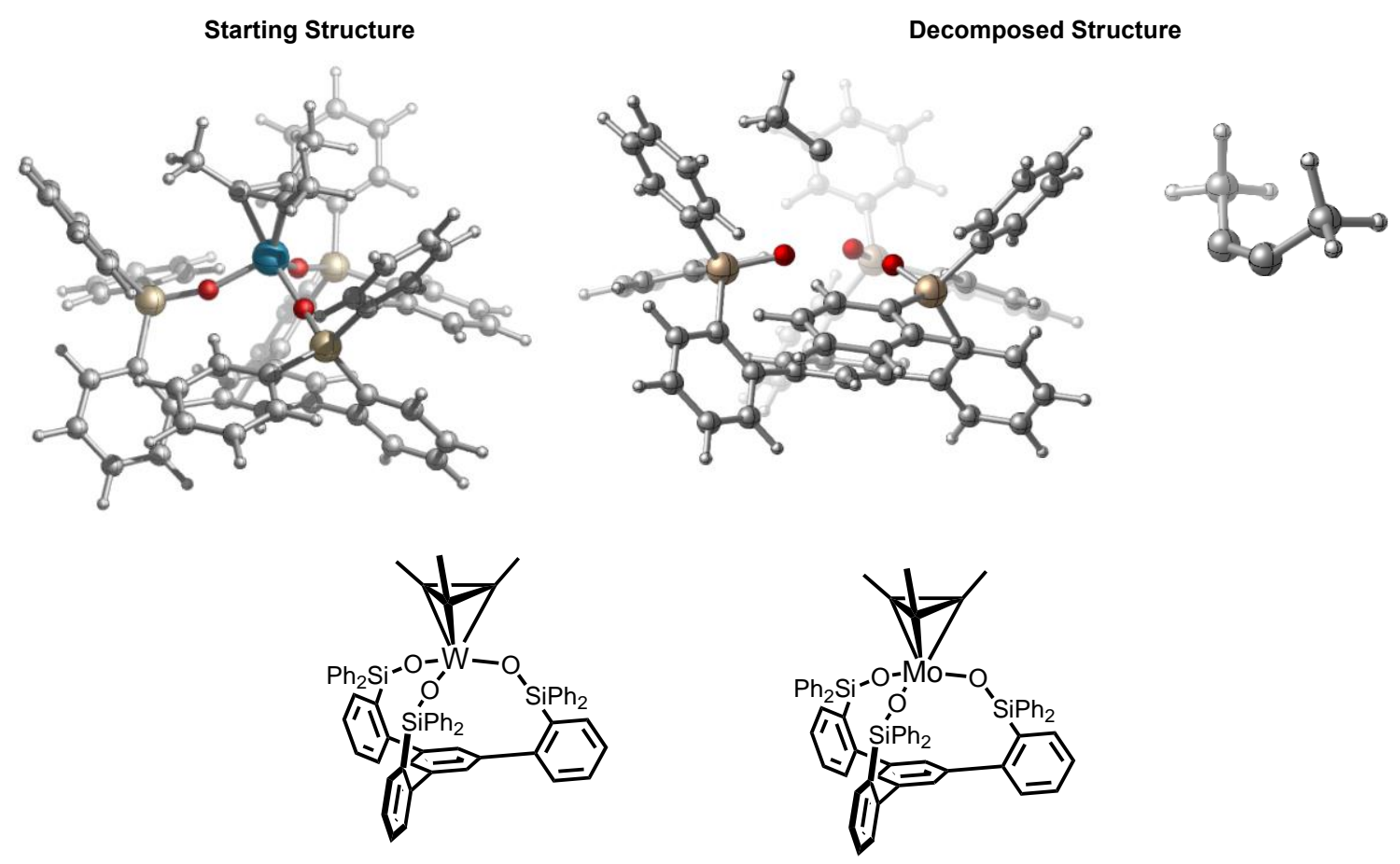

$\Delta \mathrm{E}_{\text {elstat }}=-29.2 \mathrm{kcal} / \mathrm{mol}$

$\Delta \mathrm{E}_{\text {elstat }}=-30.5 \mathrm{kcal} / \mathrm{mol}$
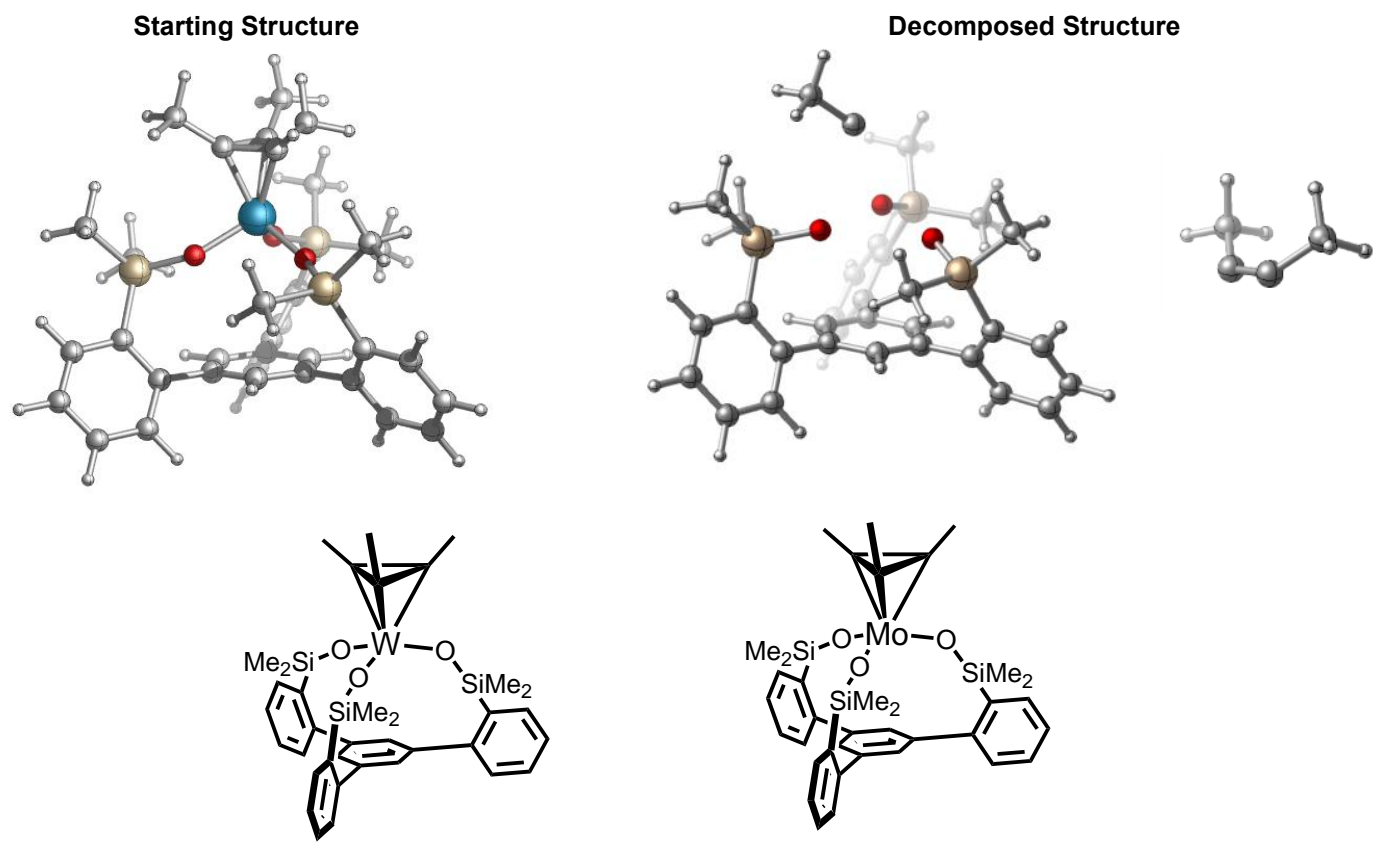

$\Delta \mathrm{E}_{\text {elstat }}=-24.9 \mathrm{kcal} / \mathrm{mol}$

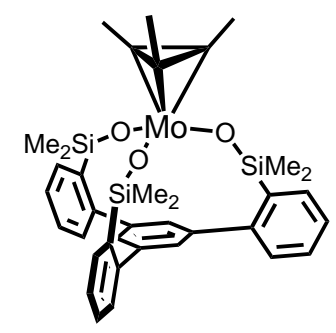

$\Delta \mathrm{E}_{\text {elstat }}=-29.4 \mathrm{kcal} / \mathrm{mol}$

Figure S68. Electrostatic energies for the MTd intermediates, [W]-C and [Mo]-C, computed by the second-generation ALMO-EDA method using HF/6-311G(d,p) with the shown decomposed intermediates from the given starting structure. The electrostatic energies are lower for [Mo]-C than [W]-C and thus the electrostatic energy likely contributes to molybdenum's preference to form the MTd. 

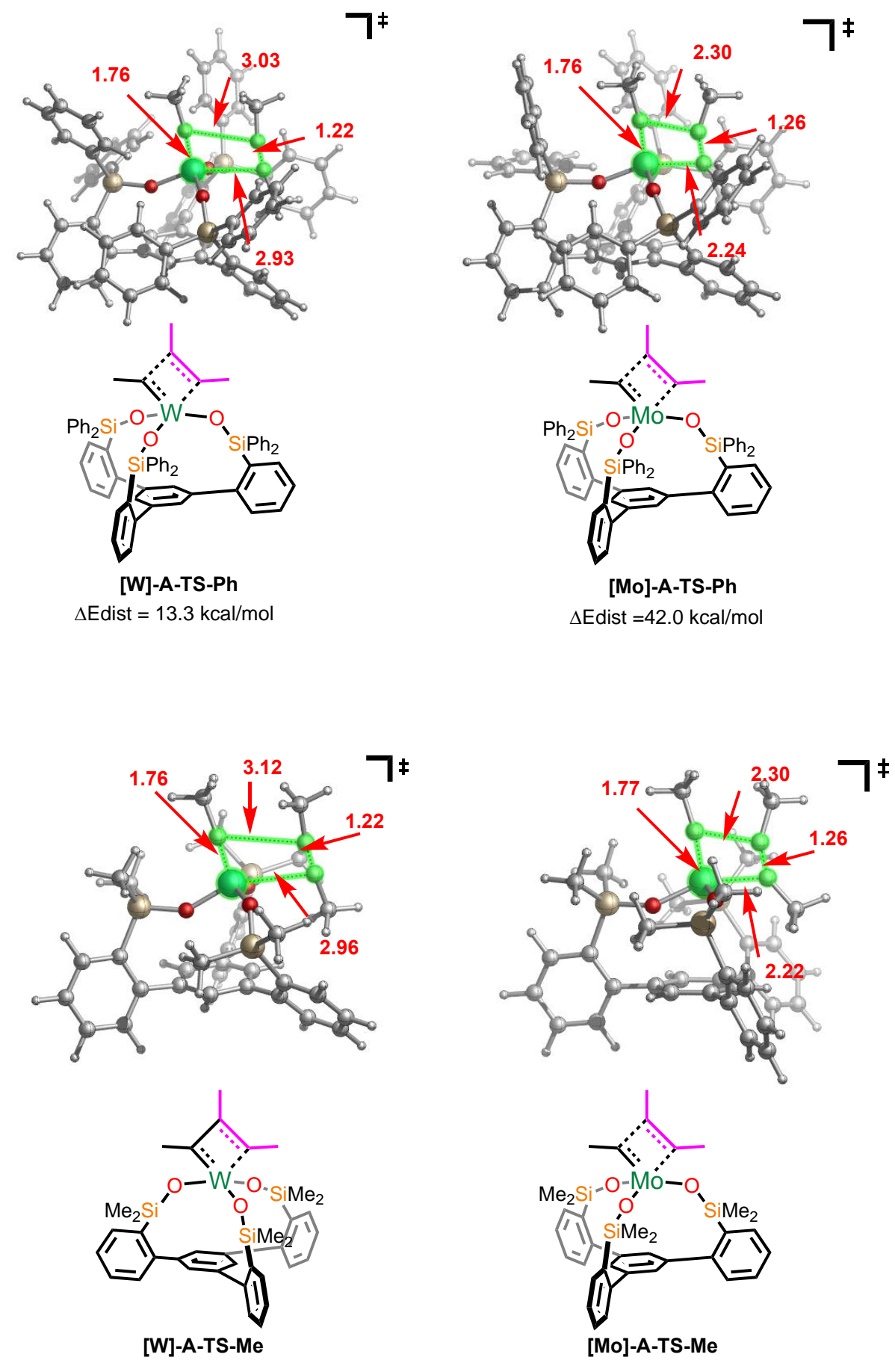

Figure S69. Distortion energies for the formation of the MCBD intermediates, [W]-B and [Mo]B, computed at the B3LYP-D3/def2TZVP-SDD(Mo)-CPCM(benzene)// B3LYP-D3/def2SVPLANL2DZ(Mo)-CPCM(benzene) level of theory. The low distortion energy required to form [W]-B compared to that required to form [Mo]-B provides insight into the preference for the MCBD in the case of tungsten. 

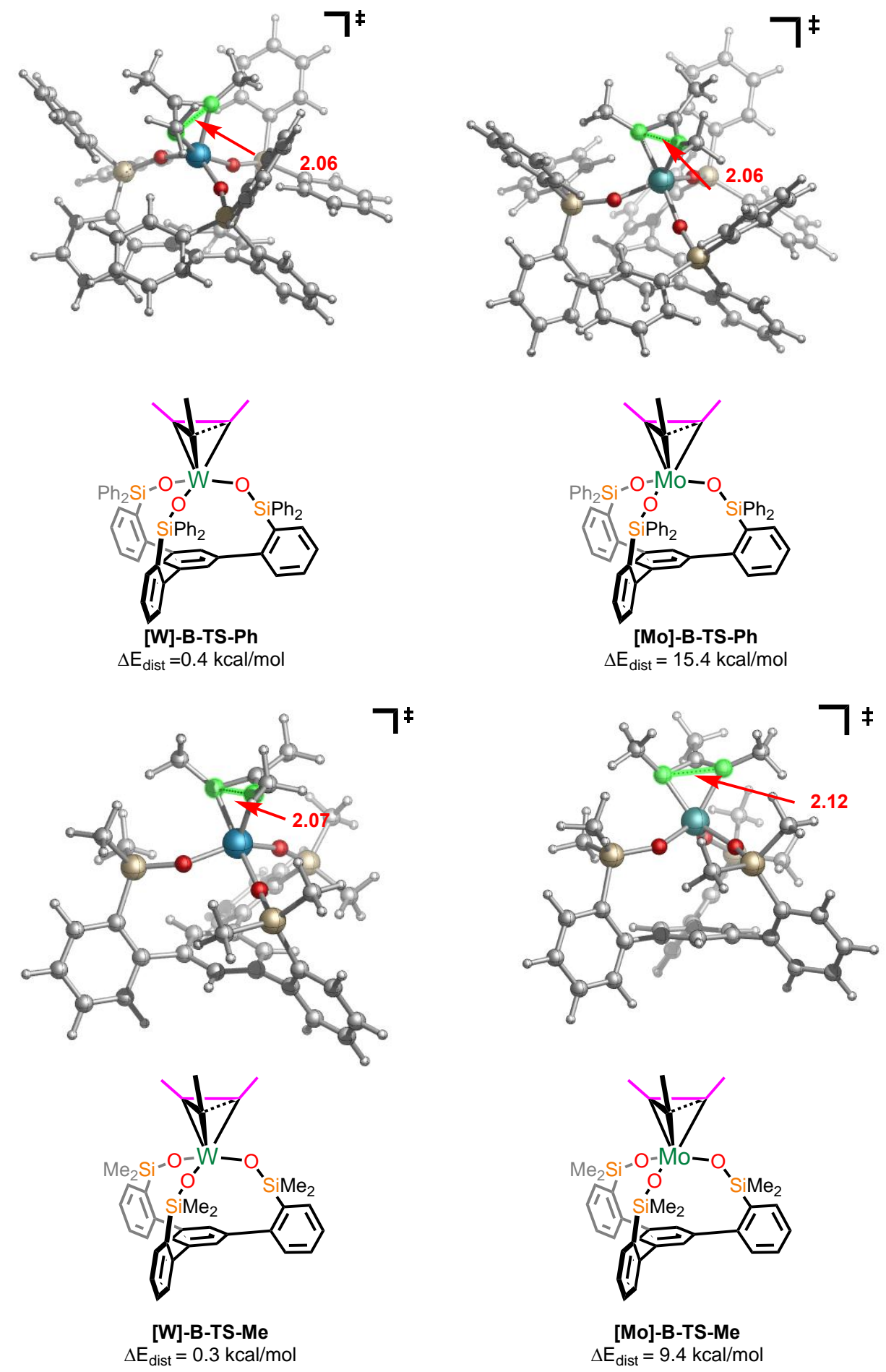

Figure S70. Distortion energies for the formation of the MTd intermediates, [W]-C and [Mo]-C, computed at the B3LYP-D3/def2TZVP-SDD(Mo)-CPCM(benzene)// B3LYP-D3/def2SVPLANL2DZ(Mo)-CPCM(benzene) level of theory. In this case, the distortion energy to form [W]$\mathrm{C}$ is lower than that to form [Mo]-C, and therefore the distortion energy does not control the preferred formation of the MTd intermediate in the molybdenum case. 


\section{Calculated Structures and Energies}

[W]-A

B3LYP-D3/def2SVP-LANL2DZ(W)-CPCM(benzene)

Zero-point correction $=0.588432($ Hartree/Particle $)$

Thermal correction to Energy= 0.631637

Thermal correction to Enthalpy $=0.632582$

Thermal correction to Gibbs Free Energy $=0.511741$

Sum of electronic and zero-point Energies $=-2401.637346$

Sum of electronic and thermal Energies $=-2401.594140$

Sum of electronic and thermal Enthalpies= -2401.593196

Sum of electronic and thermal Free Energies $=-2401.714037$

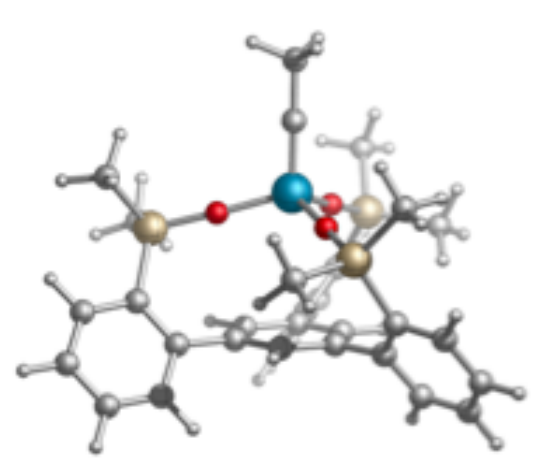

B3LYP-D3/def2TZVP-SDD(W)-CPCM(benzene)//B3LYP-D3/def2SVP-LANL2DZ(W)CPCM(benzene)

$\mathrm{HF}=-2403.4449347$

PBEPBE/def2TZVP-SDD(W)-CPCM(benzene)//B3LYP-D3/def2SVP-LANL2DZ(W)$\mathrm{CPCM}($ benzene)

$\mathrm{HF}=-2400.9668649$

\section{[W]-A-TS}

\section{B3LYP-D3/def2SVP-LANL2DZ(W)-CPCM(benzene)}

Imaginary frequency $=-64.00 \mathrm{~cm}^{-1}$

Zero-point correction $=0.675502($ Hartree/Particle $)$

Thermal correction to Energy $=0.723675$

Thermal correction to Enthalpy $=0.724619$

Thermal correction to Gibbs Free Energy= 0.595066

Sum of electronic and zero-point Energies $=-2557.434867$

Sum of electronic and thermal Energies $=-2557.386694$

Sum of electronic and thermal Enthalpies $=-2557.385750$

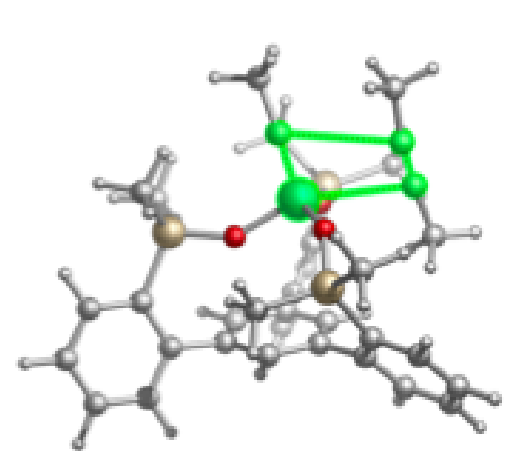

Sum of electronic and thermal Free Energies $=-2557.515303$

B3LYP-D3/def2TZVP-SDD(W)-CPCM(benzene)//B3LYP-D3/def2SVP-LANL2DZ(W)$\mathrm{CPCM}($ benzene $)$

$\mathrm{HF}=-2559.5021997$

PBEPBE/def2TZVP-SDD(W)-CPCM(benzene)//B3LYP-D3/def2SVP-LANL2DZ(W)CPCM(benzene)

$\mathrm{HF}=-2556.7746835$ 
[W]-B

B3LYP-D3/def2SVP-LANL2DZ(W)-CPCM(benzene)

Zero-point correction $=0.677424$ (Hartree/Particle)

Thermal correction to Energy $=0.725025$

Thermal correction to Enthalpy $=0.725969$

Thermal correction to Gibbs Free Energy $=0.598515$

Sum of electronic and zero-point Energies $=-2557.447293$

Sum of electronic and thermal Energies $=-2557.399692$

Sum of electronic and thermal Enthalpies $=-2557.398748$

Sum of electronic and thermal Free Energies $=-2557.526203$

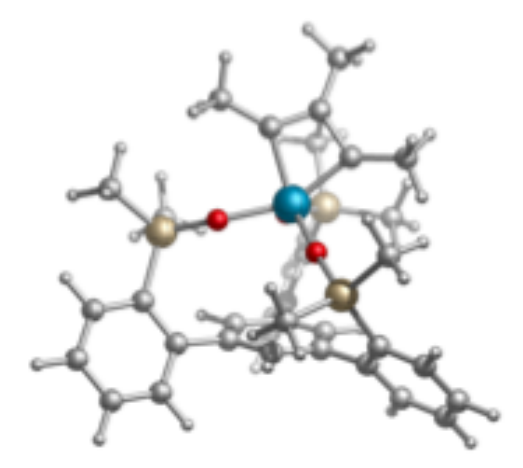

B3LYP-D3/def2TZVP-SDD(W)-CPCM(benzene)//B3LYP-D3/def2SVP-LANL2DZ(W)-

CPCM(benzene)

$\mathrm{HF}=-2559.5114664$

PBEPBE/def2TZVP-SDD(W)-CPCM(benzene)//B3LYP-D3/def2SVP-LANL2DZ(W)CPCM(benzene)

$\mathrm{HF}=-2556.80022$

\section{[W]-B-TS-B'}

B3LYP-D3/def2SVP-LANL2DZ(W)-CPCM(benzene)

Imaginary frequency $=-135.61 \mathrm{~cm}^{-1}$

Zero-point correction $=$

Thermal correction to Energy=

0.676479 (Hartree/Particle)

Thermal correction to Enthalpy= $\quad 0.724523$

0.723579

Thermal correction to Gibbs Free Energy= 0.597659

Sum of electronic and zero-point Energies $=\quad-2557.441541$

Sum of electronic and thermal Energies= $\quad-2557.394440$

Sum of electronic and thermal Enthalpies $=\quad-2557.393496$

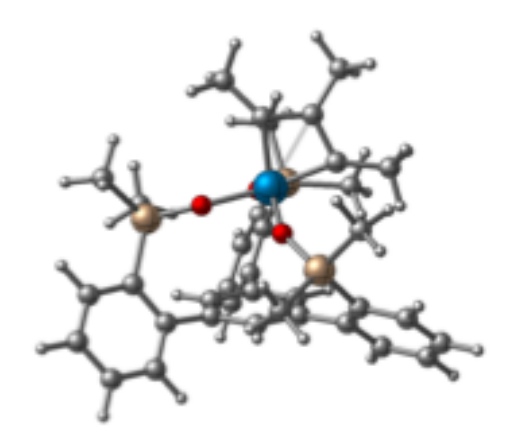

Sum of electronic and thermal Free Energies $=\quad-2557.520361$

B3LYP-D3/def2TZVP-SDD(W)-CPCM(benzene)//B3LYP-D3/def2SVP-LANL2DZ(W)-

CPCM(benzene)

$\mathrm{HF}=-2559.5050943$

PBEPBE/def2TZVP-SDD(W)-CPCM(benzene)//B3LYP-D3/def2SVP-LANL2DZ(W)-

CPCM(benzene)

$\mathrm{HF}=-2556.7945369$ 
[W]-B'

B3LYP-D3/def2SVP-LANL2DZ(W)-CPCM(benzene)

Zero-point correction $=0.677501$ (Hartree/Particle)

Thermal correction to Energy $=0.725005$

Thermal correction to Enthalpy $=0.725949$

Thermal correction to Gibbs Free Energy $=0.598897$

Sum of electronic and zero-point Energies $=-2557.459719$

Sum of electronic and thermal Energies $=-2557.412216$

Sum of electronic and thermal Enthalpies $=-2557.411271$

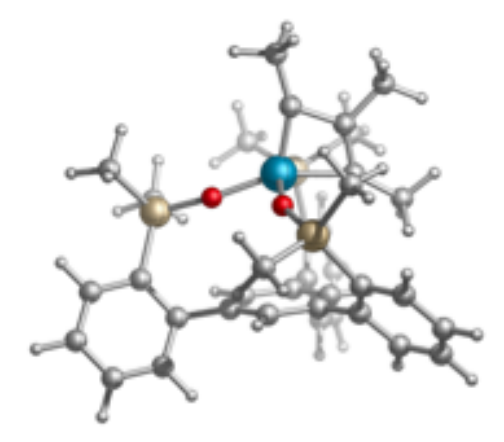

Sum of electronic and thermal Free Energies $=-2557.538324$

B3LYP-D3/def2TZVP-SDD(W)-CPCM(benzene)//B3LYP-D3/def2SVP-LANL2DZ(W)-

CPCM(benzene)

$\mathrm{HF}=-2559.5256023$

PBEPBE/def2TZVP-SDD(W)-CPCM(benzene)//B3LYP-D3/def2SVP-LANL2DZ(W)CPCM(benzene)

$\mathrm{HF}=-2556.8148769$

\section{[W]-B-TS-ent-B}

B3LYP-D3/def2SVP-LANL2DZ(W)-CPCM(benzene)

Imaginary frequency $=-35.17 \mathrm{~cm}^{-1}$

Zero-point correction=

0.676893 (Hartree/Particle)

Thermal correction to Energy=

Thermal correction to Enthalpy=

0.723559

Thermal correction to Gibbs Free Energy $=\quad 0.599376$

Sum of electronic and zero-point Energies $=\quad-2557.443042$

Sum of electronic and thermal Energies= $\quad-2557.396376$

Sum of electronic and thermal Enthalpies $=\quad-2557.395432$

Sum of electronic and thermal Free Energies $=\quad-2557.520560$

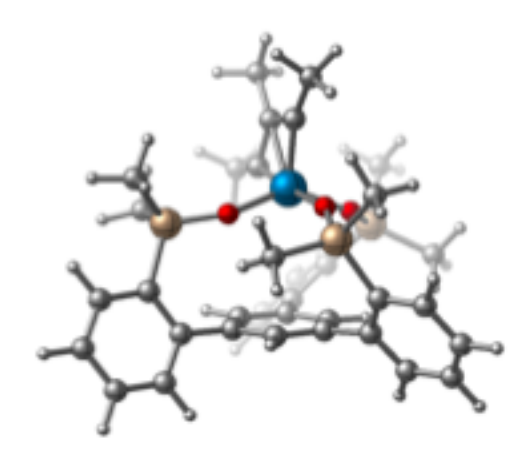

B3LYP-D3/def2TZVP-SDD(W)-CPCM(benzene)//B3LYP-D3/def2SVP-LANL2DZ(W)-

CPCM(benzene)

$\mathrm{HF}=-2559.5078935$

PBEPBE/def2TZVP-SDD(W)-CPCM(benzene)//B3LYP-D3/def2SVP-LANL2DZ(W)-

CPCM(benzene)

$\mathrm{HF}=-2556.8004277$ 
[W]-C

B3LYP-D3/def2SVP-LANL2DZ(W)-CPCM(benzene)

Zero-point correction $=0.678449$ (Hartree/Particle)

Thermal correction to Energy $=0.725649$

Thermal correction to Enthalpy $=0.726593$

Thermal correction to Gibbs Free Energy= 0.600119

Sum of electronic and zero-point Energies $=-2557.450847$

Sum of electronic and thermal Energies $=-2557.403648$

Sum of electronic and thermal Enthalpies $=-2557.402703$

Sum of electronic and thermal Free Energies $=-2557.529177$

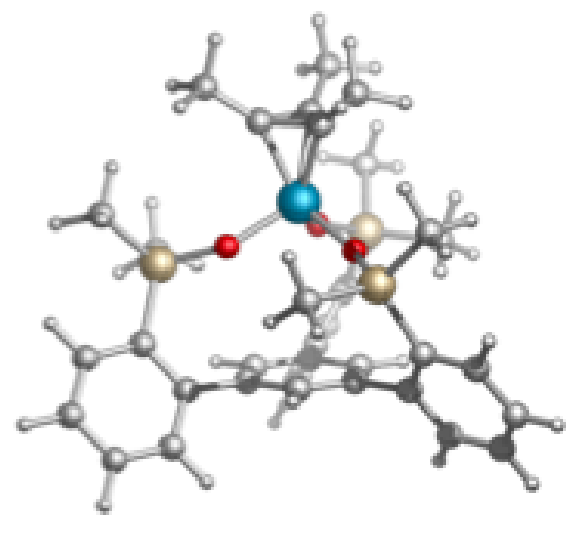

B3LYP-D3/def2TZVP-SDD(W)-CPCM(benzene)//B3LYP-

D3/def2SVP-LANL2DZ(W)-CPCM(benzene)

$\mathrm{HF}=-2559.5130429$

PBEPBE/def2TZVP-SDD(W)-CPCM(benzene)//B3LYP-D3/def2SVP-LANL2DZ(W)CPCM(benzene)

$\mathrm{HF}=-2556.8112139$

\section{[W]-B-TS}

B3LYP-D3/def2SVP-LANL2DZ(W)-CPCM(benzene)

Imaginary frequency $=-191.45 \mathrm{~cm}^{-1}$

Zero-point correction $=0.676752$ (Hartree/Particle)

Thermal correction to Energy $=0.723761$

Thermal correction to Enthalpy $=0.724705$

Thermal correction to Gibbs Free Energy= 0.598409

Sum of electronic and zero-point Energies $=-2557.419417$

Sum of electronic and thermal Energies $=-2557.372408$

Sum of electronic and thermal Enthalpies $=-2557.371464$

Sum of electronic and thermal Free Energies $=-2557.497760$

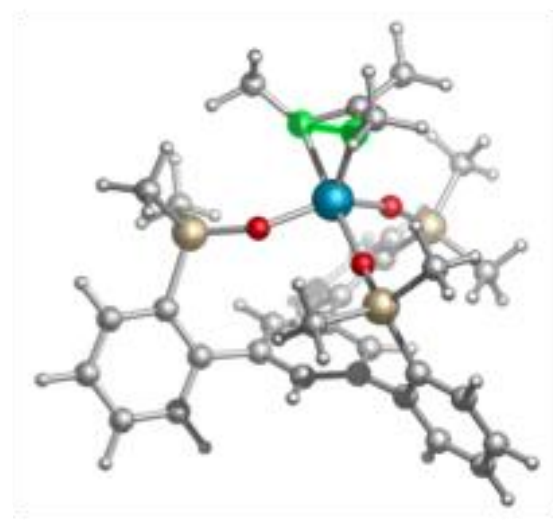

B3LYP-D3/def2TZVP-SDD(W)-CPCM(benzene)//B3LYP-D3/def2SVP-LANL2DZ(W)-

CPCM(benzene)

$\mathrm{HF}=-2559.4843308$

PBEPBE/def2TZVP-SDD(W)-CPCM(benzene)//B3LYP-D3/def2SVP-LANL2DZ(W)-

CPCM(benzene)

$\mathrm{HF}=-2556.7790799$ 


\section{[W]-D-TS}

B3LYP-D3/def2SVP-LANL2DZ(W)-CPCM(benzene)

Imaginary frequency $=-35.99 \mathrm{~cm}^{-1}$

Zero-point correction $=0.676816($ Hartree/Particle $)$

Thermal correction to Energy $=0.723480$

Thermal correction to Enthalpy $=0.724425$

Thermal correction to Gibbs Free Energy $=0.601112$

Sum of electronic and zero-point Energies $=-2557.396992$

Sum of electronic and thermal Energies $=-2557.350328$

Sum of electronic and thermal Enthalpies $=-2557.349383$

Sum of electronic and thermal Free Energies= -2557.472696

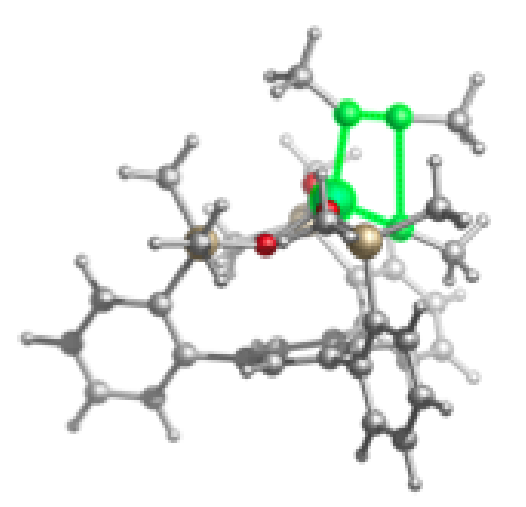

B3LYP-D3/def2TZVP-SDD(W)-CPCM(benzene)//B3LYP-D3/def2SVP-LANL2DZ(W)-

CPCM(benzene)

$\mathrm{HF}=-2559.4641124$

PBEPBE/def2TZVP-SDD(W)-CPCM(benzene)//B3LYP-D3/def2SVP-LANL2DZ(W)-

$\mathrm{CPCM}($ benzene $)$

$\mathrm{HF}=-2556.7429679$

\section{[W]-A-Ph}

B3LYP-D3/def2SVP-LANL2DZ(W)-CPCM(benzene)

Zero-point correction $=0.918026$ (Hartree/Particle $)$

Thermal correction to Energy $=0.978882$

Thermal correction to Enthalpy $=0.979826$

Thermal correction to Gibbs Free Energy= 0.816105

Sum of electronic and zero-point Energies $=-3550.979886$

Sum of electronic and thermal Energies $=-3550.919029$

Sum of electronic and thermal Enthalpies $=-3550.918085$

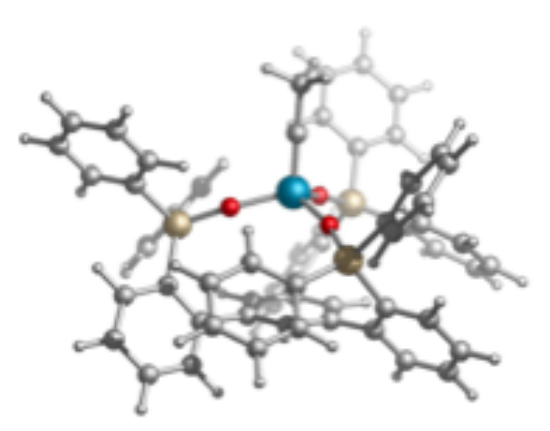

Sum of electronic and thermal Free Energies $=-3551.081806$

B3LYP-D3/def2TZVP-SDD(W)-CPCM(benzene)//B3LYP-D3/def2SVP-LANL2DZ(W)$\mathrm{CPCM}$ (benzene)

$\mathrm{HF}=-3554.3198457$

PBEPBE/def2TZVP-SDD(W)-CPCM(benzene)//B3LYP-D3/def2SVP-LANL2DZ(W)-

CPCM(benzene)

$\mathrm{HF}=-3550.3014182$ 


\section{[W]-A-TS-Ph}

B3LYP-D3/def2SVP-LANL2DZ(W)-CPCM(benzene)

Imaginary frequency $=-61.24 \mathrm{~cm}^{-1}$

Zero-point correction $=1.004403$ (Hartree/Particle)

Thermal correction to Energy $=1.070417$

Thermal correction to Enthalpy=1.071361

Thermal correction to Gibbs Free Energy $=0.900808$

Sum of electronic and zero-point Energies $=-3706.779892$

Sum of electronic and thermal Energies $=-3706.713879$

Sum of electronic and thermal Enthalpies $=-3706.712935$

Sum of electronic and thermal Free Energies $=-3706.883487$

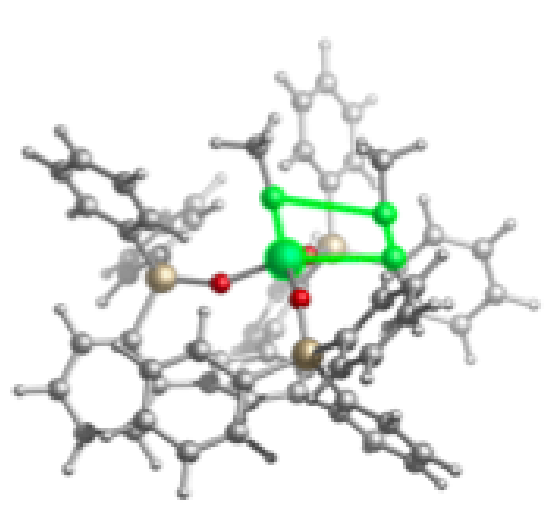

B3LYP-D3/def2TZVP-SDD(W)-CPCM(benzene)//B3LYP-D3/def2SVP-LANL2DZ(W)-

CPCM(benzene)

$\mathrm{HF}=-3710.3792583$

PBEPBE/def2TZVP-SDD(W)-CPCM(benzene)//B3LYP-D3/def2SVP-LANL2DZ(W)-

CPCM(benzene)

$\mathrm{HF}=-3706.1076251$

\section{[W]-B-Ph}

\section{B3LYP-D3/def2SVP-LANL2DZ(W)-CPCM(benzene)}

Zero-point correction $=1.006845($ Hartree/Particle $)$

Thermal correction to Energy $=1.072263$

Thermal correction to Enthalpy $=1.073207$

Thermal correction to Gibbs Free Energy $=0.904075$

Sum of electronic and zero-point Energies $=-3706.795399$

Sum of electronic and thermal Energies $=-3706.729981$

Sum of electronic and thermal Enthalpies $=-3706.729037$

Sum of electronic and thermal Free Energies= -3706.898169

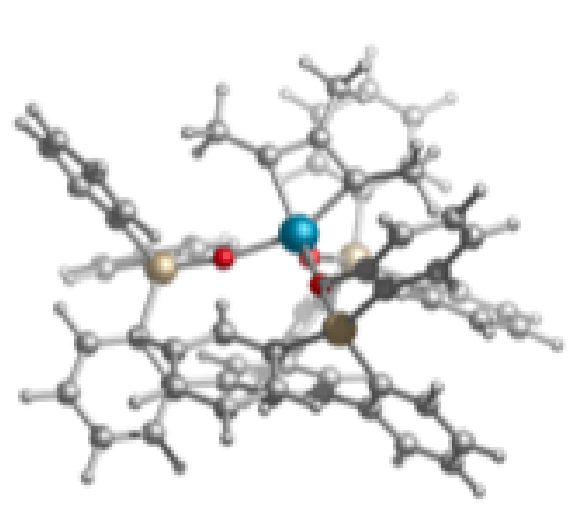

B3LYP-D3/def2TZVP-SDD(W)-CPCM(benzene)//B3LYP-

D3/def2SVP-LANL2DZ(W)-CPCM(benzene)

$\mathrm{HF}=-3710.3927507$

PBEPBE/def2TZVP-SDD(W)-CPCM(benzene)//B3LYP-D3/def2SVP-LANL2DZ(W)-

CPCM(benzene)

$\mathrm{HF}=-3706.1336769$ 
[W]-B-TS-B'-Ph

B3LYP-D3/def2SVP-LANL2DZ(W)-CPCM(benzene)

Imaginary frequency $=-115.91 \mathrm{~cm}^{-1}$

Zero-point correction=

Thermal correction to Energy=

1.005410 (Hartree/Particle)

1.070377

Thermal correction to Enthalpy=

1.071321

Thermal correction to Gibbs Free Energy=

0.902417

Sum of electronic and zero-point Energies=

$-3706.782329$

Sum of electronic and thermal Energies=

$-3706.717362$

$-3706.716418$

Sum of electronic and thermal Free Energies $=\quad-3706.885323$

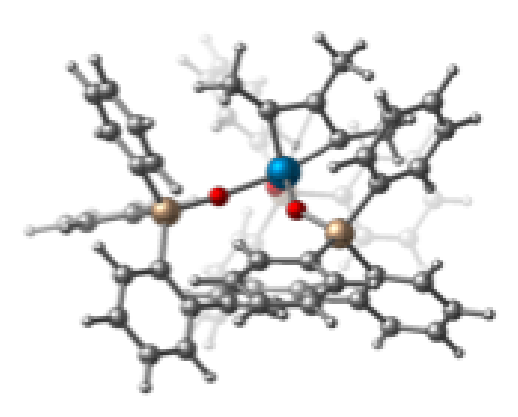

B3LYP-D3/def2TZVP-SDD(W)-CPCM(benzene)//B3LYP-D3/def2SVP-LANL2DZ(W)-

CPCM(benzene)

$\mathrm{HF}=-3710.3811163$

PBEPBE/def2TZVP-SDD(W)-CPCM(benzene)//B3LYP-D3/def2SVP-LANL2DZ(W)-

CPCM(benzene)

$\mathrm{HF}=-3706.1342496$

\section{[W]-B'-Ph}

\section{B3LYP-D3/def2SVP-LANL2DZ(W)-CPCM(benzene)}

Zero-point correction $=1.006770($ Hartree/Particle $)$

Thermal correction to Energy $=1.071942$

Thermal correction to Enthalpy $=1.072886$

Thermal correction to Gibbs Free Energy $=0.903533$

Sum of electronic and zero-point Energies $=-3706.805211$

Sum of electronic and thermal Energies $=-3706.740038$

Sum of electronic and thermal Enthalpies $=-3706.739094$

Sum of electronic and thermal Free Energies $=-3706.908447$

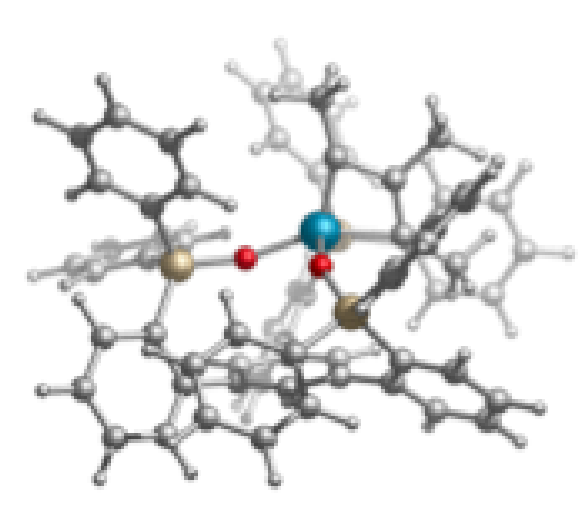

B3LYP-D3/def2TZVP-SDD(W)-CPCM(benzene)//B3LYP-D3/def2SVP-LANL2DZ(W)-

CPCM(benzene)

$\mathrm{HF}=-3710.4039999$

PBEPBE/def2TZVP-SDD(W)-CPCM(benzene)//B3LYP-D3/def2SVP-LANL2DZ(W)-

CPCM(benzene)

$\mathrm{HF}=-3706.1525639$ 


\section{[W]-C-Ph}

B3LYP-D3/def2SVP-LANL2DZ(W)-CPCM(benzene)

Zero-point correction $=1.007112($ Hartree/Particle $)$

Thermal correction to Energy $=1.072424$

Thermal correction to Enthalpy= 1.073368

Thermal correction to Gibbs Free Energy= 0.905894

Sum of electronic and zero-point Energies $=-3706.804354$

Sum of electronic and thermal Energies $=-3706.739043$

Sum of electronic and thermal Enthalpies $=-3706.738098$

Sum of electronic and thermal Free Energies $=-3706.905572$

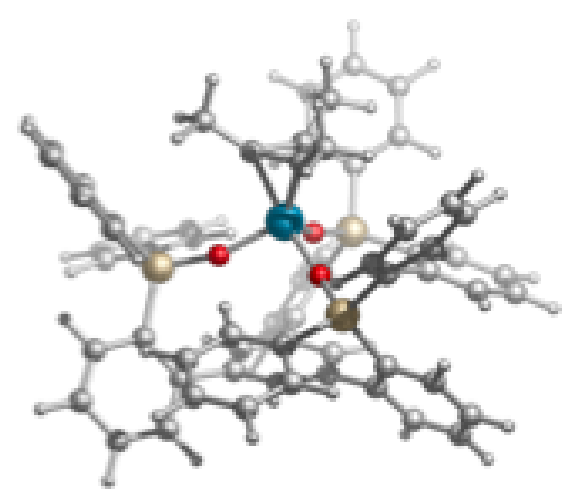

B3LYP-D3/def2TZVP-SDD(W)-CPCM(benzene)//B3LYP-D3/def2SVP-LANL2DZ(W)-

CPCM(benzene)

$\mathrm{HF}=-3710.397567$

PBEPBE/def2TZVP-SDD(W)-CPCM(benzene)//B3LYP-D3/def2SVP-LANL2DZ(W)-

CPCM(benzene)

$\mathrm{HF}=-3706.1463563$

\section{[W]-B-TS-Ph}

\section{B3LYP-D3/def2SVP-LANL2DZ(W)-CPCM(benzene)}

Imaginary frequency $=-212.31 \mathrm{~cm}^{-1}$

Zero-point correction $=1.004097($ Hartree/Particle $)$

Thermal correction to Energy $=1.069572$

Thermal correction to Enthalpy $=1.070516$

Thermal correction to Gibbs Free Energy= 0.899188

Sum of electronic and zero-point Energies $=-3706.774043$

Sum of electronic and thermal Energies $=-3706.708568$

Sum of electronic and thermal Enthalpies $=-3706.707624$

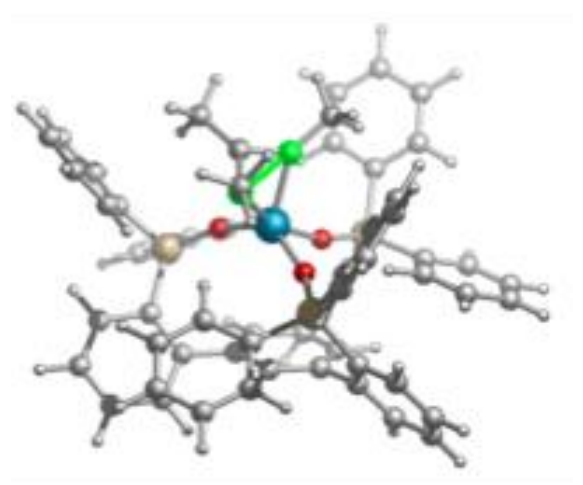

Sum of electronic and thermal Free Energies $=-3706.878951$

B3LYP-D3/def2TZVP-SDD(W)-CPCM(benzene)//B3LYP-D3/def2SVP-LANL2DZ(W)CPCM(benzene)

$\mathrm{HF}=-3710.3697736$

PBEPBE/def2TZVP-SDD(W)-CPCM(benzene)//B3LYP-D3/def2SVP-LANL2DZ(W)-

$\mathrm{CPCM}($ benzene)

$\mathrm{HF}=-3706.118118$ 


\section{[W]-D-TS-Ph}

B3LYP-D3/def2SVP-LANL2DZ(W)-CPCM(benzene)

Imaginary frequency $=-108.94 \mathrm{~cm}^{-1}$

Zero-point correction $=1.004743$ (Hartree/Particle)

Thermal correction to Energy= 1.069366

Thermal correction to Enthalpy $=1.070310$

Thermal correction to Gibbs Free Energy $=0.903823$

Sum of electronic and zero-point Energies $=-3706.721912$

Sum of electronic and thermal Energies $=-3706.657289$

Sum of electronic and thermal Enthalpies $=-3706.656345$

Sum of electronic and thermal Free Energies $=-3706.822832$

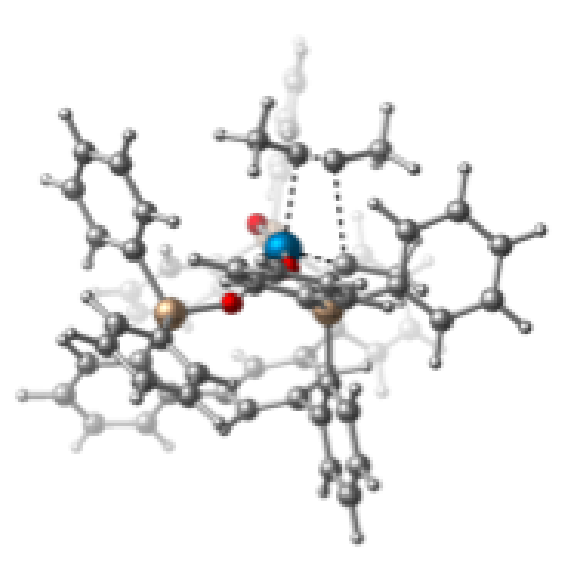

B3LYP-D3/def2TZVP-SDD(W)-CPCM(benzene)//B3LYP-D3/def2SVP-LANL2DZ(W)CPCM(benzene)

$\mathrm{HF}=-3710.3202413$

PBEPBE/def2TZVP-SDD(W)-CPCM(benzene)//B3LYP-D3/def2SVP-LANL2DZ(W)-

CPCM(benzene)

$\mathrm{HF}=-3706.0555285$

\section{[Mo]-A}

B3LYP-D3/def2SVP-LANL2DZ(Mo)-CPCM(benzene)

Zero-point correction $=0.588330($ Hartree/Particle $)$

Thermal correction to Energy $=0.631455$

Thermal correction to Enthalpy $=0.632399$

Thermal correction to Gibbs Free Energy $=0.511970$

Sum of electronic and zero-point Energies $=-2401.306719$

Sum of electronic and thermal Energies $=-2401.263594$

Sum of electronic and thermal Enthalpies $=-2401.262650$

Sum of electronic and thermal Free Energies $=-2401.383078$

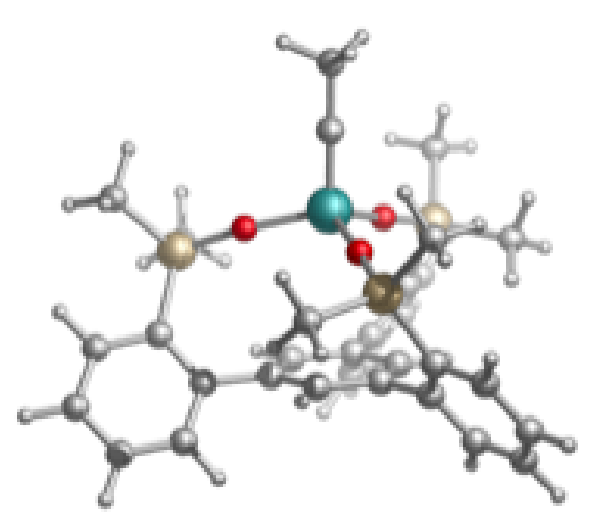

B3LYP-D3/def2TZVP-SDD(Mo)-CPCM(benzene)//B3LYP-D3/def2SVP-LANL2DZ(Mo)CPCM(benzene)

$\mathrm{HF}=-2404.5229548$

PBEPBE/def2TZVP-SDD(Mo)-CPCM(benzene)//B3LYP-D3/def2SVP-LANL2DZ(Mo)CPCM(benzene)

$\mathrm{HF}=-2402.0456315$ 


\section{[Mo]-A-TS}

B3LYP-D3/def2SVP-LANL2DZ(Mo)-CPCM(benzene)

Imaginary frequency $=-108.38 \mathrm{~cm}^{-1}$

Zero-point correction $=0.675093($ Hartree/Particle $)$

Thermal correction to Energy $=0.722735$

Thermal correction to Enthalpy $=0.723679$

Thermal correction to Gibbs Free Energy $=0.596955$

Sum of electronic and zero-point Energies $=-2557.096285$

Sum of electronic and thermal Energies $=-2557.048643$

Sum of electronic and thermal Enthalpies $=-2557.047699$

Sum of electronic and thermal Free Energies= -2557.174423

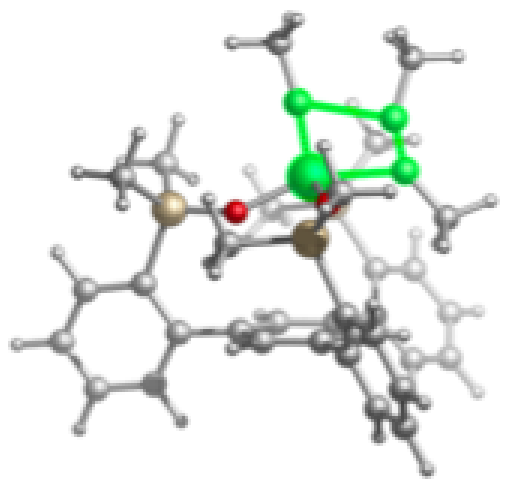

B3LYP-D3/def2TZVP-SDD(Mo)-CPCM(benzene)//B3LYP-D3/def2SVP-LANL2DZ(Mo)CPCM(benzene)

$\mathrm{HF}=-2560.5741152$

PBEPBE/def2TZVP-SDD(Mo)-CPCM(benzene)//B3LYP-D3/def2SVP-LANL2DZ(Mo)-

CPCM(benzene)

$\mathrm{HF}=-2557.8584398$

\section{[Mo]-B}

B3LYP-D3/def2SVP-LANL2DZ(Mo)-CPCM(benzene)

Zero-point correction $=0.677811$ (Hartree/Particle)

Thermal correction to Energy $=0.725151$

Thermal correction to Enthalpy $=0.726095$

Thermal correction to Gibbs Free Energy $=0.599392$

Sum of electronic and zero-point Energies $=-2557.107330$

Sum of electronic and thermal Energies $=-2557.059989$

Sum of electronic and thermal Enthalpies $=-2557.059045$

Sum of electronic and thermal Free Energies $=-2557.185748$

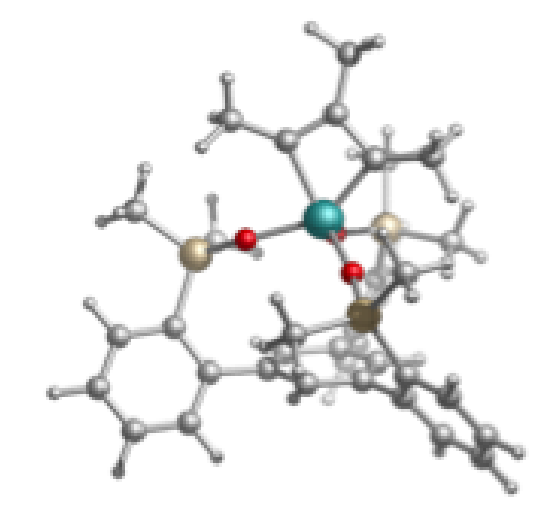

B3LYP-D3/def2TZVP-SDD(Mo)-CPCM(benzene)//B3LYP-D3/def2SVP-LANL2DZ(Mo)CPCM(benzene)

$\mathrm{HF}=-2560.5802534$

PBEPBE/def2TZVP-SDD(Mo)-CPCM(benzene)//B3LYP-D3/def2SVP-LANL2DZ(Mo)-

CPCM(benzene)

$\mathrm{HF}=-2557.871772$ 


\section{[Mo]-B-TS-B'}

B3LYP-D3/def2SVP-LANL2DZ(Mo)-CPCM(benzene)

Imaginary frequency $=-200.55 \mathrm{~cm}^{-1}$

Zero-point correction $=$

0.676296 (Hartree/Particle)

Thermal correction to Energy=

0.723269

Thermal correction to Enthalpy=

0.724213

Thermal correction to Gibbs Free Energy=

0.598902

Sum of electronic and zero-point Energies=

$-2557.092269$

Sum of electronic and thermal Energies= $-2557.045296$

Sum of electronic and thermal Enthalpies= $-2557.044352$

Sum of electronic and thermal Free Energies $=\quad-2557.169664$

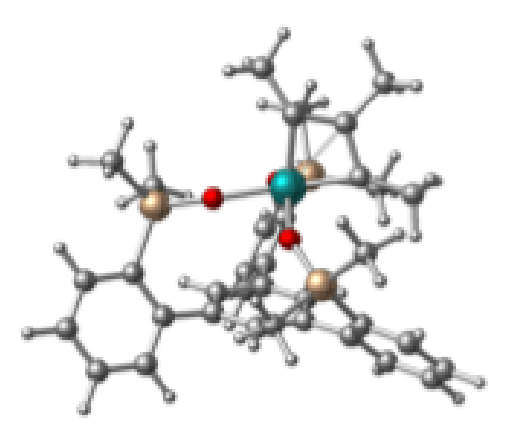

B3LYP-D3/def2TZVP-SDD(Mo)-CPCM(benzene)//B3LYP-D3/def2SVP-LANL2DZ(Mo)-

CPCM(benzene)

$\mathrm{HF}=-2560.5658088$

PBEPBE/def2TZVP-SDD(Mo)-CPCM(benzene)//B3LYP-D3/def2SVP-LANL2DZ(Mo)-

CPCM(benzene)

$\mathrm{HF}=-2557.8592625$

\section{[Mo]-B'}

\section{B3LYP-D3/def2SVP-LANL2DZ(Mo)-CPCM(benzene)}

Zero-point correction $=0.677674$ (Hartree/Particle)

Thermal correction to Energy $=0.724866$

Thermal correction to Enthalpy $=0.725810$

Thermal correction to Gibbs Free Energy $=0.599312$

Sum of electronic and zero-point Energies $=-2557.108581$

Sum of electronic and thermal Energies $=-2557.061389$

Sum of electronic and thermal Enthalpies $=-2557.060444$

Sum of electronic and thermal Free Energies $=-2557.186942$

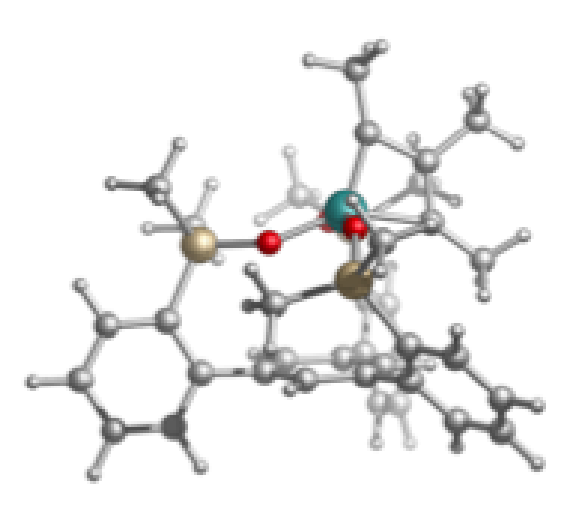

B3LYP-D3/def2TZVP-SDD(Mo)-CPCM(benzene)//B3LYP-D3/def2SVP-LANL2DZ(Mo)-

CPCM(benzene)

$\mathrm{HF}=-2560.5851268$

PBEPBE/def2TZVP-SDD(Mo)-CPCM(benzene)//B3LYP-D3/def2SVP-LANL2DZ(Mo)-

CPCM(benzene)

$\mathrm{HF}=-2557.8759407$ 


\section{[Mo]-B-TS-ent-B}

B3LYP-D3/def2SVP-LANL2DZ(Mo)-CPCM(benzene)

Imaginary frequency $=-9.36 \mathrm{~cm}^{-1}$

Zero-point correction=

Thermal correction to Energy=

0.675593 (Hartree/Particle)

0.723002

Thermal correction to Enthalpy=

0.723946

Thermal correction to Gibbs Free Energy= 0.597023

Sum of electronic and zero-point Energies $=\quad-2557.101177$

Sum of electronic and thermal Energies $=\quad-2557.053768$

Sum of electronic and thermal Enthalpies $=\quad-2557.052824$

Sum of electronic and thermal Free Energies $=\quad-2557.179746$

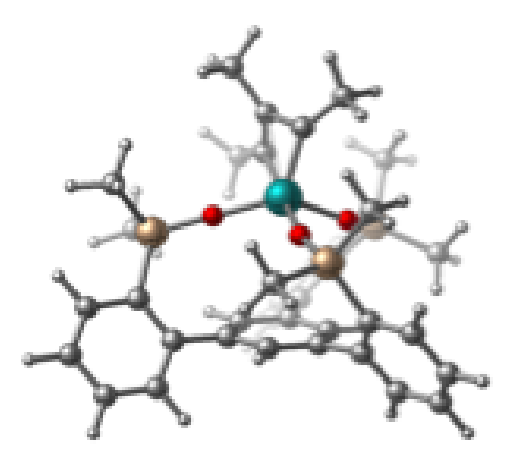

B3LYP-D3/def2TZVP-SDD(Mo)-CPCM(benzene)//B3LYP-D3/def2SVP-LANL2DZ(Mo)-

CPCM(benzene)

$\mathrm{HF}=-2560.5742476$

PBEPBE/def2TZVP-SDD(Mo)-CPCM(benzene)//B3LYP-D3/def2SVP-LANL2DZ(Mo)-

CPCM(benzene)

$\mathrm{HF}=-2557.8660984$

\section{[Mo]-C}

B3LYP-D3/def2SVP-LANL2DZ(Mo)-CPCM(benzene)

Zero-point correction $=0.678894$ (Hartree/Particle)

Thermal correction to Energy $=0.725931$

Thermal correction to Enthalpy $=0.726875$

Thermal correction to Gibbs Free Energy $=0.601238$

Sum of electronic and zero-point Energies $=-2557.123525$

Sum of electronic and thermal Energies $=-2557.076489$

Sum of electronic and thermal Enthalpies $=-2557.075544$

Sum of electronic and thermal Free Energies $=-2557.201181$

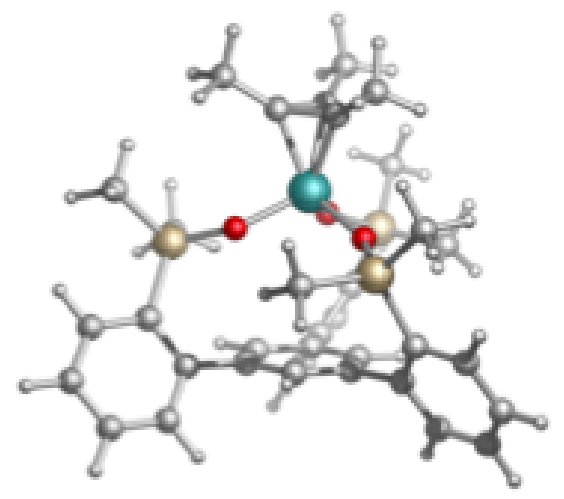

B3LYP-D3/def2TZVP-SDD(Mo)-CPCM(benzene)//B3LYP-D3/def2SVP-LANL2DZ(Mo)-

CPCM(benzene)

$\mathrm{HF}=-2560.5923248$

PBEPBE/def2TZVP-SDD(Mo)-CPCM(benzene)//B3LYP-D3/def2SVP-LANL2DZ(Mo)CPCM(benzene)

$\mathrm{HF}=-2557.8885247$ 


\section{[Mo]-B-TS}

B3LYP-D3/def2SVP-LANL2DZ(Mo)-CPCM(benzene)

Imaginary frequency $=-179.76 \mathrm{~cm}^{-1}$

Zero-point correction $=0.676178($ Hartree/Particle $)$

Thermal correction to Energy $=0.723241$

Thermal correction to Enthalpy $=0.724185$

Thermal correction to Gibbs Free Energy $=0.598258$

Sum of electronic and zero-point Energies $=-2557.086727$

Sum of electronic and thermal Energies $=-2557.039664$

Sum of electronic and thermal Enthalpies $=-2557.038719$

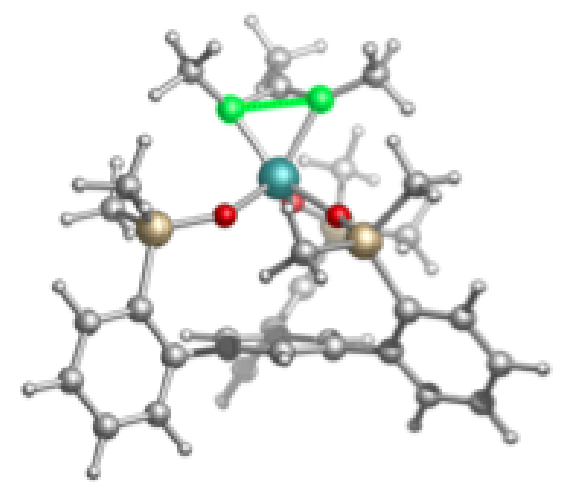

Sum of electronic and thermal Free Energies $=-2557.164647$

B3LYP-D3/def2TZVP-SDD(Mo)-CPCM(benzene)//B3LYP-D3/def2SVP-LANL2DZ(Mo)CPCM(benzene)

$\mathrm{HF}=-2560.5594384$

PBEPBE/def2TZVP-SDD(Mo)-CPCM(benzene)//B3LYP-D3/def2SVP-LANL2DZ(Mo)-

CPCM(benzene)

$\mathrm{HF}=-2557.8535094$

\section{[Mo]-D-TS}

B3LYP-D3/def2SVP-LANL2DZ(Mo)-CPCM(benzene)

Imaginary frequency $=-249.08 \mathrm{~cm}^{-1}$

Zero-point correction $=0.675819$ (Hartree/Particle $)$

Thermal correction to Energy $=0.722782$

Thermal correction to Enthalpy $=0.723726$

Thermal correction to Gibbs Free Energy $=0.599520$

Sum of electronic and zero-point Energies $=-2557.055119$

Sum of electronic and thermal Energies $=-2557.008156$

Sum of electronic and thermal Enthalpies $=-2557.007212$

Sum of electronic and thermal Free Energies $=-2557.131419$

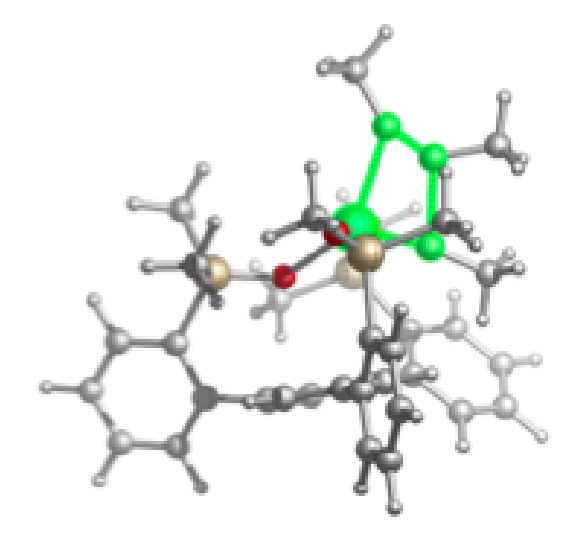

B3LYP-D3/def2TZVP-SDD(Mo)-CPCM(benzene)//B3LYP-D3/def2SVP-LANL2DZ(Mo)-

CPCM(benzene)

$\mathrm{HF}=-2560.5287423$

PBEPBE/def2TZVP-SDD(Mo)-CPCM(benzene)//B3LYP-D3/def2SVP-LANL2DZ(Mo)-

CPCM(benzene)

$\mathrm{HF}=-2557.816809$ 


\section{[Mo]-A-Ph}

B3LYP-D3/def2SVP-LANL2DZ(Mo)-CPCM(benzene)

Zero-point correction $=0.917179($ Hartree/Particle $)$

Thermal correction to Energy $=0.978141$

Thermal correction to Enthalpy $=0.979086$

Thermal correction to Gibbs Free Energy= 0.814927

Sum of electronic and zero-point Energies $=-3550.648228$

Sum of electronic and thermal Energies $=-3550.587266$

Sum of electronic and thermal Enthalpies $=-3550.586322$

Sum of electronic and thermal Free Energies $=-3550.750480$

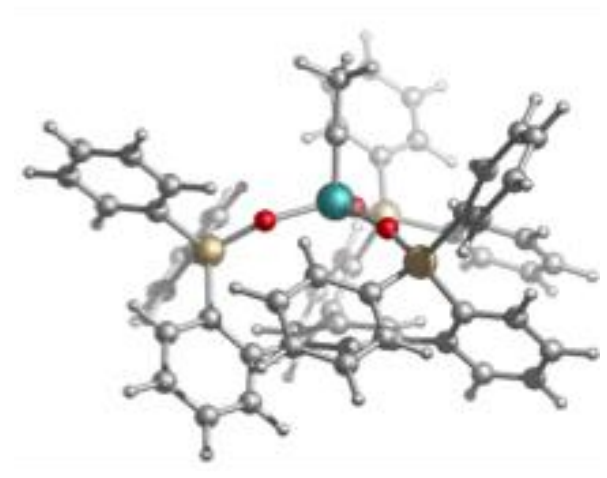

B3LYP-D3/def2TZVP-SDD(Mo)-CPCM(benzene)//B3LYP-D3/def2SVP-LANL2DZ(Mo)CPCM(benzene)

$\mathrm{HF}=-3555.396031$

PBEPBE/def2TZVP-SDD(Mo)-CPCM(benzene)//B3LYP-D3/def2SVP-LANL2DZ(Mo)-

CPCM(benzene)

$\mathrm{HF}=-3551.3819436$

\section{[Mo]-A-TS-Ph}

\section{B3LYP-D3/def2SVP-LANL2DZ(Mo)-CPCM(benzene)}

Imaginary frequency $=-96.26 \mathrm{~cm}^{-1}$

Zero-point correction $=1.004448$ (Hartree/Particle)

Thermal correction to Energy $=1.069819$

Thermal correction to Enthalpy $=1.070764$

Thermal correction to Gibbs Free Energy $=0.901741$

Sum of electronic and zero-point Energies= -3706.439514

Sum of electronic and thermal Energies $=-3706.374143$

Sum of electronic and thermal Enthalpies $=-3706.373198$

Sum of electronic and thermal Free Energies= -3706.542221

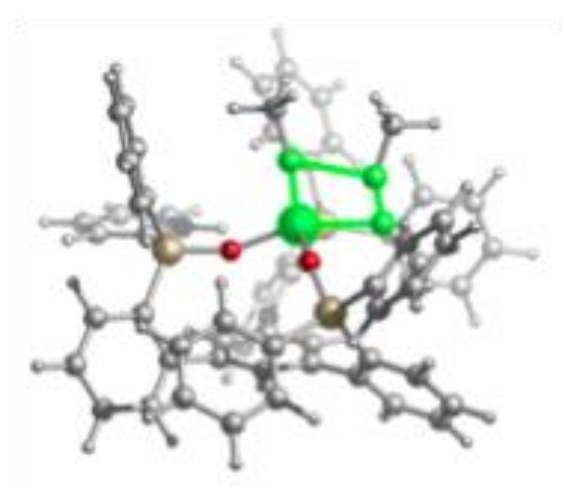

B3LYP-D3/def2TZVP-SDD(Mo)-CPCM(benzene)//B3LYP-D3/def2SVP-LANL2DZ(Mo)-

$\mathrm{CPCM}($ benzene)

$\mathrm{HF}=-3711.4494332$

PBEPBE/def2TZVP-SDD(Mo)-CPCM(benzene)//B3LYP-D3/def2SVP-LANL2DZ(Mo)-

CPCM(benzene)

$\mathrm{HF}=-3707.1943604$ 


\section{[Mo]-B-Ph}

B3LYP-D3/def2SVP-LANL2DZ(Mo)-CPCM(benzene)

Zero-point correction $=1.006303$ (Hartree/Particle)

Thermal correction to Energy $=1.071843$

Thermal correction to Enthalpy $=1.072787$

Thermal correction to Gibbs Free Energy= 0.904069

Sum of electronic and zero-point Energies $=-3706.455213$

Sum of electronic and thermal Energies $=-3706.389672$

Sum of electronic and thermal Enthalpies $=-3706.388728$

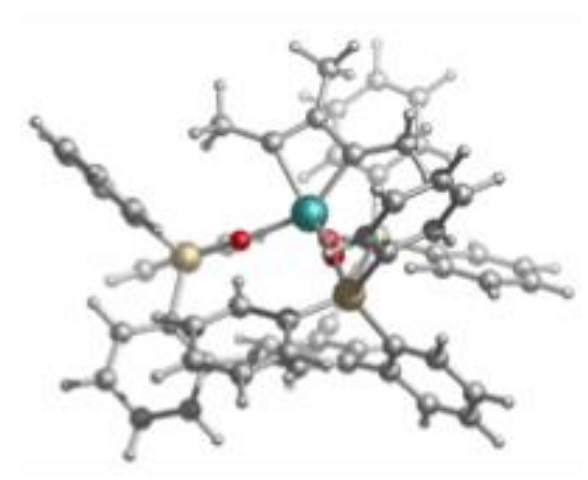

Sum of electronic and thermal Free Energies $=-3706.557447$

B3LYP-D3/def2TZVP-SDD(Mo)-CPCM(benzene)//B3LYP-D3/def2SVP-LANL2DZ(Mo)-

CPCM(benzene)

$\mathrm{HF}=-3711.459603$

PBEPBE/def2TZVP-SDD(Mo)-CPCM(benzene)//B3LYP-D3/def2SVP-LANL2DZ(Mo)-

CPCM(benzene)

$\mathrm{HF}=-3707.2048744$

\section{[Mo]-B-TS-B'-Ph}

B3LYP-D3/def2SVP-LANL2DZ(Mo)-CPCM(benzene)

Imaginary frequency $=-211.97 \mathrm{~cm}^{-1}$

Zero-point correction $=$

1.004988 (Hartree/Particle)

Thermal correction to Energy=

1.070033

Thermal correction to Enthalpy= 1.070977

Thermal correction to Gibbs Free Energy=

0.901728

Sum of electronic and zero-point Energies=

$-3706.433662$

Sum of electronic and thermal Energies=

$-3706.368617$

Sum of electronic and thermal Enthalpies=

$-3706.367673$

Sum of electronic and thermal Free Energies=

$-3706.536923$

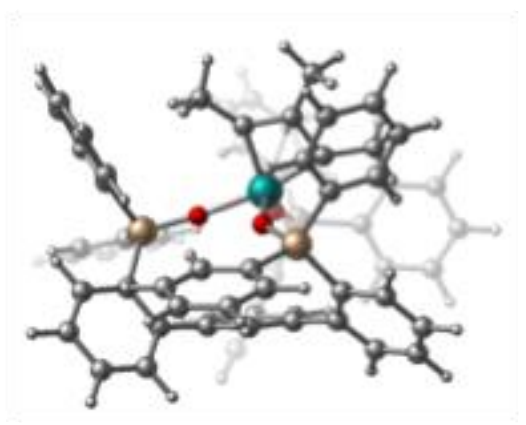

B3LYP-D3/def2TZVP-SDD(Mo)-CPCM(benzene)//B3LYP-D3/def2SVP-LANL2DZ(Mo)CPCM(benzene)

$\mathrm{HF}=-3711.4414724$

PBEPBE/def2TZVP-SDD(Mo)-CPCM(benzene)//B3LYP-D3/def2SVP-LANL2DZ(Mo)CPCM(benzene) $\mathrm{HF}=-3707.1987306$ 


\section{[Mo]-B'-Ph}

B3LYP-D3/def2SVP-LANL2DZ(Mo)-CPCM(benzene)

Zero-point correction $=1.005955$ (Hartree/Particle)

Thermal correction to Energy= 1.071363

Thermal correction to Enthalpy $=1.072308$

Thermal correction to Gibbs Free Energy $=0.902130$

Sum of electronic and zero-point Energies $=-3706.454986$

Sum of electronic and thermal Energies $=-3706.389578$

Sum of electronic and thermal Enthalpies $=-3706.388634$

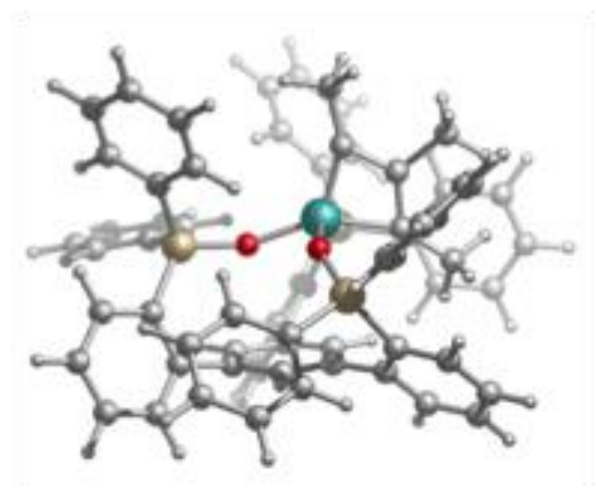

Sum of electronic and thermal Free Energies $=-3706.558812$

B3LYP-D3/def2TZVP-SDD(Mo)-CPCM(benzene)//B3LYP-D3/def2SVP-LANL2DZ(Mo)CPCM(benzene)

$\mathrm{HF}=-3711.4630733$

PBEPBE/def2TZVP-SDD(Mo)-CPCM(benzene)//B3LYP-D3/def2SVP-LANL2DZ(Mo)-

CPCM(benzene)

$\mathrm{HF}=-3707.2163281$

\section{[Mo]-C-Ph}

B3LYP-D3/def2SVP-LANL2DZ(Mo)-CPCM(benzene)

Zero-point correction $=1.006905$ (Hartree/Particle)

Thermal correction to Energy $=1.072289$

Thermal correction to Enthalpy= 1.073233

Thermal correction to Gibbs Free Energy $=0.905359$

Sum of electronic and zero-point Energies $=-3706.475869$

Sum of electronic and thermal Energies $=-3706.410485$

Sum of electronic and thermal Enthalpies $=-3706.409541$

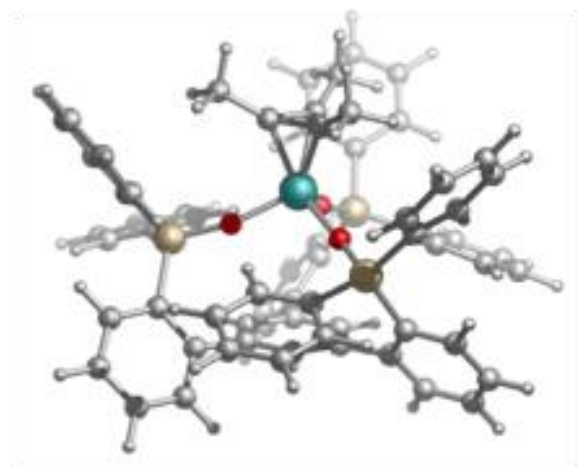

Sum of electronic and thermal Free Energies= -3706.577415

B3LYP-D3/def2TZVP-SDD(Mo)-CPCM(benzene)//B3LYP-D3/def2SVP-LANL2DZ(Mo)CPCM(benzene)

$\mathrm{HF}=-3711.4760852$

PBEPBE/def2TZVP-SDD(Mo)-CPCM(benzene)//B3LYP-D3/def2SVP-LANL2DZ(Mo)-

CPCM(benzene)

$\mathrm{HF}=-3707.227112$ 


\section{[Mo]-B-TS}

B3LYP-D3/def2SVP-LANL2DZ(Mo)-CPCM(benzene)

Imaginary frequency $=-190.20 \mathrm{~cm}^{-1}$

Zero-point correction $=1.003942($ Hartree/Particle $)$

Thermal correction to Energy= 1.069519

Thermal correction to Enthalpy $=1.070463$

Thermal correction to Gibbs Free Energy $=0.899195$

Sum of electronic and zero-point Energies $=-3706.438866$

Sum of electronic and thermal Energies $=-3706.373289$

Sum of electronic and thermal Enthalpies $=-3706.372345$

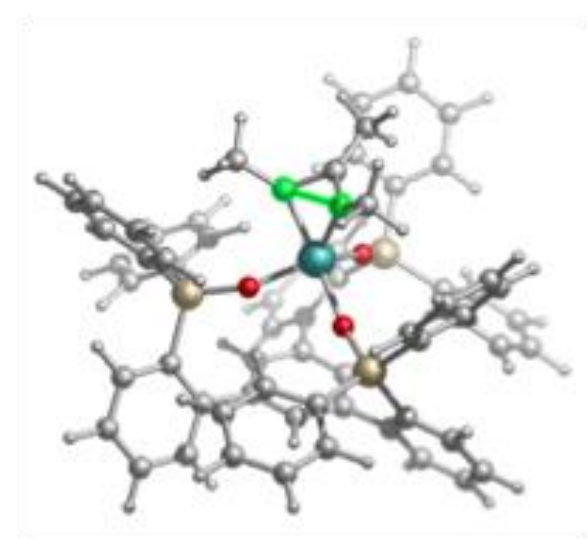

Sum of electronic and thermal Free Energies $=-3706.543613$

B3LYP-D3/def2TZVP-SDD(Mo)-CPCM(benzene)//B3LYP-D3/def2SVP-LANL2DZ(Mo)CPCM(benzene)

$\mathrm{HF}=-3711.4425462$

PBEPBE/def2TZVP-SDD(Mo)-CPCM(benzene)//B3LYP-D3/def2SVP-LANL2DZ(Mo)-

CPCM(benzene)

$\mathrm{HF}=-3707.1925016$

\section{[Mo]-D-TS-Ph}

B3LYP-D3/def2SVP-LANL2DZ(Mo)-CPCM(benzene)

Imaginary frequency $=-35.52 \mathrm{~cm}^{-1}$

Zero-point correction $=1.004363$ (Hartree/Particle)

Thermal correction to Energy $=1.068922$

Thermal correction to Enthalpy $=1.069866$

Thermal correction to Gibbs Free Energy $=0.904287$

Sum of electronic and zero-point Energies $=-3706.389618$

Sum of electronic and thermal Energies $=-3706.325059$

Sum of electronic and thermal Enthalpies $=-3706.324115$

Sum of electronic and thermal Free Energies $=-3706.489694$

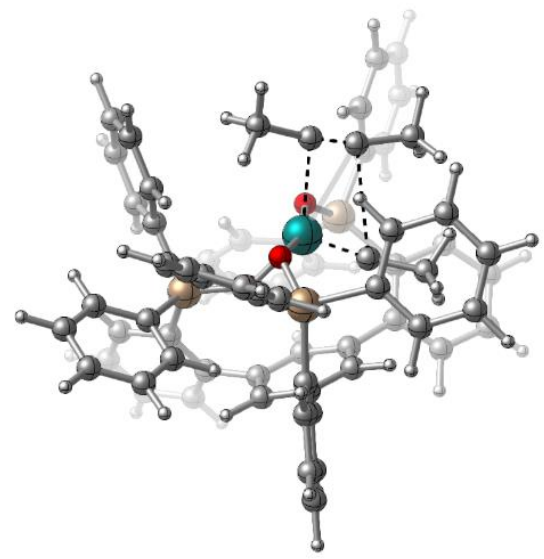

B3LYP-D3/def2TZVP-SDD(Mo)-CPCM(benzene)//B3LYP-D3/def2SVP-LANL2DZ(Mo)-

CPCM(benzene)

$\mathrm{HF}=-3711.4375019$

PBEPBE/def2TZVP-SDD(Mo)-CPCM(benzene)//B3LYP-D3/def2SVP-LANL2DZ(Mo)CPCM(benzene)

$\mathrm{HF}=-3707.1314337$ 
[W]-Ar'

B3LYP-D3/def2SVP-LANL2DZ(W)-CPCM(benzene)

Zero-point correction=

Thermal correction to Energy=

Thermal correction to Enthalpy=

Thermal correction to Gibbs Free Energy=

Sum of electronic and zero-point Energies=

Sum of electronic and thermal Energies=

Sum of electronic and thermal Enthalpies=

Sum of electronic and thermal Free Energies=
0.642570 (Hartree/Particle)

0.688668

0.689612

0.559953

$-2593.202538$

$-2593.156440$

$-2593.155496$

$-2593.285155$

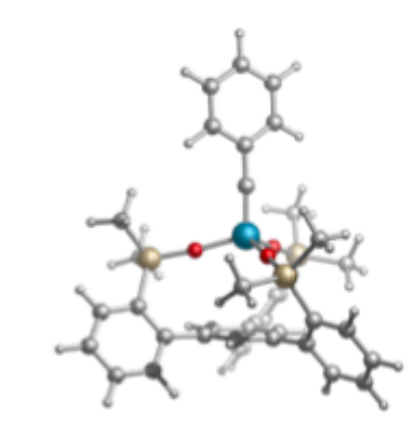

B3LYP-D3/def2TZVP-SDD(W)-CPCM(benzene)//B3LYP-D3/def2SVP-LANL2DZ(W)CPCM(benzene)

$\mathrm{HF}=-2595.2653957$

\section{[W]-Ar'-TS}

B3LYP-D3/def2SVP-LANL2DZ(W)-CPCM(benzene)

Imaginary frequency $=-76.91 \mathrm{~cm}^{-1}$

Zero-point correction $=$

0.730082 (Hartree/Particle)

Thermal correction to Energy=

Thermal correction to Enthalpy=

0.780361

0.781305

Thermal correction to Gibbs Free Energy $=0.647718$

Sum of electronic and zero-point Energies $=\quad-2749.007213$

Sum of electronic and thermal Energies=

$-2748.956934$

Sum of electronic and thermal Enthalpies=

$-2748.955989$

Sum of electronic and thermal Free Energies=

$-2749.089576$

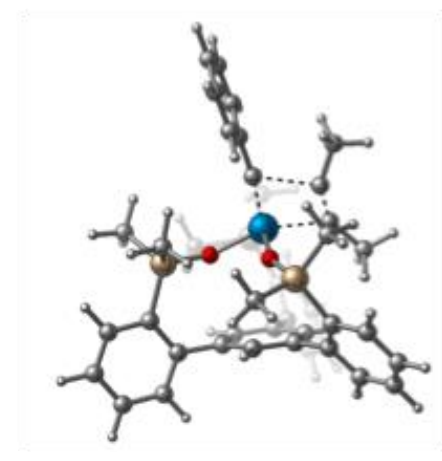

B3LYP-D3/def2TZVP-SDD(W)-CPCM(benzene)//B3LYP-D3/def2SVP-LANL2DZ(W)CPCM(benzene)

$\mathrm{HF}=-2751.3314256$

\section{[W]-C'}

\section{B3LYP-D3/def2SVP-LANL2DZ(W)-CPCM(benzene)}

Zero-point correction $=$

Thermal correction to Energy=

Thermal correction to Enthalpy=

Thermal correction to Gibbs Free Energy=

Sum of electronic and zero-point Energies=

Sum of electronic and thermal Energies=

Sum of electronic and thermal Enthalpies=

Sum of electronic and thermal Free Energies=
0.731396 (Hartree/Particle)

0.782017

0.782961

0.647715

$-2749.018238$

$-2748.967617$

$-2748.966673$

$-2749.101919$

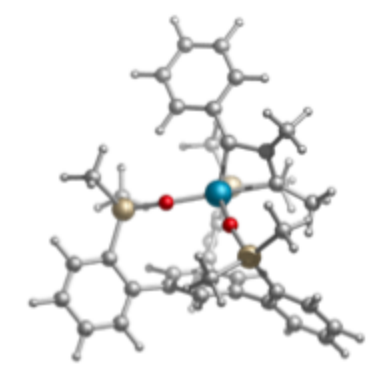


B3LYP-D3/def2TZVP-SDD(W)-CPCM(benzene)//B3LYP-D3/def2SVP-LANL2DZ(W)CPCM(benzene)

$\mathrm{HF}=-2751.3378612$

\section{[W]-ent-C}

B3LYP-D3/def2SVP-LANL2DZ(W)-CPCM(benzene)

Zero-point correction $=$

Thermal correction to Energy=

0.731817 (Hartree/Particle)

Thermal correction to Enthalpy=

0.782026

Thermal correction to Gibbs Free Energy=

0.782970

Sum of electronic and zero-point Energies=

0.650533

Sum of electronic and thermal Energies=

$-2749.028774$

$-2748.978566$

Sum of electronic and thermal Enthalpies=

$-2748.977621$

Sum of electronic and thermal Free Energies $=\quad-2749.110058$

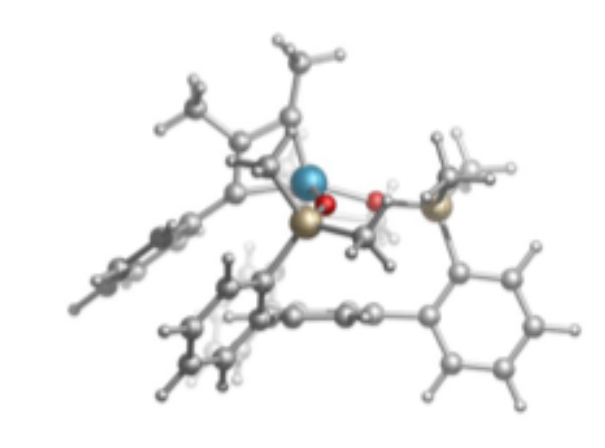

B3LYP-D3/def2TZVP-SDD(W)-CPCM(benzene)//B3LYP-D3/def2SVP-LANL2DZ(W)CPCM(benzene)

$\mathrm{HF}=-2751.3500929$

\section{[W]-F'-TS}

B3LYP-D3/def2SVP-LANL2DZ(W)-CPCM(benzene)

Imaginary frequency $=-55.06 \mathrm{~cm}^{-1}$

Zero-point correction=

Thermal correction to Energy=

0.728913 (Hartree/Particle)

Thermal correction to Enthalpy=

0.780084

Thermal correction to Gibbs Free Energy $=0.645967$

Sum of electronic and zero-point Energies $=\quad-2749.003492$

Sum of electronic and thermal Energies= $\quad-2748.952322$

Sum of electronic and thermal Enthalpies $=\quad-2748.951378$

Sum of electronic and thermal Free Energies $=\quad-2749.086439$

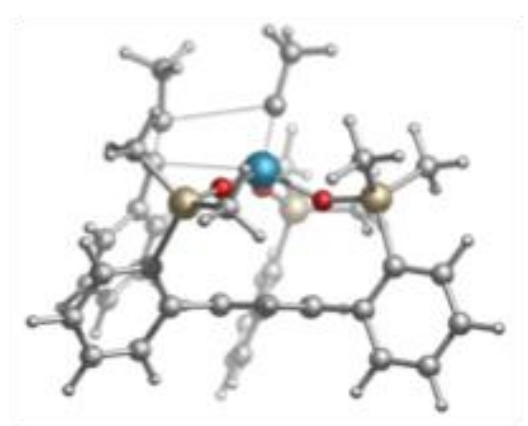

B3LYP-D3/def2TZVP-SDD(W)-CPCM(benzene)//B3LYP-D3/def2SVP-LANL2DZ(W)-

CPCM(benzene)

$\mathrm{HF}=-2751.325516$ 
[Mo]-Ar'

B3LYP-D3/def2SVP-LANL2DZ(Mo)-CPCM(benzene)

Zero-point correction $=$

0.642472

(Hartree/Particle)

Thermal correction to Energy $=\quad 0.688528$

Thermal correction to Enthalpy $=\quad 0.689472$

Thermal correction to Gibbs Free Energy= $\quad 0.560209$

Sum of electronic and zero-point Energies $=\quad-2592.869563$

Sum of electronic and thermal Energies= $-2592.823507$

$-2592.822563$

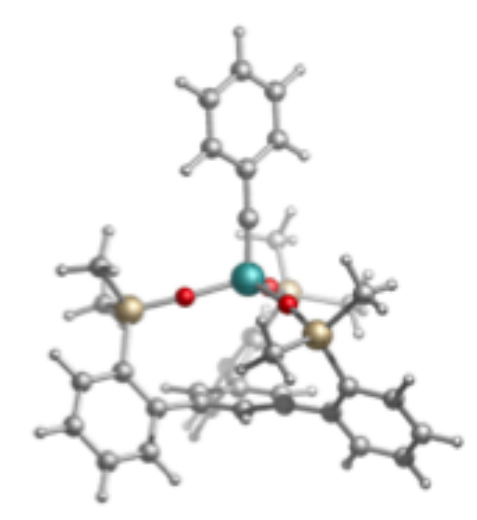

Sum of electronic and thermal Enthalpies=

$-2592.951826$

B3LYP-D3/def2TZVP-SDD(Mo)-CPCM(benzene)//B3LYP-D3/def2SVP-LANL2DZ(Mo)-

CPCM(benzene)

$\mathrm{HF}=-2596.3419656$

[Mo]-Ar'-TS

B3LYP-D3/def2SVP-LANL2DZ(Mo)-CPCM(benzene)

Imaginary frequency $=-97.67 \mathrm{~cm}^{-1}$

Zero-point correction=

Thermal correction to Energy=

0.729668 (Hartree/Particle)

0.780046

Thermal correction to Enthalpy=

0.780990

Thermal correction to Gibbs Free Energy $=0.646883$

Sum of electronic and zero-point Energies $=\quad-2748.659511$

Sum of electronic and thermal Energies=

$-2748.609133$

$\begin{array}{ll}\text { Sum of electronic and thermal Enthalpies }= & -2748.608188 \\ \text { Sum of electronic and thermal Free Energies }= & -2748.742296\end{array}$

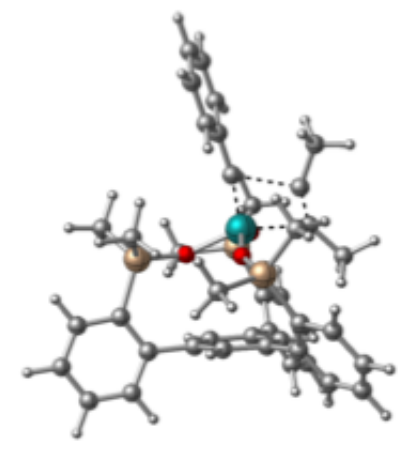

B3LYP-D3/def2TZVP-SDD(Mo)-CPCM(benzene)//B3LYP-D3/def2SVP-LANL2DZ(Mo)$\mathrm{CPCM}($ benzene)

$\mathrm{HF}=-2752.3941359$

\section{[Mo]-C'}

B3LYP-D3/def2SVP-LANL2DZ(Mo)-CPCM(benzene)

Zero-point correction=

Thermal correction to Energy=

Thermal correction to Enthalpy=

Thermal correction to Gibbs Free Energy=

Sum of electronic and zero-point Energies=

Sum of electronic and thermal Energies=

Sum of electronic and thermal Enthalpies=

Sum of electronic and thermal Free Energies=
0.731316 (Hartree/Particle)

0.781840

0.782784

0.648509

$-2748.672521$

$-2748.621997$

$-2748.621053$

$-2748.755327$

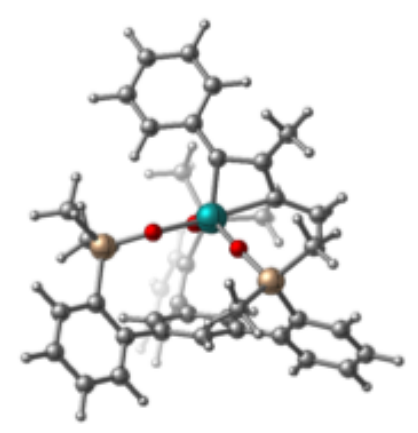


B3LYP-D3/def2TZVP-SDD(Mo)-CPCM(benzene)//B3LYP-D3/def2SVP-LANL2DZ(Mo)CPCM(benzene)

$\mathrm{HF}=-2752.4017595$

\section{[Mo]-C'-ent}

B3LYP-D3/def2SVP-LANL2DZ(Mo)-CPCM(benzene)

Zero-point correction $=$

Thermal correction to Energy=

Thermal correction to Enthalpy=

Thermal correction to Gibbs Free Energy=

Sum of electronic and zero-point Energies $=$

Sum of electronic and thermal Energies=

Sum of electronic and thermal Enthalpies=

Sum of electronic and thermal Free Energies=
0.727843 (Hartree/Particle)

0.780565

0.781509

0.639155

$-2748.668908$

$-2748.616186$

$-2748.615242$

$-2748.757596$

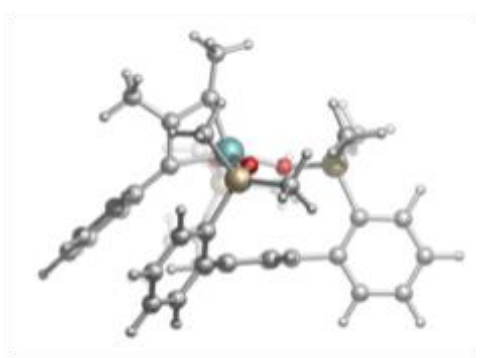

B3LYP-D3/def2TZVP-SDD(Mo)-CPCM(benzene)//B3LYP-D3/def2SVP-LANL2DZ(Mo)CPCM(benzene)

$\mathrm{HF}=-2752.3977942$

\section{[Mo]-F'-TS}

B3LYP-D3/def2SVP-LANL2DZ(Mo)-CPCM(benzene)

Imaginary frequency $=-101.61 \mathrm{~cm}^{-1}$

Zero-point correction=

0.729075 (Hartree/Particle)

Thermal correction to Energy=

0.779561

Thermal correction to Enthalpy=

0.780506

Thermal correction to Gibbs Free Energy $=\quad 0.647665$

Sum of electronic and zero-point Energies $=\quad-2748.663139$

Sum of electronic and thermal Energies $=\quad-2748.612653$

Sum of electronic and thermal Enthalpies= $\quad-2748.611708$

Sum of electronic and thermal Free Energies $=\quad-2748.744549$

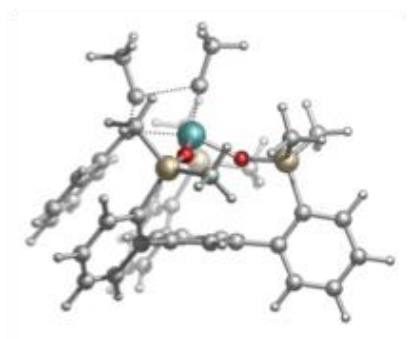

B3LYP-D3/def2TZVP-SDD(Mo)-CPCM(benzene)//B3LYP-D3/def2SVP-LANL2DZ(Mo)CPCM(benzene)

$\mathrm{HF}=-2752.3955648$ 


\section{$\underline{\text { References }}$}

1. Trawny, D.; Quennet, M.; Rades, N.; Lentz, D.; Paulus, B.; Reissig, H., Eur. J. Org. Chem. 2015, 4667-4674.

2. Gao, S.; Wu, Z.; Fang, X.; Lin, A.; Yao, H., Org. Lett. 2016, 18, 3906-3909.

3. (a) Haberlag, B.; Wu, X.; Brandhorst, K.; Grunenberg, J.; Daniliuc, C. G.; Jones, P. G.; Tamm, M., Chem. Eur. J. 2010, 16, 8868-8877. (b) Haberlag, B., Freytag, M., Daniliuc, C.G., Jones, P.G. and Tamm, M., Angew. Chem. Int. Ed. 2012, 51, 13019-13022.

4. Bittner, C.; Ehrhorn, H.; Bockfeld, D.; Brandhorst, K.; Tamm, M., Organometallics 2017, 36, 3398-3406.

5. Thompson, R. R.; Rotella, M. E.; Du, P.; Zhou, X.; Fronczek, F. R.; Kumar, R.; Gutierrez, O.; Lee, S., Organometallics 2019, 38, 4054-4059.

6. SAINT; Bruker AXS, Inc.: Madison, WI, USA. 2009.

7. Sheldrick, G. M., SADABS,: 2007, University of Gottingen, Germany.

8. Sheldrick, G., SHELXT - Integrated space-group and crystal-structure determination. Acta Cryst. 2015, A71, 3-8.

9. Sheldrick, G. M. (2014). SHELXL-2014. University of Gottingen, Germany.

10. a) Becke, A. D. Density-functional thermochemistry. III. The role of exact exchange. $J$. Chem. Phys., 1993, 98, 5648-5652. b) Grimme, S. Semiempirical GGA-type density functional constructed with a long-range dispersion correction. J. Comp. Chem., 2006, 27, 1787-1799. c) Weigend, F.; Ahlrichs, R. Balanced basis sets of split valence, triple zeta valence and quadruple zeta valence quality for $\mathrm{H}$ to $\mathrm{Rn}$ : Design and assessment of accuracy. Phys. Chem. Chem. Phys., 2005, 7, 3297-3305. d) Hay, P. J.; Wadt, W. R. Ab initio effective core potentials for molecular calculations - potentials for the transitionmetal atoms Sc to Hg. J. Chem. Phys., 1985, 82, 270-283.

11. Tomasi, J.; Mennucci, B.; Cammi, R. Quantum mechanical continuum solvation models. Chem. Rev., 2005, 105, 2999-3093.

12. a) Weigend, F. Accurate Coulomb-fitting basis sets for $\mathrm{H}$ to Rn. Phys. Chem. Chem. Phys., 2006, 8, 1057-1065. b) Fuentealba, P.; Preuss, H.; Stoll, H.; Szentpály, L. V. A Proper Account of Core-polarization with Pseudopotentials - Single Valence-Electron Alkali Compounds. Chem. Phys. Lett., 1982, 89, 418-22.

13. Mulliken, R. S., Electronic Population Analysis on LCAO-MO Molecular Wave Functions. I. J. Chem. Phys. 1955, 23, 1833-1840.

14. CYLview, 1.0b; Legault, C. Y., Université de Sherbrooke, 2009 (http://www.cylview.org)

15. Lu, T.; Chen, F., Multiwfn: A multifunctional wavefunction analyzer. J. Comp. Chem. 2012, 33, 580-592.

16. Humphrey, W.; Dalke, A.; Schulten, K., VMD - Visual Molecular Dynamics. J. Mol. Graphics 1996, 14, 33-38.

17. (a) Horn, P. R.; Head-Gordon, M. Alternative definitions of the frozen energy in energy decomposition analysis of density functional theory calculations. J. Chem. Phys. 2016, 144, 084118. (b) Horn, P. R.; Mao, Y.; Head-Gordon, M. Defining the contributions of permanent electrostatics, Pauli repulsion, and dispersion in density functional theory calculations of intermolecular interaction energies. J. Chem. Phys. 2016, 144, 114107. (c) Horn, P. R.; Mao, Y.; Head-Gordon, M. Probing non-covalent interactions with a second- 
generation energy decomposition analysis using absolutely localized molecular orbitals. Phys. Chem. Chem. Phys. 2016, 18, 23067-23079.

18. Shao, Y.; Gan, Z.; Epifanovsky, E.; Gilbert, A. T. B.; Wormit, M.; Kussmann, J.; Lange, A. W.; Behn, A.; Deng, J.; Feng, X.; Ghosh, D.; Goldey, M.; Horn, P. R.; Jacobson, L. D.; Kaliman, I.; Khaliullin, R. Z.; Kus, T.; Landau, A.; Liu, J.; Proynov, E. I.; Rhee, Y. M.; Richard, R. M.; Rohrdanz, M. A.; Steele, R. P.; Sundstrom, E. J.; Woodcock, H. L.; Zimmerman, P. M.; Zuev, D.; Albrecht, B.; Alguire, E.; Austin, B.; Beran, G. J. O.; Bernard, Y. A.; Berquist, E.; Brandhorst, K.; Bravaya, K. B.; Brown, S. T.; Casanova, D.; Chang, C.-M.; Chen, Y.; Chien, S. H.; Closser, K. D.; Crittenden, D. L.; Diedenhofen, M.; DiStasio, R. A.; Do, H.; Dutoi, A. D.; Edgar, R. G.; Fatehi, S.; Fusti-Molnar, L.; Ghysels, A.; Golubeva-Zadorozhnaya, A.; Gomes, J.; Hanson-Heine, M. W. D.; Harbach, P. H. P.; Hauser, A. W.; Hohenstein, E. G.; Holden, Z. C.; Jagau, T.-C.; Ji, H.; Kaduk, B.; Khistyaev, K.; Kim, J.; Kim, J.; King, R. A.; Klunzinger, P.; Kosenkov, D.; Kowalczyk, T.; Krauter, C. M.; Lao, K. U.; Laurent, A. D.; Lawler, K. V.; Levchenko, S. V.; Lin, C. Y.; Liu, F.; Livshits, E.; Lochan, R. C.; Luenser, A.; Manohar, P.; Manzer, S. F.; Mao, S.-P.; Mardirossian, N.; Marenich, A. V.; Maurer, S. A.; Mayhall, N. J.; Neuscamman, E.; Oana, C. M.; Olivares-Amaya, R.; O’Neill, D. P.; Parkhill, J. A.; Perrine, T. M.; Peverati, R.; Prociuk, A.; Rehn, D. R.; Rosta, E.; Russ, N. J.; Sharada, S. M.; Sharma, S.; Small, D. W.; Sodt. Advances in molecular quantum chemistry contained in the Q-Chem 4 program package. Mol. Phys. 2015, 113, 184-215.

19. Thomas, A. A.; Speck, K.; Kevlishvili, I.; Lu, Z; Liu, P.; Buchwald, S. L. Mechanistically Guided Design of Ligands That Significantly Improve the Efficiency of CuH-Catalyzed Hydroamination Reactions. J. Am. Chem. Soc. 2017, 140, 13976 13984.

20. Bickelhaupt, F. M.; Houk, K. N. Analyzing Reaction Rates with the Distortion/Interaction-Activation Strain Model. Angew. Chem., Int. Ed. 2017, 56, 10070-10086.

21. Manivasagam, S.; Laury, M. L.; Wilson, A. K. Pseudopotential-Based Correlation Consistent Composite Approach (rp-ccCA) for First- and Second-Row Transition Metal Thermochemistry. J. Phys. Chem. A 2015, 119, 6867-6874.

22. For representative examples where pseudopotentials were used to describe Mo and W, refer to the following: (a) Ishiguro, Y.; Kudo, T.; Muraoka, T.; Ueno, K. Theoretical Study for the Reactions of (Silyl)(silylene)tungsten and molybdenum complexes with Ethylene Sulfide, Organometallics 2014, 33, 2704-2712. (b) Asako, S.; Ishikawa, S.; Takai, K. Synthesis of Linear Allylsilanes vis Molybdenum-Catalyzed Regioselective Hydrosilylation of Allenes. ACS Catal. 2016, 6, 3387-3395, (c) Itabashi, T.; Arashiba, K.; Tanaka, H.; Konomi, A.; Eizawa, A.; Nakajima, K.; Yoshizawa, K.; Nishibayashi, Y. Synthesis and Catalytic Reactivity of Bis(molybdenum-trihalide) Complexes Bridged by Ferrocene Skeleton toward Catalytic Nitrogen Fixation. Organometallics 2019, 38, 2863-2872. (d) Oztopcu, O.; Holzhacker, C.; Puchberger, M.; Weil, M.; Mereiter, K.; 
Veiros, L. F.; Kirchner, K. Synthesis and Characterization of Hydrido Carbonyl Molybdenum and Tungsten PNP Pincer Complexes. Organometallics 2013, 32, 3042-3052. (e) Bouhoute, Y.; Garron, A.; Grekov, D.; Merle, N.; Szeto, K. C.; Mallmann, A. D.; Rosal, I. D. Maron, L., Girard, G.; Gauvin, R. M.; Delevoye, L.; Taoufik, M. Well-Defined Supported Mononuclear Tungsten Oxo Species as Olefin Metathesis Pre-Catalysts. ACS Catal. 2014, 4, 4232-4241. (f) Chen, P.; Zhang, L.; Xue, Z.; Wu, Y.; Zhang, X. Density Functional Theory Study of the Reaction between $\mathrm{d}^{0}$ Tungsten Alkylidyne Complexes and $\mathrm{H}_{2} \mathrm{O}$ : Addition versus Hydrolysis. Inorg. Chem. 2017, 56, 7111-7119. 